\title{
The Siamese-Twin Porphyrin and its Metal Complexes: Platforms for Multi- Electron Redox Chemistry
}

\author{
Dissertation \\ zur Erlangung des mathematisch-naturwissenschaftlichen Doktorgrades \\ "Doctor rerum naturalium" \\ der Georg-August-Universität Göttingen
}

im Promotionsprogramm Chemie

der Georg-August University School of Science (GAUSS)

vorgelegt von

Anastasia Vogel

aus Ekibastus

Göttingen, 2016 



\section{Betreuungsausschuss}

Prof. Dr. Franc Meyer

Institut für Anorganische Chemie, Georg-August-Universität Göttingen

Prof. Dr. Ulf Diederichsen

Institut für Organische und Biomolekulare Chemie, Georg-August-Universität Göttingen

\section{Mitglieder der Prüfungskommission}

Referent:

Prof. Dr. Franc Meyer

Institut für Anorganische Chemie, Georg-August-Universität Göttingen

Korreferent:

Prof. Dr. Ulf Diederichsen

Institut für Organische und Biomolekulare Chemie, Georg-August-Universität Göttingen

Weitere Mitglieder der Prüfungskommission:

Dr. Inke Siewert

Institut für Anorganische Chemie, Georg-August-Universität Göttingen

Jun.-Prof. Dr. Selvan Demir

Institut für Anorganische Chemie, Georg-August-Universität Göttingen

Prof. Dr. Dietmar Stalke

Institut für Anorganische Chemie, Georg-August-Universität Göttingen

Prof. Dr. Thomas Waitz

Institut für Anorganische Chemie, Georg-August-Universität Göttingen

Tag der mündlichen Prüfung: 21. Oktober 2016 

Parts of this thesis have been published in the following journal articles:

A. Vogel, S. Dechert, M. John, C. Brückner, F. Meyer, Chem. Eur. J. 2016, 22, 2307-2316.

A. Vogel, S. Dechert, C. Brückner, F. Meyer, Inorg. Chem. 2017, in press; DOI: 10.1021/acs.inorgchem.6b02916. 



\section{ACKNOWLEDGEMENT}

Дорогие мои родители, мне кажется, что просто »спасибо« не хватит, что бы показать вам то благодарство, которое я чувствую. Спасибо за жизнь в Германии, спасибо за то, что я имею возможность жить, учится и отдыхать так как я хочу, спасибо за всю вашу помощь и терпение. Спасибо за те все времена поддержки и еще больше за те, когда охото было спросить »Wann wirst du fertig?« и не спрашивали.

I would like to thank my supervisor Prof. Dr. Franc Meyer for the opportunity to work on this exciting and challenging topic. I am grateful for your academic support, continuous optimism and encouragement during the past years. Great academic freedom, high scientific standards and excellent laboratory equipment in your group made it possible for me to grow as a scientist and develop a wide set of skills.

Further, I am deeply grateful to Prof. Dr. Christian Brückner for his enormous support and guidance. Your enthusiastic spirit towards chemistry is catching and your attention to detail provided me with new perspectives on my own work. I would like to address my thanks to the examination board and Prof. Ulf Diederichsen for his co-supervision.

I want to express my deepest appreciation to all the people who I met as colleagues and who became my close friends over the last few years - Dr. Mattia Veronelli, Oliver Mitevski, Vandana Goswami, Andreas Schwarz, Dr. Markus Steinert, Dr. Kristian Dalle, Dr. Anne Bretschneider, Dr. Anne Schober. You all have been there, in good times and in bad times, on fun group tours and during devastating experimental results, on memorable evenings and during busy deadline weeks. I value your support, encouragement, advice and consolation.

For a great working atmosphere, I thank my lab-mates Dennis Manz, Pierre Goursot, Tongxun Guo, Ming Li, Pengcheng Duan and especially Andreas Schwarz. Advice and comments by Oliver Mitevski and Alexander Gers-Barlag, as well as the mutual breaks from our laptops in the past months, have been a great help during our shared office time. The members of the group taking care of community tasks such as maintenance of instruments, glove-boxes, distillation of anhydrous solvents, construction of hats, organization of group activities and various purchase responsibilities are gratefully acknowledged for their efforts. I greatly appreciate your time and dedication for the benefit of the community.

This thesis would not have been possible without the help and expertise of so many others: Dr. Serhiy Demeshko, Dr. Sebastian Dechert, Dr. Claudia Stückl, Dr. Michael John, Jörg Teichgräber and continuous support from the analytical departments (NMR spectroscopy, mass spectrometry), the workshops and facility management.

For long-distance emotional support, I thank my friends Ina, Florian, my brother Eugen and especially my partner Daniel. Thank you for your care and encouragement.

I am in debt to Dr. Mattia Veronelli, Dr. Torben Böhnisch, Oliver Mitevski, Sarina Dorazio Ph.D., and Joanne Wong for their meticulous comments during proofreading of this thesis. 



\section{ABBREVIATIONS}

$\varepsilon$

$\lambda$

$\mu$

$\mathrm{acac}^{-}$

$\mathrm{AcO}^{-}$

$\mathrm{Ar}$

$\operatorname{Ar}^{\mathrm{F}}$

$\mathrm{Bu}$

calc.

$\mathrm{Cbl}$

Chl

$\mathrm{CoCp}_{2}$

COSY

CR

CV

cyt

D

DBU

DDQ

DFT

DMSO

eq

EPR

ESI

Et

EtOAc

$\mathrm{FeCp}_{2} / \mathrm{Fc}$

$\mathrm{FeCp}_{2}{ }_{2} / \mathrm{Fc}^{*}$

$\mathrm{Hb}$

HF-EPR

HMBC

HOMO wave number

molar extinction coefficient

wavelength

magnetic moment

acetylacetonate

acetate

aryl

perfluoroaryl

butyl

calculated

cobalamin

chlorophyll

bis( $\left(\eta^{5}\right.$-pentamethylcyclopentadienyl)cobalt(II); decamethylcobaltocene

correlated spectroscopy

charge resonance

cyclic voltammetry

cytochrome

axial zero field splitting parameter

1,8-diazabicyclo[5.4.0]undec-7-en

2,3-dichloro-5,6-dicyano- $p$-benzoquinone

density functional theory

dimethyl sulfoxide

equivalent

electron paramagnetic resonance

electrospray ionization

ethyl

ethylacetate

bis( $\eta^{5}$-cyclopentadienyl)iron(II); ferrocene

bis( $\eta^{5}$-pentamethylcyclopentadienyl)iron(II); decamethylferrocene hemoglobin

high field electron paramagnetic resonance

heteronuclear multiple-bond correlation

highest occupied molecular orbital 


\begin{tabular}{|c|c|}
\hline HR-MS & high resolution mass spectrometry \\
\hline HSQC & heteronuclear single quantum correlation \\
\hline IR & infrared \\
\hline IVCT & intervalence charge transfer \\
\hline $\mathrm{L}$ & ligand \\
\hline LUMO & lowest unoccupied molecular orbital \\
\hline$m / z$ & mass per charge \\
\hline M & metal (ion) \\
\hline$M$ & molecular peak \\
\hline MauG & methylamine utilization protein \\
\hline$M b$ & myoglobin \\
\hline $\mathrm{Me}$ & methyl \\
\hline MS & mass spectrometry \\
\hline $\mathrm{NADP}(\mathrm{H})$ & nicotinamide adenine dinucleotide phosphate \\
\hline NIR & near-infrared \\
\hline NMR & nuclear magnetic resonance \\
\hline NOE & nuclear OvERHAUSER effect \\
\hline OEP & $2,3,7,8,12,13,17,18$-octaethylporphyrin \\
\hline $\mathrm{Ph}$ & phenyl \\
\hline PI & paramagnetic impurity \\
\hline ppm & parts per million \\
\hline py & pyrrole \\
\hline $\mathrm{pz}$ & pyrazole \\
\hline$Q$ & total charge count \\
\hline r.t. & room temperature \\
\hline SOMO & singly occupied molecular orbital \\
\hline SQUID & superconducting quantum interference device \\
\hline TFA & trifluoroacetic acid \\
\hline THF & tetrahydrofuran \\
\hline TIP & temperature-independent paramagnetism \\
\hline TLC & thin layer chromatography \\
\hline TPP & 5,10,15,20-tetraphenylporphyrin \\
\hline $\mathrm{UV} /$ vis & ultraviolet/visible \\
\hline VTVH & variable temperature variable field \\
\hline ZFS & zero field splitting \\
\hline
\end{tabular}




\section{TABLE OF CONTENTS}

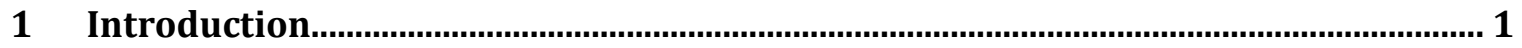

1.1 Porphyrins in Nature 1

1.2 Metal Porphyrins as Models 2

1.3 Expanded Porphyrins and their Aromaticity 4

1.3.1 Aromaticity Concepts 4

$\begin{array}{lll}\text { 1.3.2 Hexaphyrins } & 6\end{array}$

1.3.3 Octaphyrins 8

1.3.4 Metalation of Expanded Porphyrins 9

$\begin{array}{lll}\text { 1.3.5 The Siamese-Twin Porphyrin } & 10\end{array}$

$\begin{array}{ll}\text { 1.3.6 Metalation of the Siamese-Twin Porphyrin } & 12\end{array}$

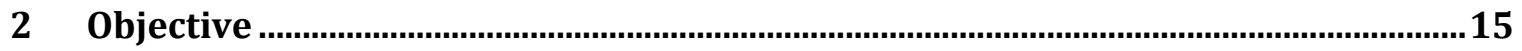

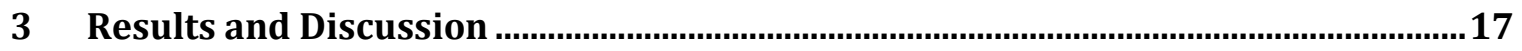

$\begin{array}{lll}3.1 & \text { Oxidation } & 17\end{array}$

3.1.1 Siamese-Twin Porphyrin Oxidation 17

$\begin{array}{lll}\text { 3.1.2 UV/vis Spectral Characterization } & 19\end{array}$

3.1.3 X-ray Crystallographic Molecular Structures 21

$\begin{array}{lll}\text { 3.1.4 NMR Spectroscopic Characterization } & 27\end{array}$

3.1.5 Complexation of Mono-Fused Siamese-Twin Porphyrin 31

$\begin{array}{lll}3.2 & \text { Derivatization } 36\end{array}$

3.2.1 Triazole Building Block 36

$\begin{array}{lll}\text { 3.2.2 Pyrazole Building Block } & 38\end{array}$

3.3 Manganese Complexes $\quad 42$

3.3.1 Siamese-Twin Porphyrin Metalation 42

3.3.2 UV/vis-NIR Spectral Characterization $\quad 45$

3.3.3 X-ray Crystallographic Molecular Structures 48

3.3.4 Magnetic Susceptibility Measurements 52 
3.3.5 Cyclic Voltammetry Measurements $\quad 55$

$\begin{array}{lll}\text { 3.3.6 Photolysis } & 56\end{array}$

3.4 Palladium Complexes $\quad 58$

$\begin{array}{lll}\text { 3.4.1 Siamese-Twin Porphyrin Metalation } & 58\end{array}$

$\begin{array}{lll}\text { 3.4.2 UV/vis Spectral Characterization } & 59\end{array}$

3.4.3 X-ray Crystallographic Molecular Structures 62

3.4.4 NMR Spectroscopic Characterization 66

$\begin{array}{lll}3.4 .5 & \text { Redox Studies } & 70\end{array}$

4 Conclusion

$5 \quad$ Experimental

5.1 Methods $\quad 85$

$\begin{array}{lll}5.2 & \text { Syntheses } & 87\end{array}$

$\begin{array}{lll}\text { 5.2.1 3,5-Dibenzoyl-4-phenyl-1H-pyrazole } 28 & 87\end{array}$

5.2.2 3,5-Bis-(hydroxy(phenyl)methyl-4-phenyl-1H-pyrazole $50 \quad 87$

5.2.3 3,5-Bis-(chloro(phenyl)methyl)-4-phenyl-1H-pyrazole hydrochloride $51 \quad 88$

5.2.4 3,5-Bis-(3,4-diethyl-1H-pyrrole-2-yl-benzyl)-1H-pyrazole 2988

$\begin{array}{lll}\text { 5.2.5 Siamese-Twin Porphyrinogen } 31 \mathrm{H}_{6} & 89\end{array}$

5.2.6 Siamese-Twin Porphyrin $27 \mathrm{H}_{4} \quad 89$

5.2.7 Mono-fused Siamese-Twin Porphyrin $32 \mathrm{H}_{3}$

5.2.8 Bis-fused Siamese-Twin Porphyrin $33 \mathrm{H}_{2} \quad 92$

5.2.9 Nickel Complexes 35HNi and 34Ni 94

5.2.10 Dimanganese Complex 27 $\mathrm{Mn}_{2}{ }^{\text {ac }} \quad 95$

5.2.11 Dimanganese Complex 27 $\mathrm{Mn}_{2}{ }^{\mathrm{az}} 96$

$\begin{array}{lll}\text { 5.2.12 Nickel Manganese Complex 27NiMnac } & 97\end{array}$

$\begin{array}{lll}\text { 5.2.13 Nickel Manganese Complex 27NiMn } & 98\end{array}$

5.2.14 Palladium Complexes $27 \mathrm{H}_{2} \mathrm{Pd}$ and $27 \mathrm{Pd}_{2}$

5.2.15 Monoprotonated Complex 27 $\mathrm{H}_{3} \mathrm{Pd}^{+} \quad 100$

$\begin{array}{lll}\text { 5.2.16 Diprotonated Complex } 27 \mathrm{H}_{4} \mathrm{Pd}^{2+} & 101\end{array}$ 
6 Appendix

6.1 X-ray Crystallographic Details I

6.2 Spectroscopic Details VII

6.3 Computational Details XIX

6.3.1 Optimized Molecular Structures XIX

$\begin{array}{lll}\text { 6.3.2 } & \text { Spin Density Distributions } & \text { XXIV }\end{array}$

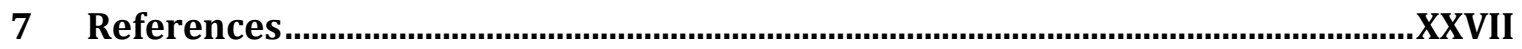

8 Index

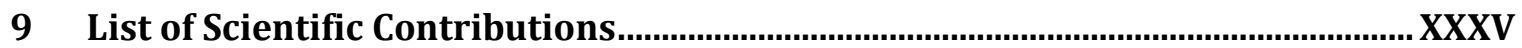

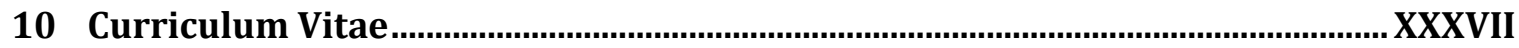





\section{INTRODUCTION}

Tetrapyrrole macrocyclic complexes are the most successful prosthetic groups in metalloproteins. ${ }^{[1]}$ Their presence in every essential life-sustaining process ${ }^{[2,3-5]}$ highlights both their stability by withstanding the test of time and their diversity in adapting to various demands in a myriad of systems. It comes as no surprise that research towards understanding and mimicking of such structures has always been of great interest and encouraged scientists for decades to develop synthetic and functional models of increasing accuracy.

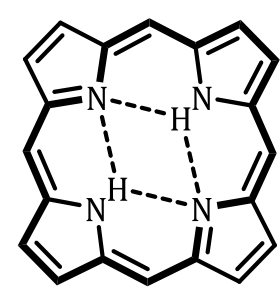

(1a)

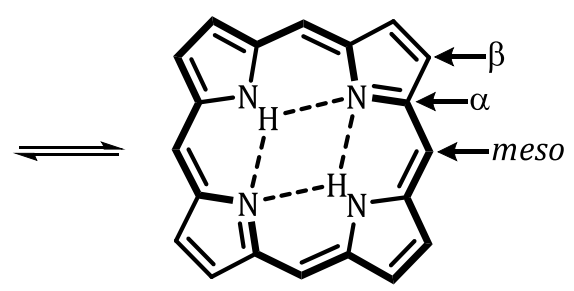

(1b)

Scheme 1: Porphin (1) represents the most elementary tetrapyrrolic macrocycle. $18 \pi$-electron conjugation pathway is highlighted in bold.[6] Simplified nomenclature of substitution positions is indicated by arrows in $\mathbf{1 b}$.

Porphyrins-the prime example for tetrapyrrolic macrocycles-consist of four pyrrole units connected at the $\alpha$-positions through a methine carbon bridge (Scheme 1). Simplified nomenclature according to Scheme 1 will be applied below to refer to peripheral substituents in different positions ( $\beta$ or meso) of the macrocycle.

\subsection{Porphyrins in Nature}

Among the best-studied porphyrinoid complexes in nature are the chlorophylls ( $\mathrm{Chl}$ ), hemoglobins $(\mathrm{Hb})$, myoglobins $(\mathrm{Mb})$, cytochromes $(\mathrm{cyt})$, and cobalamins $(\mathrm{Cbl}) \cdot{ }^{[3]}$ All of these complexes contain a tetrapyrrolic core structure which is specifically modified at the periphery (and/or core) to adapt to the given task and environment. Consequently, the individual roles of the ligand and the central ion in their cooperation may range from basic geometric support to redox reactivity to substrate interactions.

For a more elaborate introduction about the role of porphyrinoid complexes in nature, cytochrome P450 is chosen as an example. The cytochrome P450 superfamily is one of the most relevant in the group of hemoproteins and includes diverse enzyme variations; in humans, they are mainly located in the liver. All of these enzymes are known for their detoxifying function in the body carrying out approximately $75 \%$ of human drug metabolism. ${ }^{[3,4,7]}$

\section{Cytochrome P450}

All cytochromes are membrane-bound, redox-active hemoproteins and can be classified according to their spectroscopic and structural features, as well as reduction potentials or inhibitor sensitivity. ${ }^{[3]}$ The reduced and $\mathrm{CO}$ binding form with its major spectroscopic feature at $\lambda_{\max }=450 \mathrm{~nm}$ is eponymous for this particular family of enzymes. ${ }^{[8]}$ 


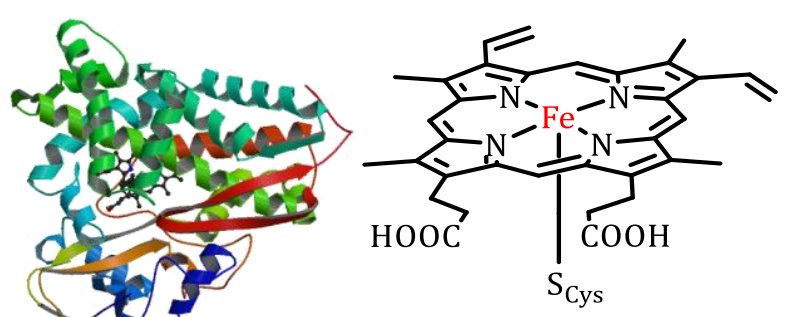

(2)

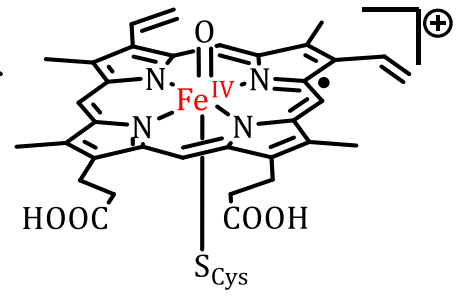

(3)

Figure 1: Ribbon representation of bacterial camphor induced $\mathrm{P} 450_{\text {cam }}$ (left), its heme $b$ cofactor (2, middle), and a proposed key intermediate, so-called Compound I (3, right), of the catalytic cycle during monooxygenase activity.[5,9]

The enzymatic reactivity of cytochrome P450 (Figure 1) is found in nearly all organisms.[10] Its primary biological task is monooxygenase activity, transferring one oxygen atom from $\mathrm{O}_{2}$ onto a variety of apolar substrates, with the remaining oxygen atom converted into a water molecule (Equation (1)).[3] Compound I (Figure 1,3) represents one of the key intermediates believed to form during the catalytic cycle.[11] This intermediate is postulated to be an oxoFe(IV) species with an additional oxidizing equivalent delocalized over the porphyrin (as a $\pi$-radical cation) and thiolate ligands. The reduction equivalents for completeness of the catalytic cycle are provided by NADH/NADPH, flavins and flavoproteins via reductases.

$$
\mathrm{R}-\mathrm{H}+\mathrm{O}_{2}+2 \mathrm{e}^{-}+2 \mathrm{H}^{+} \stackrel{P 450}{\longrightarrow} \mathrm{R}-\mathrm{OH}+\mathrm{H}_{2} \mathrm{O}
$$

The ability to coordinate a substrate is mirrored in the structure of the active center. Here a heme $b$ cofactor (Figure 1, 2) is located in the enzyme pocket and its central iron ion is coordinated by an additional anionic cysteine residue in one axial position. Aside from tethering the active center to the protein, the thiolate ligand also plays a crucial role in the enzymatic activity through stabilizing high oxidation states of the metal ion and conferring high basicity on the oxo-unit in Compound I. The opposite axial position is occupied by a weakly bound water molecule in the resting state. Throughout the catalytic cycle the porphyrin system participates in the redox chemistry, emphasizing its redox noninnocence. ${ }^{[3,4,7,12]}$ The full catalytic cycle is considered a dynamic system with not necessarily linear progress and its detailed intermediates are still under debate (cf. Chapter 1.2).[7,9]

\subsection{Metal Porphyrins as Models}

To facilitate and promote a discussion on possible intermediates and reactions in the catalytic cycle of cytochrome P450, a variety of spectroscopic methods has been applied.[13] Additionally, synthesis and characterization of model complexes provided valuable insights into structural details and reactivity pathways.[14,15] Herein, the vast majority of today's understanding of P450 structure-function relationships is based on the P450 ${ }_{\text {cam }}$ models. ${ }^{[16]}$

Functional model systems primarily contain iron and manganese, and at times chromium and ruthenium, porphyrin complexes.[12,14,17] Pioneering works of HILL, Groves, ChANG and MEUNIER in the 1980s established the first milestones of synthetic functional models.[18] Shortly thereafter, outstanding work published by SLIGAR reporting on a manganesesubstituted $\mathrm{P} 450_{\text {cam }}\left(\mathrm{Mn}-\mathrm{P} 450_{\mathrm{cam}}\right)$ helped to define the crucial role assigned to the axially bound thiolate ligand in the enzyme pocket.[19] 

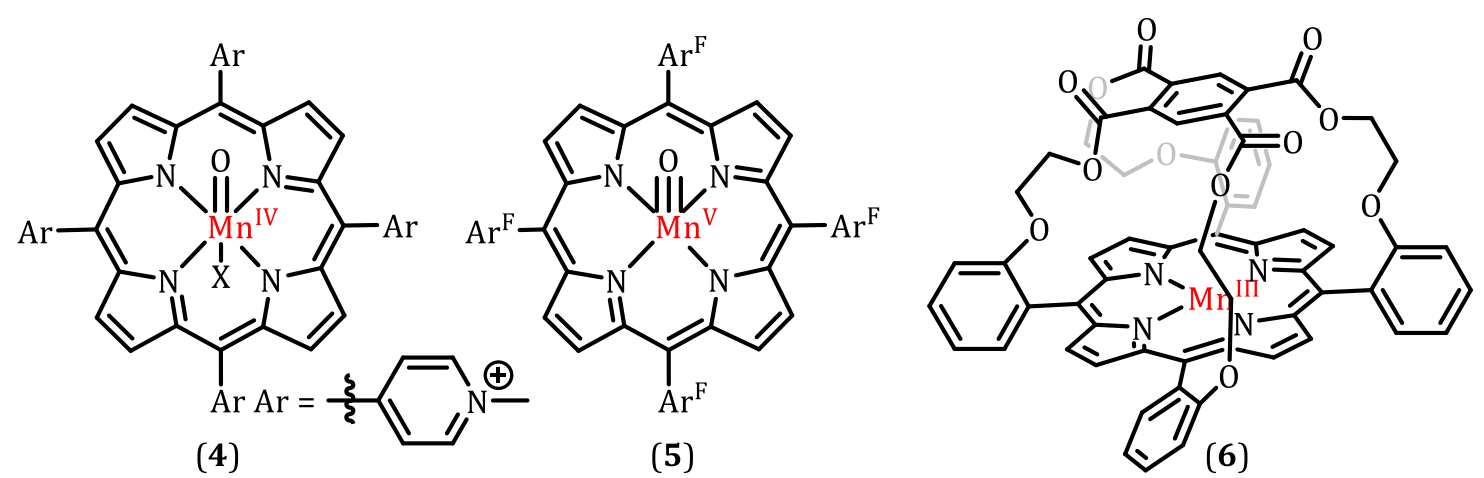

Figure 2: High-valent oxomanganese complexes 4 (left) and 5 (middle) and a capped TPP derivate $\mathbf{6}$ (right).[12,20]

To efficiently mimic enzyme reactivity, readily available meso-substituted porphyrin manganese complexes such as $\mathbf{4}$ and $\mathbf{5}$ (Figure 2) surprisingly proved superior to more sophisticated substitution patterns such as the capped complex 6 (Figure 2) and related systems containing iron. ${ }^{[12,20]}$ Analyses in gas phase and solution repeatedly confirmed the formation of both high-valent oxo-Mn(IV) and oxo-Mn(V) species (Figure 2, 4 and 5), analogous to intermediates proposed for the catalytic cycle of P450.[21] Moreover, the electronic interaction between the metal ion and the ligand, as the proposed $\pi$-cation formation in Compound I (Figure 1, 3), is highly debated in the literature and the supportive redox non-innocence of the porphyrinic macrocycle received great attention. ${ }^{[12,22]}$

The results of such studies, however, are highly dependent on the geometry and electronic topology of the model complex and the nature of the substrate. Thus, no final agreement has been reached yet and a reconciliation of the hitherto existing studies outlined above must admit the possibility of multiple catalytic pathways.[12,17]

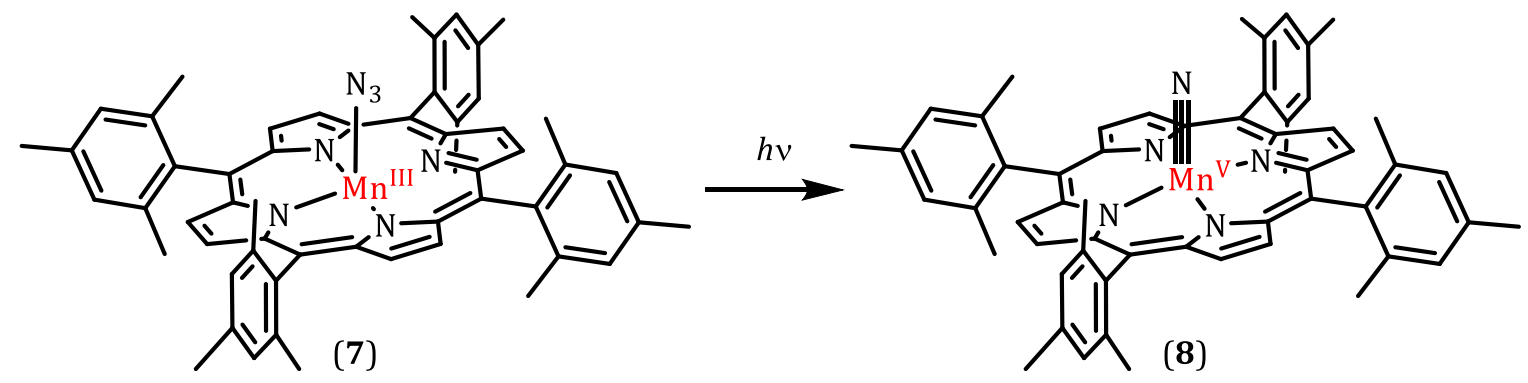

Scheme 2: Irradiation of an azido manganese(III) precursor (7) results in the formation of a high-valent, catalytically active $\{\mathrm{Mn}(\mathrm{v}) \mathrm{N}\}$ species (8).[23]

The vibrant discussion on oxo-metal porphyrin complexes has further inspired research towards high-valent imido and nitrido complexes $(\mathbf{8}$, Scheme 2$)$ with analogous reactivity of oxidative amination. ${ }^{[23-26]}$ Herein, manganese azido precursors (7) proved particularly successful. Irradiation (Scheme 2) of such readily available complexes resulted in the formation of $\{\mathrm{Mn}(\mathrm{V}) \mathrm{N}\}$ species ${ }^{[27]}$ which showed catalytic activity towards unactivated hydrocarbons. ${ }^{[26]}$ Remarkably, even though $\mathrm{Mn} \equiv \mathrm{N}$ is isoelectronic to the highly reactive $\mathrm{Fe}=0$ unit, nitride metalloporphyrins were shown to be thermally and chemically stable.[24]

While all these examples describe catalytic conversions on a milli- or microgram scale and focus on methodology, more recent attempts devote their attention to the development of more robust catalysts applied in scaled-up transformations. ${ }^{[28]}$ 


\subsection{Expanded Porphyrins and their Aromaticity}

The importance of porphyrinoid subunits in nature and in model systems led to an enormous interest in the study of their artificial analogs. Since its beginning in the 1960s, porphyrin research led to a series of variations in the backbone substitution, modifications of the core and introduction of several heteroatoms. After a labored start in the 1960s and 1970s, the study of porphyrinoid systems accelerated dramatically due to its newly discovered potential for anion recognition, photodynamic therapy, functional dyes, aromaticity, and magnetic resonance imaging.[6] Going beyond nature, the flourishing synthetic prospects resulted in many adjacent research fields - including but not limited to expanded, contracted, coremodified, assembled, inverted or confused porphyrins (Figure 3).[29-32,33]

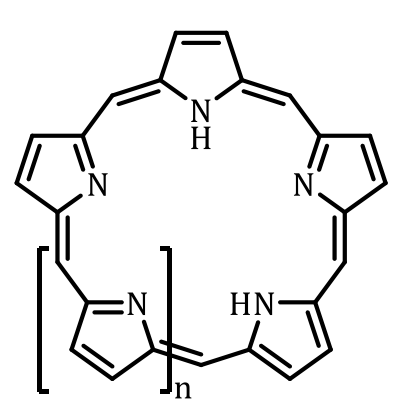

(9) $\mathrm{n}=1,2,3 \ldots$

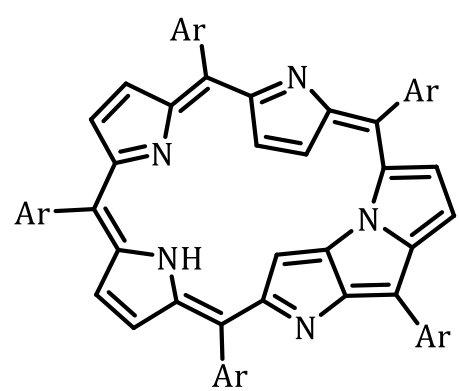

(11)

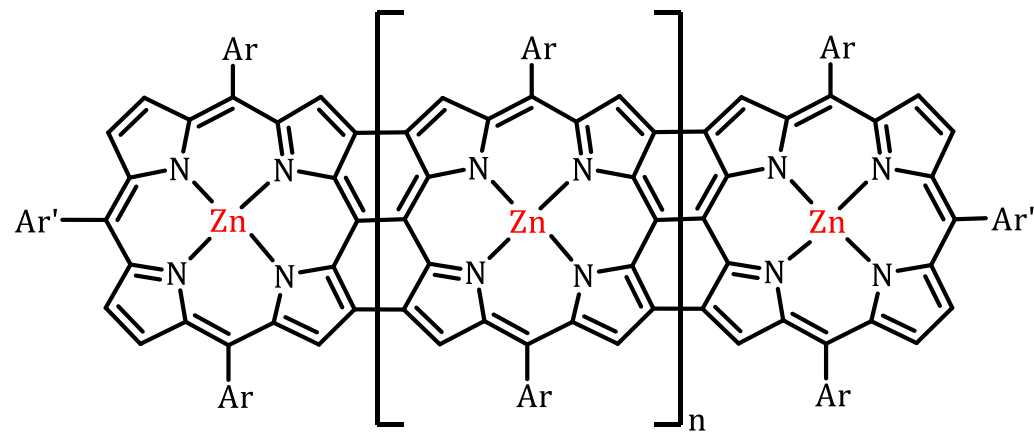

(10)

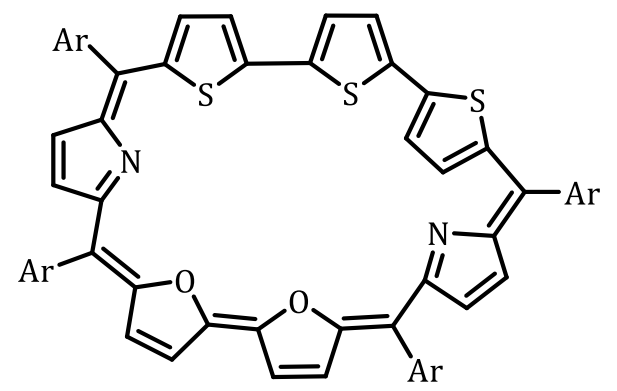

(12)

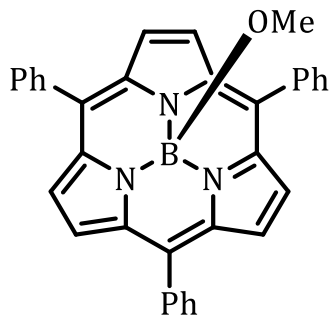

(13)

Figure 3: Selected research fields in porphyrin chemistry: expanded porphyrins (9), porphyrin arrays (10), $\mathrm{N}$-fused and inverted porphyrins (11), core-modified porphyrins (12), and subporphyrins (13).[29,31,34,35]

Expanded[6,29,31] and modified ${ }^{[36]}$ porphyrins are intriguing study objects because of their redox-, temperature-, solvent-, $\mathrm{pH}-$, and substituent-dependent physical properties. Due to the sheer number of different topics associated with expanded and modified porphyrins, not all aspects can be covered in the following chapters. However, there are excellent overview articles and reviews on all major issues - from a general summary ${ }^{[6]}$ to more specific topics such as synthetic strategies,[29] core modifications,[32] introduction of new building blocks, ${ }^{[36,37]}$ aromaticity, ${ }^{[30,38]}$ and metalation. ${ }^{[39]}$ Aspects most relevant to this work will be elaborated in more detail below.

\subsubsection{Aromaticity Concepts}

In comparison to 'common' porphyrins that express their flexibility via doming, saddleshaping or ruffling of the tetrapyrrolic backbone,[3] expanded porphyrins gain further conformational flexibility with each additional pyrrolic subunit. Of particular interest is then 
the expression of reversible topological switches. Each spatial rearrangement in the oligopyrrolic backbone is accompanied by changes in the aromatic properties of the macrocycle.[30,40-42]

Hereby the classic concept of aromaticity according to HücKEL has to be extended by the concept of MöBIUS aromaticity (Table 1).[6,43-45] Classic HÜCKEL aromatic compounds carry [4n+2] $\pi$-electrons in a planar, circularly arranged, conjugated system and show increased stability due to electron delocalization. Accordingly, [4n] $\pi$-electron-bearing systems are considered HÜCKEL antiaromatic and less stabilized.[43] Conversely, idealized MöBIUS aromatic compounds carry [4n] $\pi$-electrons in a monocyclic array of molecular orbitals in which there is an odd number of out-of-phase overlaps. $A[4 n+2] \pi$-electron count here is indicative of an antiaromatic species.[43] In general, MöBIUS-type expanded porphyrins are not expressed spontaneously, but rather require support by for instance protonation or metalation.

Table 1: Commonly found topologies and orbital arrangements in expanded porphyrins. ${ }^{[6,45,46]}$

\begin{tabular}{c|c|c|c}
$\begin{array}{c}\text { Hückel } \\
\text { (planar) }\end{array}$ & $\begin{array}{c}\text { Hückel } \\
\text { (figure-eight) }\end{array}$ & $\begin{array}{c}\text { Möbius } \\
\text { (singly twisted) }\end{array}$ \\
\hline aromaticity rules & $\begin{array}{c}\text { [4n+2]: aromatic } \\
\text { [4n]: antiaromatic }\end{array}$ & $\begin{array}{c}\text { non-aromatic } \\
\text { or } \\
\text { [4n+2]: weakly aromatic } \\
\text { [4n]: weakly antiaromatic }\end{array}$ & $\begin{array}{c}\text { [4n]: aromatic } \\
{[4]}\end{array}$ \\
\hline
\end{tabular}

Aside from these clear-cut cases, there are a number of topologies showing partial compliance to the strict rules. An important case is the figure-eight topology (Table 1, center):[43] weak HÜCKEL aromaticity (for [ $4 n+2] \pi$-electrons) may be retained even though planarity is lifted when the macrocycle undergoes a double twist. ${ }^{[42,43,45]}$ However, numerous cases of geometrically unrestricted expanded porphyrins adopting figure-eight topologies are classified as non-aromatic. Only when metalation, protonation or skeletal fusions enforce

Table 2: Characteristic spectroscopic features of aromatic and antiaromatic systems.[6]

\begin{tabular}{c|c|c} 
& aromatic & antiaromatic \\
\hline HOMO-LUMO gap & large & small \\
UV/vis features & $\begin{array}{c}\text { sharp SoRET-band } \\
\text { distinct Q-like bands }\end{array}$ & $\begin{array}{c}\text { broad and poorly defined } \\
\text { no Q-like bands }\end{array}$ \\
excited-state lifetimes & long & short \\
NMR features & {$[4 \mathrm{n}+2]:$ low $\delta$-values } \\
(inner protons) & {$[4 \mathrm{n}]$ : high $\delta$-values }
\end{tabular}


rearrangements and lock the macrocycles in a distinct desired geometry, either aromaticity case may be favored.[30,38]

Each given case of aromaticity can be evaluated by several criteria:[6,47] especially geometric considerations on the basis of X-ray crystallographic data, ${ }^{1} \mathrm{H}$ NMR chemical shifts, characteristic features in UV/vis absorption spectra and theoretical calculations (Table 2). However, the boundaries between the respective classes remain blurred as larger macrocycles tend to be flexible or engage in fluent equilibria. It is to be pointed out that the different topologies are not simply an academic exercise but may be applied, particularly due to their unique spectroscopic features, for instance in chiral sensing.[48]

\subsubsection{Hexaphyrins}

The most extensively studied expanded porphyrin is meso-perfluoroaryl-substituted hexaphyrin $\left(\mathbf{1 4 H}_{2}\right.$, Scheme 3).[51] Its success is partially attributed to a simple one-pot synthesis with satisfactory yields[34] and partially to the decade-long pursuit to understand its intriguing structural and electronic nature. ${ }^{[49]}$ Against the background of the extended aromaticity concepts presented above, the oxidized form $\left(\mathbf{1 4 H}_{\mathbf{2}}\right.$, Scheme 3) of [26] hexaphyrin(1.1.1.1.1.1) adopts a rectangular and almost planar conformation with two inverted pyrroles on the long sides, showing strong HüCKEL aromaticity.[52] An enhancement of the aromatic character can be induced by deprotonation. ${ }^{[53]}$ Conversely, this aromaticity is retained but weakened when the macrocycle is forced into a figure-eight conformation by

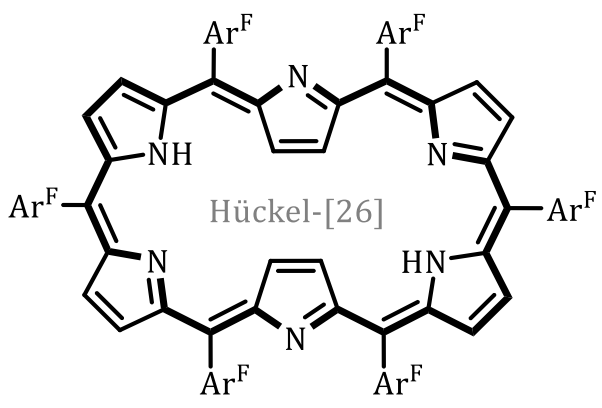

$\left(14 \mathrm{H}_{2}\right)$

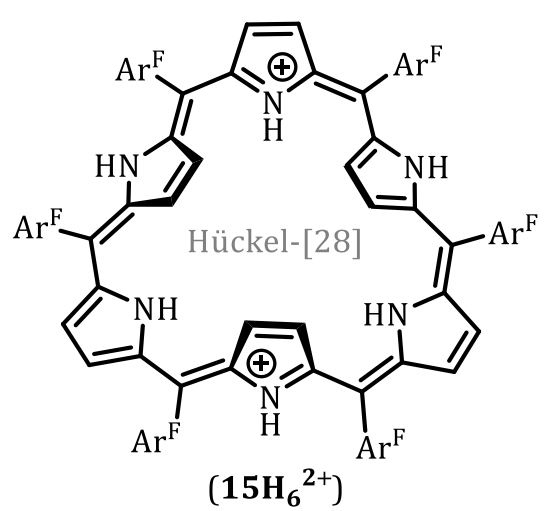

$+2 \mathrm{e}^{-}$

$\stackrel{+2 \mathrm{H}^{+}}{\longrightarrow}$

Ar

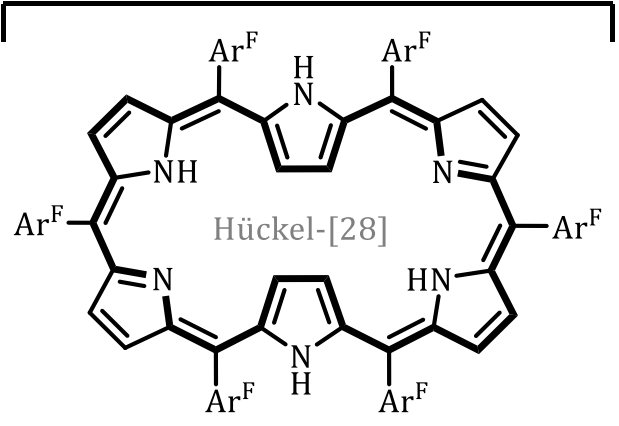

$\left(15 \mathrm{aH}_{4}\right)$
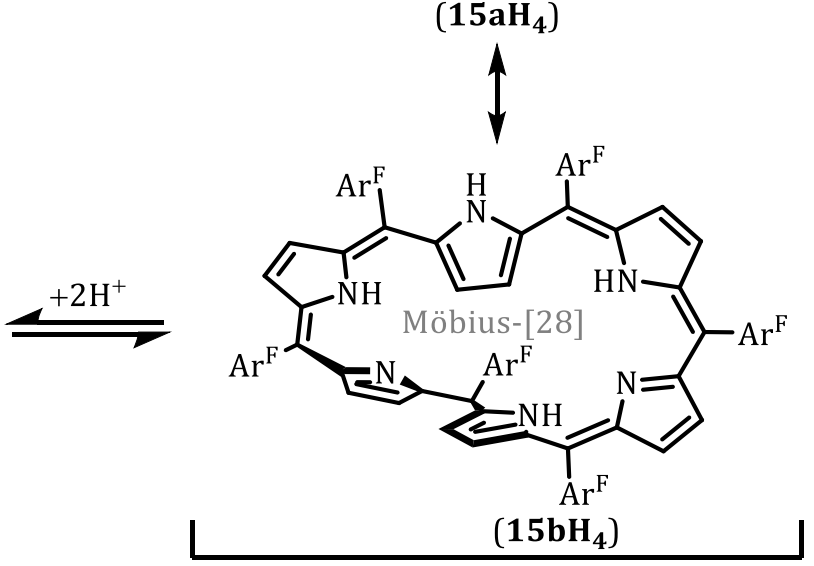

Scheme 3: [26]Hexaphyrin(1.1.1.1.1.1) $\left(\mathbf{1 4 H}_{\mathbf{2}}\right)$, its two-electron reduction product [28] hexaphyrin in HüCKEL antiaromatic $\left(\mathbf{1 5 a H}_{4}\right)$ and Möbius aromatic $\left(\mathbf{1 5 b H}_{4}\right)$ conformations, and a diprotonated HücKEL antiaromatic triangular [28] hexaphyrin $\left(\mathbf{1 5 H}_{\mathbf{6}}{ }^{\mathbf{2}}\right) \cdot[49,50]$ Conjugation pathways for $\mathbf{1 4 H}_{\mathbf{2}}$ and $\mathbf{1 5 a \mathbf { H } _ { 4 }}$ are highlighted in bold. 
introduction of bulkier substituents at the $\beta$-positions.[50,54] Reduction of the [26]hexaphyrin (Scheme 3) yields the [28] hexaphyrin in a dynamic mixture of rectangular HücKEL antiaromatic $\left(\mathbf{1 5 a H}_{\mathbf{4}}\right)$ and singly twisted MöBIUS aromatic $\left(\mathbf{1 5} \mathbf{b H}_{\mathbf{4}}\right)$ conformations.[49,54] Further topological changes occur upon mono-and diprotonation of the macrocycles (e.g. $\left.{ }_{15} \mathbf{H}_{6}{ }^{2+}\right) .[50]$

While protonation and temperature control offer a mild approach to reversibly switch the topology of hexaphyrins, harsher oxidative conditions (higher temperatures or longer reaction times) lead to irreversibly fused products (Figure $4,16-19$ ) in which, for example, the ortho-fluoro groups of the perfluorinated aryl substituents undergo nucleophilic aromatic substitution reactions. The nucleophile in these reactions is either a nitrogen atom of an inverted pyrrole moiety (as in $\mathbf{1 6}$ and 19) or a $\beta$-oxygen introduced by an aerial oxidation reaction (as in 17).[55,56] In addition, meso-3-thienyl groups have been shown to be susceptible to oxidative fusions to inverted pyrrole nitrogen atoms (16).[57] Such fused products are generally more restricted in their conformational flexibility, thus allowing direct realization of MöBIUS aromatic conformations without further assistance of metal coordination, protonation or temperature control.[6]

Aside from peripheral fusion reaction, transannulation upon heating is a common pathway for expanded porphyrins to escape steric tension. ${ }^{[58]}$ The vinylene-bridged product (Figure 4, 18) shows a conformationally highly restricted structure and thus exhibits a facile redoxinterconversion between HÜCKEL aromatic and antiaromatic states.[59]

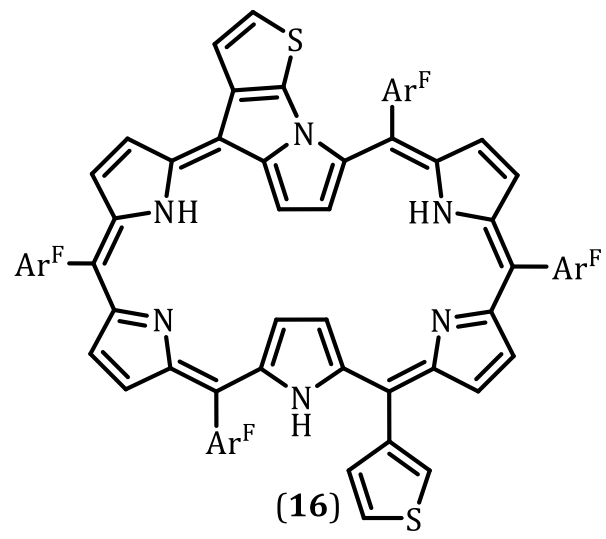

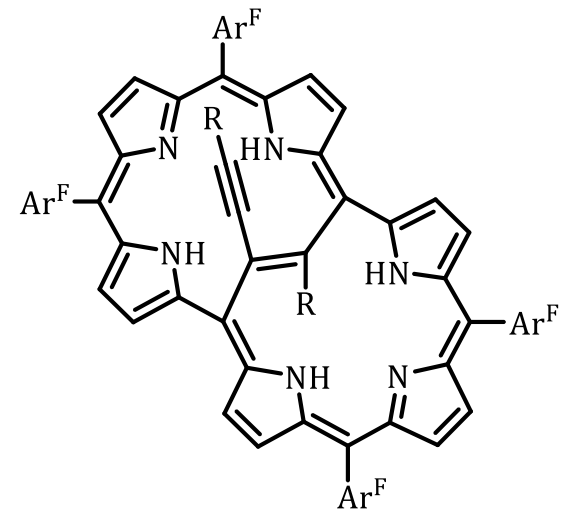

(18)

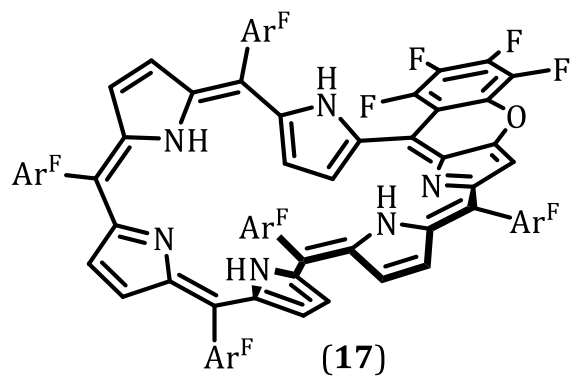

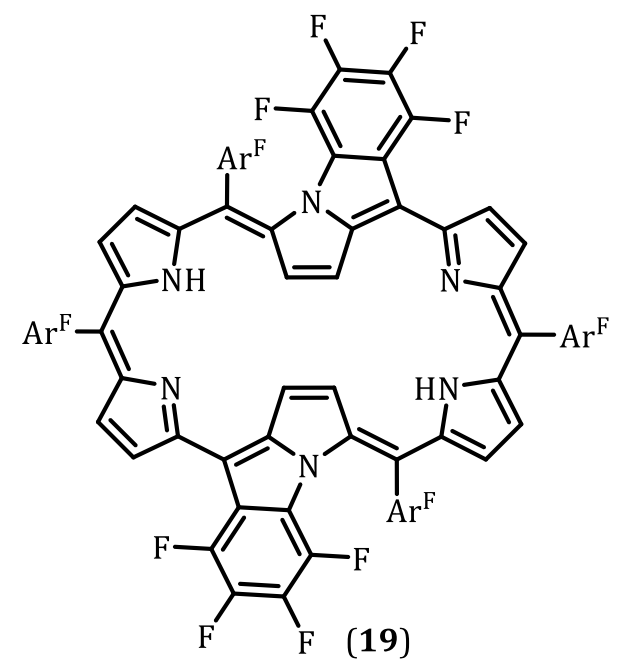

Figure 4: Examples of fused expanded porphyrins: singly thienyl-fused [28]hexaphyrin (16), benzopyrane-fused [28]hexaphyrin (17), ethylene-bridged [28]hexaphyrin (18), and doubly N-fused [26]hexaphyrin (19).[55-58] 


\subsubsection{Octaphyrins}

Octaphyrins represent the second major frequently reported class of expanded porphyrins.[60-63] They appear in a number of different variations in meso-linkages and peripheral substitution patterns. Also here, ethyl substitution in $\beta$-positions enforces a figureeight conformation of the highly flexible backbone with all pyrrolic nitrogen atoms pointing inwards engaged in intramolecular hydrogen bonding $\left(\mathbf{2 0 H}_{\mathbf{4}}\right.$, Figure 5).[60] Thus, parent octaphyrins are inherently non-aromatic in their free-base form independent of the theoretical electron count.

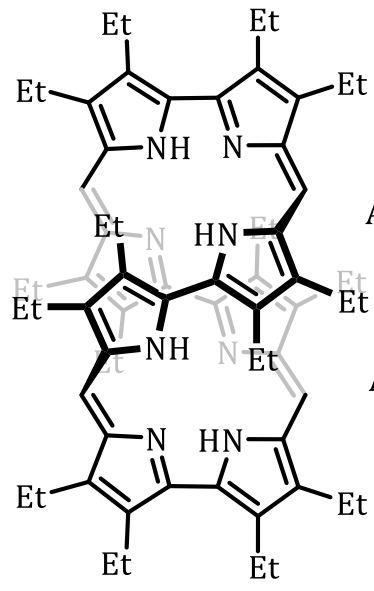

$\left(2 \mathrm{OH}_{4}\right)$
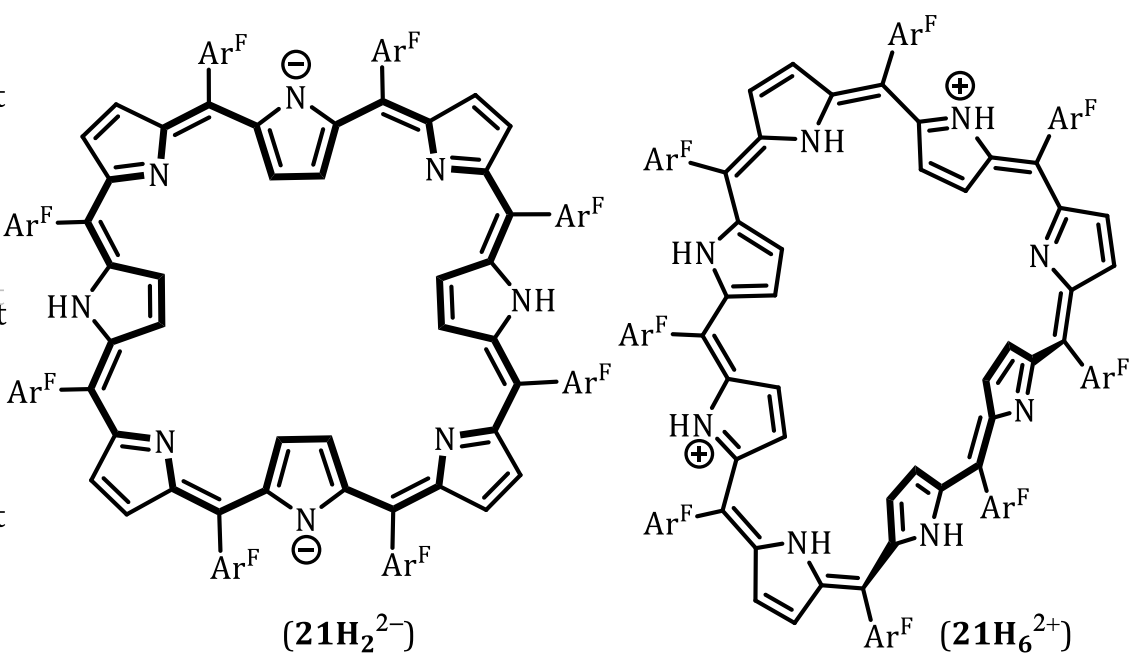

Figure 5: Figure-eight shaped, $\beta$-ethyl-substituted and non-aromatic [32] octaphyrin(1.0.1.0.1.0.1.0) (20H4), meso-perfluoroaryl-substituted [36]octaphyrin(1.1.1.1.1.1.1.1) as a dianionic, rectangular HüCKEL antiaromatic species $\left(\mathbf{2 1 H}_{2}{ }^{2-}\right)$ and diprotonated, twisted MöBıUS aromatic species $\left(\mathbf{2 1 H}_{\mathbf{6}}{ }^{\mathbf{2}}\right) .{ }^{[60-62]}$ Conjugation pathway for $\mathbf{2 1 H}_{\mathbf{2}^{2-}}$ is highlighted in bold.

The non-aromatic octaphyrin $\mathbf{2} \mathbf{0 H}_{\mathbf{4}}$ (Figure 5) as well as a series of corresponding metal complexes $\mathbf{2 0 M}^{1} \mathbf{M}^{2}$ (Figure 6) were analyzed in remarkable detail towards their electrochemical properties.[64] The study revealed the non-innocent behavior of the macrocycle and significant spectroscopic changes upon two-fold electrochemical reduction. However, the authors solely conclude to rule out HüCKEL aromaticity due to the twisted structure.[64] From today's broadened understanding of aromaticity, it might be reasonably assumed that the generated species complies with MöBIUS rule for antiaromaticity.[65]

In contrast to $\beta$-ethyl substitution, perfluoroaryl substitution in meso-positions provides the extended macrocycle with higher rotational flexibility at the pyrrolic subunits (Figure 5, $\mathbf{2 1 H}_{2} \mathbf{2}^{\mathbf{2}}$ and $\mathbf{2 1} \mathbf{H}_{6}{ }^{\mathbf{2}}$ ).[61,66] Thereby, $\mathrm{pH}$-dependent conformational rearrangements are facilitated and (anti-)aromatic topologies are expressed. Doubly twisted figure-eight conformation of neutral meso-perfluoroaryl-substituted [36]octaphyrin(1.1.1.1.1.1.1.1) is dramatically changed upon deprotonation to yield the dianionic, rectangular HüCKEL antiaromatic species $\left(\mathbf{2 1 H}_{2}{ }^{2-}\right) \cdot{ }^{[61]}$ In contrast, two-fold protonation leads to an anion-supported, twisted MöBIUS aromatic topology $\left(\mathbf{2 1 H}_{\mathbf{6}}{ }^{\mathbf{}}\right)$. [62] Both rearrangements are enabled by pyrrole inversion of the charge-carrying units and supported by electrostatic, intermolecular interactions with counterions and solvent molecules.[61,62] 


\subsubsection{Metalation of Expanded Porphyrins}

Expanded porphyrins form a wide range of metal complexes (Figure 6, 20M $\mathbf{M}^{\mathbf{1}} \mathbf{M}^{2}$ ) and adopt a variety of coordination motifs enforcing significant geometric rearrangements within the backbone of the entire macrocycle.[6] Different coordination modes (e.g. 23Pd ${ }_{2} \mathbf{C u}$ in Figure 6; $\mathrm{Cu}(\mathrm{II})$ in a distorted square-planar $\left\{\mathrm{N}_{4}\right\}$-coordination, $\mathrm{Pd}(\mathrm{II})$ in square-planar $\left\{\mathrm{N}_{2} \mathrm{C}_{2}\right\}$ - and Tshaped $\left\{\mathrm{N}_{2} \mathrm{C}\right\}$-coordination with an additional agostic interaction) and multiple metal ion coordination (Figure 6, 20M $\mathbf{M}^{1} \mathbf{M}^{2}$ and ${ }^{23 P d_{2}} \mathbf{C u}$ ) allow the formation of unprecedented complexes with intriguing structural, electronic, and magnetic properties as well as novel reactivity (Figure 6, 24Ni $\mathbf{N i}_{2}$ ). $\left.64,67,68\right]$

Numerous examples of late transition metal complexes have been reported,[39] while examples of early transition metal complexes remain scarce.[69] In particular, $\mathrm{Co}(\mathrm{II})-, \mathrm{Ni}(\mathrm{II})-$,

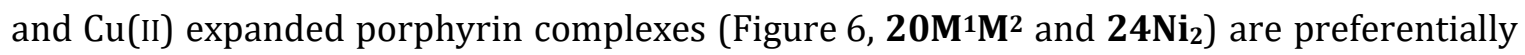
formed.[64,70] Despite readily accessible redox potentials of these metal ions, cyclovoltammetric analyses often show ligand centered redox reactions and even formation of stable, aromatic $\pi$-cations. $[64,68,71]$
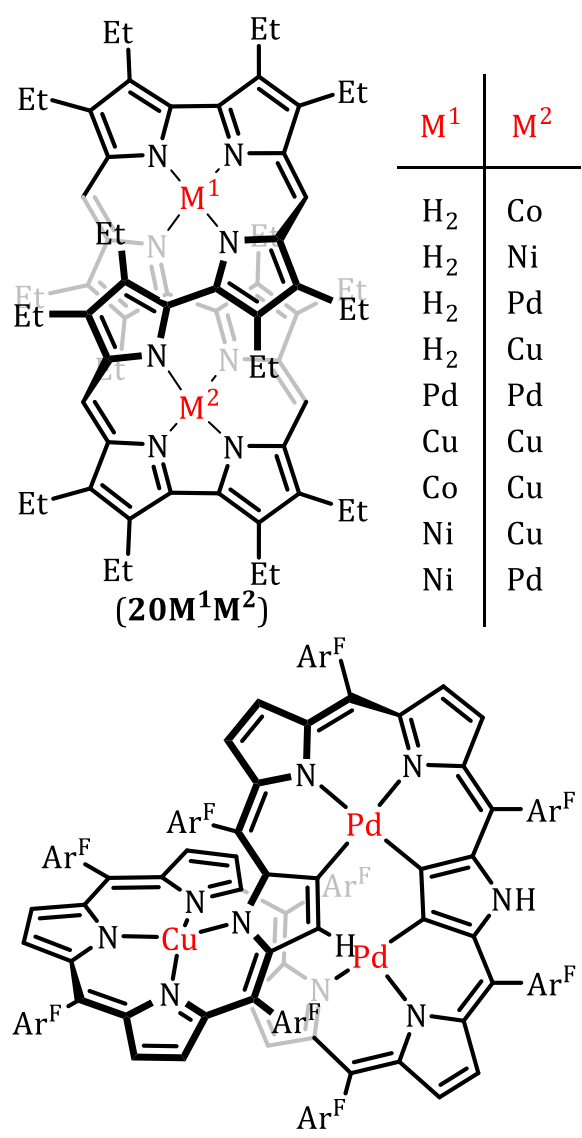

$\left(23 \mathrm{Pd}_{2} \mathrm{Cu}\right)$

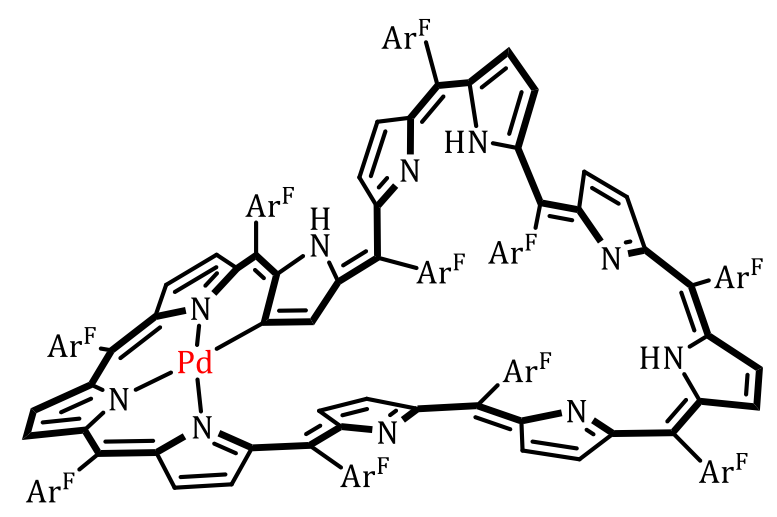

$\left(22 \mathrm{H}_{3} \mathrm{Pd}\right)$

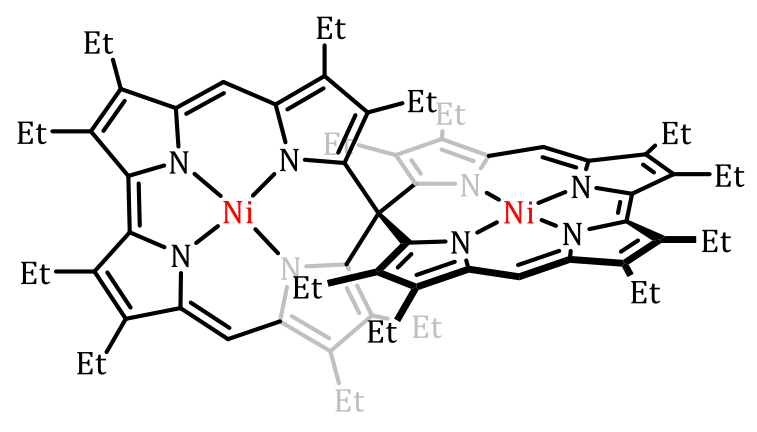

$\left(24 \mathrm{Ni}_{2}\right)$

Figure 6: Selection of (multi-)metalated expanded porphyrins: numerous homo- and bimetallic $\beta$-ethylsubstituted non-aromatic [32] octaphyrin(1.0.1.0.1.0.1.0) complexes (20M11 $\left.\mathbf{M}^{2}\right)$, meso-perfluoroaryl-substituted [44] decaphyrin(1.1.1.1.1.1.1.1.1) palladium complex $\left(\mathbf{2 2} \mathbf{H}_{3} \mathbf{P d}\right)$ representing, to date, the largest MöBIUS aromatic metalated macrocycle, trismetalated [40]nonaphyrin(1.1.1.1.1.1.1.1.1) (23Pd2Cu), and a spirodiporphyrin nickel complex (24Ni $)$.[64,67,72,73] 
Furthermore, palladium is commonly used to induce geometric rearrangements and subsequently enforce aromaticity switching in large and inherently flexible expanded porphyrins (Figure 6). [72] The diamagnetic nature of such complexes provides an additional opportunity to employ NMR spectroscopic techniques for detailed comparative insights into topology and aromaticity of the parent macrocycle and its corresponding metal complex.

A remarkable example in this regard is the [44]decaphyrin(1.1.1.1.1.1.1.1.1.1) (Figure 6, $\mathbf{2 2}_{3} \mathbf{P d}$ ). In its free base form, the macrocycle adopts a crescent shape and exhibits a ${ }^{1} \mathrm{H}$ NMR signal pattern consistent with a non-aromatic expanded porphyrin. ${ }^{\text {[2] }}$ Reductive palladium metalation leads to the formation of three distinctly new topologies: an untwisted HüCKEL aromatic [46]decaphyrin complex, a doubly twisted (figure-eight) HÜCKEL antiaromatic [44]decaphyrin complex upon reoxidation and a singly twisted MöBIUS aromatic [44] decaphyrin complex $\mathbf{2 2} \mathbf{H}_{3} \mathbf{P d}$ (Figure 6) upon further isomerisation. ${ }^{\text {[2] }}$ These complexes represent the largest HÜCKEL aromatic, HÜCKEL antiaromatic and MÖBIUS aromatic molecules locked by coordination of a $\mathrm{Pd}(\mathrm{II})$ ion to date. The classification of the respective aromatic character of each has been confirmed on the basis of X-ray molecular structures, calculations of diatropic and paratropic ring currents, absorption characteristics, and excited-state dynamics. ${ }^{[72]}$

Another exceptional expanded porphyrin complex is formed in an unexpected cascade of reactions occurring upon treatment of hexadecaethyl octaphyrin(1.1.1.0.1.1.1.0) with nickel(II) acetate in refluxing DMF. ${ }^{[73,74]}$ The resulting spirodiporphyrin nickel complex (24Ni 2 , Figure 6) shows significant reciprocal influence of both orthogonally arranged conjugated systems onto each other. This so-called spiroconjugation results in measurable effects on the overall molecular properties like oxidation potentials and UV/vis spectroscopic features.[73]

\subsubsection{The Siamese-Twin Porphyrin}

A unique and ingenious expanded porphyrin is represented by the Siamese-twin porphyrin $\mathbf{2 7 H}_{\mathbf{4}}$ (Scheme 4). This macrocycle was proposed by LIND in 1987 and synthesized by BLUSCH* in 2011.[75-77] This unprecedented molecule is characterized by the presence of two porphyrin-like binding pockets conjoined by two pyrazole moieties. Figuratively, it represents a merge of the well-known 2,3,7,8,12,13,17,18-octaethylporphyrin (OEP, $\mathbf{2 5} \mathbf{H}_{2}$ ) and 5,10,15,20-tetraphenylporphyrin (TPP, $\mathbf{2 6 \mathbf { H } _ { 2 }}$ ).

The macrocyclic size and connectivity resemble closely hexaphyrins, while its electronic structure differs highly from aromaticity found in the all-pyrrolic hexamers: the cohesive pyrazole unit-though accomplishing the joint-interrupts the overall macrocyclic conjugation. Each pocket forms an isolated conjugation pathway bearing $14 \pi$-electrons. Consequently, Siamese-twin porphyrin $\mathbf{2 7} \mathbf{H}_{4}$ does not possess macrocycle aromaticity.[76]

${ }^{*}$ née FRENSCH 


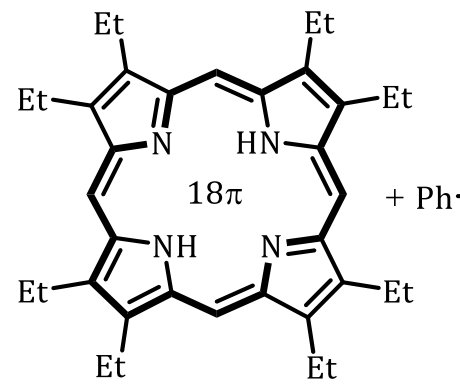

$\left(25 \mathrm{H}_{2}\right)$

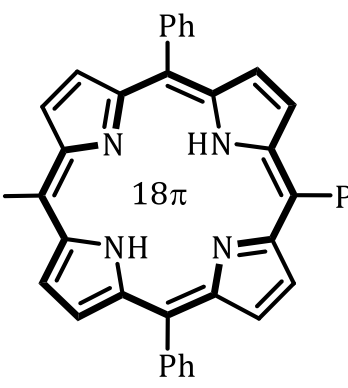

$\left(26 \mathrm{H}_{2}\right)$

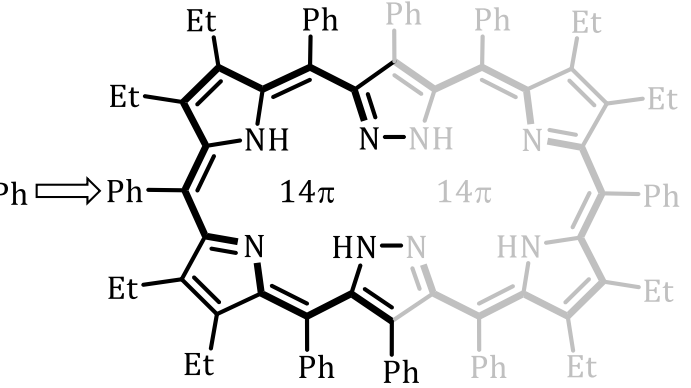

$\left(27 \mathbf{H}_{4}\right)$

Scheme 4: Figurative merging of OEP $\left(25 \mathrm{H}_{2}\right)$ and TPP $\left(26 \mathrm{H}_{2}\right)$ to yield the conjoined backbone and advantageous substitution pattern of the Siamese-twin porphyrin $\left(\mathbf{2 7 H}_{\mathbf{4}}\right) .{ }^{[76]}$ Conjugation pathways are highlighted in bold. The two distinct hemicycles are colored in black and grey.

In contrast to well-established synthetic one-pot protocols towards all-pyrrole containing expanded porphyrins, ${ }^{[51]}$ synthesis of the Siamese-twin porphyrin $\mathbf{2 7}_{4}$ consists of a step-bystep design of crucial components.[76] It involves the three-step assembly of the 3,4,5-trisubstituted pyrazole $\mathbf{2 8}$ (Scheme 5) as a first key building block. [78] A further threestep transformation yields the pyrazole/pyrrole-hybrid $\mathbf{2 9}$ as a stereoisomeric mixture. Ethyl substitution at the $\beta$-positions of the pyrrole was chosen to ensure sufficient solubility for the subsequent concentration-sensitive cyclization and oxidation reactions to yield $\mathbf{2 7} \mathbf{H}_{\mathbf{4}}$. Bulky substitution in the 4-position of the pyrazole is essential to avoid rotational inversion of this subunit in the target molecule. Thus a preorganization of a $\left\{\mathrm{N}_{4}\right\}$-coordination pocket in $\mathbf{2 7} \mathbf{H}_{4}$ is assured. Aryl substitution at the side arms in $\mathbf{2 8}$ further proved beneficial for the oxidation of the meso-positions in the final step of the synthesis. It stands to reason that this challenging synthesis is not awarded with high yields. However, so far no alternative substitution pattern at the pyrazole/pyrrole-hybrid led to a successful isolation of another Siamese-twin porphyrin. ${ }^{[79]}$

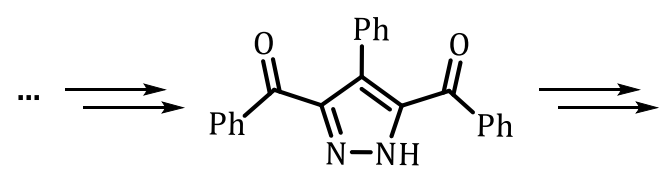

(28)

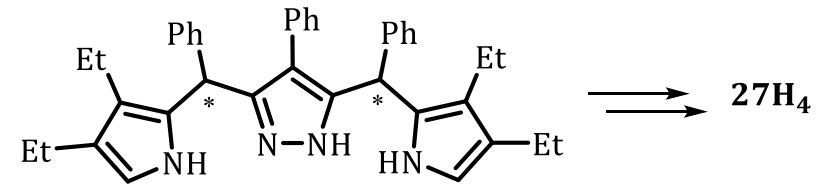

(29)

Scheme 5: Key intermediates in the synthesis towards the Siamese-twin porphyrin 27. 4 : 3,5-dibenzoyl-4phenyl-1H-pyrazole (28) and 3,5-bis-(3,4-diethyl-1H-pyrrole-2-yl-benzyl)-1H-pyrazole (29).[76,78,80]

During the investigation of the free ligand, solely the doubly protonated molecule (Figure 7, $\mathbf{2 7}_{\mathbf{6}}{ }^{2+}$ ) could be crystallized and its solid state structure has shown a severe macrocyclic twist. ${ }^{[76]}$ Contrary to the idealized drawing of $\mathbf{2 7} \mathbf{H}_{\mathbf{4}}$ in Scheme 4 , the $\left\{\mathrm{N}_{4}\right\}$-coordination pockets are not arranged co-planarly, but tilted at an angle of approximately $\tau=41^{\circ}$ with respect to the mean planes of each pocket. Calculations revealed that the twist originates from the bulky peripheral substitutions along the long side of the molecule. All synthetic attempts to minimize the strain thus far have proven unsuccessful due to either the lack of appropriate starting building blocks or the dependence of the final oxidation step on the nature of the substitution pattern.[79] 

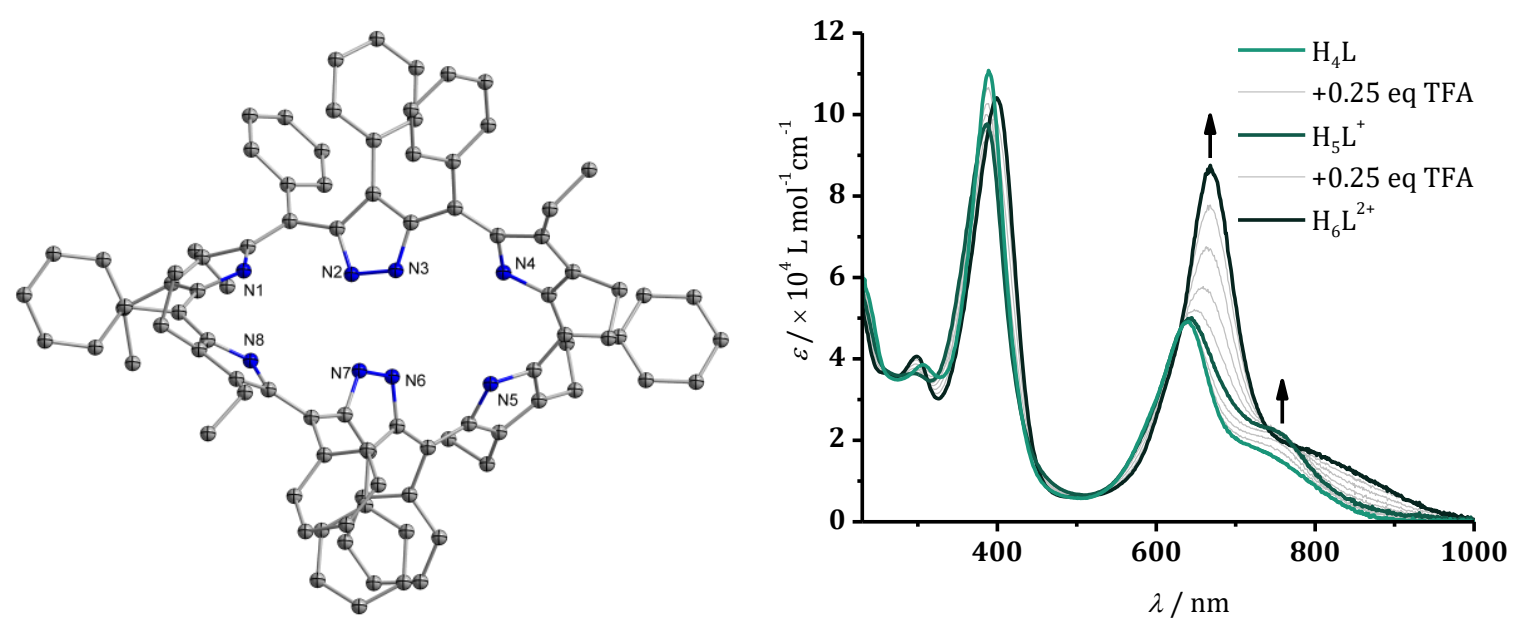

Figure 7: On the left, ball-and-stick representation of the solid state structure of doubly protonated Siamese-twin porphyrin $\mathbf{2 7} \mathbf{H}_{\mathbf{6}}{ }^{\mathbf{2 +}}$ (carbon in grey, nitrogen in blue). Only one enantiomer shown; solvent molecules, counter ions and hydrogen atoms omitted for clarity. The figure was reproduced from the structure deposited at the Cambridge Crystallographic Data Center: CCDC 906753.[76] On the right, protonation titration of Siamese-twin porphyrin $\mathbf{2 7} \mathbf{H}_{4}$ (teal trace) monitored by UV/vis $\left(298 \mathrm{~K}, \mathrm{CH}_{2} \mathrm{Cl}_{2}\right)$. Mono- $\left(\mathbf{2 7} \mathbf{H}_{5}{ }^{+}\right.$, dark-green trace $)$and diprotonated species $\left(\mathbf{2 7} \mathbf{H}_{6}{ }^{2+}\right.$, black trace) were detected upon addition of one and two equivalents of acid (TFA), respectively.[76]

A recent study by MITEVSKI reported on the variation of the phenyl substituents with different aryl groups at the meso-pyrrole/pyrrole position. No significant effects on the topology of the macrocycle could be observed and merely slight electronic effects related to the respective HAMMETT-parameters of the aryl substituents could be detected.[81]

The helical twist along the long axis of the molecule renders the macrocycle chiral. This intrinsic feature is common among figure-eight and twisted conformations of expanded porphyrins, but in most cases the two helical enantiomers interconvert rapidly and may not be separated.[60,63] While attempts to separate the neutral free-base Siamese-twin porphyrin $\mathbf{2 7} \mathbf{H}_{4}$ failed, two-fold protonation $\left(\mathbf{2 7}_{\mathbf{6}} \mathbf{2}^{\mathbf{2}}\right.$, Figure 7) allowed subsequent chiral resolution of the two enantiomers by HPLC on a chiral stationary phase. Lifetimes of $12 \mathrm{~h}$ for $\mathbf{2 7} \mathbf{H}_{4}$ and $4 \mathrm{~d}$ for $\mathbf{2 7}_{\mathbf{6}}{ }^{\mathbf{2}^{+}}$pointed to rather slow enantiomeric interconversion rates.[76]

\subsubsection{Metalation of the Siamese-Twin Porphyrin}

As observed for other expanded porphyrins (cf. Chapter 1.3.4), also the Siamese-twin porphyrin $\mathbf{2 7 H}_{4}$ readily forms complexes with $\mathrm{Ni(II)}$ and $\mathrm{Cu}(\mathrm{II})$ ions.[75,76,82] These complexes represent an ideal platform for initial and comparative analyses on the complexation behavior, coordination geometries and associated spectroscopic features. Reports by BLUSCH on the interaction of the macrocycle with its central ions define our current understanding of the electronic nature of this one-of-a-kind expanded porphyrin.[82,83]

To date several homo- and heterobimetallic complexes, as well as one monometallic, have been synthesized and characterized: $\mathbf{2 7} \mathbf{H}_{\mathbf{2}} \mathbf{N i}$ (Figure 8, left), $\mathbf{2 7} \mathbf{N i}_{2}, \mathbf{2 7} \mathbf{C u}_{\mathbf{2}}$ (as $\mathbf{2 7} \mathbf{C u}_{\mathbf{2}^{2+}}$ in Figure 8, right), and $\mathbf{2 7} \mathbf{F e}_{2} \cdot{ }^{[75,76,79,82-84]}$ All these complexes retain the macrocyclic twist and separation of both hemi-macrocyclic conjugation pathways as already observed for the

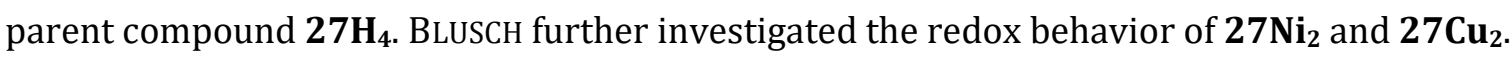
Complementary analyses led to reason that the Siamese-twin porphyrin is a redox noninnocent ligand and interacts readily with the coordinated metal ion upon oxidation by one 
or two electrons. Hereby, each individual coordination pocket is oxidized once with a difference of oxidation potential of approximately $\Delta E=350 \mathrm{mV}$ in the case of $\mathbf{2 7} \mathbf{C u}_{2 .}{ }^{\left[{ }^{[3]}\right]}$ Each ligand subunit forms a ligand-centered radical upon oxidation, though the spin vanishes because of covalency and strong antiferromagnetic coupling between the ligand radical and the proximate metal ion. Evaluation of bond lengths in the oxidation product suggested the radical being mainly delocalized upon the dipyrromethene subunit of the respective coordination pocket.[83]
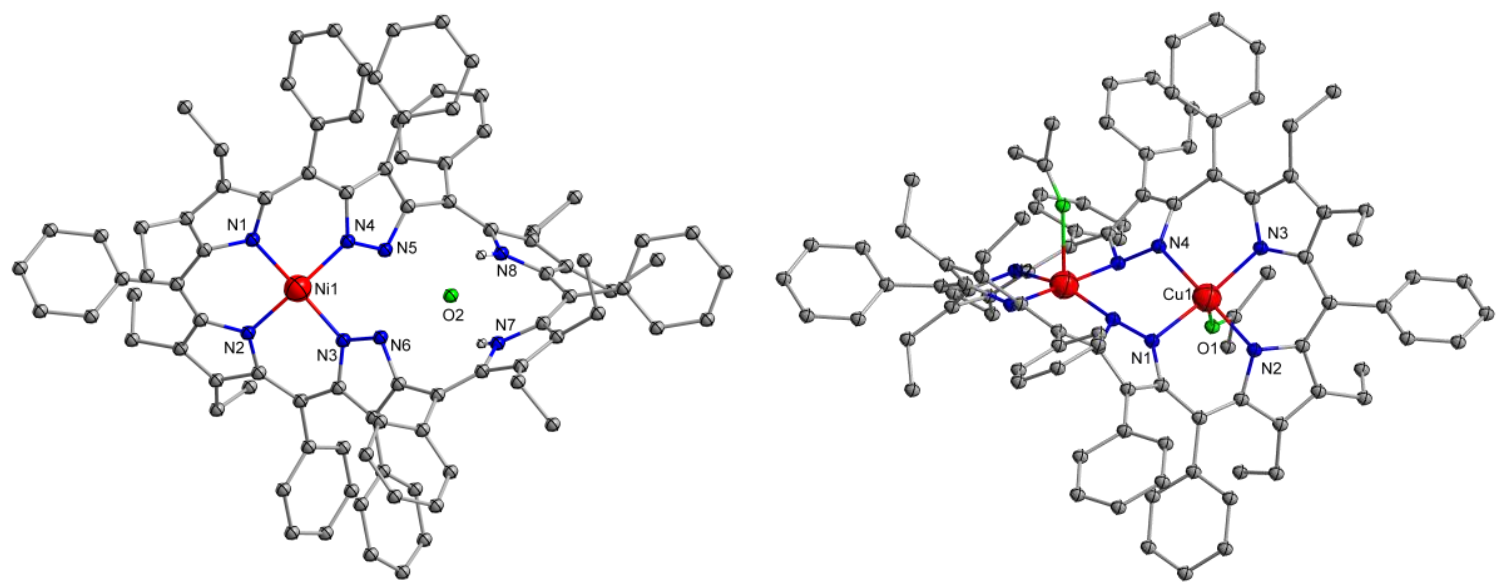

Figure 8: Ball-and-stick representations of the solid state structure of mononickel and doubly oxidized dicopper complexes of the Siamese-twin porphyrin $\mathbf{2 7 H _ { 2 }} \mathbf{N i}$ (left) and $27 \mathbf{C u}_{2}{ }^{2+}$ (right) (carbon in grey, nitrogen in blue, nickel/copper in red, oxygen in green and hydrogen in white). Only one enantiomer shown; solvent molecules, counter ions and carbon-bound hydrogen atoms omitted for clarity. Figures were reproduced from structures

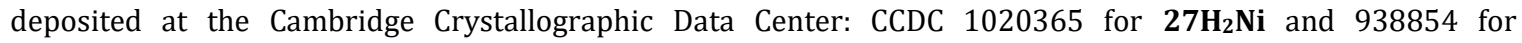
$27 \mathrm{Cu}_{2}{ }^{2+} \cdot[82,83]$

In addition to the non-innocent behavior of the ligand, recently prepared diferric complexes of the Siamese-twin porphyrin $\left(\mathbf{2 7} \mathbf{F e}_{2}\right)$ revealed another remarkable feature of this unusual macrocycle. The square-planar arrangement of the ligand with an additional axially coordinated chloride ion to give an overall square-pyramidal coordination sphere promotes the stabilization of an intermediate spin ground state $\left(S=\frac{3}{2}\right)$ on the central ion.[85] Previous studies suggested a correlation of geometric and electronic properties and trace major impacts on the electronic ground state to the degree of distortion in the macrocyclic backbone.[86] Synthetic models with ferric, chloride coordinating and sterically crowded porphyrin complexes supported that claim with reports on admixtures of different spin states $\left(S=\frac{5}{2}\right.$ and $\left.\frac{3}{2}\right) \cdot[86,87]$

Hitherto, all reports focus mainly on analyses of bimetallic complexes or the doubly protonated Siamese-twin porphyrin while structural evidence for the free-base porphyrin as well as analyses on its redox-behavior are still missing. Moreover, no monometalated species has been investigated towards its redox properties in detail yet. 



\section{OBJECTIVE}

Building upon the importance of porphyrins in nature as well as the rising involvement of porphyrinoid systems in catalytic applications, sensors or biomedical applications, a vast variety of new structural motifs with inspiring properties have been synthesized.

This work is based on the recent achievements in the synthesis of the Siamese-twin porphyrin and the first examples of its homo- and heterobimetallic complexes. The hitherto reported copper and nickel complexes provided a basic understanding on this novel system, whereas the synthesis of iron complexes moved the research closer to biomimetic aspects.

The aim of this work is to study the redox behavior of the Siamese-twin porphyrin itself (Scheme 6). On the one hand, a fully conjugated, macrocycle aromatic molecule is targeted. To achieve this, the macrocyclic scaffold is to be oxidized (Chapter 3.1) or derivatized (Chapter 3.2) in such a manner that a macrocycle aromatic system is implemented. On the other hand, metal complexes of the Siamese-twin porphyrin with redox-active and redoxinert metal ions are to be prepared and electrochemically analyzed (Chapters 3.3 and 3.4). The focus hereby is set on the understanding of cooperative effects of the central ion and the multi-electron platform allocated by the non-innocent ligand framework.
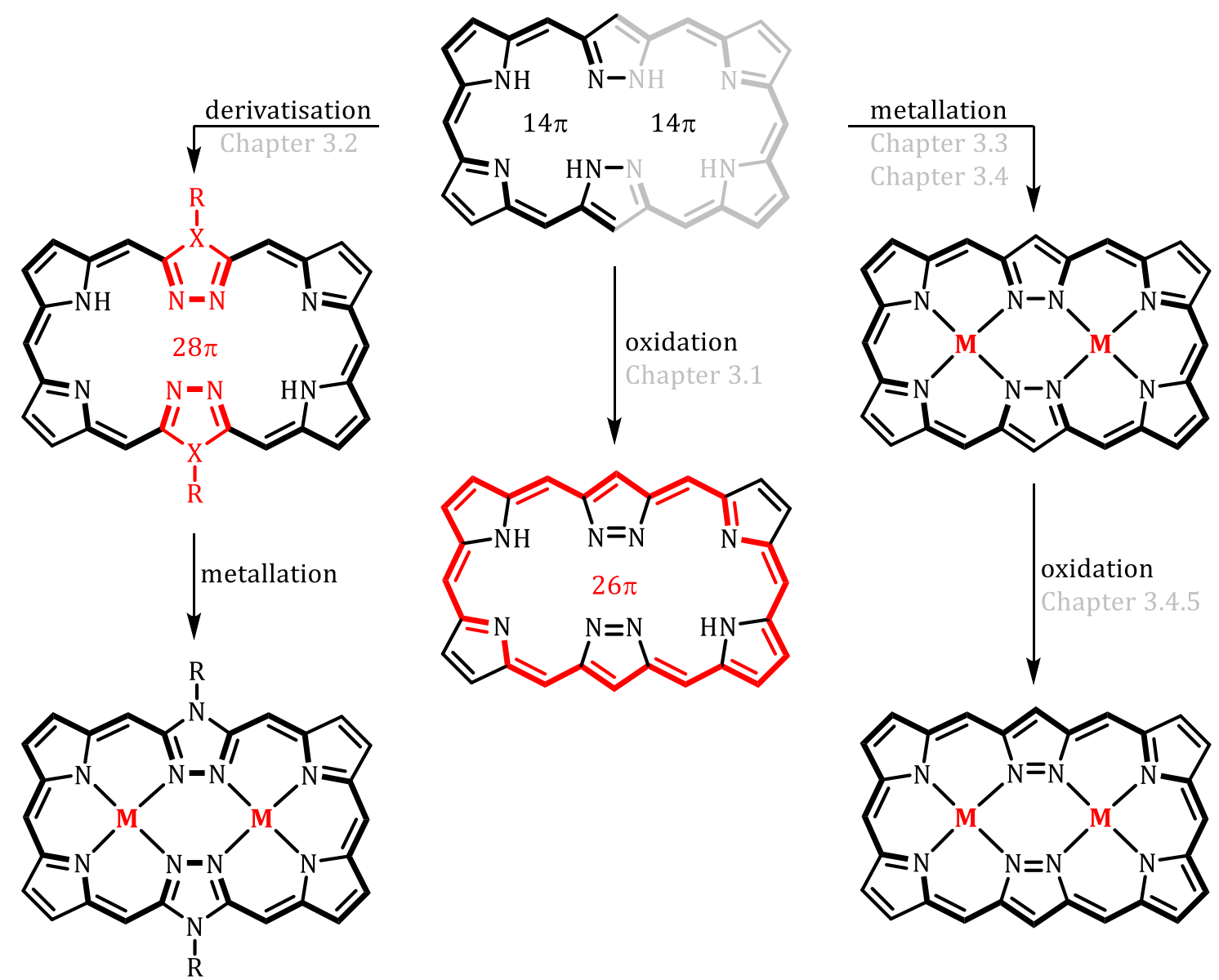

Scheme 6: General synthesis concept to the redox properties investigation of the Siamese-twin porphyrin $\mathbf{2 7} \mathbf{H}_{\mathbf{4}}$, its derivatives, and metal complexes (peripheral substituents are omitted for clarity). 
As described in the general introduction of this work, the initial insertion of a pyrazole moiety into the macrocyclic scaffold allowed to implement the merging of two porphyrinoid subunits in an elegant way. However, the same pyrazole subunit is accountable for the cut in the global conjugation pathway. To circumvent the interruption a modification of the electronic structure at the central unit without changes in the overall connectivity is favorable. Variations in the substitution pattern and introduction of a different heterocycle such as a triazole were chosen during the development of this project (Chapter 3.2). In doing so, the main challenge in the derivatization of the ligand lies in the synthesis of suitable precursors and the coupling to the corresponding pyrrole hybrids.

Another option to achieve full macrocyclic conjugation is a (further) oxidation of the given pyrazole subunit. Formal elimination of two electrons and two protons may yield a $26 \pi$ electron-bearing macrocycle (Chapter 3.1). Oxidation of the ligand demands careful consideration of reaction conditions since decomposition is likely.[79] Moreover, an educated choice of purification methods due to the expected close similarity of the parent molecule and the desired product is crucial.

Further, this work targets the synthesis of palladium and manganese complexes of the Siamese-twin porphyrin. Introduction of a $4 \mathrm{~d}$ metal ion with a high preference for squareplanar coordination geometry and greater redox-inertness in comparison to $\mathrm{Ni}$ and $\mathrm{Cu}$ is considered beneficial for a more detailed analysis of the nature of cationic and anionic radical species of the Siamese-twin porphyrin complexes (Chapter 3.4). Additionally, the increased ionic radius of the palladium ion makes it possible to test the boundaries of the coordination pocket for its size and flexibility.

Manganese distinguishes itself as a multivalent, redox-active metal ion with several easily accessible and stable redox states and coordination geometries. Many studies rendered manganese porphyrin complexes particularly susceptible to the formation of high-valent oxoand nitrido-species. Thus, manganese is included in this study with a focus towards the synthesis of suitable complex precursors and subsequent transformation into high-valent species (Chapter 3.3).

Investigations of the metal complex properties require a previous, profound understanding of the free-base Siamese-twin porphyrin. The outcome of metalation reactions of expanded porphyrins is not always predictable and so far, established synthetic routes may require reevaluation upon insertion of new metal ions. The desired cooperation of the metal ion and the non-innocent ligand imposes an additional challenge in the interpretation of analytical data and demands careful comparison with literature. Combination of complimentary analytical techniques will be indispensable to substantiate conclusions. 


\section{RESULTS AND DISCUSSION}

\subsection{Oxidation}

\subsubsection{Siamese-Twin Porphyrin Oxidation}

Building upon the rich redox chemistry of different expanded porphyrins (compare Chapter 1.3) as well as on the redox behavior of complexes of the Siamese-twin porphyrin described by BLUSCH,[82,83] a $26 \pi$-electron macrocycle aromatic molecule $\left(\mathbf{3 0 H}_{2}\right.$, Scheme 7$)$ was postulated. The results on the oxidation of the Siamese-twin porphyrin $\mathbf{2 7} \mathbf{H}_{\mathbf{4}}$ presented in the following chapters were already summarized in a publication. ${ }^{[88]}$

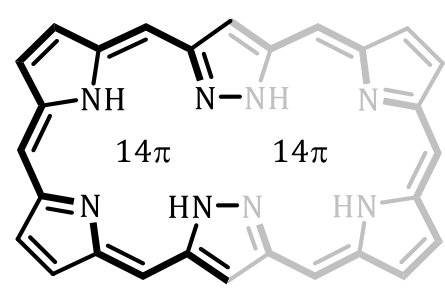

$\left(27 \mathrm{H}_{4}\right)$

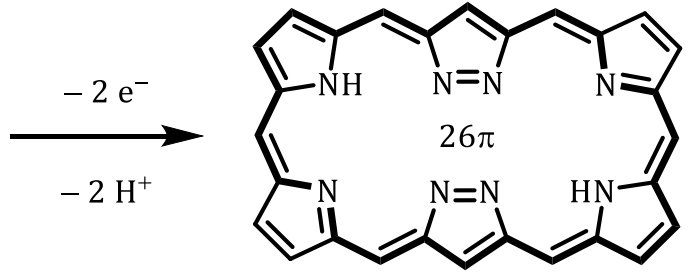

$\left(30 \mathrm{H}_{2}\right)$

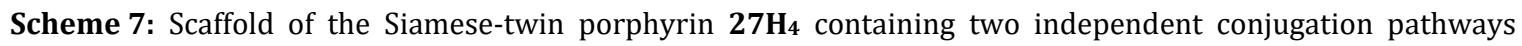
(highlighted in bold) and a targeted macrocycle with overall aromatic circuit upon oxidation (30H2); peripheral substituents omitted for clarity.

The Siamese-twin porphyrin $\mathbf{2} \mathbf{2 H}_{\mathbf{4}}$ is obtained by an acid-catalyzed [3+3] condensation reaction according to the FISCHER and ROTHEMUND approach (cf. Experimental Section). The cyclization reaction is carried out with equimolar solutions of the pyrazole/pyrrole-hybrid 29 (Scheme 5), benzaldehyde and trifluoroacetic acid (TFA). Subsequent oxidation of the obtained Siamese-Twin porphyrinogen $\left(\mathbf{3 1 H}_{\mathbf{6}}\right.$; not shown) with four equivalents of the twoelectron oxidant 2,3-dichloro-5,6-dicyano-1,4-benzochinone (DDQ) ${ }^{[89]}$ yield the Siamese-twin porphyrin $\mathbf{2 7} \mathbf{H}_{4}$.

Oxidation of the Siamese-Twin porphyrinogen $\mathbf{3 1 H}_{\mathbf{6}}$ with increasing amounts ( 5 and 6 eq.) of DDQ led to mainly decomposition products and lower yields of the known Siamese-twin porphyrin $\mathbf{2} \mathbf{7 H}_{4}$ in comparison to the initial conversion with stoichiometric amounts (4 eq.) of the oxidizing agent. However, subsequent experiments with the isolated and purified Siamese-twin porphyrin as the reactant and carefully controlled amounts of oxidant under milder conditions proved successful (Scheme 8).

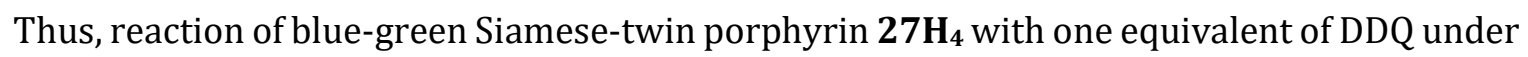
inert conditions over two days at slightly elevated temperatures $\left(\mathrm{CH}_{2} \mathrm{Cl}_{2},<40{ }^{\circ} \mathrm{C}\right)$ led to a slightly greener color of the reaction mixture. TLC indicated the formation of a new, brightgreen product $\left(\mathbf{3 2 H}_{3}\right)$ of lesser polarity $\left(R_{\mathrm{f}}=0.76\right.$ vs $R_{\mathrm{f}}=0.54$ for $\mathbf{2 7 \mathbf { H } _ { 4 }}$; both neutral alumina, $n$-hexane/THF/EtOAc, 20:3:1). Product $\mathbf{3 2} \mathbf{H}_{3}$ was isolated in $13 \%$ yield by preparative TLC (same conditions as analytical TLC), followed by purification on a SEPHADEX column (LH-20, $\mathrm{MeOH})$. The composition, as determined by HR-MS $\left(\mathrm{ESI}^{+}\right)$, suggested that $\mathbf{3 0 H}_{3}$ is a twohydrogen-atom oxidation product of $2 \mathbf{2 7}_{4}\left(\mathrm{C}_{92} \mathrm{H}_{83} \mathrm{~N}_{8}{ }^{+}\right.$for $\mathrm{MH}^{+}$; Figure 9). 


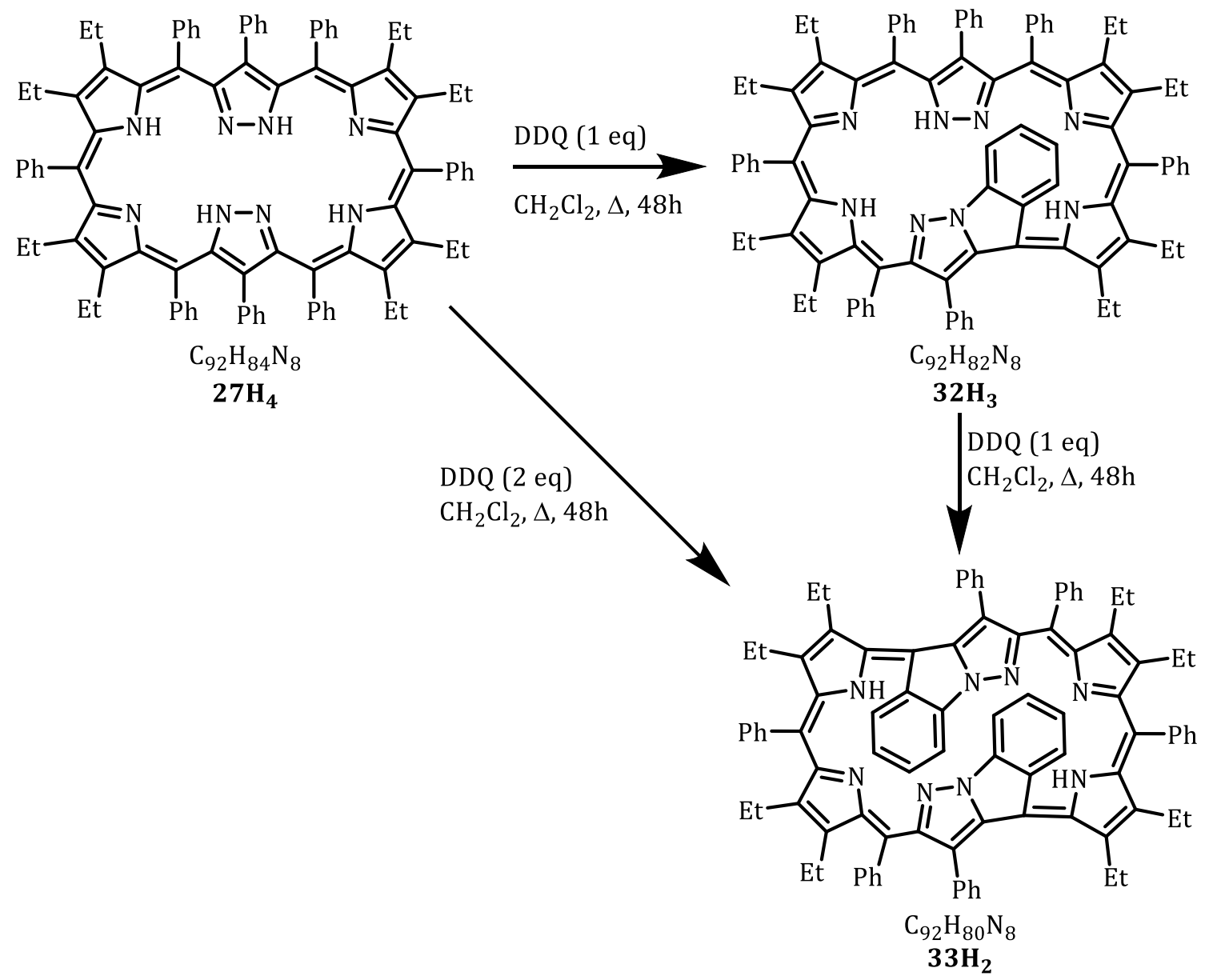

Scheme 8: Two- and four-hydrogen oxidations of Siamese-twin porphyrin $27 \mathbf{H}_{4}$.

The synthetic conditions towards $\mathbf{3 2} \mathbf{H}_{3}$ were optimized by heating the reaction mixture in $1,2-\mathrm{C}_{2} \mathrm{H}_{4} \mathrm{Cl}_{2}$ at $80^{\circ} \mathrm{C}$ over $2 \mathrm{~h}$ affording similar yields. Addition of solid, dry $\mathrm{K}_{2} \mathrm{CO}_{3}$ to the reaction mixture proved to be beneficial to assure reproducibility of the reaction. This is

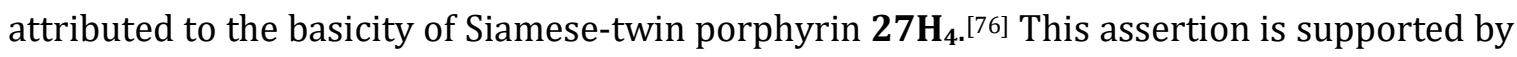
control experiments in which the oxidation was performed in the presence of acid (2 eq TFA), and in which no conversion of $\mathbf{2} \mathbf{7} \mathbf{H}_{4}$ took place.
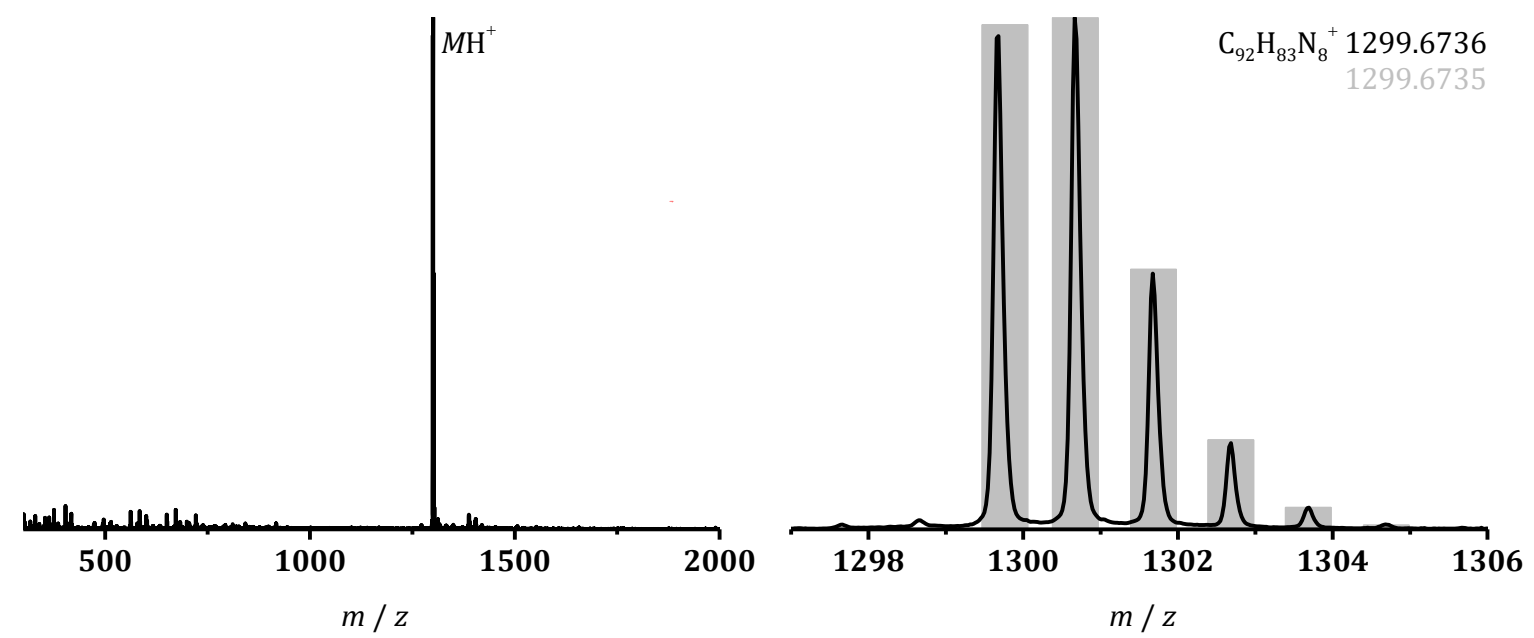

Figure 9: $\mathrm{HR}_{-\mathrm{ESI}}{ }^{+}$spectrum of $\mathbf{3 2} \mathbf{H}_{3}$ and the isotopic distribution of the molecular peak $\mathrm{MH}^{+}$(simulation in grey). 
The oxidation of Siamese-twin porphyrin $\mathbf{2} \mathbf{7} \mathbf{H}_{\mathbf{4}}$ with two equivalents of DDQ was carried out under the same conditions. The blue-green color of the bulk mixture changed to olive-green. Initially, TLC of the crude reaction mixture showed only two spots corresponding to the starting material $\mathbf{2} \mathbf{2 H}_{\mathbf{4}}$ and the first oxidation product $\left(\mathbf{3 2} \mathbf{H}_{3}\right)$, but eventually a third highpolarity product $\left(R_{\mathrm{f}}=0.22\right.$, conditions as above; $\mathbf{3 3 \mathbf { H } _ { 2 }}$ ) appeared. Over time, the quantity of

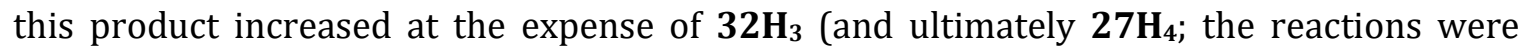
stopped after about $\mathbf{8 0} \%$ of $\mathbf{2} \mathbf{7 H}_{4}$ were converted). Product $\mathbf{3} 3 \mathbf{H}_{2}$ was isolated in $8 \%$ yield in a similar way as described for $\mathbf{3 2} \mathbf{H}_{3}$, followed by crystallization from $\mathrm{CH}_{2} \mathrm{Cl}_{2}$ layered with $\mathrm{MeOH}$. The HR-MS spectrum (ESI ${ }^{+}$) of $\mathbf{3 3} \mathbf{H}_{\mathbf{2}}$ indicated that the composition corresponds to a four-hydrogen-atom oxidation product of $\mathbf{2 7} \mathbf{H}_{4}\left(\mathrm{C}_{92} \mathrm{H}_{81} \mathrm{~N}_{8}{ }^{+}\right.$for $M \mathrm{H}^{+}$; Figure 10). As expected, oxidation of the two-hydrogen product $\mathbf{3 2 H}_{3}$ with a single equivalent of DDQ also generated compound $33 \mathbf{H}_{2}$.
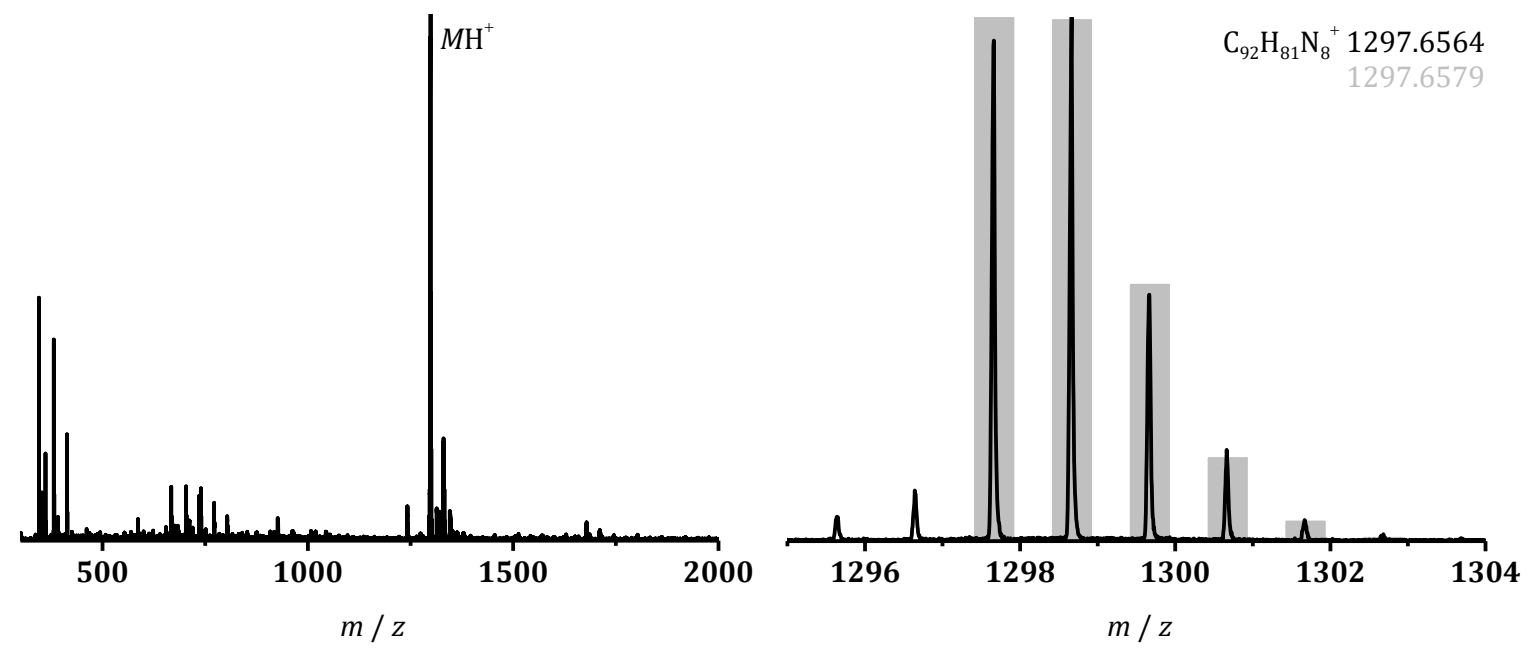

Figure 10: $\mathrm{HR}_{-\mathrm{ESI}^{+}}$spectrum of $\mathbf{3} 3 \mathbf{H}_{\mathbf{2}}$ and the isotopic distribution of the molecular peak $\mathrm{MH}^{+}$(simulation in grey).

It should be pointed out that although oxidative conditions were applied, working under strictly inert conditions was crucial to the success of the reaction. This fact in attributed to sensitive potential changes of the oxidant upon contact with $\mathrm{H}_{2} \mathrm{O}$ as well as possible aerial recovery of the oxidant and thus overall loss of stoichiometry control. So far no other oxidizing agent (e.g. $\mathrm{FeCl}_{3}, \mathrm{H}_{2} \mathrm{O}_{2}, \mathrm{Ag}_{2} \mathrm{O}$ or TEMPO) was proven suitable for the reaction.

\subsubsection{UV/vis Spectral Characterization}

Both oxidation products $\mathbf{3 2} \mathbf{H}_{\mathbf{3}}$ and $\mathbf{3 3 \mathbf { H } _ { 2 }}$ as well as the parent molecule $\mathbf{2 7} \mathbf{H}_{\mathbf{4}}$ are intensively green (Figure 11, right). All molecules express a SORET-like band at shorter wavelengths $(\lambda \approx 400 \mathrm{~nm}$; Figure 11$)$ and a broad and poorly defined absorption at longer wavelengths $(\lambda>600 \mathrm{~nm})$. UV/vis spectra of the oxidation products $\mathbf{3 2} \mathbf{H}_{3}$ and $\mathbf{3 3 \mathbf { H } _ { 2 }}$ show that the two successive oxidations lead to redshifts in both prominent absorption bands relative to the spectrum of the Siamese-twin porphyrin $\mathbf{2} \mathbf{7 H}_{4}$, with little other fundamental changes (Table 3).

One notable feature in the optical spectrum of $\mathbf{3} 3 \mathbf{H}_{2}$, when compared to the parent compound

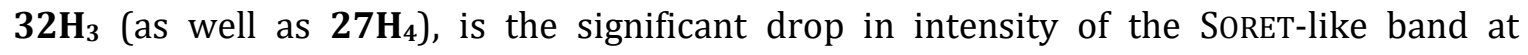
$\lambda=435 \mathrm{~nm}$, suggestive of considerable conformational changes upon oxidation of $\mathbf{3 2} \mathbf{H}_{3}$. The molar extinction coefficients for the main bands of all three compounds fall in the range of 

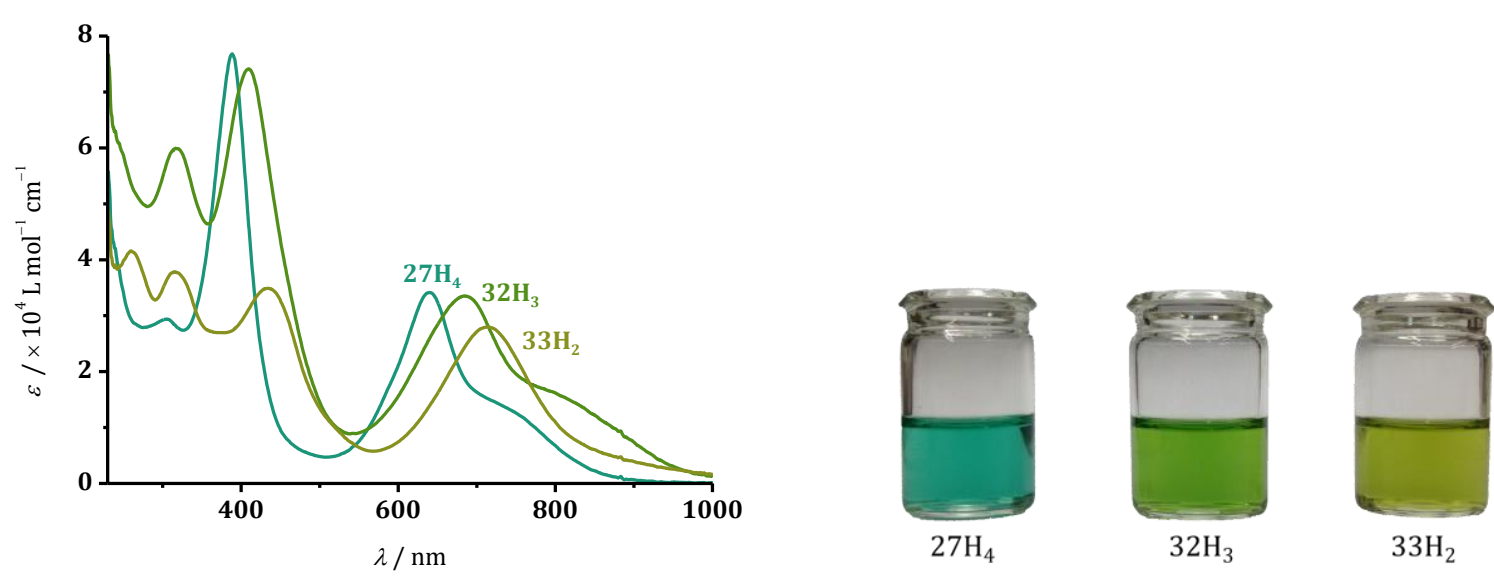

Figure 11: UV/vis spectra (298 $\mathrm{K}, \mathrm{CH}_{2} \mathrm{Cl}_{2}$ ) of Siamese-twin porphyrin $\mathbf{2 7} \mathbf{H}_{4}$ (teal trace), two-hydrogen oxidation product $\mathbf{3 2} \mathbf{H}_{3}$ (green trace), and the four-hydrogen oxidation product $\mathbf{3 3} \mathbf{H}_{2}$ (olive trace).

$10^{4} \mathrm{~L} \mathrm{~mol}^{-1} \mathrm{~cm}^{-1}$, consistent with the notion that the non-aromatic chromophore structure of the Siamese-twin porphyrin $\mathbf{2} \mathbf{7 H}_{\mathbf{4}}$ was principally maintained upon oxidation. The extinction coefficients, which are significantly lower than for aromatic porphyrins, ${ }^{[90]}$ also fit well with comparable examples of (anti)aromatic expanded porphyrins, such as the [26]- and [28]hexaphyrins.[54,90] Nonetheless, the absence of any significant change in the optical spectra suggests that the anticipated target macrocycle structure $\mathbf{3 0 H}_{2}$ was not achieved, and that an alternative oxidation must have taken place.

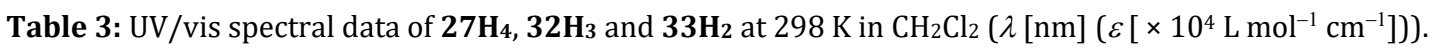

\begin{tabular}{ccc}
\hline $\mathbf{2 7 \mathbf { H } _ { \mathbf { 4 } }}$ & $\mathbf{3 2 H}_{\mathbf{3}}$ & $\mathbf{3 3 H}_{\mathbf{2}}$ \\
\hline- & - & $260(4.2)$ \\
$305(2.9)$ & $319(6.0)$ & $316(3.8)$ \\
$389(7.7)$ & $409(7.4)$ & $435(3.5)$ \\
$641(3.4)$ & $685(3.4)$ & $716(2.8)$ \\
$740(1.3)$ & $830(1.4)$ & - \\
\hline
\end{tabular}

Since conformational changes and therewith associated absorptive properties may be temperature-dependent, variable temperature UV/vis spectroscopy was conducted. Absorption spectra of the oxidation products $\mathbf{3 2} \mathbf{H}_{3}$ and $\mathbf{3 3} \mathbf{H}_{2}$ remained essentially unchanged over the temperature range of $+20^{\circ} \mathrm{C}$ to $-90^{\circ} \mathrm{C}$ (Figure 12), demonstrating the relative conformational rigidity of the chromophore. A marginal increase in the extinction coefficient of all major features is observed, as expected for a higher ground state population with decreasing temperature. In addition, lack of significant changes excludes the possibility of aromaticity switching upon cooling as seen for [28] hexaphyrins.[41,46] 

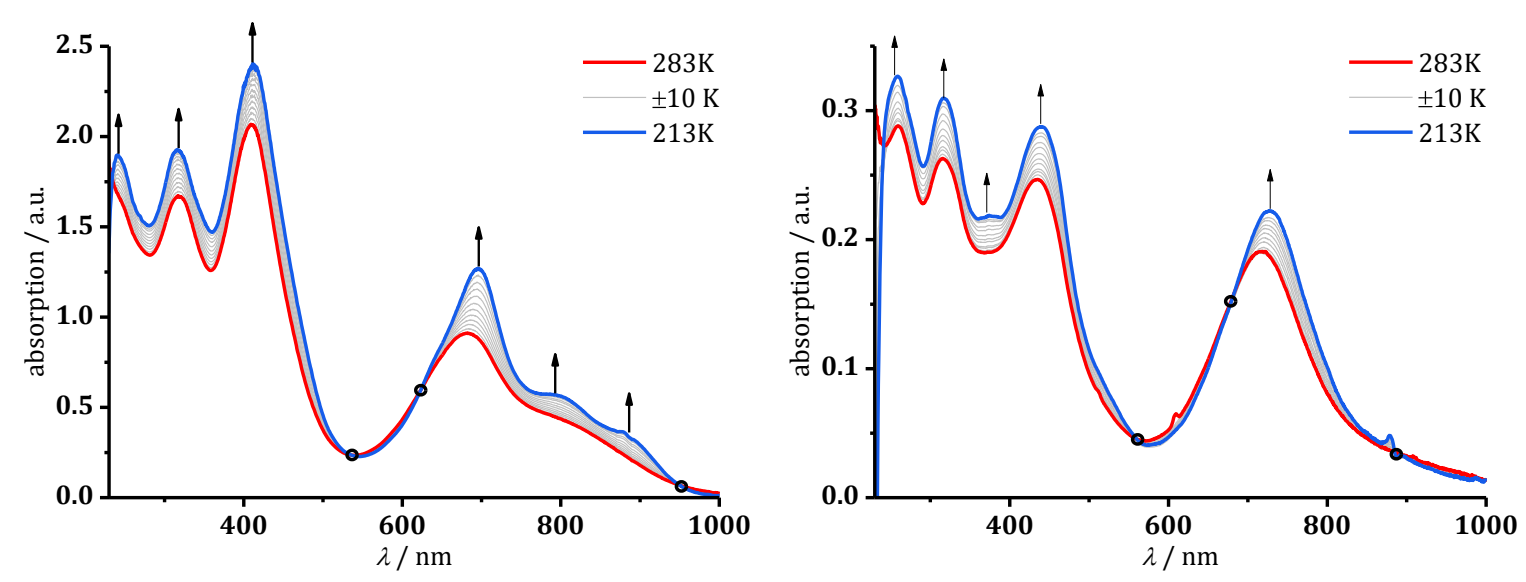

Figure 12: Variable temperature UV/vis spectra of $\mathbf{3 2} \mathbf{H}_{3}$ (left) and $\mathbf{3 3} \mathbf{H}_{2}$ (right) in the temperature range of $+20^{\circ} \mathrm{C}$ to $-90{ }^{\circ} \mathrm{C}$ (fully reversible changes upon cooling are indicated with arrows; isosbestic points are encircled).

\subsubsection{X-ray Crystallographic Molecular Structures}

While the structure of the oxidation products $\mathbf{3 2} \mathbf{H}_{3}$ and $\mathbf{3 3} \mathbf{H}_{2}$ may be deduced by NMR spectroscopy with a high level of confidence (Chapter 3.1.4), a definitive structure was determined by single crystal X-ray diffractometry. However, to facilitate the discussion of the NMR spectra, crystallographic details as proof of the structure and conformation are presented first.

Hitherto, structural evidence for the Siamese-twin porphyrin $\mathbf{2 7} \mathbf{H}_{4}$ was reported by BLUSCH

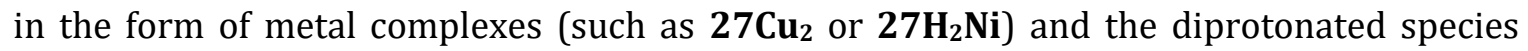
$\mathbf{2 7}_{\mathbf{6}} \mathbf{2}^{\mathbf{2}}$. However, no crystal structure of the otherwise well-characterized free-base parent compound $\mathbf{2 7 \mathbf { H } _ { 4 }}$ has been reported to date.[76] For a better understanding of product formation upon oxidation, the molecular structure of $\mathbf{2} \mathbf{7} \mathbf{H}_{4}$ is presented up-front.

Single crystals of Siamese-twin porphyrin $\mathbf{2 7} \mathbf{H}_{4}$ suitable for X-ray diffraction were obtained by slow evaporation of a saturated solution of $\mathbf{2 7} \mathbf{H}_{\mathbf{4}}$ in benzene. The data were resolved in the triclinic, non-chiral space group $P \overline{1}$, showing the presence of a racemic mixture of two helimeric conformers of $\mathbf{2 7} \mathbf{H}_{4}$ of pseudo- $C_{2}$-symmetry (non-crystallographic $C_{2}$-symmetry; Figure 13; Chapter 6.1).

The overall shape of the molecule can be described as a figure-eight (Figure 13C). In free-base $\mathbf{2 7} \mathbf{H}_{4}$, all pyrrole and pyrazole $\mathrm{NH}$ protons are involved in intramolecular hydrogen bonding, locking both dipyrromethene substructures in a nearly planar fashion. The ethyl substituents are pointing above and below the mean plane (as observed for $\beta$-ethylporphyrins).[91] Furthermore, as generally observed for meso-tetraaryl-substituted porphyrinoids, the phenyl groups assume an idealized perpendicular arrangement with respect to the mean plane of the neighboring porphyrinic subunits.[91,92]

One of the most striking structural features is the respective arrangement of substituents on the long side of the molecule: One of the meso-phenyl groups and the 4-pyrazolophenyl group point toward the outside of the macrocycle, while the remaining meso-phenyl group points more inward (Figure 13A; red-colored phenyl group in Figure 14; select phenyl groups were color-coded to facilitate the tracking of their movements). In comparison with the known helimeric conformation of diprotonated $\mathbf{2} \mathbf{7} \mathbf{H}_{\mathbf{4}}\left(\mathbf{2 7}_{\mathbf{6}} \mathbf{6}^{2+}\right),{ }^{[76]}$ the conformation of the free-base 


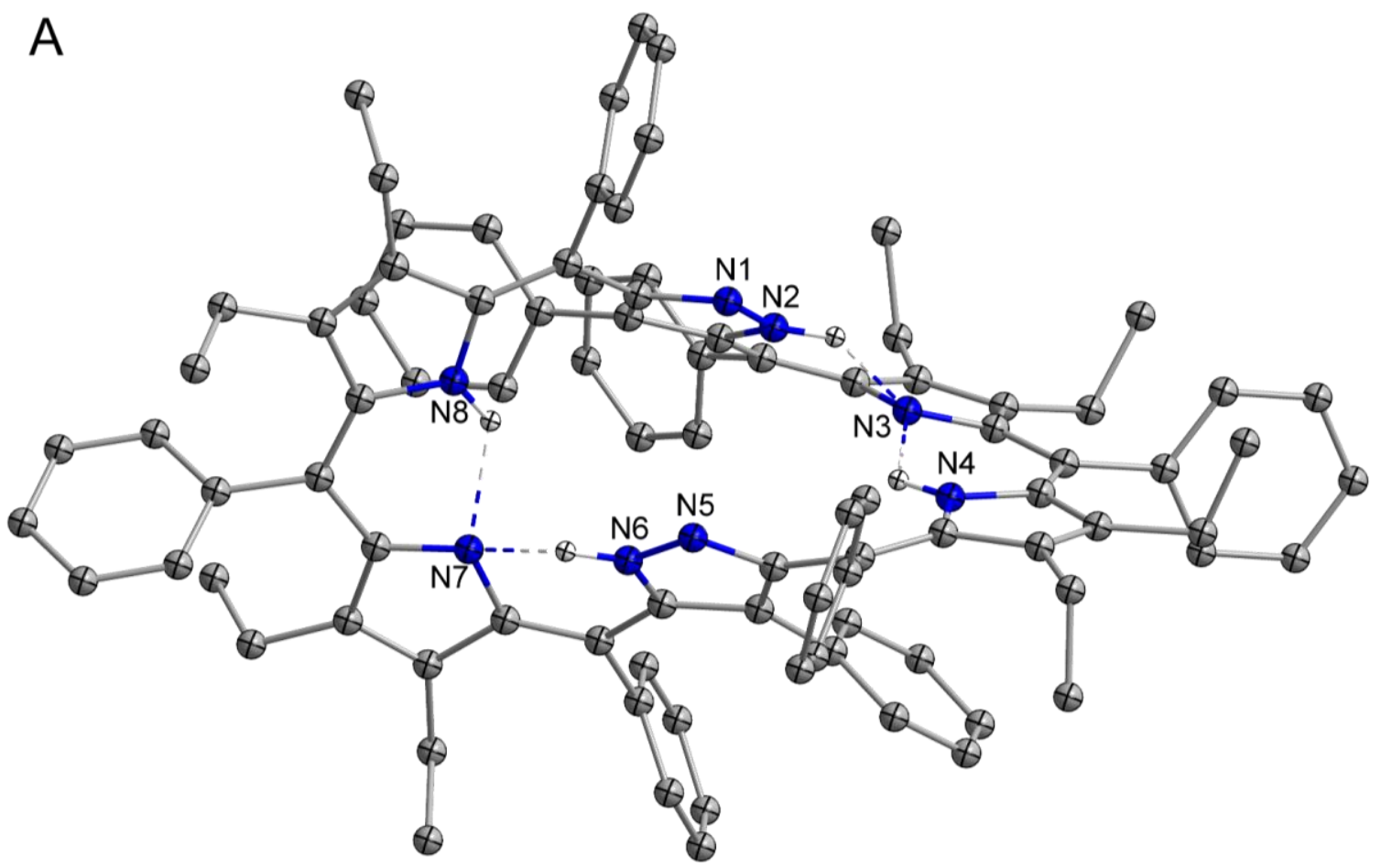

B
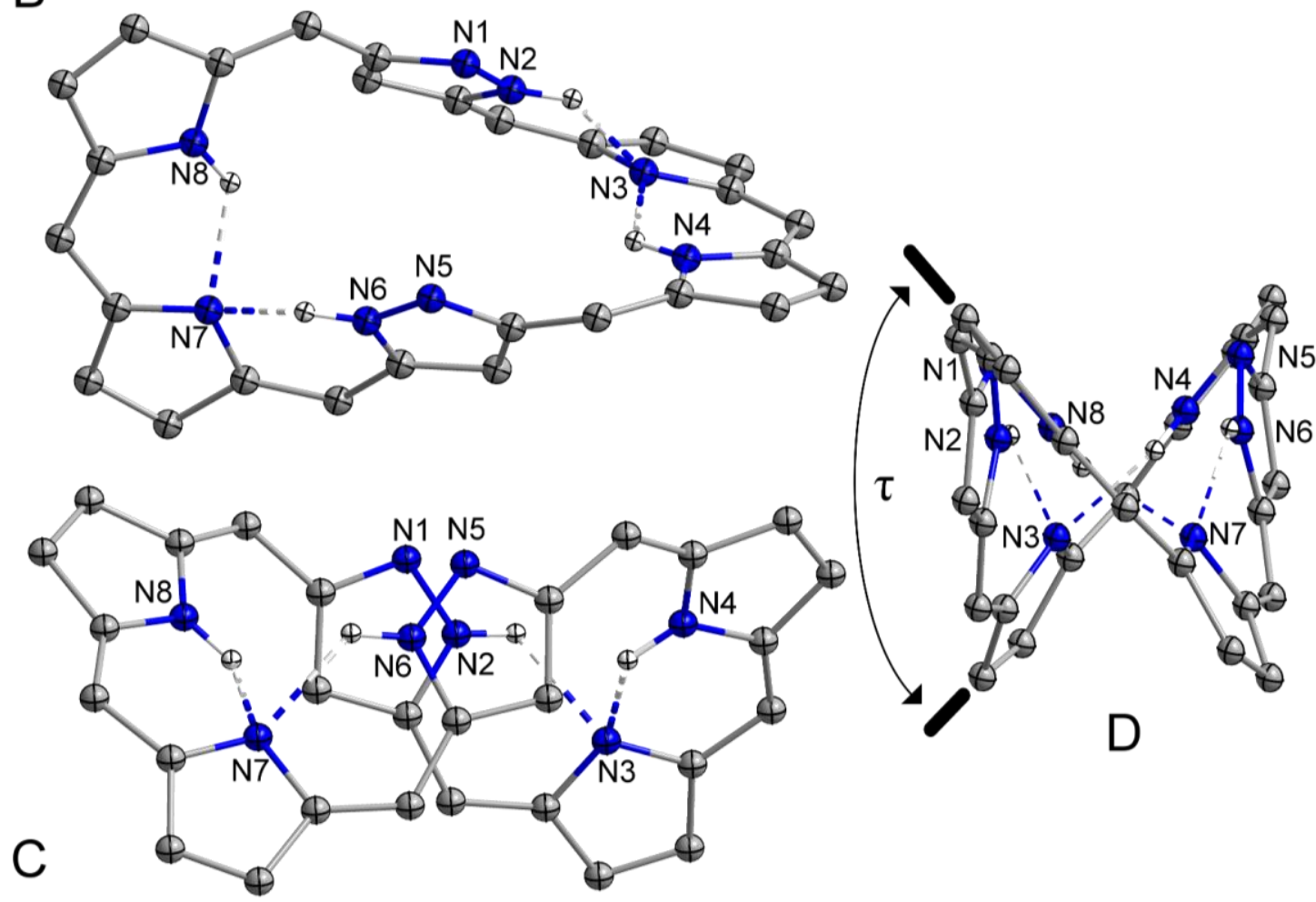

Figure 13: Ball-and-stick representation of the solid state structure of free-base Siamese-twin porphyrin $\mathbf{2 7 \mathbf { H } _ { 4 }}$ (carbon in grey, nitrogen in blue and hydrogen in white). Only one enantiomer shown; solvent molecules and carbon-bound hydrogen atoms omitted for clarity. A) Overview including peripheral substituents; B) representation of macrocycle conformation (same view as in A); C) representation of the pseudo- $C_{2}$-symmetry of the core structure highlighting the figure-eight topology of the core, and D) view along the long axis depicting the torsion angle $\tau$. 


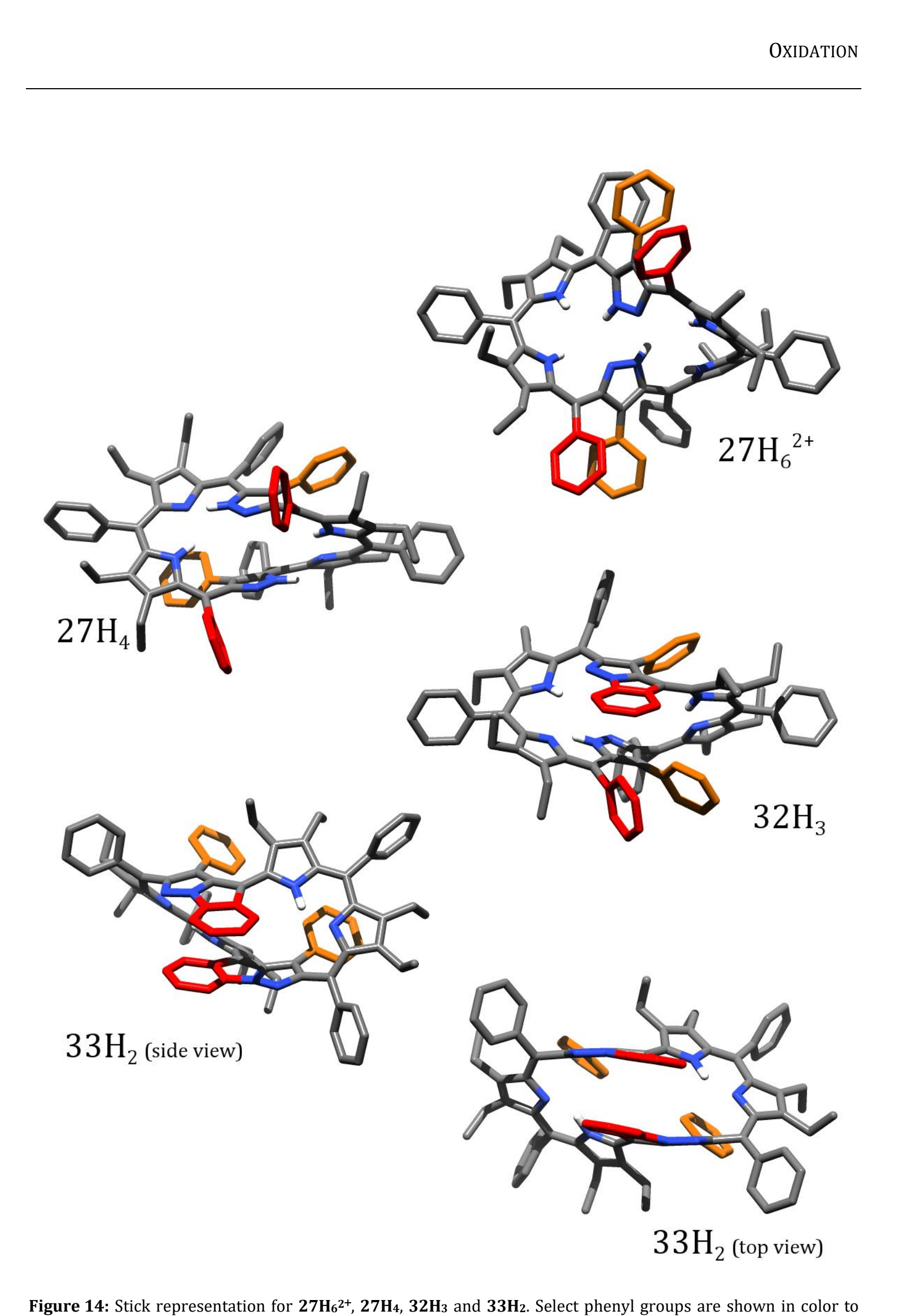


$\mathbf{2 7} \mathbf{H}_{4}$ is more distorted, with a helical pitch of $d_{\text {helix }}=10.78 \AA$ and a torsion angle (Figure 13D) of $\tau= \pm 97^{\circ}$ (for $2 \mathbf{2 7}_{\mathbf{6}^{2+}}{ }^{+}:{ }^{76]} d_{\text {helix }}=10.70 \AA$ and $\tau= \pm 92^{\circ}$ ). ${ }^{*}$ Evidently, protonation twists the pyrazole moiety inward by nearly $90^{\circ}$ to allow for H-bonding with the protonated dipyrrin moieties in $\mathbf{2} \mathbf{7 H}_{\mathbf{6}} \mathbf{2}^{\mathbf{+}}$ (Figure 14). This twisting motion and the electrostatic repulsion of the protonated nitrogen atoms, leads to a relative flattening of the twist inherent to the protonated structure; $\mathbf{2 7} \mathbf{H}_{\mathbf{6}}{ }^{\mathbf{2}}$ orients all meso- and pyrazole phenyl groups to point out of the macrocycle. Conversely, the free-base macrocycle lacks the grip on nitrogen atoms N1 and N5 via hydrogen bonds, thus allowing a pitch of the pyrazole moiety. This motion brings one meso-phenyl group attached to it from the outside of the ring to the inside, positioning one of its ortho- $\mathrm{CH}$ in relatively close vicinity of a nitrogen atom of the flanking pyrazole moiety $\left(d\left(\mathrm{~N} 5 \cdots \mathrm{Cortho}^{\mathrm{Ph}}\right)=3.30 \AA \AA\right.$ 品 Figure 14).

The pseudo-two-fold symmetric structure is fully consistent with the NMR data for $\mathbf{2 7} \mathbf{H}_{\mathbf{4}}$ (cf. Chapter 3.1.4). In contrast to the bond lengths observed in aromatic porphyrins and porphyrinoids, ${ }^{[49]}$ yet analogous to the observations made for the diprotonated form $\mathbf{2 7}_{\mathbf{6}} \mathbf{2}^{2+},{ }^{, 76]}$ the $\mathrm{C}-\mathrm{C}$ bond lengths of the framework of free-base $27 \mathbf{H}_{\mathbf{4}}$ exhibit the hallmarks of conjugated but not aromatic structures, namely, alternating short-long $\mathrm{C}-\mathrm{C}$ bonds $\left(d_{\max }(\mathrm{C}-\mathrm{C})=1.48 \AA, d_{\min }(\mathrm{C}-\mathrm{C})=1.36 \AA\right.$; cf. Chapter 6.1$)$. This also corresponds well with the interpretations of the UV/vis and NMR spectra of $\mathbf{2 7} \mathbf{H}_{\mathbf{4}}$ (and $\mathbf{2} \mathbf{2} \mathbf{H}_{\mathbf{6}}{ }^{\mathbf{2}}$ ).[76]

Single crystals of the two-hydrogen oxidation product $\mathbf{3 2} \mathbf{H}_{3}$ suitable for X-ray diffraction were obtained by slow evaporation of a saturated solution of $\mathbf{3 2} \mathbf{H}_{3}$ in acetone as a racemic mixture of two helimeric conformers. Resolved in the monoclinic, non-chiral space group $P 2_{1} / c$ (Figure 15; Chapter 6.1), the conformation of the macrocycle is partially planarized by a covalent linkage between the ortho-position of the long-side meso-phenyl group, which was already oriented inward in the macrocycle of $\mathbf{2} \mathbf{7} \mathbf{H}_{\mathbf{4}}$, and the neighboring pyrazole nitrogen atom (N5). This linkage induces an elongation of the helical pitch of the twisted conformation $\left(d_{\text {helix }}=10.88 \AA\right)$, together with larger torsion angles $\left(\tau= \pm 104^{\circ}\right)$.

The presence of the newly formed tricyclic pyrazolo[1,5- $a$ ]indole substructure lifts the pseudo- $C_{2}$-symmetry of the core of $\mathbf{2} \mathbf{7} \mathbf{H}_{4}$. The tricyclic structure enforces local rigidity on the respective long side of the molecule by removal of one of the former hinge points. Because the H-bonded tripyrrin units remain planar, the macrocyclic twist of the core is now expressed through the twisting of the bonds flanking the three remaining meso-positions. One mesophenyl group on the opposite long side of the tricyclic subunit still reaches into the macrocycle cavity (Figure 14).

The alternating short-long pattern in the macrocycle bond lengths $\left(d_{\max }(C-C)=1.49 \AA\right.$, $d_{\text {min }}(C-C)=1.36 \AA$; cf. Chapter 6.1) is preserved upon oxidation. Therefore, the newly established pyrazoloindole moiety substructure does not connect the two separate conjugation pathways characteristic of Siamese-twin porphyrin $\mathbf{2 7} \mathbf{H}_{\mathbf{4}}$. However, the indole chromophore extends the $\pi$-conjugation of one of the two conjugated $\pi$-systems, thus

\footnotetext{
* The $d_{\text {helix }}$ represents the distance between the meso-carbon atoms of the dipyrrin moieties, $\tau$ represents the averaged angle between the planes defined by the two dipyrrine subunits, as illustrated in Figure 13D.
} 
rationalizing the bathochromic shift of the UV/vis spectrum of $\mathbf{3 2 H}_{3}$ compared with the spectrum of $\mathbf{2 7} \mathbf{H}_{\mathbf{4}}$ (Figure 11). The asymmetric structure seen in the solid state of mono-Nfused compound $\mathbf{3 2 H}_{3}$ is fully consistent with the NMR data presented below (Chapter 3.1.4).

The endo-cyclic location of the pyrazoloindole subunit with respect to the macrocyclic backbone in $\mathbf{3 2} \mathbf{H}_{3}$ (cf. idealized drawing in Scheme 8) lifts the complexation capacity of the previous $\left\{\mathrm{N}_{4}\right\}$-coordination pocket. The second, though highly distorted, coordination site still shows an inward-oriented preorganization of the donating units suitable for metal ion complexation. This option is explored further in Chapter 3.1.5.

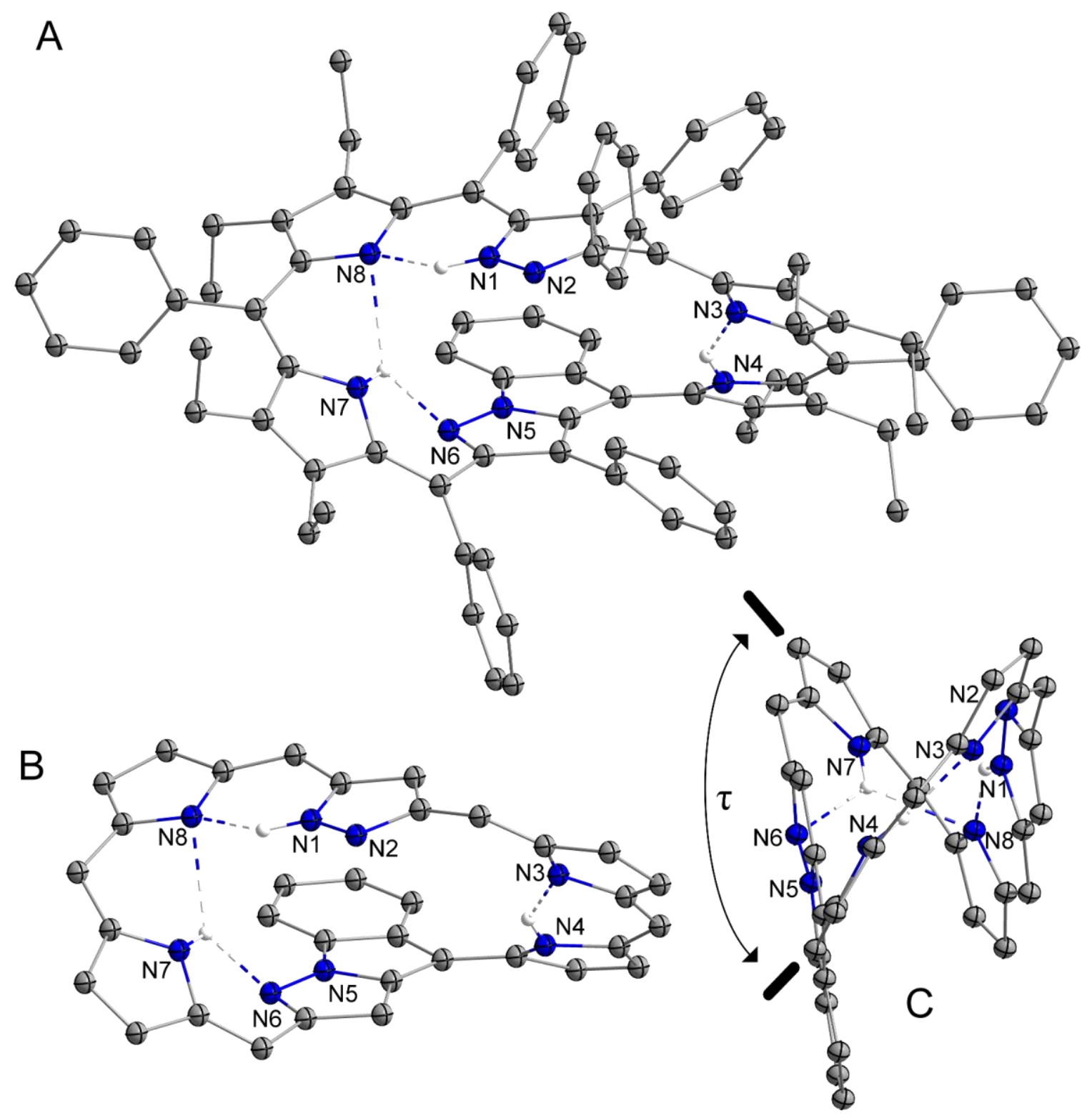

Figure 15: Ball-and-stick representation of the molecular structure of the mono-fused Siamese-twin porphyrin $\mathbf{3 2 H}_{3}$ in solid state (carbon in grey, nitrogen in blue and hydrogen in white; only one enantiomer shown; solvent molecules and carbon-bound hydrogen atoms omitted for clarity). A) Overview including peripheral substituents, B) core representation highlighting the tricyclic pyrazolo[1,5- $a$ ]indole unit (same view as in A) and C) view along the long axis depicting the torsion angle $\tau$. 

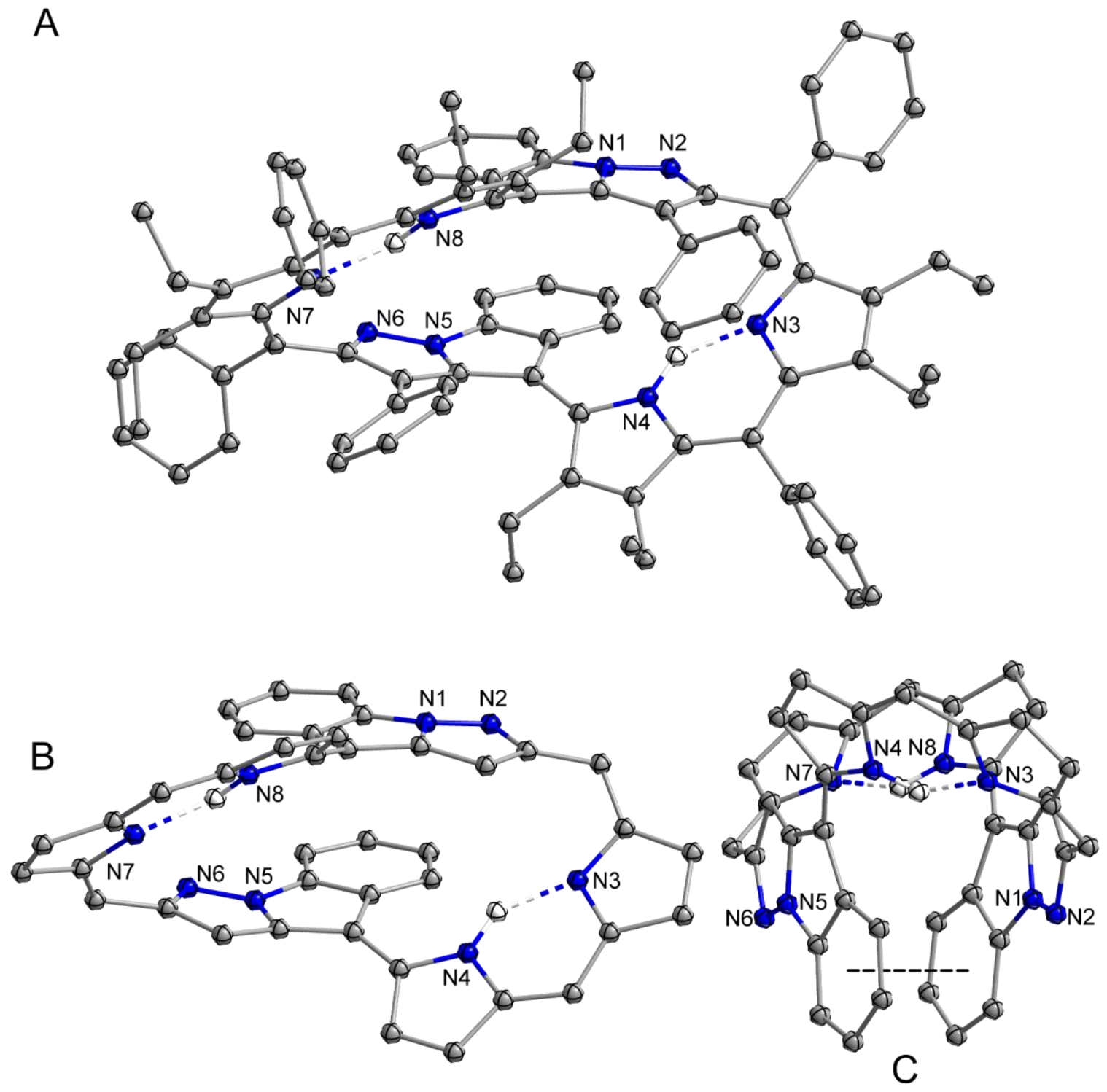

Figure 16: Ball-and-stick representation of the moleclar structure of the bis-fused Siamese-twin porphyrin $\mathbf{3 3 \mathbf { H } _ { 2 }}$ in solid state (carbon in grey, nitrogen in blue and hydrogen in white; only one enantiomer shown; solvent molecules and carbon-bound hydrogen atoms omitted for clarity). A) Overview including peripheral substituents, B) core representation (same view as in A), and C) representation of the $C_{2}$-symmetric core with view along the long axis highlighting the parallel arrangement of the two pyrazolo[1,5- $a$ ] indole moieties (indicated by a black dashed line).

Single crystals of the four-hydrogen oxidation product $\mathbf{3} 3 \mathbf{H}_{2}$ suitable for X-ray diffraction were obtained by layering a saturated solution of $\mathbf{3} 3 \mathbf{H}_{2}$ in $\mathrm{CH}_{2} \mathrm{Cl}_{2}$ with $\mathrm{MeOH}$. The diffraction data were resolved in the centrosymmetrical, monoclinic space group $P 2_{1} / n$ (Figure 16; Chapter 6.1). The macrocycle shows the presence of a second fusion of a meso-phenyl substituent with an inner pyrazole moiety. With a pyrazoloindole moiety present on each of the long sides, the pseudo- $C_{2}$-symmetry of the core is restored. The alternating short-long pattern in bond lengths $\left(d_{\max }(\mathrm{C}-\mathrm{C})=1.48 \AA, d_{\min }(\mathrm{C}-\mathrm{C})=1.37 \AA\right.$; cf. Chapter 6.1$)$ of the macrocycle is preserved. Hydrogen bonds between neighboring pyrrolic units lead to an alternating arrangement of planar building blocks linked by phenyl-substituted meso-carbon atoms, whereby the mean planes of the building blocks are arranged nearly perpendicular to 
each other.[94] Therefore, the overall shape of the molecule is a narrow, rectangular box, with the short side formed by the dipyrrin moieties and the long sides by the pyrazoloindole moieties (Figure 14, top view of $\mathbf{3 3} \mathbf{H}_{\mathbf{2}}$ ).

The structure of $\mathbf{3} 3 \mathbf{H}_{\mathbf{2}}$ is the result of a remarkable twist preceding the second pyrazole-tophenyl group fusion, presumably brought about by the steric constraints within the precursor macrocycle $\mathbf{3 2} \mathbf{H}_{3}$ (Figure 14). In $\mathbf{3 2} \mathbf{H}_{3}$, the pyrazole nitrogen atoms of the non-fused pyrazole point inward. Also, one of the two adjacent meso-phenyl groups is pointing inward. Therefore, the ortho-position is close to the pyrazole nitrogen atom $\left(d\left(\mathrm{~N} 2 \cdots \mathrm{Cortho}^{\mathrm{Ph}}\right)=3.30 \AA\right)$. In stark contrast to the first $\mathrm{N}$-fusion, however, this is not the phenyl group that fuses with the pyrazole nitrogen upon oxidation of $\mathbf{3 2} \mathbf{H}_{3}$. Instead, the macrocycle undergoes a remarkable eversion movement: the pyrazole flips about $180^{\circ}$ and fuses to the phenyl group located on the outside of the macrocycle. Next, the pyrazole phenyl group swings into the ring (compare color-coding in Figure 14). As a consequence of these twists and linkages, the two pyrazoloindole tricycles are arranged such that they are almost perfectly parallel $\left(\Varangle=3.8^{\circ}\right)$, with a short separation of their mean planes (3.6 ̊, Figure 16C), ${ }^{*}$ suggestive of the presence of $\pi$ - $\pi$-interactions. ${ }^{[94]}$ The pyrazoloindole units point to the same opening of the elongated box.

In contrast to examples of $\mathrm{N}$-linked hexaphyrins (such as 16,17 , or 19), in which the nitrogen atom of the fused pyrrole is presented on the outside of the macrocycle (or this conformer is the majority species), [55-57] the $\mathrm{N}$-linked products $\mathbf{3} \mathbf{2} \mathbf{H}_{\mathbf{3}}$ and $\mathbf{3} \mathbf{3} \mathbf{H}_{\mathbf{2}}$ introduced here present the fused polycyclic heterocycle structure on the inside of the macrocycle. This may be controlled by the $\beta$-carbon atoms of the inverted pyrroles, which correspond to the pyrazole nitrogen atoms in the Siamese-twin porphyrin backbone. It could also be related to the unique conformation of the backbone of the parent macrocycle $\mathbf{2 7} \mathbf{H}_{4}$ that already places a mesophenyl group in close proximity to the pyrazole moiety inside the macrocycle.

\subsubsection{NMR Spectroscopic Characterization}

A comprehensive NMR study on the behavior of Siamese-twin porphyrin $\mathbf{2 7} \mathbf{H}_{\mathbf{4}}$ in solution was previously presented by BLUSCH. ${ }^{[76]}$ An improved assignment of the spectra of $\mathbf{2 7} \mathbf{H}_{4}$ is given in detail in the Experimental Section. Thus, the following discussion will focus on the characteristic aspects of the spectra of $\mathbf{3 2} \mathbf{H}_{3}$ and $\mathbf{3} 3 \mathbf{H}_{2}$ that identify the presence and connectivity of the pyrazoloindole linkage products and that are diagnostic for the shape of the molecule in solution.

The ${ }^{1} \mathrm{H}$ NMR spectra of $\mathbf{2 7} \mathbf{H}_{\mathbf{4}}, \mathbf{3 2}_{3}$, and $\mathbf{3} 3 \mathbf{H}_{\mathbf{2}}$ (Figure 17) show three distinct groups of signals: two complex patterns with chemical shifts of $\delta=3.50-0.50$ and $8.00-6.50 \mathrm{ppm}$, and a singlet pattern at $\delta=16.00-10.00 \mathrm{ppm}$. Considering the macrocyclic structures, those patterns can be assigned to the aliphatic ethyl substituents, the aromatic phenyl substituents and the $\mathrm{NH}$ protons, respectively.

\footnotetext{
${ }^{*}$ Angles and distances were measured with the help of centroids of the six-membered ring.
} 


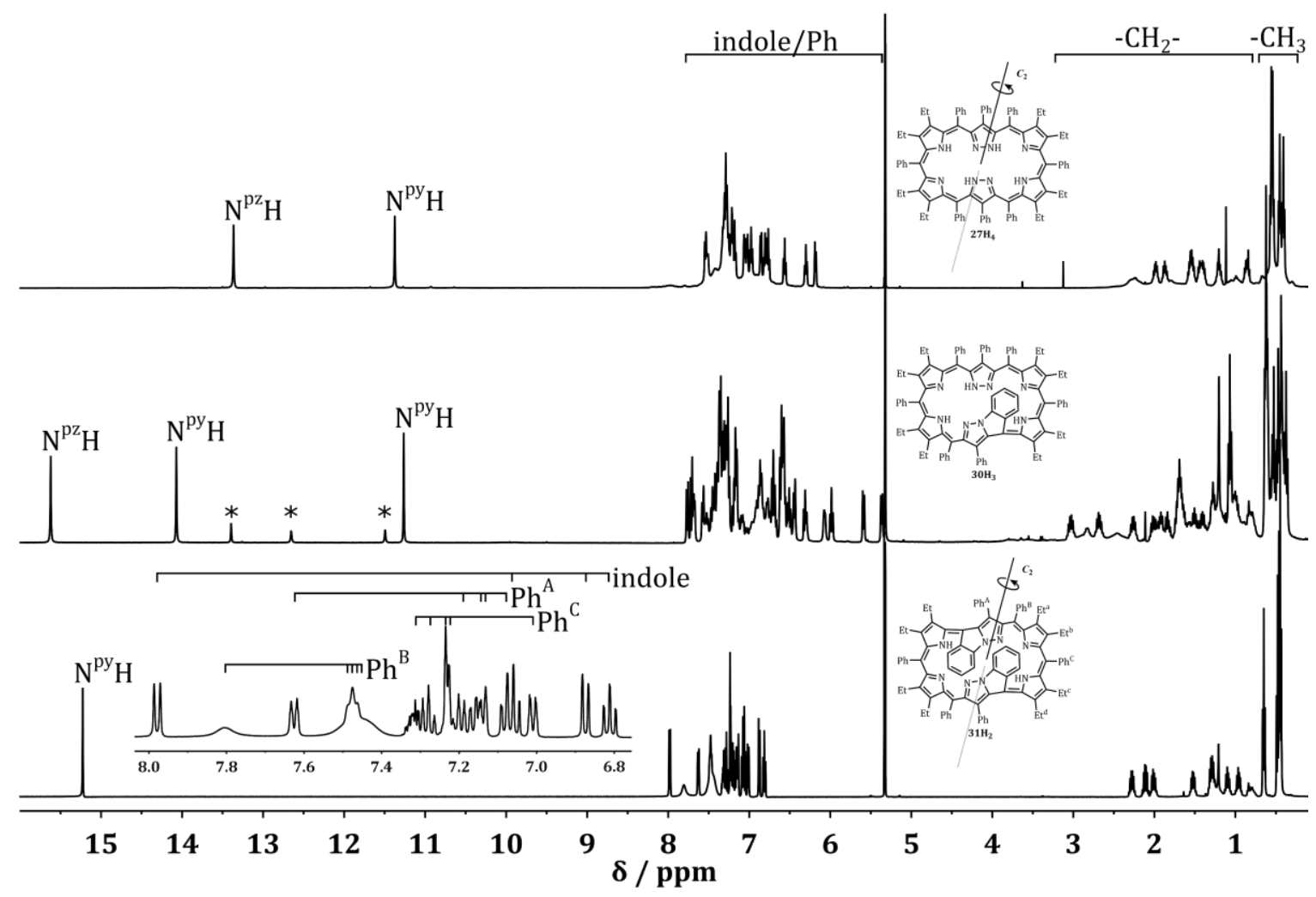

Figure 17: ${ }^{1} \mathrm{H}$ NMR spectra $\left(500 \mathrm{MHz}, \mathrm{CD}_{2} \mathrm{Cl}_{2}\right.$ ) of the Siamese-twin porphyrin $\mathbf{2 7 \mathbf { H } _ { 4 }}$ (at $238 \mathrm{~K}$ ), its two-hydrogen oxidation product $\mathbf{3 2 H}_{3}$ (at $243 \mathrm{~K}$ ) and its four-hydrogen oxidation product $\mathbf{3} 3 \mathbf{H}_{2}$ (at $243 \mathrm{~K}$ ). * The minor species detected in the spectrum of $\mathbf{3 2 H}_{3}$ at low temperatures were attributed to an isomer carrying a different protonation pattern.

The location of all NH signals in the deshielded region ( $\delta=16.00$ to $10.00 \mathrm{ppm}$ ) clearly points at the absence of any diatropic ring current that would be caused by a macrocycle aromatic $\pi$-system (Figure 17). Additionally, analysis of the $\mathrm{NH}$ region of the spectra shows the loss of two-fold symmetry upon oxidation of $\mathbf{2 7} \mathbf{H}_{4}$ to $\mathbf{3 2} \mathbf{H}_{3}$, and the recovery of the symmetry upon the second oxidation, forming $\mathbf{3} 3 \mathbf{H}_{2}$. In all of the spectra, fast proton exchange leads to broad signals at room temperature that sharpen upon cooling. The features seen in the NMR spectra of $\mathbf{3 2 H}_{3}$ are, as expected, largely a mixture between those found in the free-base parent macrocycle $\mathbf{2 7} \mathbf{H}_{4}$ and the bis-linked product $\mathbf{3 3} \mathbf{H}_{2}$. Therefore, spectra of $\mathbf{3 3} \mathbf{H}_{\mathbf{2}}$ will be discussed first.

The most obvious feature in the ${ }^{1} \mathrm{H}$ NMR spectrum of $\mathbf{3} 3 \mathbf{H}_{2}$ is its relative simplicity, reflecting the presence of a symmetric molecule. The number of peaks in the ${ }^{1} \mathrm{H}$ NMR and ${ }^{13} \mathrm{C} \mathrm{NMR}$ spectra suggest a two-fold symmetry axis. The absence of signals corresponding to $\mathrm{N}^{\mathrm{pz}} \mathrm{H}$ groups indicates that the oxidation reaction involved both pyrazole $\mathrm{NH}$ moieties.

A number of distinct spin systems can be found in the aromatic region. One particular set is observed at $\delta=7.98(\mathrm{~d}), 7.06(\mathrm{t}), 6.87(\mathrm{~d})$, and $6.81(\mathrm{t})$ ppm (Figure 18, green) corresponding to a meso-phenyl group, linked at the ortho-position.[95] These protons are all correlated to one another, confirmed by ${ }^{1} \mathrm{H},{ }^{1} \mathrm{H}-\mathrm{COSY}$ experiments (Figure S1) with a ${ }^{3} \mathrm{~J}$ coupling of $7.7 \mathrm{~Hz}$. The linkage is also consistent with ${ }^{1} \mathrm{H}^{15} \mathrm{~N}$ HMBC experiments, illustrating the proton at 
$\delta=6.87 \mathrm{ppm}$ couples to the pyrazole nitrogen atom at $\delta=152 \mathrm{ppm}$ (Figure S2). Thus, the results provide spectroscopic evidence for the oxidative formation of a $\mathrm{N}^{\mathrm{pz}}-\mathrm{Cortho}^{\text {-Ph }}$ linkage, overall forming a pyrazolo[1,5-a] indole moiety. Furthermore, three other correlated systems, with five proton resonances each, could be unambiguously identified and assigned to the remaining peripheral phenyl substituents (cf. color-coding in Figure 18).

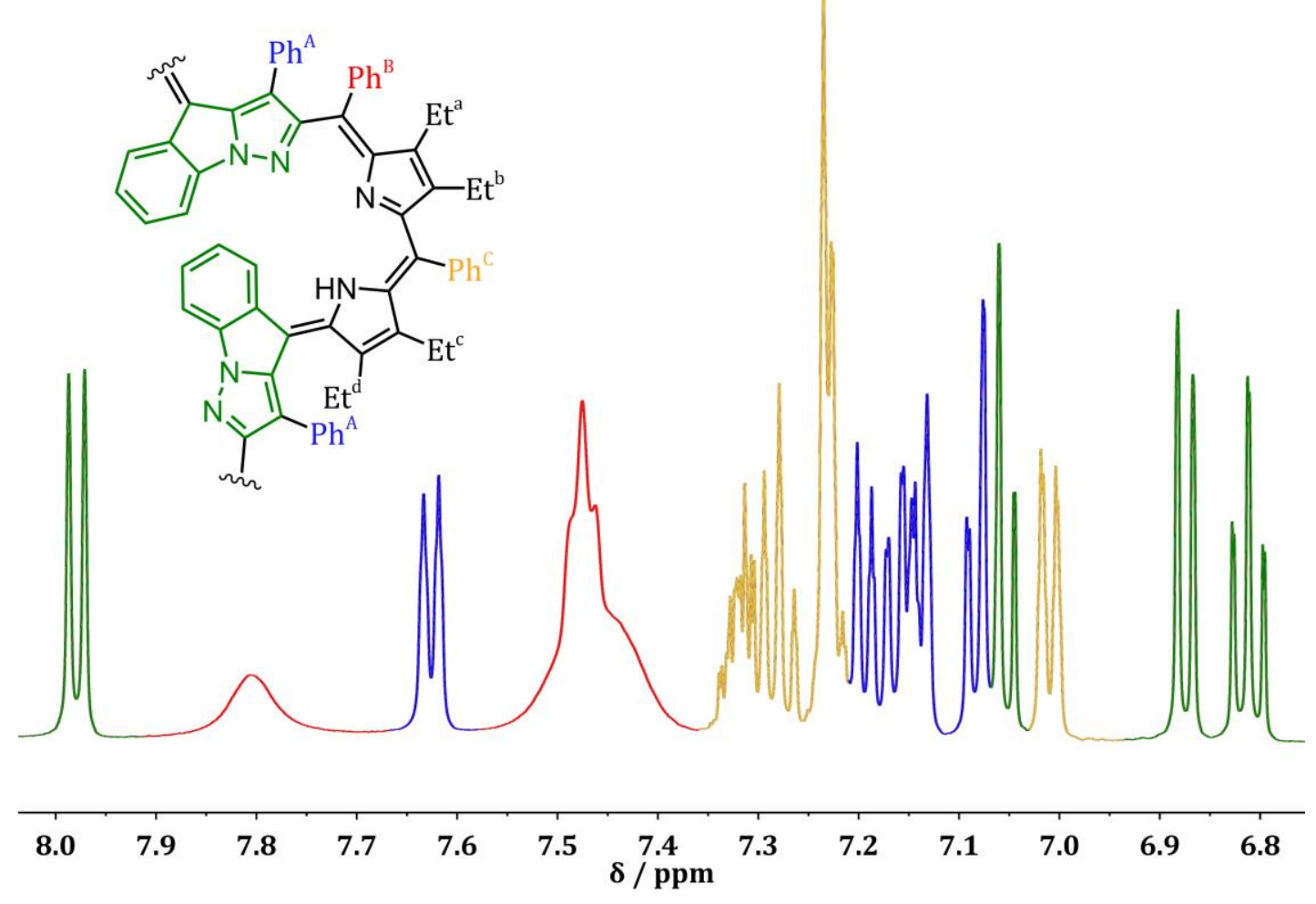

Figure 18: ${ }^{1} \mathrm{H}$ NMR spectrum $\left(500 \mathrm{MHz}, \mathrm{CD}_{2} \mathrm{Cl}_{2}, 243 \mathrm{~K}\right)$ of $\mathbf{3 3} \mathbf{H}_{2}$ between $\delta=8.1-6.7 \mathrm{ppm}$. Correlating signal sets are colored analogous to the section of ${ }^{1} \mathrm{H}^{1} \mathrm{H}-\mathrm{COSY}$ spectrum of $\mathbf{3 3} \mathbf{H}_{2}$ (Figure S1) and inset figure.

In fact, many other features of the bis-linked product $\mathbf{3} 3 \mathbf{H}_{\mathbf{2}}$ seen in the solid-state structure could also be confirmed spectroscopically, indicating that the solid-state structure is largely retained in solution. For instance, four spin systems belonging to ethyl groups in different environments and with diastereotopically split methylene groups could be distinguished (at $\delta=2.28,2.01$, and $0.65 \mathrm{ppm} ; \delta=2.11-0.95$ and $0.46 \mathrm{ppm}$; Figure S3). Their correlations were determined by ${ }^{1} \mathrm{H},{ }^{1} \mathrm{H}$-COSY experiments (Figure S4) and are consistent with the presence of a non-planar structure.

Furthermore, one set of methylene protons (Eta with $\delta=2.28,2.01$, and $0.65 \mathrm{ppm}$ ) possesses NOE cross peaks with the aromatic section of the spectrum, indicating it lies in close proximity to the meso-phenyl moiety $\mathrm{Ph}^{\mathrm{B}}$ (at $\delta=7.80,7.48$, and $7.46 \mathrm{ppm}$; encircled in grey in Figure 19), the phenyl group adjacent to the pyrazoloindole moiety. As can be seen in the ${ }^{1} \mathrm{H} N M R$, this phenyl group is also the least conformationally restricted, with signals coalescing as a result of a fast (at the ${ }^{1} \mathrm{H}$ NMR time scale) rotation around the meso-ipso axis (Figure S5). None of the peaks assigned to the indole portion of the pyrazoloindole moiety possess NOE cross peaks with any of the $\mathrm{CH} / \mathrm{CH}_{2}$ groups associated with the dipyrrin building blocks or mesophenyl groups (green-highlighted areas in Figure 19) indicating their spatial separation. 


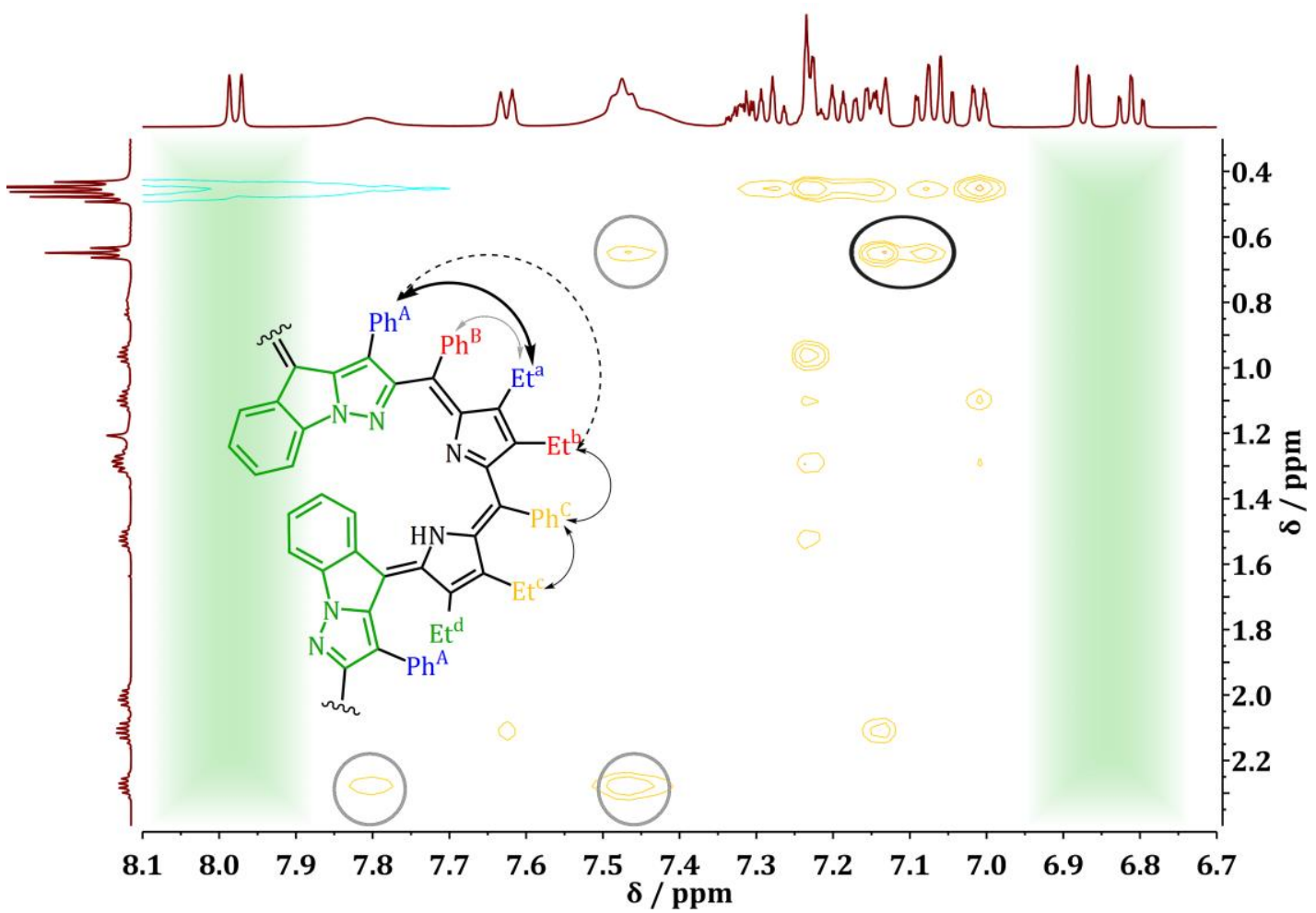

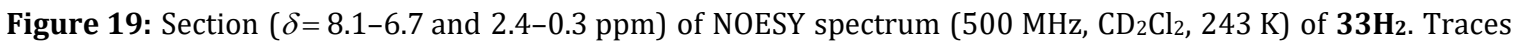
corresponding to the isolated pyrazoloindole substructure are highlighted in green. Cross peaks between the downfield-shifted methylene protons (Eta) and the freely rotating phenyl group $\mathrm{Ph}^{\mathrm{B}}$ are encircled in grey and between Eta and the phenyl group attached to the pyrazole $\mathrm{Ph}^{\mathrm{A}}$ are encircled in black.

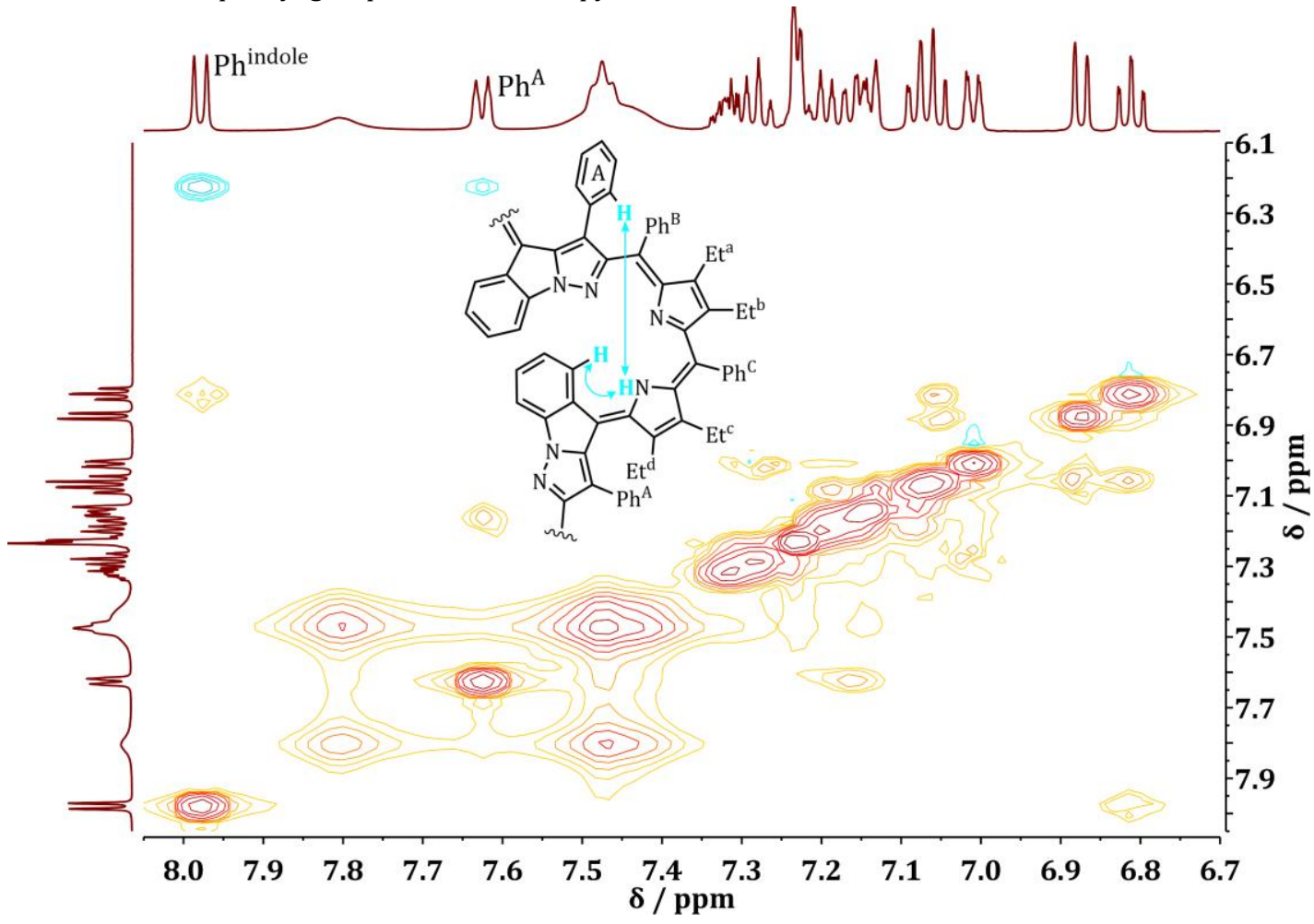

Figure 20: Aromatic section of the NOESY spectrum ( $500 \mathrm{MHz}, \mathrm{CD}_{2} \mathrm{Cl}_{2}, 243 \mathrm{~K}$ ) of $\mathbf{3 3} \mathbf{H}_{\mathbf{2}}$ with illustration of the origin for the cross peaks assigned to the NH protons (folded into the region depicted, in blue). Interactions between the pyrrolic $\mathrm{NH}$ proton and the pyrazoloindole ( $\left.\mathrm{Ph}^{\text {indole }}\right)$ as well as the pyrazoloindole bound phenyl group $\left(\mathrm{Ph}^{\mathrm{A}}\right)$ are shown with arrows. 
The aromatic section of the NOESY spectrum reveals two further remarkable features indicative of the preservation of the highly folded macrocyclic structure in solution: a strong NOE cross peak (Figure 20, in blue) between the indole ortho-CH proton ( $\delta=7.98 \mathrm{ppm})$ and the dipyrrin $\mathrm{NH}$ signal indicates their proximity, thus also illustrating spectroscopically the flip of the meso-phenyl upon fusion with the pyrazole. Another striking feature in the NOESY spectrum of $\mathbf{3} 3 \mathbf{H}_{2}$ (Figure 20, in blue) is diagnostic for the structure in solution: a cross peak between the dipyrrin $\mathrm{NH}$ and the ortho- $\mathrm{CH}\left(\mathrm{Ph}^{\mathrm{A}}\right)$ attached to the pyrazoloindole at the opposite side of the molecule - consequence of the folding of the macrocycle.

All spectral features described above are largely preserved over a temperature range of 298$243 \mathrm{~K}$, confirming the conformationally rather rigid structure of $\mathbf{3 3} \mathbf{H}_{\mathbf{2}}$. The high propensity of this derivative to form crystals may also suggest the presence of a relatively rigid molecule.

The features seen in the NMR spectra of $\mathbf{3 2} \mathbf{H}_{3}$ are, as expected, largely a mixture between those found in the free-base parent macrocycle $\mathbf{2} \mathbf{7 H}_{\mathbf{4}}$ and the bis-linked product $\mathbf{3} 3 \mathbf{H}_{\mathbf{2}}$. Although the NMR spectra are fairly complex, the majority of the signals of $\mathbf{3 2 H}_{3}$ could be assigned to substructures by using 1D and 2D techniques (Figure S6 to S8). Notable features include sixteen diastereotopic methylene protons and eight partially overlapping methyl groups, which can be distinguished (cf. ${ }^{1} \mathrm{H},{ }^{1} \mathrm{H}-\mathrm{COSY}$ in Figure S7), with one set (at $\delta=3.03$, 2.68, and $1.07 \mathrm{ppm}$ ) standing out. The significant diatropic downfield shift of this ethyl group relative to the signals of the parent compound $\mathbf{2 7} \mathbf{H}_{\mathbf{4}}$ can be attributed to the coplanar arrangement with the neighboring phenyl moiety $\mathrm{Ph}^{\text {a }}$ attached to the pyrazoloindole (Figure 14 and 15A). Again, this is an indication that the solid-state structure of this macrocycle also closely resembles the solution-state structure. The non-aromatic character of the molecule is reflected in the minor downfield shift of all $\mathrm{CH}$ protons of the endopositioned pyrazoloindole substructure. Notably, these phenyl- and indole-ethyl group interactions are lost as mono-linked $\mathbf{3 2} \mathbf{H}_{3}$ is oxidized to bis-linked $\mathbf{3} 3 \mathbf{H}_{\mathbf{2}}$ concomitantly undergoing a large macrocycle reorganization.

In conclusion, oxidation of the free-base Siamese-twin porphyrin $\mathbf{2 7 \mathbf { H } _ { 4 }}$ yielded two successive periphery-to-core linkages but did not lead to macrocycle aromatic products. The fusion involved a pyrazole nitrogen atom and an unactivated phenyl-CH group ( $\mathrm{N}^{\mathrm{pz}}-\mathrm{C}$ ortho-Ph fusion). This reaction stands in contrast to similar intramolecular ring fusions in expanded porphyrins (cf. Chapter 1.3.2).

\subsubsection{Complexation of Mono-Fused Siamese-Twin Porphyrin}

The highly distorted structure of the mono-fused Siamese-twin porphyrin $\mathbf{3 2 H}_{\mathbf{3}}$ presents a dibasic coordination site which appears suitable for the complexation of a single metal ion. As previously demonstrated for $\mathbf{2 7} \mathbf{H}_{4},{ }^{[82]}$ nickel(II) ions are readily incorporated under very mild reaction conditions and therefore represent an appropriate choice to verify the complexation capacity of the singly fused macrocycle $\mathbf{3 2} \mathbf{H}_{3}$.

Reaction of green, mono-fused Siamese-twin porphyrin $\mathbf{3 2} \mathbf{H}_{3}$ in a mixture of $\mathrm{MeOH} / \mathrm{CH}_{2} \mathrm{Cl}_{2}$ with $\mathrm{Ni}(\mathrm{OAc})_{2} \cdot 4 \mathrm{H}_{2} \mathrm{O}$ over two days at room temperature (Scheme 9) led to a subtle color change from green to yellow. TLC indicated the formation of a new, yellow-green product (34Ni) of higher polarity $\left(R_{\mathrm{f}}=0.40\right.$ vs $R_{\mathrm{f}}=0.54$ for $\mathbf{2 7 \mathbf { H } _ { 4 }}$; both neutral alumina, $n$-hexane/EtOAc 6:1) and a second high-polarity product (35HNi, $R_{\mathrm{f}}=0.00$; same conditions). 

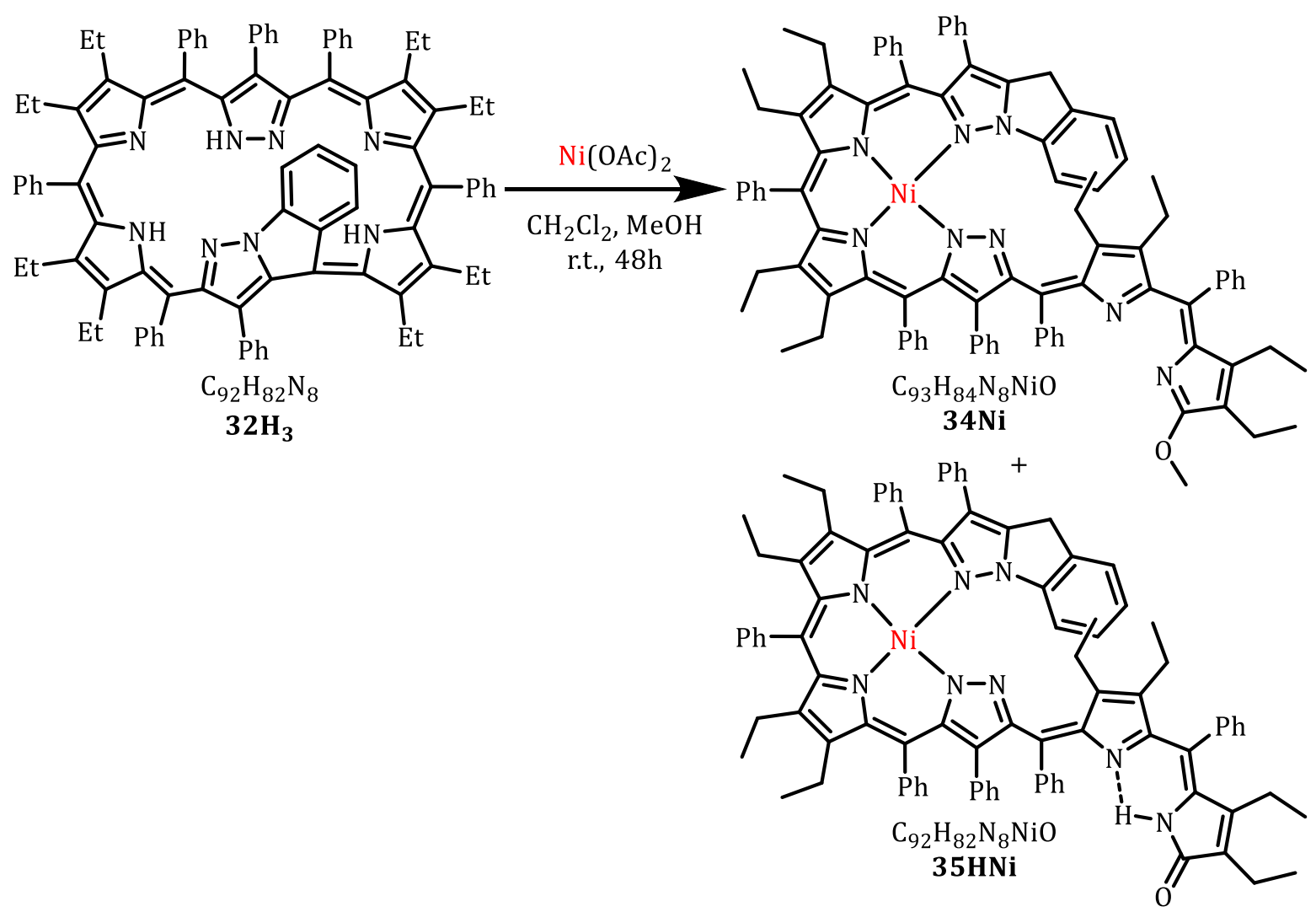

Scheme 9: Complexation of the mono-fused Siamese-twin porphyrin $\mathbf{3 2} \mathbf{H}_{3}$.

34Ni and 35HNi were isolated by column chromatography (neutral alumina, $n$-hexane/EtOAc 6:1 $\rightarrow$ pure EtOAc) as the first and second yellow-green fractions in $23 \%$ and $43 \%$ yields, respectively. The composition, as determined by HR-MS (ESI ${ }^{+}$), suggests that 34Ni is a nickel complex of a methanolysis product $\left(\mathrm{C}_{93} \mathrm{H}_{85} \mathrm{~N}_{8} \mathrm{NiO}^{+}\right.$for $\mathrm{MH}^{+}$; Figure 21) and $35 \mathrm{HNi}$ is a nickel complex of a hydrolysis product of $32 \mathrm{H}_{3}\left(\mathrm{C}_{92} \mathrm{H}_{83} \mathrm{~N}_{8} \mathrm{NiO}^{+}\right.$for $\mathrm{MH}^{+}$; Figure 22).
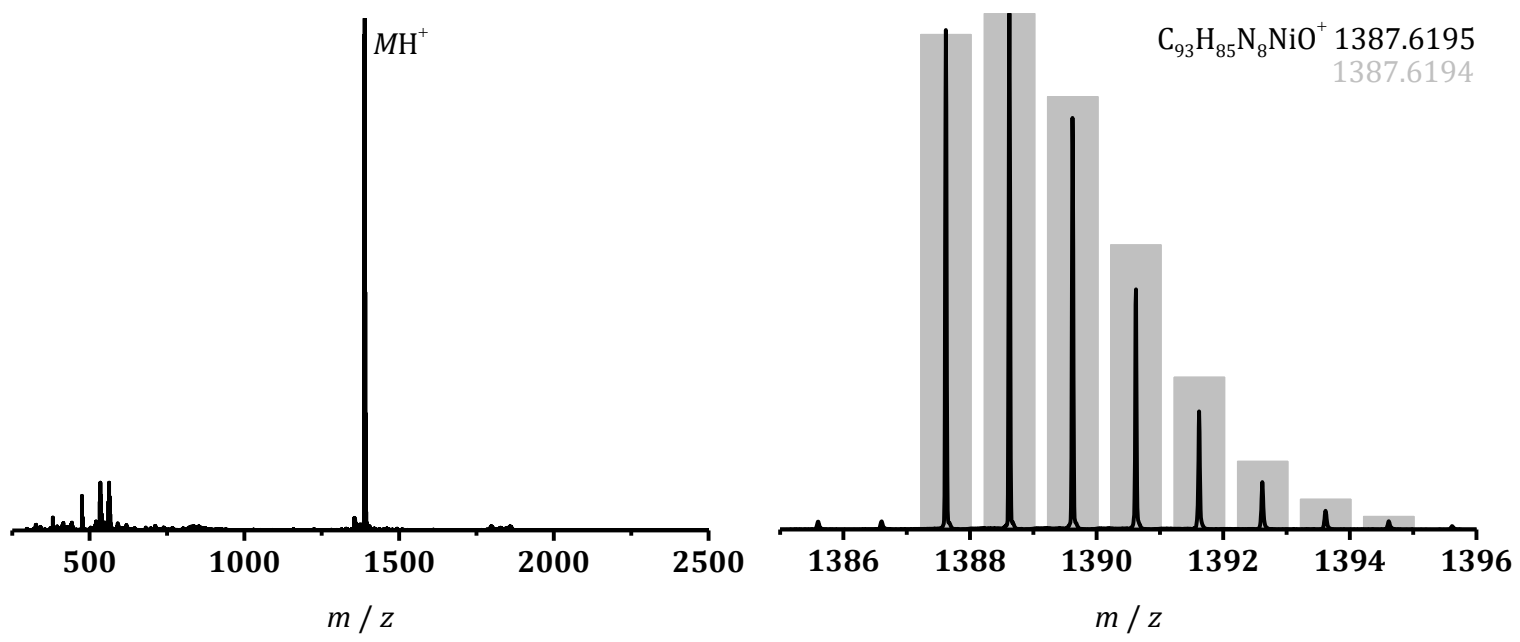

Figure 21: $\mathrm{HR}_{-\mathrm{ESI}^{+}}$spectrum of 34Ni and the isotopic distribution of the molecular peak $\mathrm{MH}^{+}$(simulation in grey). 

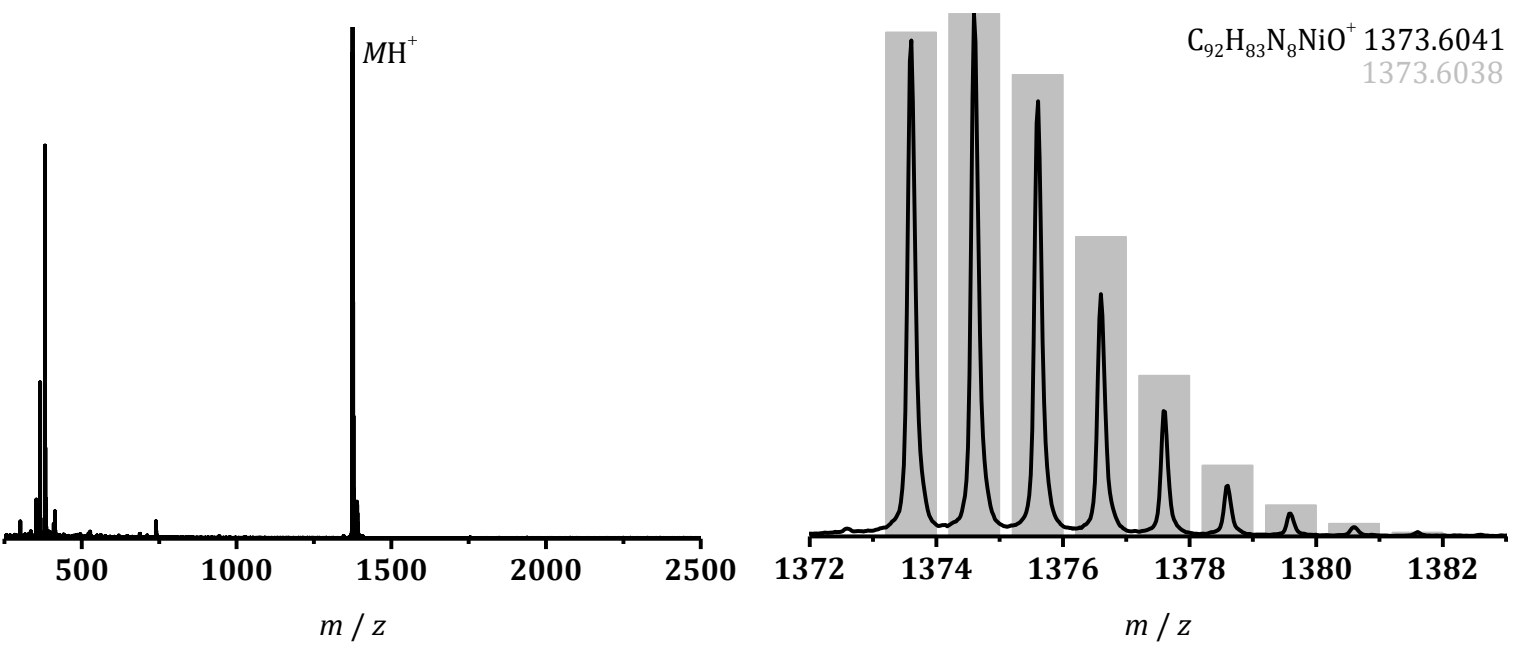

Figure 22: $\mathrm{HR}^{-\mathrm{ESI}^{+}}$spectrum of $\mathbf{3 5 H N i}$ and the isotopic distribution of the molecular peak $\mathrm{MH}^{+}$(simulation in grey).

The UV/vis spectral characterization of $\mathbf{3 4 N i}$ and $\mathbf{3 5 H N i}$ (Figure 23) shows significant loss in intensity $\left(\varepsilon<4.0 \times 10^{4} \mathrm{~L} \mathrm{~mol}^{-1} \mathrm{~cm}^{-1}\right)$ of all bands compared to the spectrum of the parent

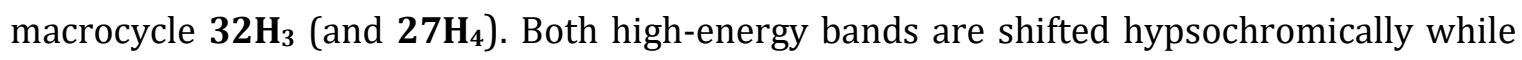
maintaining their overall shape. Since the main contribution are $\pi-\pi^{*}$-transitions, ${ }^{[83]}$ the overall $\pi$-system present in the parent molecule $\mathbf{3 2} \mathbf{H}_{3}$ is retained upon metalation. In contrast, the shape of the broad low-energy band changes dramatically. This observation is consistent with previous reports on metalation reactions of the Siamese-twin porphyrin $\mathbf{2 7} \mathbf{H}_{4}$. However, the band shape does not resemble the features seen in $\mathbf{3 2} \mathbf{H}_{3}$ or in $\mathbf{2} \mathbf{7} \mathbf{H}_{2} \mathbf{N i}$, indicating major alterations of the molecular structure in $34 \mathrm{Ni}$ and $35 \mathrm{HNi}$.
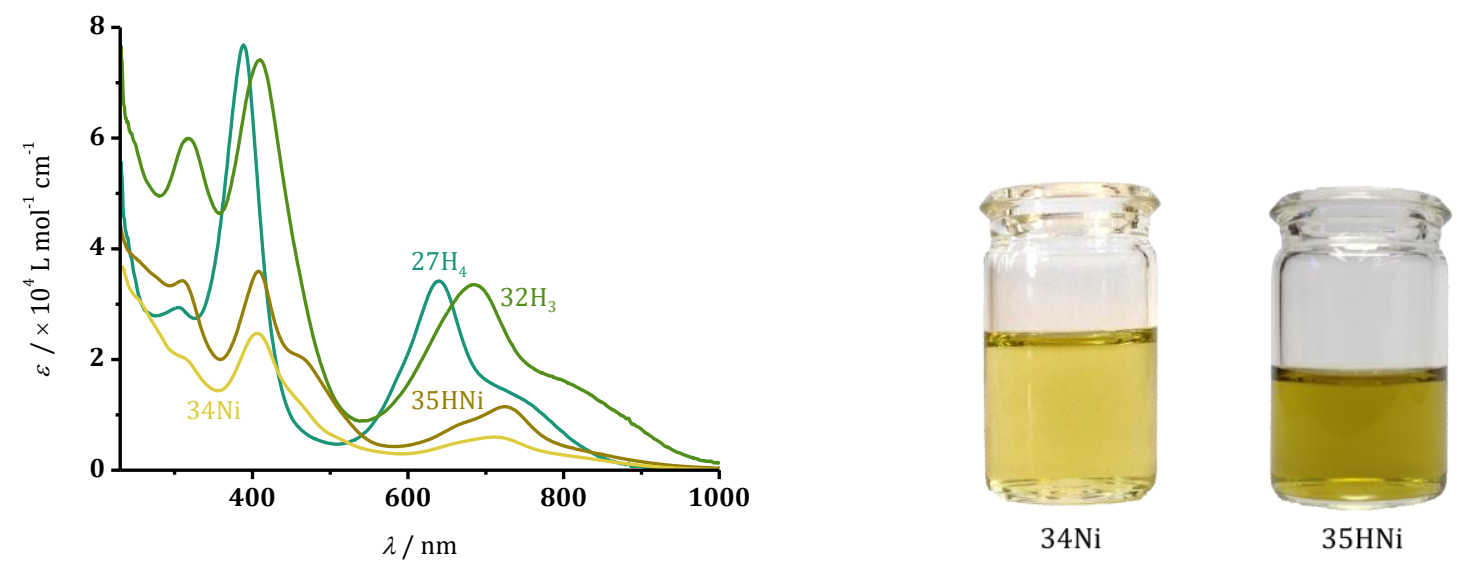

Figure 23: UV/vis spectra (298 $\mathrm{K}, \mathrm{CH}_{2} \mathrm{Cl}_{2}$ ) of Siamese-twin porphyrin $\mathbf{2 7} \mathbf{H}_{4}$ (teal trace), two-hydrogen oxidation product $32 \mathrm{H}_{3}$ (green trace), and the nickel complexes $34 \mathrm{Ni}$ and $35 \mathrm{HNi}$ (yellow traces).

Single crystals of hydrolyzed, mono-fused Siamese-twin porphyrin complex $35 \mathrm{HNi}$ suitable for X-ray diffration were obtained by layering of a saturated solution of $35 \mathrm{HNi}$ in $\mathrm{CH}_{2} \mathrm{Cl}_{2}$ with $n$-pentane. The data were resolved in the triclinic, non-chiral space group $P \overline{1}$, showing the presence of a racemic mixture of two helimeric conformers (Figure 24, Chapter 6.1). The overall shape of the molecule can be described as an oligo-heterocycle string arranged helically around a nickel(II) ion. The macrocyclic backbone of $\mathbf{3 2} \mathbf{H}_{3}$ is cleaved at the junction of the pyrazoloindole and the neighboring pyrrole units yielding a pyrrol-2-one on one end of 
the string and a methylene group of the pyrazoloindole moiety on the other. The pyrazoloindole-dipyrrin-pyrazole section of the molecule coordinates the nickel ion in a distorted square-planar fashion, while the remaining dipyrrin unit is twisted outwards thus releasing previous torsion strain (Figure 24A). The steric crowding around the metal center forces the pyrazoloindole subunit to be located above (or below) the molecular mean plane, consequently inducing molecular helicity (Figure 24B).

Due to the formation of both methanolysis and hydrolysis products, it may be assumed that the metalation-supported cleavage of the macrocycle proceeds via a nucleophilic attack of the

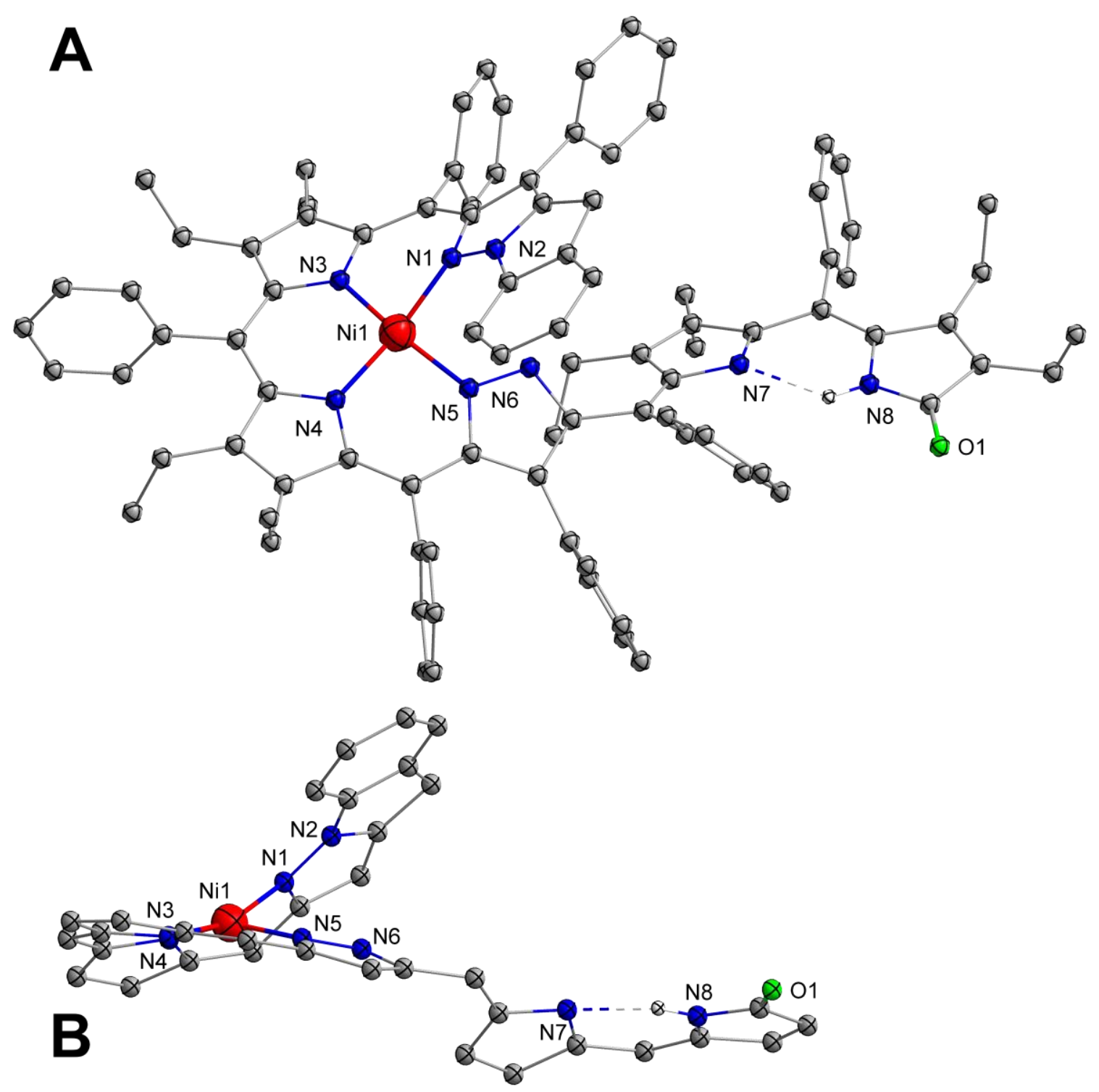

Figure 24: Ball-and-stick representation of the solid state structure of mono-fused Siamese-twin porphyrin complex 35HNi (nickel in red, carbon in grey, nitrogen in blue, oxygen in green and hydrogen in white). Only one enantiomer shown; solvent molecules and carbon-bound hydrogen atoms omitted for clarity. A) Overview including peripheral substituents; B) representation of macrocycle conformation highlighting the helical arrangement of the ligand. 
corresponding solvent molecule at the $\alpha$-carbon atom of the pyrrole. Formation of a hemiaminal (ether) followed by proton tautomerism may yield the structure of $\mathbf{3 5 H N i}$. However, this reaction pathway remains speculative since no reaction studies have been conducted.

Macrocyclic cleavage reactions are not uncommon within the group of metalated porphyrinoids. For instance, catabolism of chlorophylls involves the generation of oligopyrroles.[96] Furthermore, oxidative cleavage has also been reported for expanded porphyrins yielding unusual coiling oligopyrromethene helices with intense near-infrared (NIR) absorptions. [97] Similar to $35 \mathbf{H N i}$, the splitting occurs at a meso- $\alpha$-bond and the reaction is considered to be metal-supported. While the UV/vis region in the spectrum of $35 \mathrm{HNi}$ shows several absorptions with molar extinction coefficients comparable to those of coiling oligopyrromethene helices $\left(\varepsilon \approx 2.0-4.0 \times 10^{4} \mathrm{~L} \mathrm{~mol}^{-1} \mathrm{~cm}^{-1}\right)$, ${ }^{[97,98]}$ no intense NIR absorption could be detected.

Due to the low yield and limited amount of material, no further studies were conducted. Unfortunately, no single crystals for $\mathbf{3 4 N i}$ could be obtained. 


\subsection{Derivatization}

The favored formation of fused products over the expression of a macrocycle aromatic system upon oxidation of $\mathbf{2 7} \mathbf{H}_{4}$ underlines once again the key role of the pyrazole moiety in the interruption of an overall conjugation pathway in the conformationally strained Siamesetwin porphyrin $\mathbf{2 7} \mathbf{H}_{4}$. A modification of the electronic structure at this central unit appears beneficial for the connection of both isolated $\pi$-conjugated hemicycles. Additionally, steric crowding in the periphery along the long side of the molecule may be reduced by the choice of appropriate substituents.

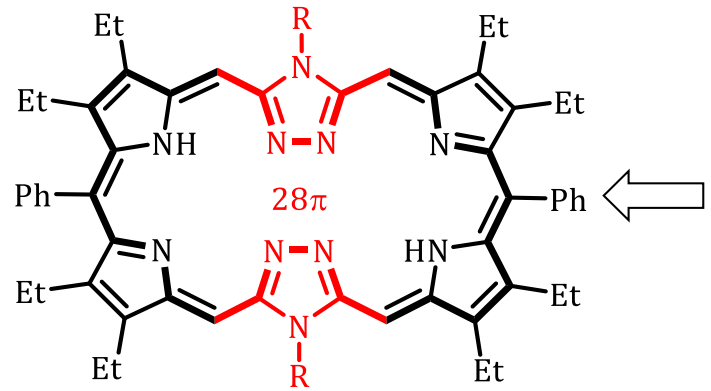

$\left(36 \mathrm{H}_{2}\right)$

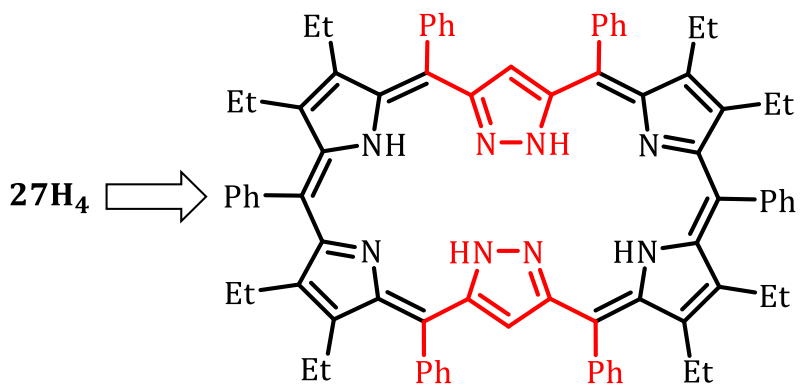

$\left(37 \mathrm{H}_{4}\right)$

Scheme 10: Targeted Siamese-twin porphyrin derivatives: On the left, triazole-substituted derivative $\mathbf{3 6 H}_{2}$ showing modified electronic structure at the merging points of both hemicycles and steric-bulk reduction at the adjacent meso-positions (highlighted in red; anticipated conjugation pathway highlighted in bold). On the right, derivative $\mathbf{3 7} \mathbf{H}_{\mathbf{4}}$ showing bulk-relieved substitution pattern on the pyrazole subunit (highlighted in red).

On the one hand, macrocycle $\mathbf{3 6} \mathbf{H}_{\mathbf{2}}$ (Scheme 10 ) was anticipated to possess a $28 \pi$-electron conjugation pathway. The altered electronic structure might not only serve as a connectivity link between the two hemicycles but also establish communication between both electronic subunits. On the other hand, target molecule $\mathbf{3 7} \mathbf{H}_{\mathbf{4}}$ was anticipated to show planarization of the macrocyclic twist due to release of steric strain upon removal of the central phenyl substitution. Additionally, the gain of rotational freedom for the pyrazole subunit may lead to new topological states suitable for the expression of macrocycle-wide aromaticity.

\subsubsection{Triazole Building Block}

For the derivatization of the macrocyclic core (towards $\mathbf{3 6} \mathbf{H}_{2}$ ) with direct impact on the electronic structure of the conjugated system, but not the connectivity, the triazole motif was chosen. This heterocycle shows high stability due to the aromatic character of its pentacyclic core. ${ }^{[99]}$ The lack of diene-character results in the majority of reactions occurring at the substituent groups. Furthermore, its amphoteric nature facilitates the isolation of products as precipitates.[99]

Chloro-substituted precursors were successfully applied in the synthesis towards Siamesetwin porphyrin $\mathbf{2 7}_{\mathbf{4}}$ by BLUSCH and syntheses of triazole-based ligands by BROOKER.[76,100] Thus, 3,5-bis(chloromethyl)-4-substituted-4H-1,2,4-triazole hydrochlorides $\mathbf{3 8} \cdot \mathbf{H C l}$ and 39.HCl) were chosen as starting materials (Scheme 11). The respective triazoles were obtained according to literature reported synthetic protocols.[101] The additional nitrogen atom of the triazole heterocycle in the 4-position is advantageous for a facilitated 

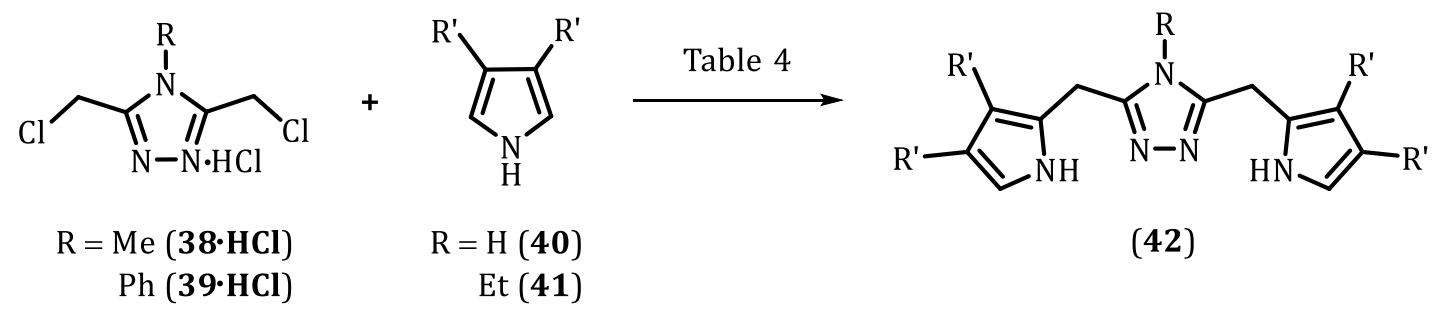

(42)

Scheme 11: Generalized reaction scheme towards a triazole/pyrrole-hybrid 42. Detailed synthetic strategies are summarized in Table 4.

derivatization of the backbone substituents and therefore control over the final macrocyclic twist in the Siamese-twin porphyrin derivative $\mathbf{3 6} \mathbf{H}_{2}$.

Initially, a synthesis strategy reported by BROOKER (strategy A, Table 4) was applied to link two pyrrole molecules (40 or $\mathbf{4 1}$ ) to the triazole hydrochloride $\mathbf{3 9} \cdot \mathbf{H C l}$. Hereby, an excess of a mild base $\left(\mathrm{K}_{2} \mathrm{CO}_{3}\right)$, catalytic amounts of $\mathrm{KI}$ and refluxing MeCN were used.[100] Unfortunately, only minor traces of a monosubstituted product could be detected by mass spectrometry from the crude reaction mixture. No quantitative conversion could be achieved and no isolation of Table 4: Summary of synthetic strategies towards a triazole/pyrrole-hybride building block $\mathbf{4 2}$.

\begin{tabular}{|c|c|c|c|c|c|}
\hline & triazole & pyrrole & solvent & additive & conditions \\
\hline \multirow{4}{*}{$\mathbf{A}$} & $39 \cdot \mathrm{HCl}$ & 41 & $\mathrm{MeCN}$ & $\begin{array}{l}\mathrm{K}_{2} \mathrm{CO}_{3}(10 \text { eq) } \\
\mathrm{KI}(0.1 \text { eq) }\end{array}$ & reflux, $21 \mathrm{~h}$ \\
\hline & $39 \cdot \mathrm{HCl}$ & 41 & THF (anhydr.) & $\begin{array}{l}\mathrm{K}_{2} \mathrm{CO}_{3}(10 \text { eq) } \\
\mathrm{KI}(0.1 \text { eq) }\end{array}$ & $\begin{array}{l}\text { r.t., } 21 \mathrm{~h} \\
\text { reflux, } 2 \mathrm{~d}\end{array}$ \\
\hline & $39 \cdot \mathrm{HCl}$ & 41 & MeCN (anhydr.) & $\begin{array}{l}\mathrm{K}_{2} \mathrm{CO}_{3}(10 \text { eq) } \\
\mathrm{KI}(0.1 \text { eq) }\end{array}$ & reflux, $2 \mathrm{~d}$ \\
\hline & 39 & 41 & MeCN (anhydr.) & $\mathrm{KI}(0.1 \mathrm{eq})$ & reflux, $2 \mathrm{~d}$ \\
\hline \multirow{3}{*}{ B } & 38 / 39 & 41 & MeCN (anhydr.) & $\mathrm{NaH}, i$-PrLi, MeLi & $-40^{\circ} \mathrm{C} \rightarrow$ r.t., $24 \mathrm{~h}$ \\
\hline & 39 & 41 & $\mathrm{CH}_{2} \mathrm{Cl}_{2}$ (anhydr.) & BuLi & $-78^{\circ} \mathrm{C} \rightarrow$ r.t., $24 \mathrm{~h}$ \\
\hline & 39 & 41 & toluene & DBU & reflux, $6 \mathrm{~h}$ \\
\hline C & 38 & 40 & none & $\mathrm{K}_{2} \mathrm{CO}_{3}$ & r.t. \\
\hline \multirow{2}{*}{ D } & & 41 & $\mathrm{CH}_{2} \mathrm{Cl}_{2}$ & $\begin{array}{l}\text { methanesulfonic } \\
\text { anhydride }\end{array}$ & reflux \\
\hline & $39 \cdot \mathrm{HCl}$ & 41 & $\mathrm{CH}_{2} \mathrm{Cl}_{2}$ & $\begin{array}{l}\text { methanesulfonic } \\
\text { anhydride } \\
\mathrm{Ag}_{2} \mathrm{CO}_{3}\end{array}$ & r.t., $2 \mathrm{~d}$ \\
\hline \multirow{3}{*}{$\mathbf{E}$} & $39 \cdot \mathrm{HCl}$ & 41 & $\mathrm{CH}_{2} \mathrm{Cl}_{2}$ (anhydr.) & $\mathrm{BF}_{3} \cdot \mathrm{OEt}_{2}$ & reflux $1 \mathrm{~d}$ \\
\hline & & 41 & $\mathrm{CH}_{2} \mathrm{Cl}_{2}$ & $\mathrm{BF}_{3} \cdot \mathrm{OEt}_{2}$ & reflux, $1 \mathrm{~d}$ \\
\hline & $39 \cdot \mathrm{HCl}$ & 41 & pyridine & $\mathrm{Ag}_{2} \mathrm{CO}_{3}$ & r.t., $31 / 2 \mathrm{~h}$ \\
\hline
\end{tabular}


the monosubstituted product was possible. Variation of reaction conditions did not lead to any improvements regarding yield or formation of a disubstituted product $\mathbf{4 2}$.

Consequently, the strategy was altered towards the use of stronger bases with differing steric bulk (strategy B, Table 4). Deprotonation of the pyrrole $\mathbf{4 1}$ according to established protocols ${ }^{[102]}$ and subsequent addition of free-base triazole 39 did not lead to formation of the desired hybrid 42. Quenching of the reaction mixtures with aqueous $\mathrm{NH}_{4} \mathrm{Cl}$ solution yielded hydrolysis products of the starting materials $\mathbf{3 8}$ and $\mathbf{3 9}$, respectively. Thus, the triazole building block appears in general susceptible to a nucleophilic attack, but so far reluctant to a coupling reaction with 3,4-diethylpyrrole (41).

Extreme excess of a sterically less crowded, unsubstituted pyrrole $\mathbf{4 0}$ (used as solvent; strategy C) did not lead to any product formation either. Substitution of chloride for a better leaving group (mesylate ester; strategy D) led to the formation of minimal amounts of a monosubstituted triazole/pyrrole hybrid. All attempts to increase the yield failed. Finally, LEWIS acids were used to facilitate abstraction of the chloride leaving group supporting the nucleophilic attack at the carbon atom (strategy E). However, no reaction could be traced.

In conclusion, synthesis of a triazole/pyrrole-hybrid $\mathbf{4 2}$ as a key intermediate analogous to the pyrazole/pyrrole-hybrid $\mathbf{2 9}$ was unsuccessful. Different synthetic protocols targeting the activation of either the pyrrole or the triazole compounds were applied. Unfortunately, no conversion to the desired product could be detected. Thus, this derivatization project towards triazole-substituted Siamese-twin porphyrin $\mathbf{3 6} \mathbf{H}_{2}$ was abandoned for the benefit of more detailed studies on oxidation and metalation of the Siamese-twin porphyrin $\mathbf{2 7} \mathbf{H}_{\mathbf{4}}$.

\subsubsection{Pyrazole Building Block}

Steric crowding on the long side of the Siamese-twin porphyrin $\mathbf{2 7 \mathbf { H } _ { 4 }}$ was shown to be accountable for the expression of the macrocyclic twist. ${ }^{[76]}$ Additionally, topology and spatial arrangement releasing steric tension among the peripheral substituents was shown to influence the reactivity of the macrocycle upon oxidation (Chapter 3.1). Thus, considering the key building blocks $\mathbf{2 8}$ and $\mathbf{2 9}$ (Scheme 5), synthesis of a 4-unsubstituted pyrazole derivative $\mathbf{4 6}$ as a key intermediate towards $\mathbf{3 7 \mathbf { H } _ { 4 }}$ (Scheme 10) was targeted (Scheme 12).

Retro-synthetically, 3,5-dibenzyl-1H-pyrazole (46) can be prepared by a condensation reaction of 1,5-diphenylpentane-2,4-dione (45) and hydrazine. The symmetric $\beta$-diketone, in turn, is available through a CLAISEN condensation of 1-phenylpropan-2-one (43) and ethyl<smiles>CC(=O)Cc1ccccc1</smiles>

(43)<smiles>CCOC(=O)Cc1ccccc1</smiles>

(44)

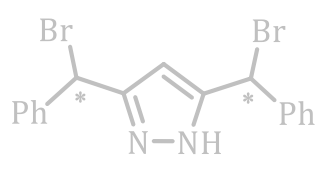

(4.7)
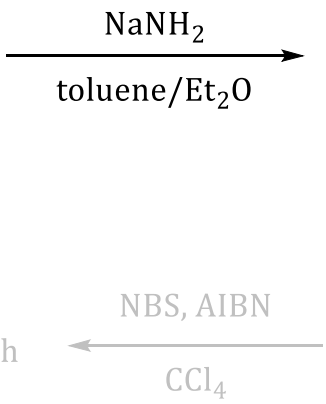<smiles>N#Cc1c(Cc2ccccc2)n[nH]c1Cc1ccccc1</smiles><smiles>O=C(CC(=O)Cc1ccccc1)Cc1ccccc1</smiles>

(45)

(46)

Scheme 12: Synthesis strategy towards a 3,5-dibenzyl-1 $H$-pyrazole building block 46. 
2-phenylacetate (44). Although both starting materials are inexpensive, availability of 1-phenylpropan-2-one (43) is limiting to the practicality of this study. Due to the illicit uses in clandestine chemistry towards methamphetamines, $\mathbf{4 3}$ is listed among controlled substances (category 1) and requires special permission for ordering or synthesis and synthetic derivatization.[103] Its precursor (phenylacetic acid) is covered by the same restrictive regulations and as a consequence does not present a suitable alternative.[103]

For proof of principle, synthesis towards $\mathbf{4 6}$ was carried out on a small scale. Condensation of ketone $\mathbf{4 3}$ and ester $\mathbf{4 4}$ yielded a mixture of products due to the presence of acidic protons in the electrophilic component and thus formation of various potential enoles from both reactants. ${ }^{[104]}$ Both major products (45 and 48, Scheme 13) were isolated as a mixture. Contrary to the reported literature procedure, ${ }^{[104]}$ no further separation could be achieved. Alternative clean-up procedures involving column chromatography, bulk-to-bulk distillation and repeated recrystallization failed due to the closely similar properties for $\mathbf{4 5}$ and $\mathbf{4 8}$.

Anticipating a potential change in properties and a concomitant facilitated separation of products, the mixture of 1,5-diphenylpentane-2,4-one (45) and ethyl 3-oxo-2,4-diphenylbutanoate (48) was used for a subsequent condensation reaction with hydrazine hydrate to yield 3,5-disubstituted pyrazole 46 and the byproduct 49 . While the reaction proceeded smoothly, separation of products ( 46 and 49 ) remained challenging.<smiles>CC(=O)Cc1ccccc1</smiles>

(43)

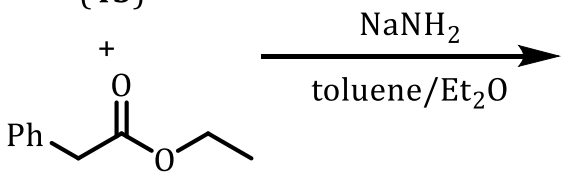

(44)<smiles>O=C(CC(=O)Cc1ccccc1)Cc1ccccc1</smiles>

(45)

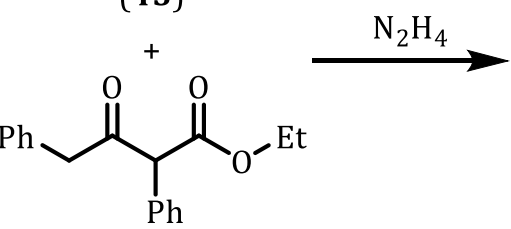

(48)

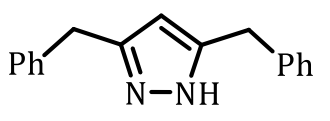

(46)

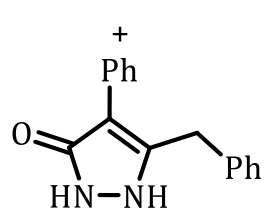

(49)

Scheme 13: Product mixtures observed during the condensation of 1-phenylpropan-2-one (43) and ethyl 2-phenylacetate (44).

Despite continuous separation efforts, the ${ }^{1} \mathrm{H}$ NMR spectrum (Figure 25) showed the presence of both products in a nearly 1:1 ratio. A complex multiplet with $\delta=7.5-6.9 \mathrm{ppm}$ was assigned to overlapping resonances of all four phenyl substituents present in 46 and 49 . Resonances at $\delta=5.72$ and $3.84 \mathrm{ppm}$ (Figure 25, blue), with integrals for one and four protons, corresponded to the proton in 4-position and methylene groups of the pyrazole 46, respectively. A singlet at $\delta=4.01 \mathrm{ppm}$ (Figure 25, red) was assigned to the single methylene group in 49. A very broad signal ( $\delta=11.68 \mathrm{ppm}$ ) indicated the presence of NH-group, but did not allow accurate integration. No signals indicating a lactam-lactim tautomerization were observed for $\mathbf{4 9}$.

Furthermore, single crystals suitable for X-ray diffraction were obtained by slow evaporation of a saturated solution of the mixture of $\mathbf{4 6}$ and $\mathbf{4 9}$ in EtOH. Resolved in the monoclinic, nonchiral space group $P 2{ }_{1} / c$ (Figure 26; Chapter 6.1), the structure revealed a co-crystallization of 3,5-dibenzyl-1H-pyrazole (46) and 5-benzyl-4-phenyl-1,2-dihydro-3H-pyrazol-3-one (49) in a $1: 1$ ratio. 
$\overbrace{\mathrm{N}-\mathrm{NH}}^{\mathrm{Ph}}$

(46)<smiles>O=c1[nH][nH]c(Cc2ccccc2)c1-c1ccccc1</smiles>

(49)

12

11

10

$\boldsymbol{\delta} / \mathbf{p p m}$

Figure 25: ${ }^{1} \mathrm{H}$ NMR spectrum (300 MHz, DMSO-d6, $301 \mathrm{~K}$ ) of product mixture of 46 and 49. Unambiguously assigned signals are colored according to given structures ( 46 in blue, 49 in red).

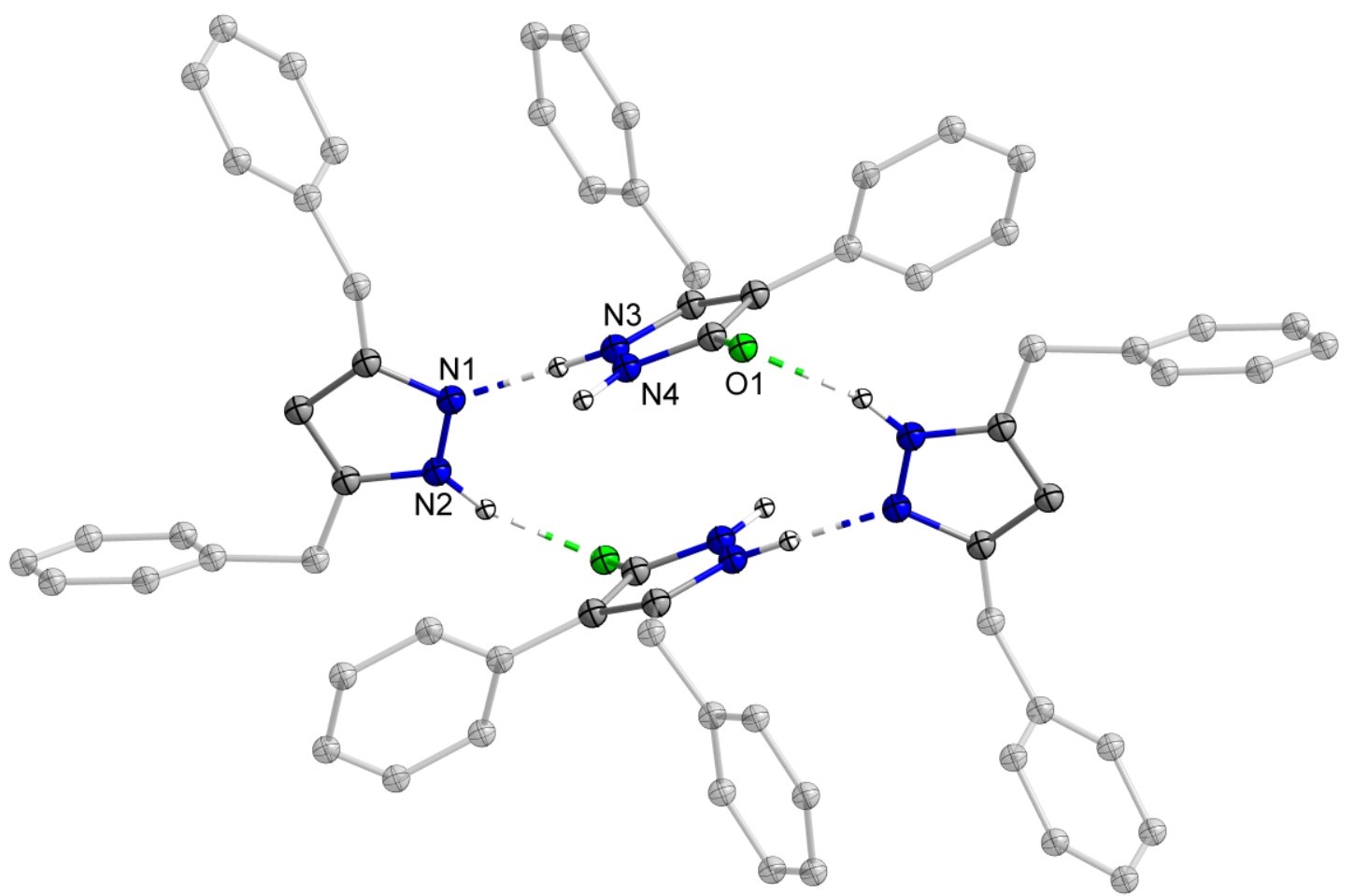

Figure 26: Ball-and-stick representation of the solid state structure of co-crystalized 3,5-dibenzyl-1H-pyrazole (46) and 5-benzyl-4-phenyl-1,2-dihydro-3 H-pyrazol-3-one (49) (carbon in grey, nitrogen in blue, oxygen in green and hydrogen in white). Periphery is faded out and carbon-bound hydrogen atoms are omitted for clarity; view along $C_{2}$-symmetry axis. 
Strong hydrogen bonding locks the heterocycles in a [2×2]-tetramer with macroscopic $C_{2}$-symmetry. Pyrazolones 49 arranged in a parallel fashion with respect to each other and bracketed by orthogonally positioned pyrazoles $\mathbf{4 6}$ via hydrogen bonds, resulting in a mean distance of $d(\mathbf{4 9 - 4 9 )}=3.4 \AA$, suggestive of $\pi$ - $\pi$-interactions.* The formation of such clusters accounts well for the described complications during separation.

Despite the simplicity and symmetry of the targeted pyrazole derivative 46 , synthesis and in particular isolation of the product proved to be challenging. As proof of principle, synthesis of sterically unburdened pyrazole $\mathbf{4 6}$ is feasible, but associated with severe obstacles. Thus, also this derivatization project towards sterically less crowded Siamese-twin porphyrin $\mathbf{3 7 H}_{4}$ was abandoned for the benefit of more detailed studies on oxidation and metalation of the Siamese-twin porphyrin $\mathbf{2 7} \mathbf{H}_{\mathbf{4}}$.

* Distance was measured with the help of centroids of the five-membered ring. 


\subsection{Manganese Complexes}

Following the studies on the redox properties and concomitant changes in the macrocyclic scaffold of the Siamese-twin porphyrin $\mathbf{2 7} \mathbf{H}_{4}$, the focus was shifted towards the investigation of interactions between the redox non-innocent ligand and redox active metal ions. In particular, encouraged by reports on high-valent manganese porphyrin complexes (cf. Chapter 1.2),[105] manganese complexes were targeted.

Initially, established synthesis strategies were used to test the metalation behavior and formation of suitable precursors, and subsequently experiments towards formation of highvalent species were performed. Parts of the following chapters (complexation with $\mathrm{Mn}(\mathrm{OAc})_{2}$ and analyses) were published before as a master thesis[106] and are repeatedly mentioned here for a complete overview and more comprehensive comparison with new findings.

\subsubsection{Siamese-Twin Porphyrin Metalation}

Metalation of the Siamese-twin porphyrin $\mathbf{2 7} \mathbf{H}_{4}$ with manganese salts was carried out in close analogy to the procedure reported by BLUSCH for the corresponding copper and nickel complexes.[75] $\mathrm{Mn}(\mathrm{OAc})_{2}$ served as the reagent of choice due to the combination of the metal cation in an appropriate oxidation state and the anion providing satisfactory solubility in the selected solvent and serving as a base for released protons during the reaction.

Reaction of blue-green Siamese-twin porphyrin $\mathbf{2 7}_{\mathbf{4}}$ with four equivalents of $\mathrm{Mn}(\mathrm{OAc})_{2}$ under inert conditions over two days at room temperature in methanol (Scheme 14) led to an

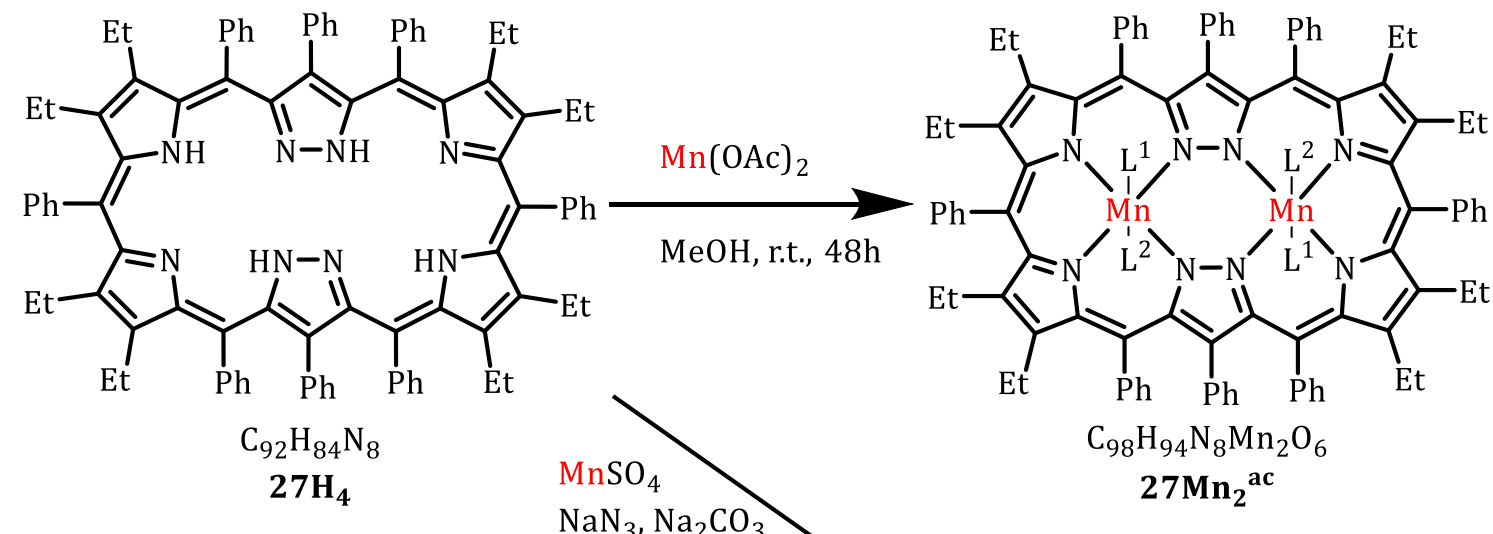

$\mathrm{MeOH}$, r.t., $24 \mathrm{~h}$

$\mathrm{L}^{1}=\mathrm{MeOH}$

$\mathrm{L}^{2}=\mathrm{AcO}^{-}$

$\mathrm{L}^{3}=\mathrm{N}_{3}^{-}$

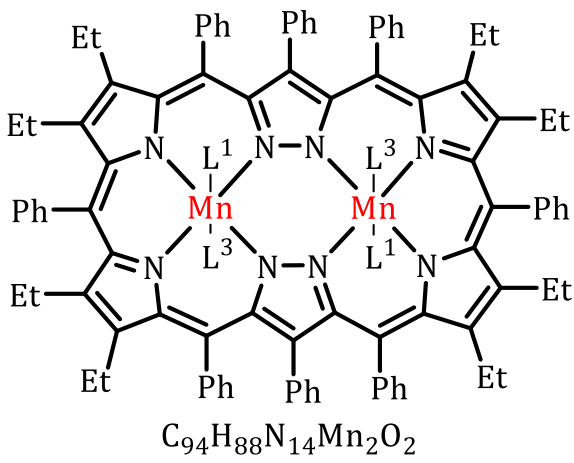

$27 \mathrm{Mn}_{2}{ }^{\text {az }}$

Scheme 14: Synthesis of dimanganese complexes $\mathbf{2 7} \mathbf{M n}_{2}{ }^{\text {ac }}$ and $\mathbf{2 7} \mathbf{M n}_{2^{a z}}$. 
olive-green color of the reaction mixture. TLC indicated the consumption of the free-base ligand $\mathbf{2 7} \mathbf{H}_{4}$ and mass spectrometry indicated the formation of a single new product $\left(\mathbf{2 7} \mathbf{M n}_{2}{ }^{\text {ac}}\right)$. Product $\mathbf{2 7} \mathbf{M n}_{2}{ }^{\text {ac }}$ was isolated in $40 \%$ yield by precipitation from methanol. The composition, as determined by $\mathrm{HR}-\mathrm{MS}\left(\mathrm{ESI}^{+}\right)$, suggested that $\mathbf{2 7} \mathbf{M n}_{\mathbf{2}} \mathbf{a c}^{\mathrm{ac}}$ is a dimanganese complex of $\mathbf{2 7} \mathbf{H}_{4}\left(\mathrm{C}_{94} \mathrm{H}_{83} \mathrm{~N}_{8} \mathrm{Mn}_{2} \mathrm{O}_{2}{ }^{+}\right.$for $M^{+}$; Figure 27).
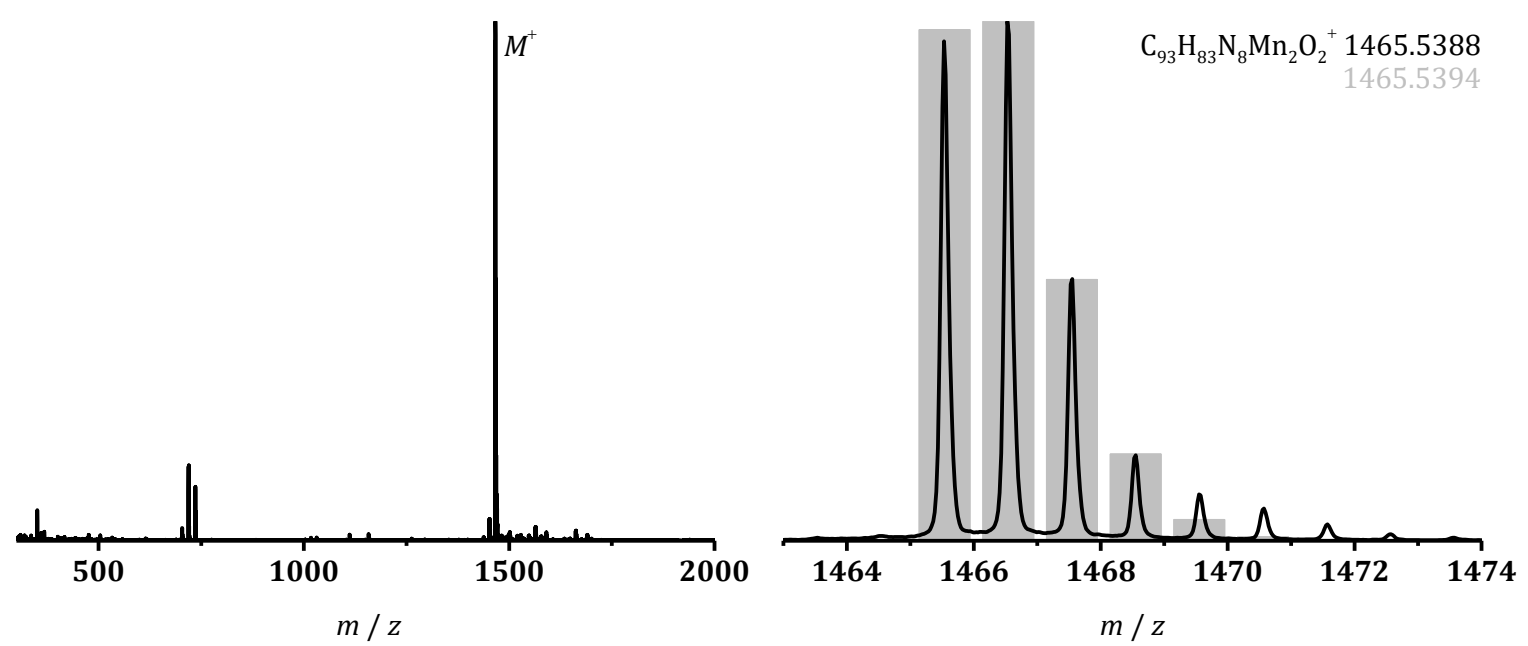

Figure 27: $\mathrm{HR}_{-}-\mathrm{ESI}^{+}$spectrum of $\mathbf{2 7} \mathbf{M n}_{2}$ ac and the isotopic distribution of the molecular peak $M^{+}$(simulation in grey).

The synthetic conditions could be optimized to a reaction in methanol at $50^{\circ} \mathrm{C}$ over $30 \mathrm{~min}$ with similar yields. Complexation reactions under acetate-free conditions $\left(\mathrm{MnCl}_{2}, \mathrm{MnSO}_{4}\right.$, $\left.\mathrm{MnCO}_{3}\right)$ or in other solvents $\left(\mathrm{CH}_{2} \mathrm{Cl}_{2}\right.$, THF, benzene, MeCN) proved to be ineffective. Due to the lack of poorly soluble acetate salts, complex $\mathbf{2 7} \mathbf{M n}_{2} \mathbf{a}^{\text {ac }}$ did not serve as a suitable precursor for axial ligand exchange via salt metathesis. Thus, direct synthesis towards $\mathbf{2 7} \mathbf{M n}_{\mathbf{2}} \mathbf{x}$ was required. Solely one reaction using $\mathrm{MnSO}_{4}$ as a manganese(II) source with a non-coordinating anion, $\mathrm{NaN}_{3}$ as a source for a slim, strongly coordinating axial ligand and $\mathrm{Na}_{2} \mathrm{CO}_{3}$ as a noncoordinating and easily removable base proved to be a viable alternative.
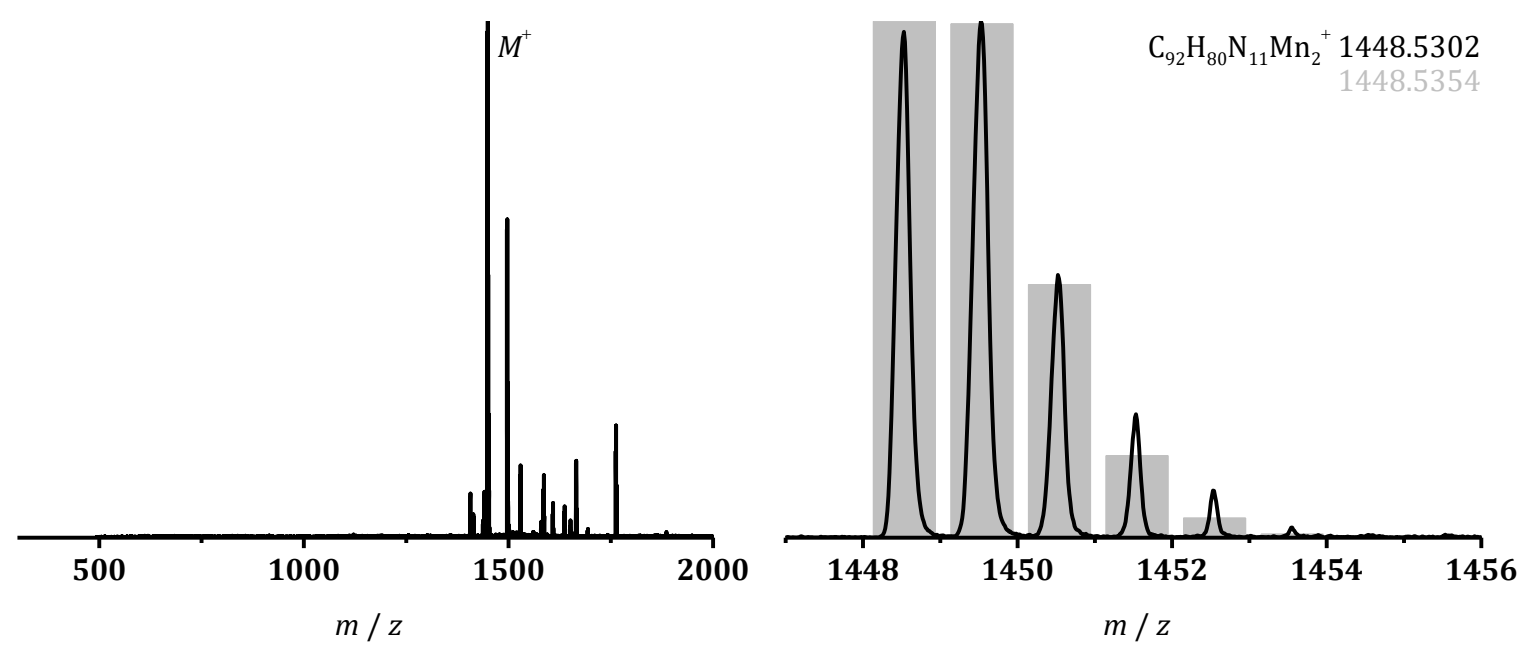

Figure 28: HR-MALDI spectrum of $\mathbf{2 7} \mathbf{M n} \mathbf{n}^{\text {az }}$ and the isotopic distribution of the molecular peak $M^{+}$(simulation in grey). 
Based on these findings, reaction of blue-green Siamese-twin porphyrin $\mathbf{2 7} \mathbf{H}_{4}$ with twenty equivalents of $\mathrm{MnSO}_{4}, \mathrm{NaN}_{3}$ and $\mathrm{Na}_{2} \mathrm{CO}_{3}$ under inert conditions overnight at room temperature in methanol (Scheme 14) led to a red-colored reaction solution and a dark-green precipitate. TLC indicated the consumption of the free-base ligand $\mathbf{2 7} \mathbf{H}_{\mathbf{4}}$ and mass spectrometry indicated the formation of a single new product $\left(\mathbf{2 7} \mathbf{M n}_{2}{ }^{\mathbf{a z}}\right)$. Product $\mathbf{2 7} \mathbf{M n}_{\mathbf{2}^{\mathbf{a z}}}$ was isolated in $50 \%$ yield by filtration and subsequent crystallization from $\mathrm{CH}_{2} \mathrm{Cl}_{2}$ layered with methanol. The composition, as determined by HR-MS (MALDI), suggested that $\mathbf{2 7} \mathbf{M n}_{\mathbf{2}} \mathbf{a z}^{\mathbf{a z}}$ is a dimanganese complex of $\mathbf{2 7} \mathbf{H}_{4}\left(\mathrm{C}_{92} \mathrm{H}_{80} \mathrm{~N}_{11} \mathrm{Mn}_{2}{ }^{+}\right.$for $M^{+}$; Figure 28).

To facilitate the interpretation of spectra presented in the following chapters, syntheses of monomanganese complexes were conducted. Alas, no monomanganese complexes could be detected or isolated. This is attributed to a positive allosteric effect upon coordination of the first manganese ion. The favored six-fold coordination of the manganese ion evokes a relaxation of the macrocyclic twist. This unwinding of the macrocycle increases the accessibility of the second coordination pocket and facilitates so the formation of bimetallic complexes over monometallic ones.

Alternatively, an indirect access to complexes bearing only one manganese ion via the prior preparation of mononickel complex $27 \mathbf{H}_{2} \mathbf{N i}$ was chosen. Synthesis of complex $\mathbf{2 7} \mathbf{H}_{2} \mathbf{N i}$ of the Siamese-twin porphyrin was established by BLUSCH and employed further to access similar heterobimetallic species such as $\mathbf{2 7} \mathbf{N i C u}$. ${ }^{[82]}$

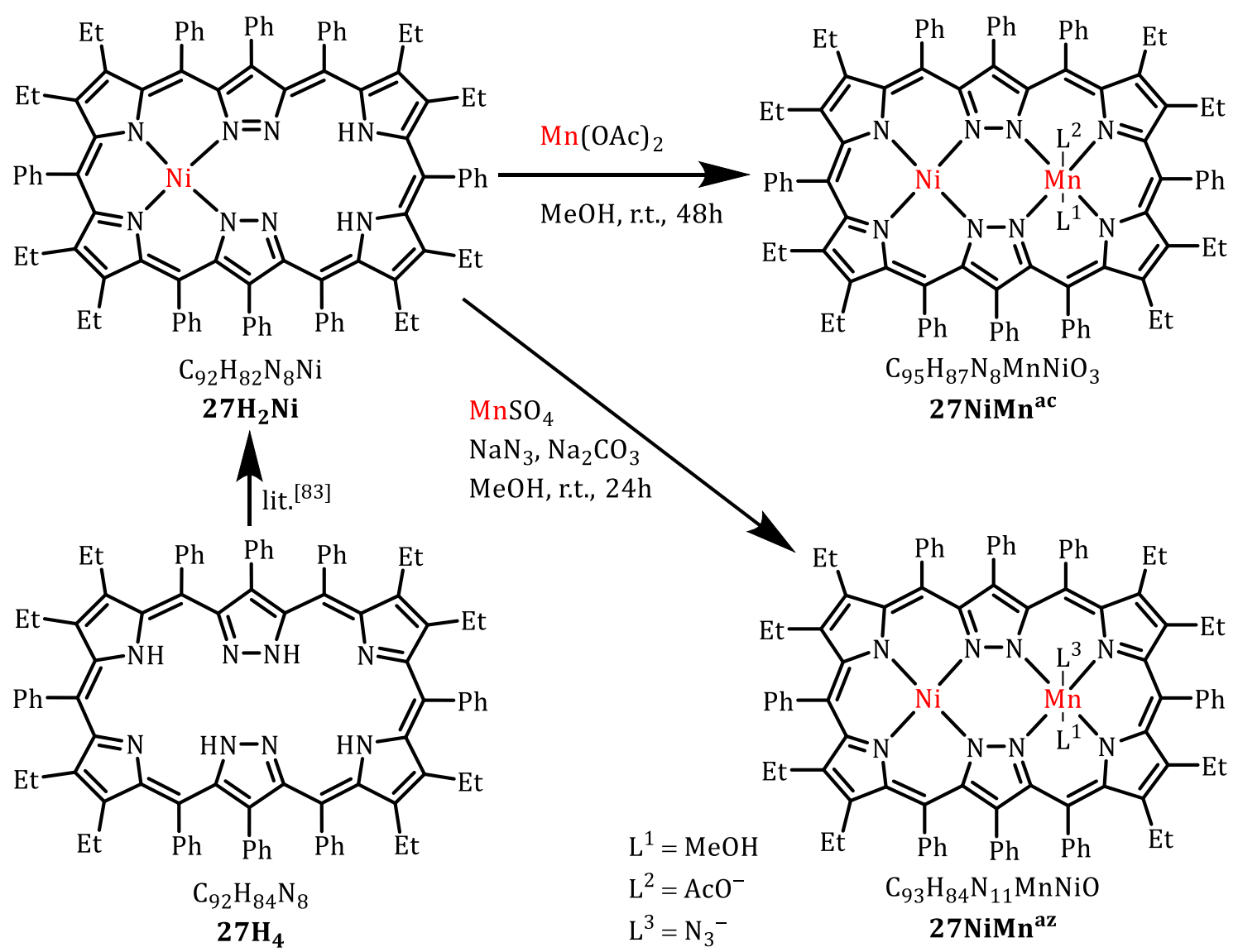

Scheme 15: Synthesis of heterobimetallic complexes 27NiMnac and 27NiMnaz. 
Reaction of blue-green complex $\mathbf{2 7} \mathbf{H}_{2} \mathbf{N i}$ (Scheme 15) in a mixture of $\mathrm{CH}_{2} \mathrm{Cl}_{2} / \mathrm{MeOH}$ with $\mathrm{Mn}(\mathrm{OAc})_{2}$ at room temperature overnight led to a brown-colored reaction solution. TLC indicated the consumption of the mononickel complex $2 \mathbf{2 H}_{2} \mathbf{N i}$ and mass spectrometry indicated the formation of a single new product (27NiMnac). Product 27NiMnac was isolated in $70 \%$ yield by evaporation and subsequent filtration through a short column of neutral alumina with EtOAc as eluent. Complex 27 NiMnaz was synthesized analogously using $\mathrm{MnSO}_{4}$, $\mathrm{NaN}_{3}$, and $\mathrm{Na}_{2} \mathrm{CO}_{3}$ (yield: $84 \%$ ). The composition, as determined by HR-MS (ESI ${ }^{+}$), suggested that $\mathbf{2 7} \mathrm{NiMn}^{\text {ac }}$ and $\mathbf{2 7} \mathbf{N i M n}{ }^{\mathrm{az}}$ are nickel manganese complexes of $\mathbf{2 7} \mathbf{H}_{4}$ (both $\mathrm{C}_{92} \mathrm{H}_{80} \mathrm{~N}_{8} \mathrm{NiMn}^{+}$ for $M^{+}$; Figure 29).
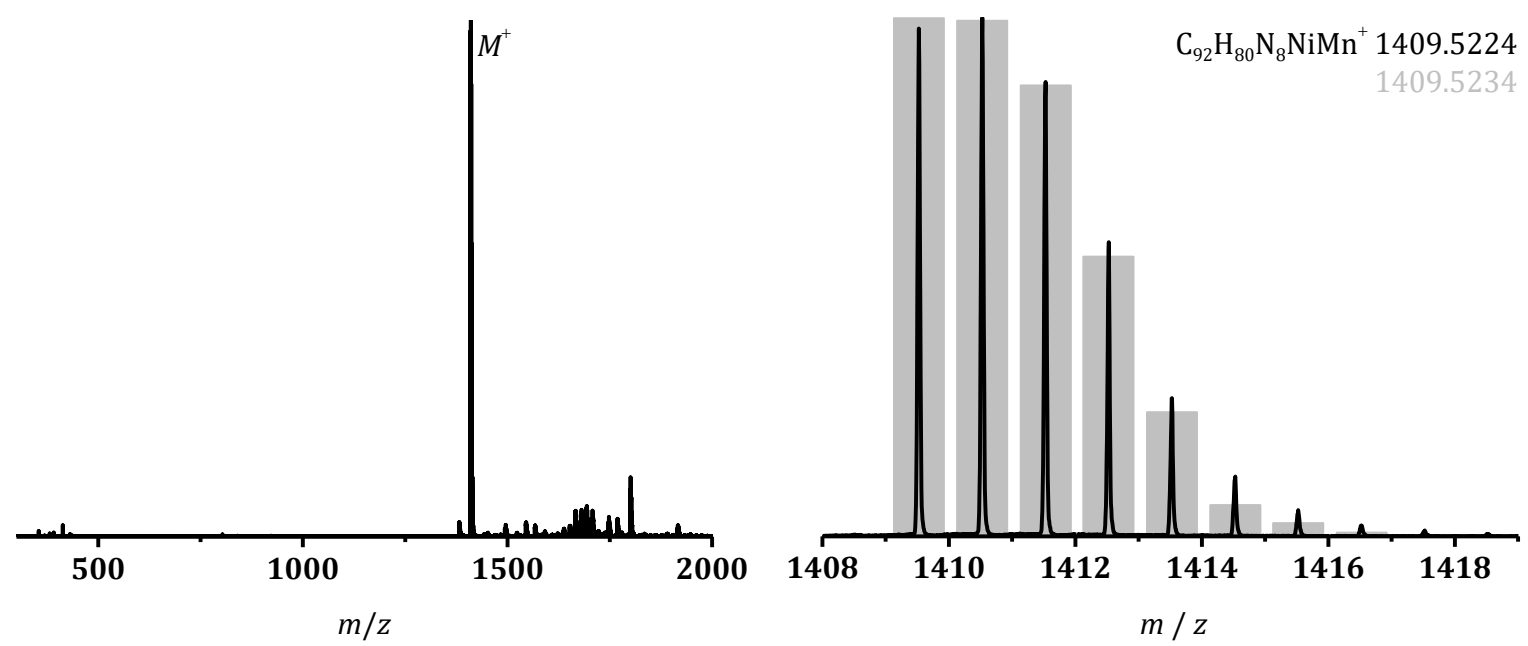

Figure 29: $\mathrm{HR}^{-\mathrm{ESI}^{+}}$spectrum of $27 \mathrm{NiMnac}$ and the isotopic distribution of the molecular peak $\mathrm{M}^{+}$(simulation in grey).

\subsubsection{UV/vis-NIR Spectral Characterization}

UV/vis-NIR spectra of the complexation products $\mathbf{2 7} \mathbf{M n}_{\mathbf{2}^{\text {ac }}}$ and $\mathbf{2 7} \mathbf{\mathbf { M n }} \mathbf{2}^{\mathrm{az}}$ (Figure 30, Table 5) are similar in shape. As observed for the parent molecule $\mathbf{2 7} \mathbf{H}_{\mathbf{4}}$, both complexes show strong

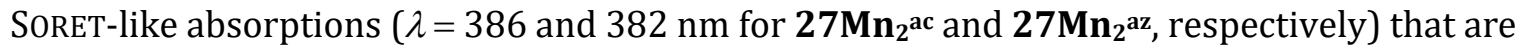
slightly blue-shifted with regard to the free-base ligand $\mathbf{2 7} \mathbf{H}_{\mathbf{4}}$. Conversely, the strong absorption at longer wavelengths shows a large bathochromic shift $(\Delta \lambda=47$ and $54 \mathrm{~nm}$ for
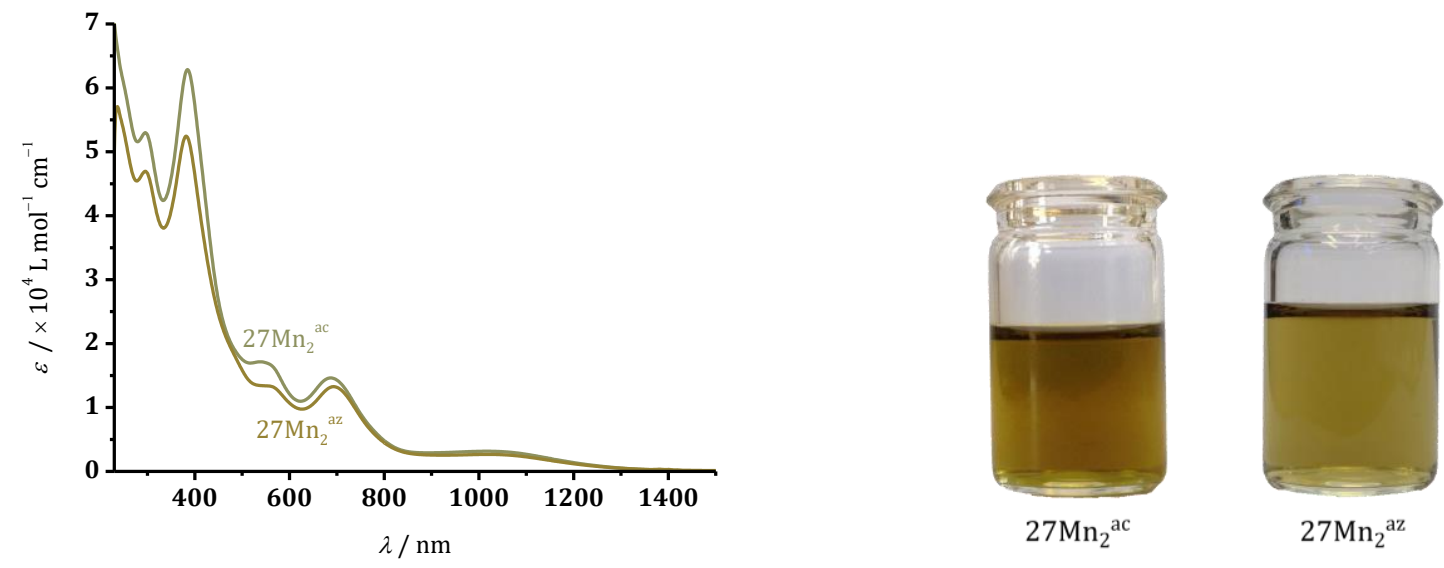

Figure 30: UV/vis-NIR spectra $\left(298 \mathrm{~K}, \mathrm{CH}_{2} \mathrm{Cl}_{2}\right)$ of manganese complexes $\mathbf{2 7} \mathbf{M n}_{2}{ }^{\text {ac }}$ (green trace) and $\mathbf{2 7} \mathbf{M n} \mathbf{n}^{\text {az }}$ (olive-green trace). 
$\mathbf{2 7} \mathbf{M n}_{\mathbf{2}^{\mathrm{ac}}}$ and $\mathbf{2 7} \mathbf{M n}_{\mathbf{2}^{\mathrm{az}}}$, respectively). This observation is consistent with the established understanding[83] that, on the one hand, contributions to the SORET-like absorption in Siamese-twin porphyrin complexes are mainly ligand-based $\left(\pi \rightarrow \pi^{*}\right)$ and thus less affected by metal coordination. On the other hand, charge-transfer (CT) transitions contribute to bands above $\lambda \approx 400 \mathrm{~nm}$ and are highly dependent on the nature of the coordinated metal ion. Additionally, the broadness of the low-energy bands suggests an overlapping of multiple transitions.

Table 5: UV/vis-NIR spectral data* of free-base Siamese-twin porphyrin $2 \mathbf{2 H}_{\mathbf{4}}$ and its homo- and heterobimetallic

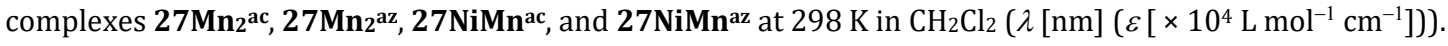

\begin{tabular}{ccccc}
\hline $\mathbf{2 7} \mathbf{H}_{\mathbf{4}}$ & $\mathbf{2 7 M n}_{\mathbf{2}^{\mathbf{a c}}}$ & $\mathbf{2 7 M n}_{\mathbf{a}^{\mathbf{a z}}}$ & $\mathbf{2 7 N i M n}^{\mathbf{a c}}$ & $\mathbf{2 7 N i M n}^{\mathbf{a z}}$ \\
\hline $305(2.9)$ & $295(5.3)$ & $295(4.7)$ & $277(2.9)$ & $288(4.6)$ \\
$389(7.7)$ & $386(6.3)$ & $382(5.2)$ & $382(4.0)$ & $376(6.5)$ \\
- & $544(1.7)$ & $552(1.3)$ & $530(1.3)$ & $504(2.2)$ \\
$641(3.4)$ & $688(1.4)$ & $695(1.3)$ & $709(1.1)$ & $704(1.8)$ \\
$740(1.3)$ & - & - & - & - \\
- & $1013(0.3)^{\mathrm{a}}$ & $1018(0.3)^{\mathrm{b}}$ & $1200(0.1)^{\mathrm{c}}$ & $1211(0.2)^{\mathrm{c}}$ \\
\hline
\end{tabular}

* full width at half maximum (FWHM): a $\Delta v_{1 / 2}=29700 \mathrm{~cm}^{-1}$; b $\Delta v_{1 / 2}=31100 \mathrm{~cm}^{-1}$; $\Delta v^{1 / 2}=23400 \mathrm{~cm}^{-1}$;

The exact location of the absorption bands further reflects not only sensitivity toward the

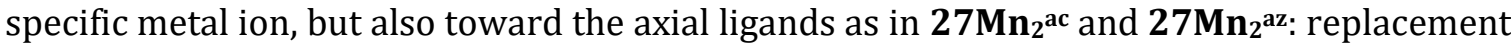
of the acetate by an azide leads to a significant bathochromic shift in all major features of the UV/vis-NIR spectrum. This effect can be attributed to the difference in ligand strength according to the spectrochemical series $\left(\mathrm{N}_{3}{ }^{-}<\mathrm{AcO}^{-}\right)$and the type of ligand bond ( $\sigma-\mathrm{vs} \pi-$ bond).[107]

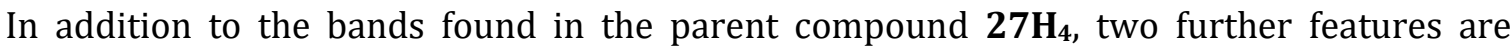
observed in the UV/vis-NIR spectra of $\mathbf{2 7 M \mathbf { n } _ { 2 }}$ ac and $\mathbf{2 7} \mathbf{M n}_{\mathbf{2}^{\mathrm{az}}}$ : a new band at $\lambda \approx 550 \mathrm{~nm}$ and a broad absorption in the NIR region of the electromagnetic spectrum with $\lambda=1013$ and

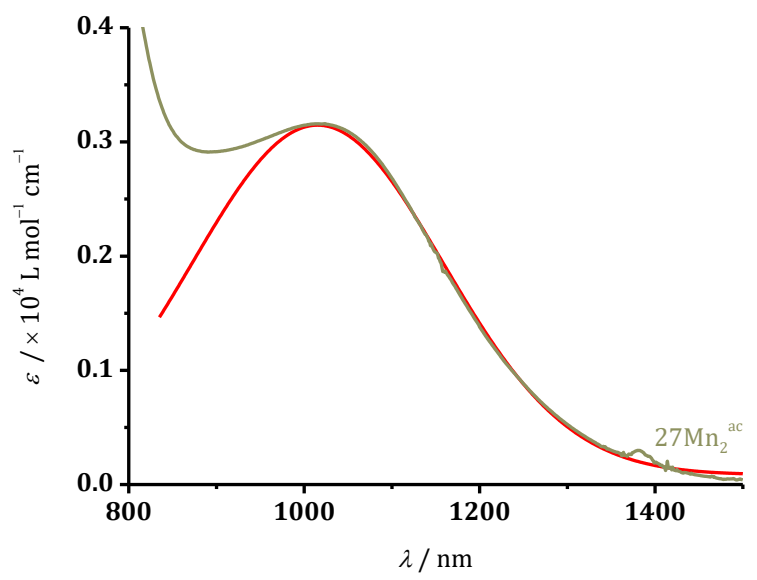

\begin{tabular}{lrrrr}
\multicolumn{5}{c}{$y=y_{0}+\frac{A}{w \sqrt{\pi / 2}} e^{-2\left(x-x_{c} / w\right)^{2}}$} \\
\hline \\
\hline y0 & $27 \mathrm{Mn} 2 \mathrm{ac}$ & $\frac{27 \mathrm{Mn} 2 \mathrm{az}}{27 \mathrm{NiMnac}}$ & $\frac{27 \mathrm{NiMnaz}}{}$ \\
$\mathrm{xc}$ & 85.20 & 178.89 & 777.41 & 115.78 \\
$\mathrm{w}$ & 1015.33 & 1020.38 & 1200.23 & 1211.26 \\
$\mathrm{~A}$ & 285.68 & 273.67 & 363.09 & 363.73 \\
sigma & 109623.00 & 840892.41 & 563575.05 & $1.04 \mathrm{E}+06$ \\
FWHM & 142.84 & 136.84 & 181.54 & 181.87 \\
Height & 336.36 & 322.23 & 427.50 & 428.26 \\
\hline
\end{tabular}

Figure 31: Representative curve fitting for NIR absorption band for complex 27Mnn $\mathbf{2}^{\text {ac }}$ (green trace). Curve fit (red trace) and parameters were calculated using the indicated Gaussian fitting function. Curve fits for $\mathbf{2 7} \mathbf{M n}_{2} \mathbf{a z}^{\mathbf{a z}}$, 27NiMnac and 27NiMnaz are summarized in Figure S9. 
$1018 \mathrm{~nm}$ for $\mathbf{2 7} \mathbf{M n}_{\mathbf{2}^{\mathrm{ac}}}$ and $\mathbf{2 7} \mathbf{M n}_{\mathbf{2}^{\mathrm{az}}}$, respectively. Gaussian curve fitting was applied to the low-energy bands according to Figure 31 to reliably extract parameters such as the wavelength $\left(\lambda_{\max }\right)$, extinction coefficient $(\varepsilon)$ and full width at half maximum $\left(\Delta v_{1 / 2}\right)$.

Such broad absorptions (Table 5) are commonly associated with ligand-bridged mixed-valent complexes (e.g. $\mathrm{M}^{1}$ (II)-L-M ${ }^{2}$ (III)) that undergo an intervalence charge transfer (IVCT) ${ }^{[108]}$ due to dynamic delocalization of charge between both metal centers. However, the Mn(III)-Mn(III) couple cannot be suitable for the process at hand. ${ }^{*}$ To remedy this issue, the band at $\lambda \approx 550 \mathrm{~nm}$ has to be taken into account. As has been shown by BLUSCH for $\mathbf{2 7} \mathbf{N i C u}$ and $\mathbf{2 7} \mathbf{N i}_{2},{ }^{[82]}$ absorptions in this region become particularly pronounced with progressing ligand oxidation during spectroelectrochemical investigations and EPR spectroscopy revealed significant spin delocalization between the central ion and the ligand.

Consequently, it is reasonable to assume an intermittent Mn(II)- $\mathrm{L}^{++}$electronic state for each binding pocket in complexes $\mathbf{2 7} \mathbf{M n}_{2}{ }^{\text {ac }}$ and $\mathbf{2 7} \mathbf{M n}_{2}{ }^{\text {az }}$ as a result of an intramolecular electron transfer from the ligand to the metal. The formation of such porphyrin $\pi$-radical cations has been observed and analyzed in detail for the di-heme enzyme MauG and its model complexes bearing the isoelectronic subunit Fe(III)- $\mathrm{L}^{\cdot+}{ }^{+}[109-111]$ Broad NIR absorption bands $\left(h v_{\mathrm{CR}}\right)$ are considered a characteristic spectral feature for the presence of interacting $\pi$-radical cations and are attributed to charge resonance (CR) phenomena (Scheme 16).[111-114] The CR stabilization energy $\left(\Delta E_{\mathrm{CR}}\right)$ originates from exchange interactions between molecular orbitals of each porphyrinic subunit and subsequent delocalization of the spin and charge over a greater number of atoms.[109]

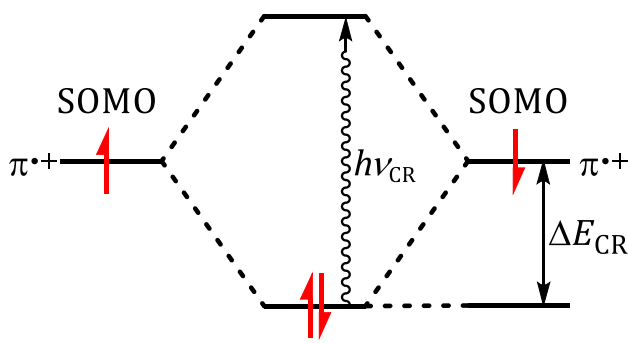

type I

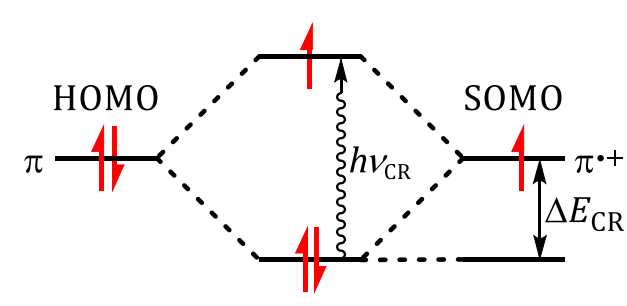

type II

Scheme 16: Representation for the origin of charge resonance stabilization energy $\left(\Delta E_{\mathrm{CR}}\right)$ and therewith associated CR bands $\left(\boldsymbol{h} \boldsymbol{v}_{\mathrm{CR}}\right)$ in the NIR: On the left, interaction of two $\pi$-radical cations (type I CR) as proposed for

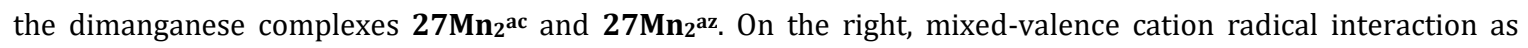
proposed for nickel manganese complexes 27NiMnac and 27NiMnaz.[111,112,114]

Thus, in case of the dimanganese complexes $\mathbf{2 7} \mathbf{M n}_{\mathbf{2}^{\mathrm{ac}}}$ and $\mathbf{2 7} \mathbf{M} \mathbf{n}_{\mathbf{2}^{\mathrm{az}}}$ a type I interaction (Scheme 16) may be expected. The $\pi$-radicals centered on the conjugated systems of both coordination pockets are stabilized by an interaction of their respective singly occupied molecular orbitals (SOMOs).[109] It seems, the same pyrazole moiety that interrupts the overall macrocyclic conjugation in the free-base Siamese-twin porphyrin $\mathbf{2 7} \mathbf{H}_{\mathbf{4}}$ enables an electronic

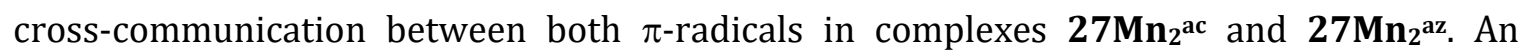

\footnotetext{
${ }^{*}$ Both manganese ions are assigned the formal oxidation state + III according to X-ray crystallographic analyses as will be detailed in Chapter 3.3.3.
} 


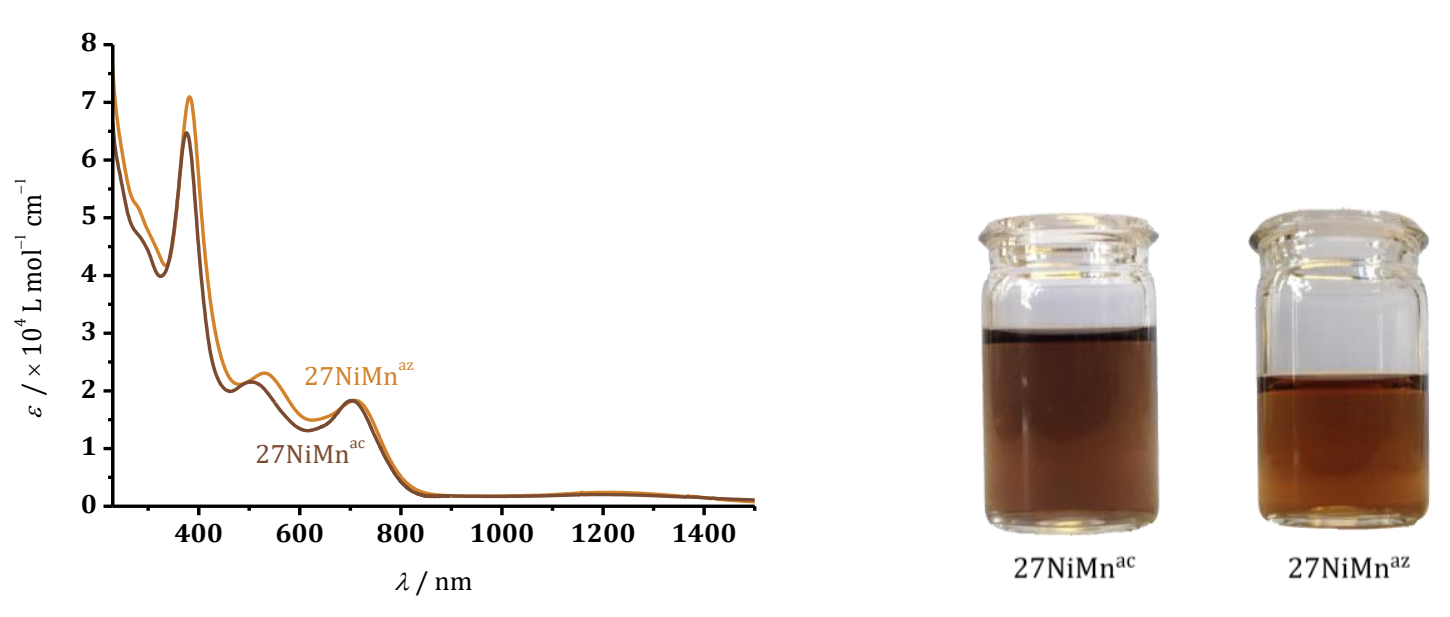

Figure 32: UV/vis-NIR spectra $\left(298 \mathrm{~K}, \mathrm{CH}_{2} \mathrm{Cl}_{2}\right)$ of nickel manganese complexes 27NiMnac (brown trace) and 27NiMnaz (orange-brown trace).

analogous stabilization (Scheme 16, type II) may occur in nickel manganese complexes 27 NiMnac and 27NiMnaz through an interaction of a $\pi$-radical centered on the manganese coordinating half of the Siamese-twin porphyrin with a closed-shell highest occupied molecular orbital (HOMO) centered on the nickel coordinating half of the ligand.[109] Despite the inherent energy penalty caused by electrostatic repulsion in type I complexes, the CR stabilization energy of $\pi$-diradicals is larger than that of mixed-valence type II complexes, which results in a red-shift of the CR band for the latter.[109,111] And indeed, UV/vis-NIR spectral analyses of the heterobimetallic complexes 27 NiMnac and 27 Ni Mnaz (Figure 32, Table 5) show a CR band in the NIR region of the electromagnetic spectrum with a large shift

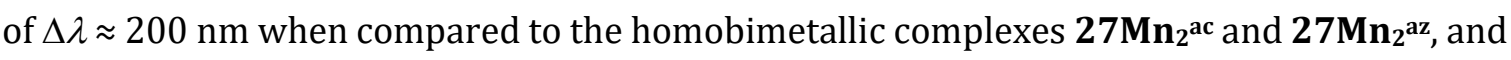
little other fundamental changes in the visible range.

Despite the similarities to literature examples so far, the origin of the NIR bands remains speculative and further investigations into the nature of these bands (e.g. concentration, solvent and temperature dependence studies; complementary DFT calculations) are needed. Nonetheless, occurrence of a CR band for both homobimetallic complexes $\mathbf{2 7} \mathbf{M n}_{\mathbf{2}}$ ac and $\mathbf{2 7} \mathbf{M n}_{2}{ }^{\text {az }}$ and heterobimetallic complexes $\mathbf{2 7} \mathbf{N i M n}{ }^{\text {ac }}$ and $\mathbf{2 7} \mathbf{N i M n}{ }^{\text {az }}$ is the first indication of an electronic interaction of the redox-active metal ion with a redox non-innocent Siamese-twin

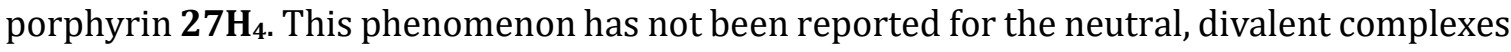
of copper and nickel ions.[84]

\subsubsection{X-ray Crystallographic Molecular Structures}

Single crystals of the dimanganese complex $\mathbf{2 7} \mathbf{M n}_{\mathbf{2}^{\text {ac }}}$ suitable for X-ray diffraction were obtained by slow evaporation of a saturated solution of $\mathbf{2 7} \mathbf{M n}_{2} \mathbf{2}^{\text {ac }}$ in $\mathrm{MeOH}$. The diffraction data were resolved in the non-chiral, monoclinic space group $C 2 / c$ (Figure 33; Chapter 6.1). The structure shows the Siamese-twin porphyrin scaffold with two octahedrally coordinated manganese ions. Each metal ion bears an acetate and a methanol molecules in its axial positions with trans-orientation regarding the mean plane of the macrocycle.

Taking into account the dibasicity of each coordination pocket, the charge of the axial ligands, and the lack of any further counter ions, the manganese ion is assigned the formal oxidation state + III. In situ oxidation of the central ion is consistent with reported metalation reactions 
of porphyrins that, despite the use of a manganese(II) salts, inevitably yield manganese(III) porphyrin complexes.[115]

The ligand scaffold is slightly more relaxed in comparison to the free-base parent molecule ( $\tau^{\mathrm{av}}= \pm 95^{\circ}$ for $\mathbf{2 7} \mathbf{M n}_{2}$ ac vs $\tau= \pm 97^{\circ}$ for $\mathbf{2 7 \mathbf { H } _ { 4 }}$ ) conceding space for the coordination of axial

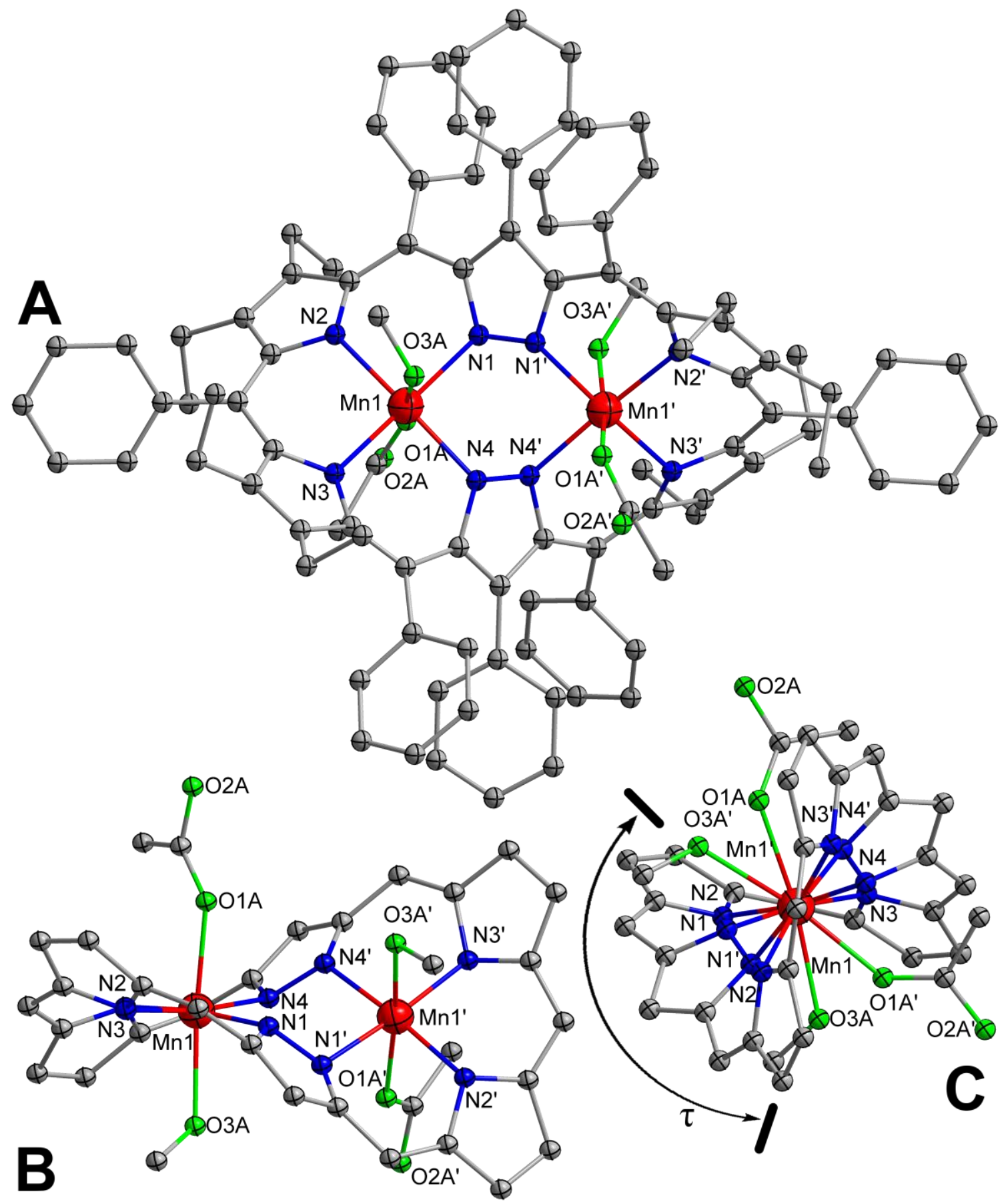

Figure 33: Ball-and-stick representation of the solid state structure of the dimanganese acetato complex of the Siamese-twin porphyrin $\mathbf{2 7} \mathbf{M n}_{2} \mathbf{2}^{\text {ac }}$ (manganese in red, oxygen in green, carbon in grey, and nitrogen in blue). Only one enantiomer shown; solvent molecules and hydrogen atoms omitted for clarity. A) Overview including peripheral substituents; B) representation of the $C_{2}$-symmetry of the structure highlighting the trans-location of axial ligands, and $\mathrm{C}$ ) view along the long axis depicting the torsion angle $\tau$. Symmetry transformation for equivalent atoms ('): $1-x, y, 1.5-z$. 
ligands (Table 6). In general, the alternating short-long pattern in bond lengths of the two individual conjugation pathways is preserved upon metalation (cf. Chapter 6.1). Only slight averaging between the single and double bonds of the dipyrrin unit is observed. This particular section of the macrocycle was already shown to play a key role in the redox

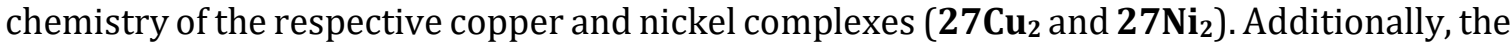
averaging supports further the proposed charge delocalization between the central ion and the ligand scaffold (cf. CR band in Figure 30). The helical pitch is shortened marginally, adjusting to the electrostatic pull of the central ion on the coordinating subunits.

Table 6: Structural parameters for $27 \mathrm{H}_{4}, 27 \mathrm{Mn}_{2}{ }^{\mathrm{ac}}$, and $27 \mathrm{Mn}_{2}{ }^{\mathrm{az}}$.

\begin{tabular}{|c|c|c|c|}
\hline & $27 \mathrm{H}_{4}$ & $27 \mathrm{Mn}_{2}{ }^{\mathrm{ac}}$ & $27 \mathrm{Mn}_{2}{ }^{\mathrm{az}}$ \\
\hline$\tau\left[\left[^{\circ}\right]\right.$ & \pm 97 & $\pm 94 / \pm 96$ & \pm 93 \\
\hline$d_{\text {helix }}[\AA ̊]$ & 10.78 & 10.66 & 10.74 \\
\hline$d_{\text {trans }}(\mathrm{N} \cdots \mathrm{N})[\AA]$ & $-\mathrm{a}$ & $4.08 / 4.06$ & $4.07 / 4.04$ \\
\hline$d_{\min }(\mathrm{C}-\mathrm{C})[\AA]$ & 1.36 & 1.36 & 1.37 \\
\hline$d_{\max }(\mathrm{C}-\mathrm{C})[\AA]$ & 1.48 & 1.47 & 1.46 \\
\hline$d(\mathrm{Mn} \cdots \mathrm{Mn})[\AA]$ & - & 3.96 & 4.01 \\
\hline$d(\mathrm{Mn}-\mathrm{N})[\AA]$ & - & $2.02 / 2.03 / 2.04 / 2.05$ & $2.00 / 2.03 / 2.04 / 2.05$ \\
\hline$\Varangle(\mathrm{NMnN})\left[^{\circ}\right]$ & - & 89 / 92 / 89 / 91 & $90 / 91 / 91 / 89$ \\
\hline$d\left(\mathrm{Mn}-\mathrm{L}^{\mathrm{ax}}\right)[\AA]$ & - & $2.30(\mathrm{MeOH}) / 2.23\left(\mathrm{AcO}^{-}\right)$ & $2.30(\mathrm{MeOH}) / 2.31$ and $2.09\left(\mathrm{~N}_{3}{ }^{-}\right)$ \\
\hline
\end{tabular}

a The measurement was omitted due to the lack of a square-planar arrangement of the nitrogen atoms caused by the severe twist of the macrocyclic backbone (compare Figure 13).

The manganese(III) ion is located in the center of a stretched octahedron with an in-plane position regarding the $\left\{\mathrm{N}_{4}\right\}$ mean plane. The axial JAHN-TELLER distortion agrees well with a high-spin $\mathrm{d}^{4}$ electron configuration: the partially occupied $e_{\mathrm{g}}$-orbital set undergoes a splitting of the initially degenerate orbitals upon elongation of bonds to the axial ligands $\left(O_{\mathrm{h}} \rightarrow D_{4 \mathrm{~h}}\right)$, thus lowering the energy of the system. Considering the partial charge delocalization as the basis for CR phenomena observed in solution (cf. Chapter 3.3.2), a dynamic JAHN-TELLER distortion must be assumed.
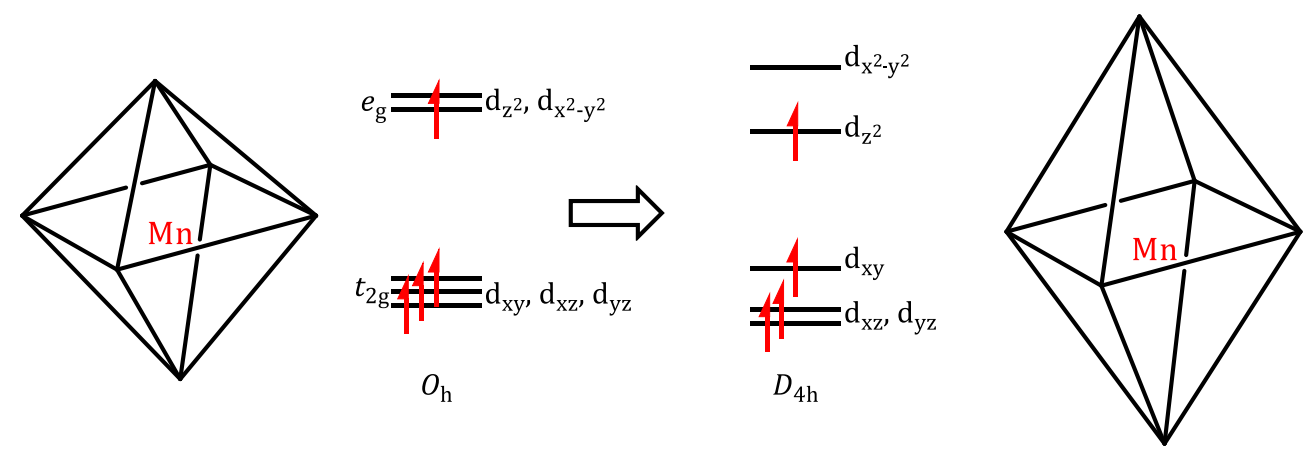

Scheme 17: Orbital splitting in an idealized octahedral $\left(O_{\mathrm{h}}\right.$, left $)$ and JAHN-TELLER elongated ( $D_{4 \mathrm{~h}}$, right) coordination geometry for a $\mathrm{d}^{4}$ electron configuration. ${ }^{[1]}$ 
Single crystals of the dimanganese complex $\mathbf{2 7} \mathbf{M n}_{\mathbf{2}^{\mathrm{az}}}$ suitable for X-ray diffraction were obtained by layering of a saturated solution of $\mathbf{2 7} \mathbf{M n}_{2}{ }^{\text {az }}$ in $\mathrm{CH}_{2} \mathrm{Cl}_{2}$ with $\mathrm{MeOH}$. The diffraction data were resolved in the non-chiral, monoclinic space group C2/c (Figure 34; Chapter 6.1). The structure shows, as already observed for $\mathbf{2 7} \mathbf{M n}_{2}$ ac, the twisted Siamese-twin porphyrin

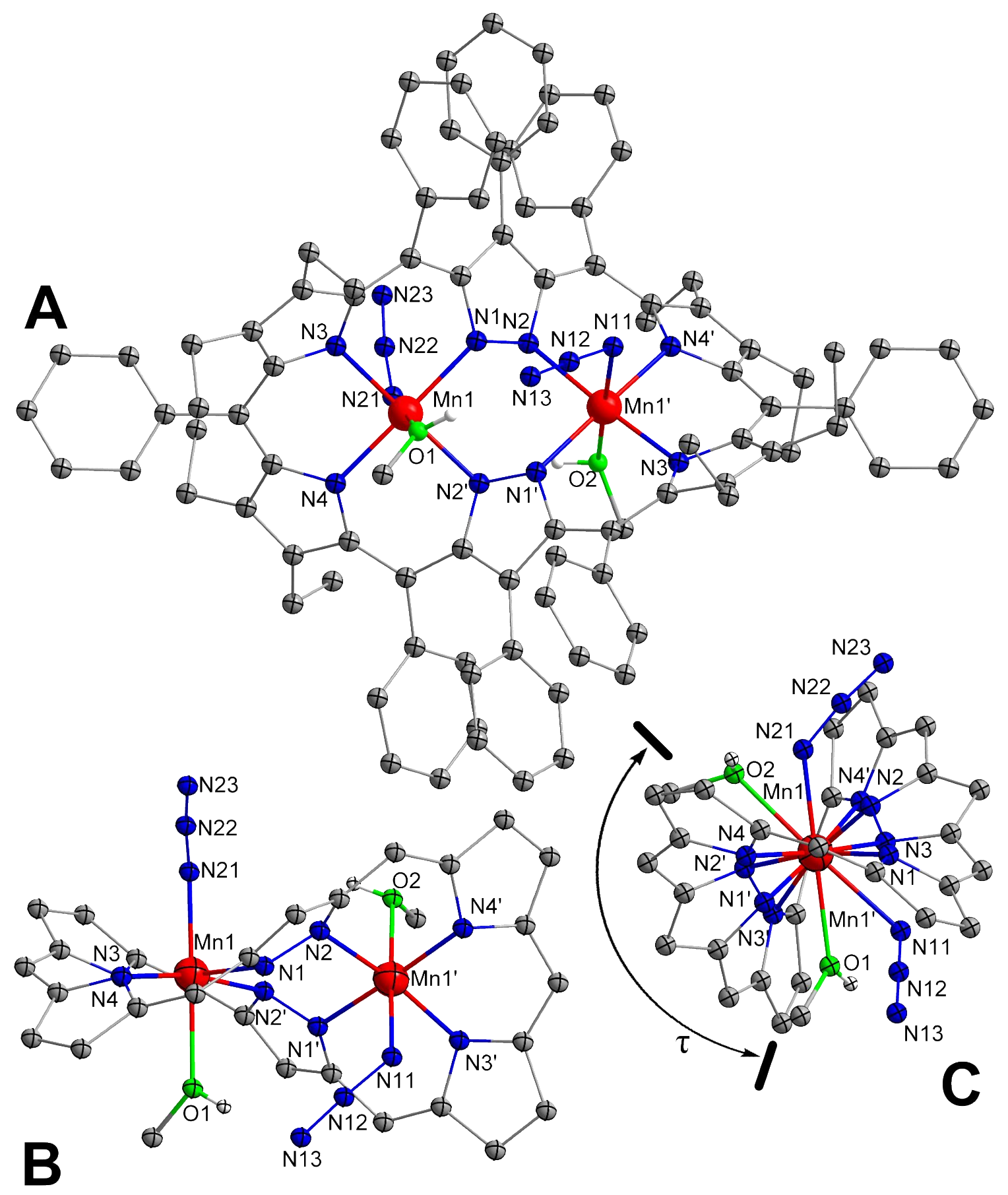

Figure 34: Ball-and-stick representation of the solid state structure of the dimanganese azido complex of the Siamese-twin porphyrin $\mathbf{2 7} \mathbf{M n}_{\mathbf{2}^{\mathrm{az}}}$ (manganese in red, oxygen in green, carbon in grey, nitrogen in blue and hydrogen in white). Only one enantiomer shown; solvent molecules and carbon-bound hydrogen atoms omitted for clarity. A) Overview including peripheral substituents; B) representation of the $C_{2}$-symmetry of the core structure highlighting the random orientation of axial ligands, and $C$ ) view along the long axis depicting the torsion angle $\tau$. Symmetry transformation for equivalent core atoms ('): $1-x, y, 1.5-z$. 
scaffold coordinating two manganese(III) ions with trans-oriented azide and methanol molecules as axial ligands. Aside from the position of the anionic axial ligand $\left(\mathrm{AcO}^{-}\right.$and $\mathrm{N}_{3}{ }^{-}$), all structural parameters (Table 6) vary insignificantly from each other. In contrast to the acetate ion, with an unvarying distance to the manganese ion of $d(\mathrm{Mn}-\mathrm{OAc})=2.23 \AA$ for both coordination pockets, the distance of the azide ion differs significantly within the same complex $\left(d\left(\mathrm{Mn}-\mathrm{N}_{3}\right)=2.31\right.$ and $2.09 \AA$; cf. Chapter 6.1). In addition, both azide ions are orientated randomly with regard to the macrocyclic scaffold (Figure 34B). This observation underlines once again the lower ligand strength of the azide ligand in comparison to the acetate according to the spectrochemical series.[107]

In addition, single crystals of the dimanganese complex $\mathbf{2 7} \mathbf{M n}_{\mathbf{2}^{\mathrm{az}}}$ suitable for X-ray diffraction were obtained by layering of a saturated solution of $\mathbf{2 7} \mathbf{M n}_{2}$ az in THF with $\mathrm{MeOH}$ (Chapter 6.1). Apart from the axial coordination, where a THF molecule is coordinated instead of methanol, all structural parameters show only marginal deviations.

The coordination environment of the manganese(III) ion in both complexes $\mathbf{2 7} \mathbf{M n}_{2}$ ac and $\mathbf{2 7} \mathbf{M n}_{2}{ }^{\text {az }}$ is in good agreement with reported manganese(III) porphyrin complexes.[115-117] Especially the comparison of bond lengths between the manganese ion and the equatorial nitrogen donor atoms (Table 6) supports the correct assignment of the oxidation state. Manganese(II) porphyrin complexes show longer bond lengths of $d(\mathrm{Mn}(\mathrm{II})-\mathrm{N})=2.12-2.13 \AA$ as a result of the high-spin $d^{5}$ electron configuration and therefore occupation of the $d_{x^{2}-y^{2}}$ orbital (cf. Scheme 17).[118] In contrast, corresponding bond lengths in complexes $\mathbf{2 7} \mathbf{M n}_{\mathbf{2}} \mathbf{a c}^{\text {ac }}$ and $\mathbf{2 7} \mathbf{M n}_{\mathbf{2}^{\mathrm{az}}}$ fall within the reported range of $d(\mathrm{Mn}(\mathrm{III})-\mathrm{N})=2.00-2.03 \AA .[116,117]$

Single crystals of the nickel manganese complex $\mathbf{2 7} \mathbf{N i M n}^{\text {ac }}$ suitable for X-ray diffraction were obtained by slow evaporation of a saturated solution of 27 NiMnac in MeOH. The diffraction data were resolved in the non-chiral, monoclinic space group $C$. However, strong disorder within the cell impeded a complete resolution of the structure particularly with respect to the occupation of the coordination pockets and the assignment of axial ligands. Due to the everpresent disorder in heterobimetallic complexes of the Siamese-twin porphyrin $\mathbf{2 7} \mathbf{M}^{1} \mathbf{M}^{2}$ no $\mathrm{X}$-ray crystallographic molecular structure of these complexes could be reported to date.

\subsubsection{Magnetic Susceptibility Measurements}

Magnetic properties of $\mathbf{2 7} \mathbf{M} \mathbf{n}_{2}{ }^{\text {ac }}$ and $\mathbf{2 7} \mathbf{M n}_{2}{ }^{\text {az }}$ were studied by susceptibility measurements using a superconducting quantum interference device (SQUID). The data were collected on powder samples applying a magnetic field of $B=0.5 \mathrm{~T}$ in a temperature range from 2 to $295 \mathrm{~K}$. The program julX written by BILL was used for the simulation and analysis of experimental data (Figure 35, Table 7).[119] Temperature-independent paramagnetism (TIP) and CuRIE-behaved paramagnetic impurities (PI) were included according to $\chi_{\text {calc }}=(1-\mathrm{PI}) \chi+\mathrm{PI} \chi_{\mathrm{i}}+\mathrm{TIP}$.

At room temperature $\left(T=295 \mathrm{~K}\right.$ ) an effective magnetic moment of $\mu_{\mathrm{eff}}=6.20$ and $6.56 \mu_{\mathrm{B}}$ (for $\mathbf{2 7} \mathbf{M n}_{2}{ }^{\text {ac }}$ and $\mathbf{2 7} \mathbf{M n}_{2}{ }^{\text {az }}$, respectively) was measured. These numbers correspond well to the calculated spin-only value $\left(\mu_{\text {eff }}=6.94 \mu_{\mathrm{B}}\right)$ for two uncoupled high-spin $\mathrm{d}^{4}$-Mn(III) ions $\left(S_{1}=S_{2}=2, g_{\mathrm{e}}=2.0023\right)$ considering the lower LANDÉ factors $g=1.87$ and 1.93 (Table 7) determined by the calculated curve fit (Figure 35). Upon lowering the temperature, the effective magnetic moments decreased successively to $\mu_{\text {eff }}=0.98$ and $1.41 \mu_{\mathrm{B}}$ at $T=2 \mathrm{~K}$. The curve shape indicates an antiferromagnetic interaction (with a total spin of $S_{t}=0$ at low 
temperatures) between the manganese(III) ions. The experimental data were simulated by means of the isotropic HEISENBERG-DIRAC-VAN VLECK Hamiltonian supplemented by a ZEEMAN term (Equation (2)).[120]

$$
\widehat{H}=-2 J \vec{S}_{1} \vec{S}_{2}+\vec{S}_{1} g \mu_{B} \vec{B}\left(\vec{S}_{1}+\vec{S}_{2}\right)
$$

The calculated curve fit revealed small antiferromagnetic coupling constants $J=-3.9$ and

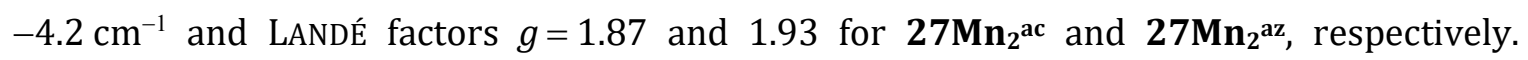
Antiferromagnetic interactions in the range of $J=-(2.7-4.2) \mathrm{cm}^{-1}$ were already observed before in pyrazole-bridged manganese(III) complexes.[121-123] Thus, the findings for the presented Siamese-twin porphyrin complexes are consistent with literature.

Table 7: Simulation parameters for $\mathbf{2 7} \mathbf{M n} 2^{\text {ac }}$ and $\mathbf{2 7} \mathbf{M n} 2^{\mathbf{a z}}(T=2-295 \mathrm{~K}$, solid sample) including the LANDÉ factor $g$, magnetic coupling constant $J$, and quantification of the paramagnetic impurity (PI) factored into the curve fit.

\begin{tabular}{ccc}
\hline & $\mathbf{2 7} \mathbf{M n}_{2}{ }^{\mathbf{a c}}$ & $\mathbf{2 7 M n}_{2} \mathbf{M z}^{\mathbf{a z}}$ \\
\hline$g$ & 1.87 & 1.93 \\
$J$ & $-3.9 \mathrm{~cm}^{-1}$ & $-4.2 \mathrm{~cm}^{-1}$ \\
PI & $3.6 \%$ & $5.4 \%$ \\
\hline
\end{tabular}

Nonetheless, the values differ from data reported for the dicopper complex of the Siamesetwin porphyrin $\mathbf{2 7} \mathrm{Cu}_{2}$, which exhibits a ferromagnetic coupling $\left.U=+16.3 \mathrm{~cm}^{-1}\right)$ between its copper(II) centers.[75] The reasoning of orthogonal magnetic orbitals $\left(\mathrm{d}_{\mathrm{x}^{2}-\mathrm{y}^{2}}\right.$ for $\mathrm{Cu}(\mathrm{II})$ ), appropriate in case of $\mathrm{d}^{9}$-Cu(II), has to be modified, since high-spin $\mathrm{d}^{4}-\mathrm{Mn}$ (III) not only has unpaired electrons in the $e_{\mathrm{g}}$ but also in the $t_{2 \mathrm{~g}}$ orbital set (Scheme 17). Thus, the antiferromagnetic coupling of the manganese(III) centers is attributed to the following effects: First, there is the $d \sigma$-interaction between the metal and the pyrazole-bridge, which is highly angle-dependent and becomes negligible for appropriate angles. Second, there is the $\mathrm{d} \pi$-interaction between the metal and the pyrazole-bridge, which is always different from zero and therefore contributes to the antiferromagnetic coupling. Consequently, the twisted

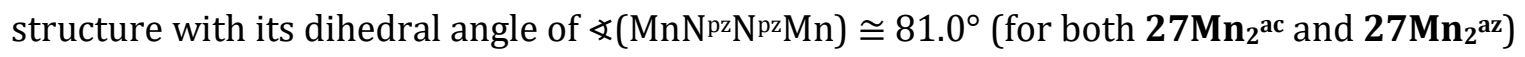
may attenuate the strength of the antiferromagnetic coupling but cannot change the sign of
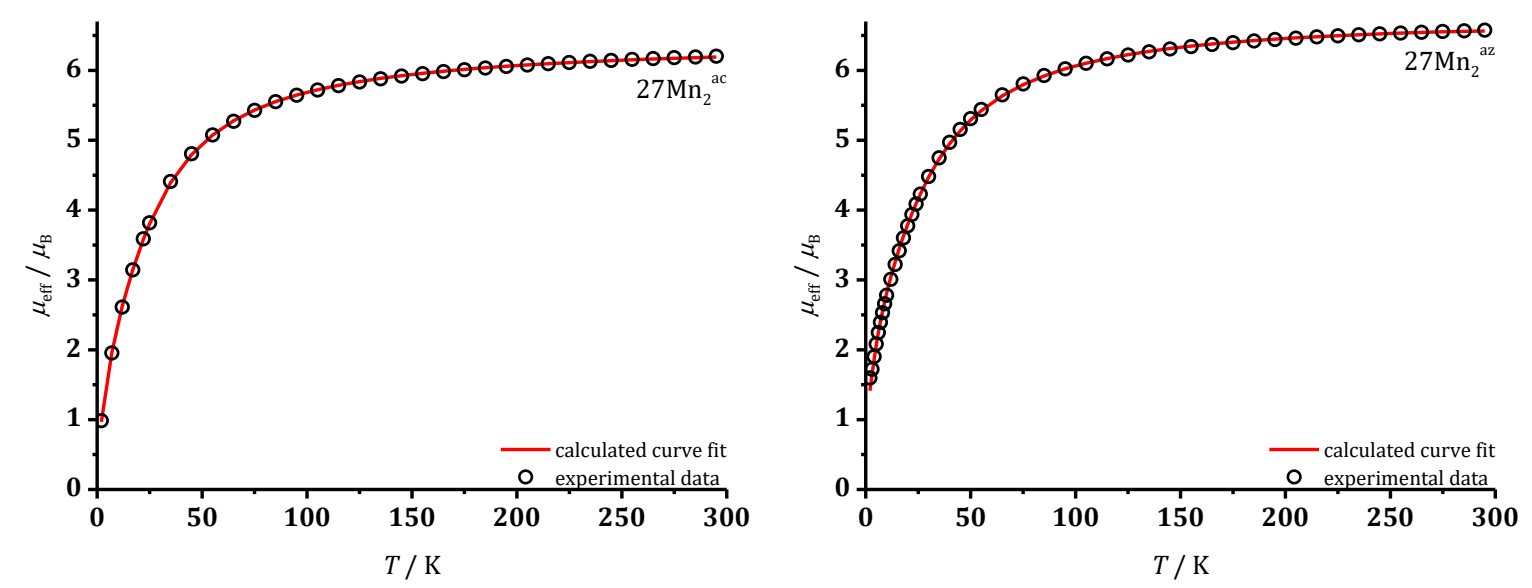

Figure 35: Plot of $\mu_{\text {eff }}$ vs $T$ (2-295 K, solid sample) for complexes $\mathbf{2 7} \mathbf{M n}_{\mathbf{2}^{\mathrm{ac}}}$ (left) and $\mathbf{2 7} \mathbf{M n}_{\mathbf{2}^{\mathbf{a z}}}$ (right), and the calculated curve fit $(-)$. 
the interaction.[1,124] In conclusion, all Mn(III) (and Mn(II)) complexes of $\mathbf{2 7} \mathbf{H}_{\mathbf{4}}$, in contrast to $\mathrm{Ni}(\mathrm{II})$ and $\mathrm{Cu}$ (II) complexes, should always be antiferromagnetic.[124]

The low LANDÉ factors are in line with data reported for manganese(III) porphyrins ( $g=1.82$ and 1.93) determined by high-field EPR spectroscopy by GOLDBERG as well as with data for some other manganese and pyrazole containing compounds. ${ }^{[123,125]}$ Since there is also a variety of reported $\mathrm{Mn}$ (III) complexes with $g \cong 2.00$ another minor contribution to the lowering of the LANDÉ factor in terms of diamagnetic impurities has to be considered.[121,122,126] Additional solvent molecules included in the crystal lattice and diamagnetic impurities may contribute to errors in the total molecular weight and therefore result in lower $g$-values when the fitting function is applied.

Magnetic susceptibility measurements were also conducted for the heterobimetallic complex 27NiMnac (Figure 36, left). The combination of a diamagnetic nickel(II) $\mathrm{d}^{8}$-ion and the paramagnetic manganese(III) $\mathrm{d}^{4}$-ion allows to eliminate any magnetic interaction as seen in case of $\mathbf{2 7} \mathbf{M n}_{2}$ ac and $\mathbf{2 7} \mathbf{M n}_{2}{ }^{\text {az }}\left(S_{t}=0\right.$ at low temperatures $)$ and solely probe the paramagnetic ion for its properties. While the susceptibility measurement confirmed a spin state of $S=2$ for 27NiMnac, a variable temperature variable field measurement (VTVH; Figure 35, right) was conducted to determine the size of the zero field splitting (ZFS).
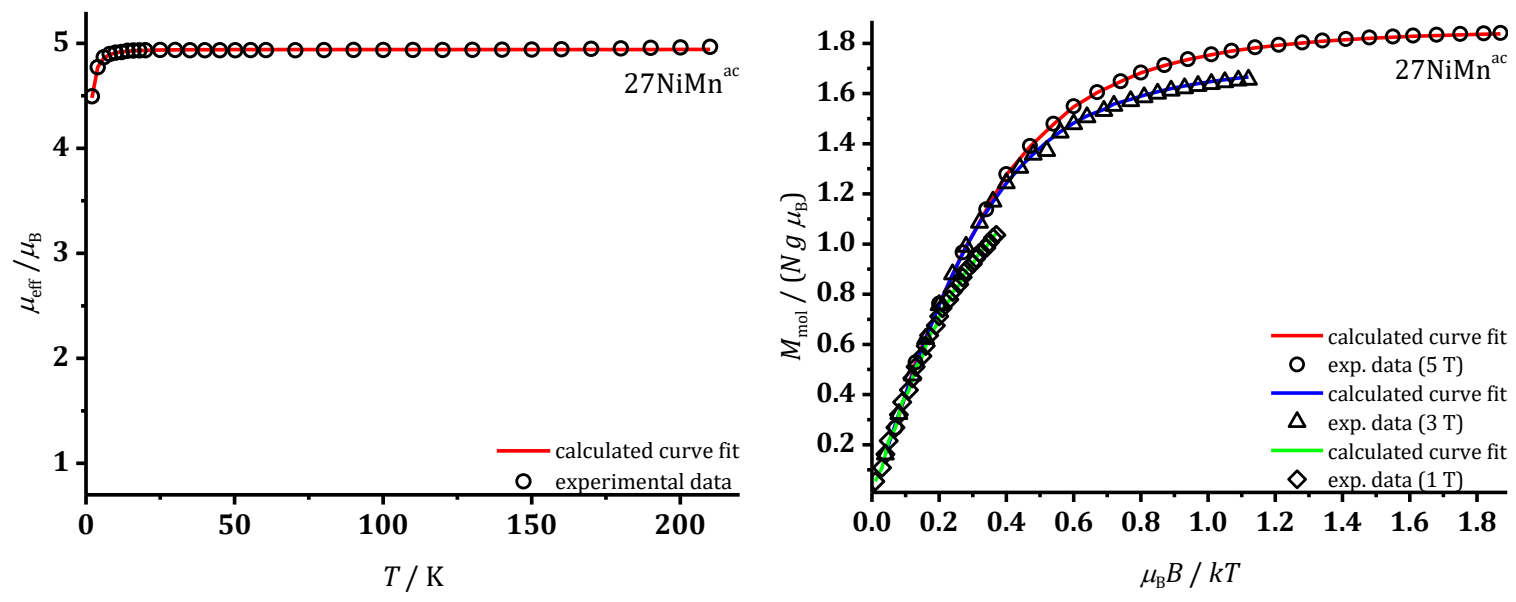

Figure 36: On the left, plot of $\mu_{\text {eff }}$ vs $T$ (2-295 K, solid sample) for nickel manganese complex 27 NiMn $^{\text {ac }}$ and the calculated curve fit (-). On the right, VTVH plot (2-50 K, solid sample) and calculated curve fits.

The separation of the first Kramer's doublet with $m_{S}= \pm 1$ and $m_{S}=0$ is described by the axial ZFS parameter $D$ and can be determined by the curve fit of experimental data as seen in Figure 36. The magnitude of the ZFS parameter with $D=+1.6 \mathrm{~cm}^{-1}$ (and $g=2.02$ ) is consistent with reported values from magnetic susceptibility measurements on Mn(III) porphyrin

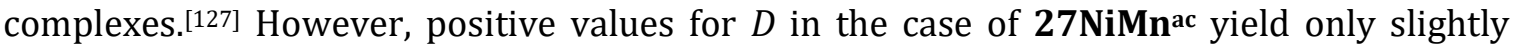
better fitting results than negative ones and further experiments such as high-field electron paramagnetic resonance spectroscopy (HF-EPR) have to be applied for confirmation.[128] Complementary HF-EPR experiments for $\mathbf{2 7 N i M n}{ }^{\text {ac }}$ in collaboration with Dr. EDWARD J. REIJERSE at the MPI for Chemical Energy Conversion, Mülheim an der Ruhr, as well as magnetic susceptibility measurements for complex $\mathbf{2 7} \mathbf{N i M n}^{\mathrm{az}}$ are still pending.

The slightly higher LANDÉ factor of $g=2.02$ for $\mathbf{2 7} \mathbf{N i M n}{ }^{\text {ac }}$ in comparison to the homobimetallic complexes $\mathbf{2 7} \mathbf{M n}_{2}{ }^{\text {ac }}$ and $\mathbf{2 7} \mathbf{M n}_{2}{ }^{\text {az }}$ is attributed to lower percentage of paramagnetic 
impurities and variant weighing errors that affect the fitting results. As complex 27NiMnac

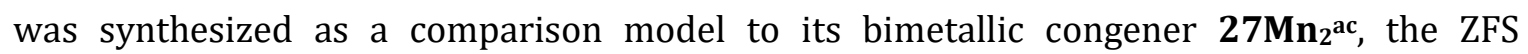
parameter with $\left(D=+1.6 \mathrm{~cm}^{-1}\right)$ determined from the VTVH measurement, was also included ex post into the curve fit for $\mathbf{2 7} \mathbf{M n}_{2}{ }^{\text {ac. }}$. However, no significant effects on the curve progression and the resulting parameters could be observed. The rather small ZFS is surpassed by the antiferromagnetic coupling and the resulting $S_{t}=0$ ground state at low temperatures.

\subsubsection{Cyclic Voltammetry Measurements}

Redox properties of $\mathbf{2 7} \mathbf{M n}_{2}{ }^{\text {ac }}$ and $\mathbf{2 7} \mathbf{M n}_{2}{ }^{\text {az }}$ were studied by cyclic voltammetry measurements (Figure 37) performed in $\mathrm{CH}_{2} \mathrm{Cl}_{2}$ with $\mathrm{Bu}_{4} \mathrm{NPF}_{6}(0.1 \mathrm{M})$ as the conducting salt. Decamethylferrocene $\left(\mathrm{FeCp}_{2}{ }_{2}\right)$ was added as an internal standard to avoid interference with compound signals. All redox potentials are reported with reference to the ferrocene/ferrocenium redox couple $\left(\mathrm{Fc} / \mathrm{Fc}^{+}\right)$with $E^{0}\left(\mathrm{FeCp}_{2}{ }_{2}\right)=-590 \mathrm{mV}$ vs $\mathrm{Fc} / \mathrm{Fc}^{+}$. All spectra were recorded at ambient temperature and under inert conditions.

Both complexes $\mathbf{2 7} \mathbf{M n}_{\mathbf{2}^{\mathrm{ac}}}$ and $\mathbf{2 7} \mathbf{M n}_{\mathbf{2}^{\mathrm{az}}}$ show poorly defined cyclic voltammograms with overlapping, irreversible redox waves. In the anodic section of the cyclic voltammograms three waves can be identified. The approximate location of the oxidations at $E_{1 / 2} \approx 0.00$ to $+0.74 \mathrm{~V}$ suggest a slight shift to higher redox potentials in $\mathbf{2 7} \mathbf{M n}_{2}{ }^{\text {ac }}$ and $\mathbf{2 7} \mathbf{M n}_{\mathbf{2}^{\mathbf{a z}}}$ in

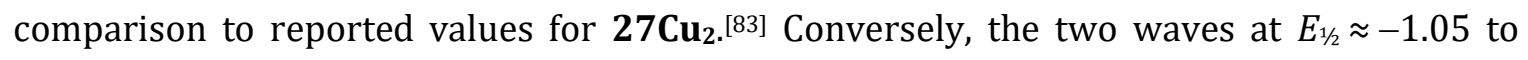
$-1.38 \mathrm{~V}$ indicate facilitated reduction of $\mathbf{2 7} \mathbf{M n}_{2^{\text {ac }}}$ and $\mathbf{2 7} \mathbf{M n}_{2^{a z}}$ compared to $\mathbf{2 7} \mathbf{C u}_{2}{ }^{\text {[83] }}$ The shift can be attributed to the higher initial charge of the central ion in the manganese(III) complexes and supports the proposed occurrence of a ligand-to-metal electron transfer as a basis for the expression of CR bands in the NIR region during UV/vis spectroscopic investigations (cf. Chapter 3.3.2). The poor resolution might result from the presence of several (interconverting) species in a variety of coordination geometries. ${ }^{[115,129]}$ Especially dissociation of the solvent molecule $(\mathrm{MeOH})$ to form pentacoordinate complexes is likely and redox-induced coordinational changes are common in manganese porphyrins. ${ }^{[115,129]}$

The lack of conclusive results from the redox analyses of complexes $\mathbf{2 7} \mathbf{M n}_{2}{ }^{\mathbf{a c}}$ and $\mathbf{2 7} \mathbf{M n _ { 2 }}{ }^{\mathrm{az}}$ in solution hampered any further investigations into the chemical redox chemistry of these species. Moreover, the similarity of the spectra of the manganese complexes among each
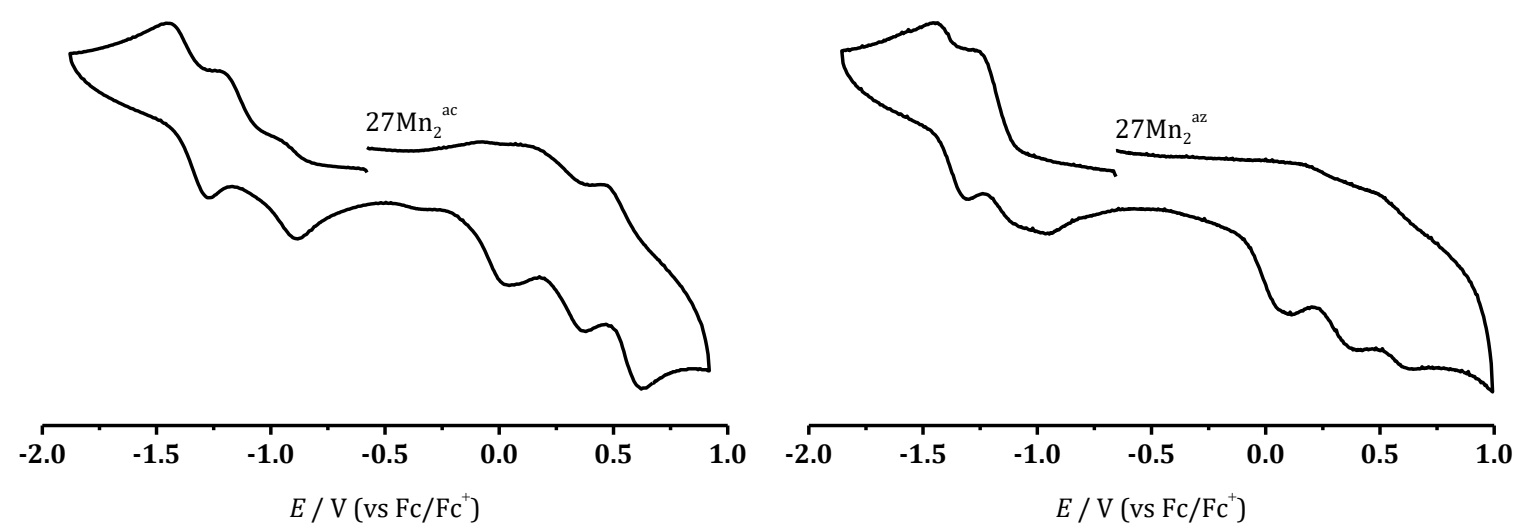

Figure 37: Cyclic voltammograms (vs Fc/Fc $)$ of the manganese complexes $\mathbf{2 7} \mathbf{M n}_{2} \mathbf{a c}^{\text {ac }}$ (left) and $\mathbf{2 7} \mathbf{M n}_{\mathbf{2}^{\mathbf{a z}}}$ (right) at room temperature in $\mathrm{CH}_{2} \mathrm{Cl}_{2}\left(0.1 \mathrm{M} \mathrm{Bu}_{4} \mathrm{NPF}_{6}, v=100 \mathrm{mVs}^{-1}\right)$. 
other and with known spectra of $\mathbf{2 7} \mathbf{C u}_{2}$ and $\mathbf{2 7} \mathbf{N i}_{2}$ did not provide any indication for accessibility of high-valent species via bulk redox reactions.

Comparative cyclic voltammetry studies for the heterobimetallic complexes $\mathbf{2 7} \mathbf{N i M n a c}$ and 27 NiMnaz are still pending.

\subsubsection{Photolysis}

Azido manganese complexes are known to undergo oxidative elimination of dinitrogen upon photochemical activation yielding high-valent manganese nitrido complexes. ${ }^{[23,25,26]}$ Thus, a solution of $\mathbf{2 7} \mathbf{M n}_{2}{ }^{\text {az }}$ in toluene was irradiated with a high-power UV lamp (broadband) and the reaction progress was monitored by UV/vis spectroscopy. Unfortunately, only bleaching due to substrate decomposition could be detected. Additional cooling of the sample solution and irradiation at selected wavelengths (e.g. $\lambda=365 \mathrm{~nm}$ ) to minimize substrate decomposition did not lead to any improvements.

Due to the discouraging results in solution chemistry of $\mathbf{2 7} \mathbf{M n}_{\mathbf{2}} \mathbf{a z}$, a solid-state approach for the photochemical activation (high-power UV lamp, optical filter for $\lambda=280 \mathrm{~nm}$ ) was chosen. Due to the prominent and isolated band of $\tilde{v}=2040 \mathrm{~cm}^{-1}$ for the asymmetric stretch of metalbound azide in $\mathbf{2 7} \mathbf{M n}_{\mathbf{2}^{\mathrm{az}}}$, IR spectroscopy ( $\mathrm{KBr}$ pellet) was chosen to monitor the irradiation progress (Figure 38).

Upon irradiation of $\mathbf{2 7} \mathbf{M n}_{2}{ }^{\mathrm{az}}$, gradual depletion of the peak at $\tilde{v}=2040 \mathrm{~cm}^{-1}$ can be observed. Due to increasing opacity of the sample under exposure to air during the measurement, excessive baseline corrections had to be applied. Nonetheless, signals not related to the $\mathrm{M}-\mathrm{N}_{3}$ moiety showed no changes at all, verifying so the significance of the results. Unfortunately, no new bands indicative of the formation of a $\mathrm{Mn} \equiv \mathrm{N}$ unit could be detected. The nitrido manganese stretching vibration is expected at $\tilde{v} \approx 1040 \mathrm{~cm}^{-1}$ as a weak band.[23,25] However, this section is showing an intense signal pattern already for the starting material $\mathbf{2 7} \mathbf{M n}_{\mathbf{2}} \mathbf{a z}^{\mathbf{a}}$ thus impeding the monitoring of potential intensity changes upon formation of $\mathrm{Mn} \equiv \mathrm{N}$.

The lack of pronounced changes upon extensive irradiation with a high-power UV-source may be attributed to the highly colored and thereby strongly absorbing macrocyclic chromophore (cf. Chapter 3.3.2). Thus, the efficiency of the required excitation of the nitrido unit is impaired by the extended $\pi$-conjugated system and therewith associated absorptions in the UV region.
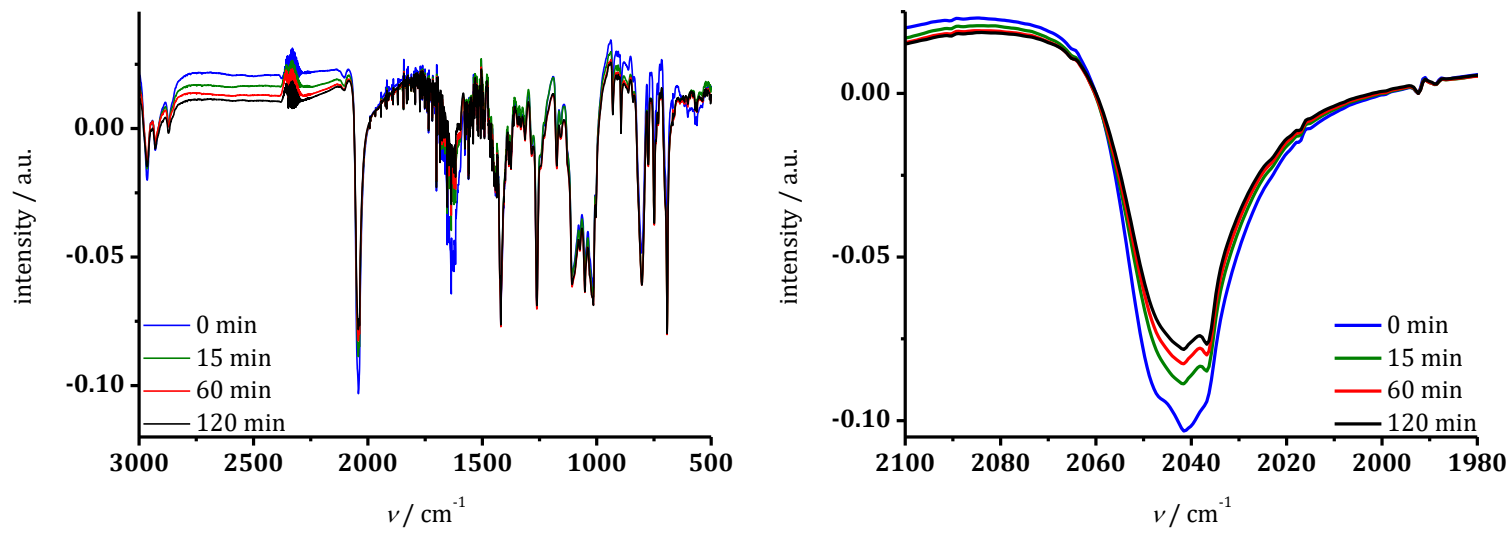

Figure 38: Progression of IR spectra during UV-irradiation of $\mathbf{2 7} \mathbf{M n}_{2} \mathbf{2}^{\mathbf{a z}}$ (right) and section of the spectrum with relevant asymmetric stretching vibration at $\tilde{\boldsymbol{v}}=2040 \mathrm{~cm}^{-1}$ (right). 
Alternative activation of azido complexes towards the formation of a $\mathrm{Mn} \equiv \mathrm{N}$ unit involves thermolysis in high-boiling solvents over a long period of time. ${ }^{[25]}$ However, in case of the Siamese-twin porphyrin complex $\mathbf{2 7} \mathbf{M n}_{2}{ }^{\text {az }}$ gradual decomposition of the ligand sets in within $30 \mathrm{~min}$ of the reaction start and before any indication for formation of high-valent $\mathrm{Mn} \equiv \mathrm{N}$ species.

Further efforts towards the formation of high-valent species from complexes of the Siamesetwin porphyrin $\mathbf{2 7} \mathbf{H}_{4}$ could include elimination of an axial ligand to form pentacoordinated complexes with an open coordination site (e.g. use of non-coordinating counterions such as $\mathrm{ClO}_{4}^{-}$). Such complexes are known to form nitrido species upon treatment with hypochlorite in the presence of ammonia,[130] iodosobenzene (PhIO) in the presence of $\mathrm{NH}_{4} \mathrm{Cl}^{,[131]}$ or application of a nitrogen transfer reagent.[132] Furthermore, complexes 27NiMnac and 27 NiMnaz should be included into further studies to facilitate interpretation of spectra and identification of products. 


\subsection{Palladium Complexes}

Palladium coordination was shown to induce topological reorganizations in large and flexible expanded porphyrins (Chapter 1.3.4).[72] However, no $4 \mathrm{~d}$ metal ions have yet been introduced into the square-planarly preorganized $\left\{\mathrm{N}_{4}\right\}$-coordination pockets of the Siamese-twin porphyrin $\mathbf{2 7} \mathbf{H}_{4}$. Previous reports on nickel mono- and bimetalation $\left(\mathbf{2 7} \mathbf{H}_{2} \mathbf{N i}\right.$ and $\left.\mathbf{2 7} \mathbf{N i}_{2}\right)$, nickel being the lighter congener of palladium, demonstrated the general coordination chemistry and offered first insights into the redox chemistry of such a system. ${ }^{\left[{ }^{[2]}\right.}$ In many aspects of the following chapters comparisons with the reported nickel complexes ${ }^{[76,82,84]}$ and the free-base Siamese-twin porphyrin[76,84] (also Chapters 1.3.5, 1.3.6 and 3.1) will be drawn. The results presented in the following chapters were summarized in a publication.[133]

\subsubsection{Siamese-Twin Porphyrin Metalation}

Complexation of the Siamese-twin porphyrin $\mathbf{2 7} \mathbf{H}_{\mathbf{4}}$ with palladium(II) salts (Scheme 18) was carried out similarly to the procedure reported for the synthesis of manganese(III) compounds (Chapter 3.3.1). Acetate metal salts are frequently the reactant of choice for the metalation of polypyrrolic macrocyles.[134] However, it proved ineffective in case of the Siamese-twin porphyrin $\mathbf{2} \mathbf{2 H}_{4}$ and thus $\mathrm{Pd}(\mathrm{acac})_{2}$ was used instead. The acetylacetonate anion $\left(\mathrm{acac}^{-}\right)$makes the selected salt satisfactory soluble in the reaction solvent and, at the same time, it serves as a base for released protons during the reaction.

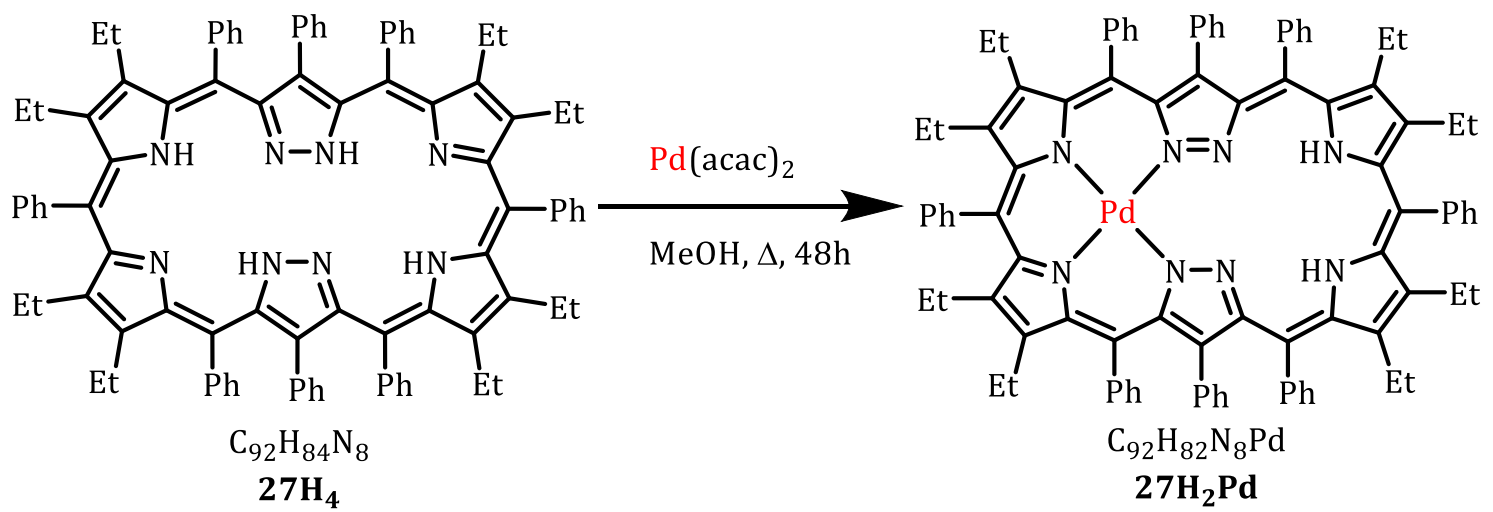

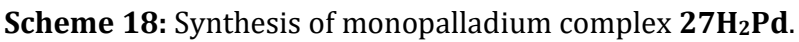

Reaction of blue-green Siamese-twin porphyrin $\mathbf{2} \mathbf{7} \mathbf{H}_{4}$ with six equivalents of $\mathrm{Pd}(\mathrm{acac})_{2}$ under inert conditions over two days at reflux in methanol led to an olive-green colored precipitate. TLC indicated the consumption of the free-base ligand $\mathbf{2 7} \mathbf{H}_{\mathbf{4}}$ and mass spectrometry showed the formation of a single new product. Product $\mathbf{2 7} \mathbf{H}_{2} \mathbf{P d}$ was isolated in $43 \%$ yield by filtration and subsequent purification by column chromatography (neutral alumina, $n$-hexane/THF/EtOAc 20:3:1, $\left.R_{\mathrm{f}}\left(\mathbf{2 7}_{2} \mathbf{P d}\right)=0.50\right)$. The composition, as determined by HR-MS $\left(\mathrm{ESI}^{+}\right)$, suggested that $\mathbf{2 7} \mathbf{H}_{2} \mathbf{P d}$ is a monopalladium complex of $\mathbf{2 7} \mathbf{H}_{4}\left(\mathrm{C}_{92} \mathrm{H}_{83} \mathrm{~N}_{8} \mathrm{Pd}^{+}\right.$for $\mathrm{MH}^{+}$; Figure 39). A minor amount $(\sim 5 \%)$ of a dipalladium species $\mathbf{2 7} \mathbf{P d}_{2}$ appeared untraceable during the described purification process and was detected firstly after crystallization of $\mathbf{2 7} \mathbf{H}_{\mathbf{2}} \mathbf{P d}$ (cf. Chapter 3.4.3). The composition of byproduct $\mathbf{2 7} \mathbf{P \mathbf { d } _ { 2 }}$ was confirmed by HR-MS $\left(\mathrm{C}_{92} \mathrm{H}_{80} \mathrm{~N}_{8} \mathrm{Pd}_{2}{ }^{+}\right.$for $M^{+}$; Figure 40$)$. 
All attempts to selectively synthesize $\mathbf{2 7 P d _ { 2 }}$ were unsuccessful so far. This comes as no surprise considering the difference in synthetic conditions for $\mathbf{2 7} \mathbf{N i}_{2}$ in comparison to $\mathbf{2 7}_{\mathbf{2}} \mathbf{N i}$ :[82] while monometalation occurs within $15 \mathrm{~min}$ at room temperature, refluxing conditions over a period of $24 \mathrm{~h}$ have to be applied to yield coordination of the second metal ion. Thus, extrapolating towards $\mathbf{2 7} \mathbf{H}_{2} \mathbf{P d}$, dipalladium complex $\mathbf{2 7} \mathbf{P d}_{2}$ seems not quantitatively accessible prior to any decomposition of the free-base ligand $\mathbf{2 7} \mathbf{H}_{\mathbf{4}}$ at such harsh reaction conditions. Therefore, any further analyses of $\mathbf{2 7} \mathbf{P} \mathbf{d}_{2}$ had to be renounced.
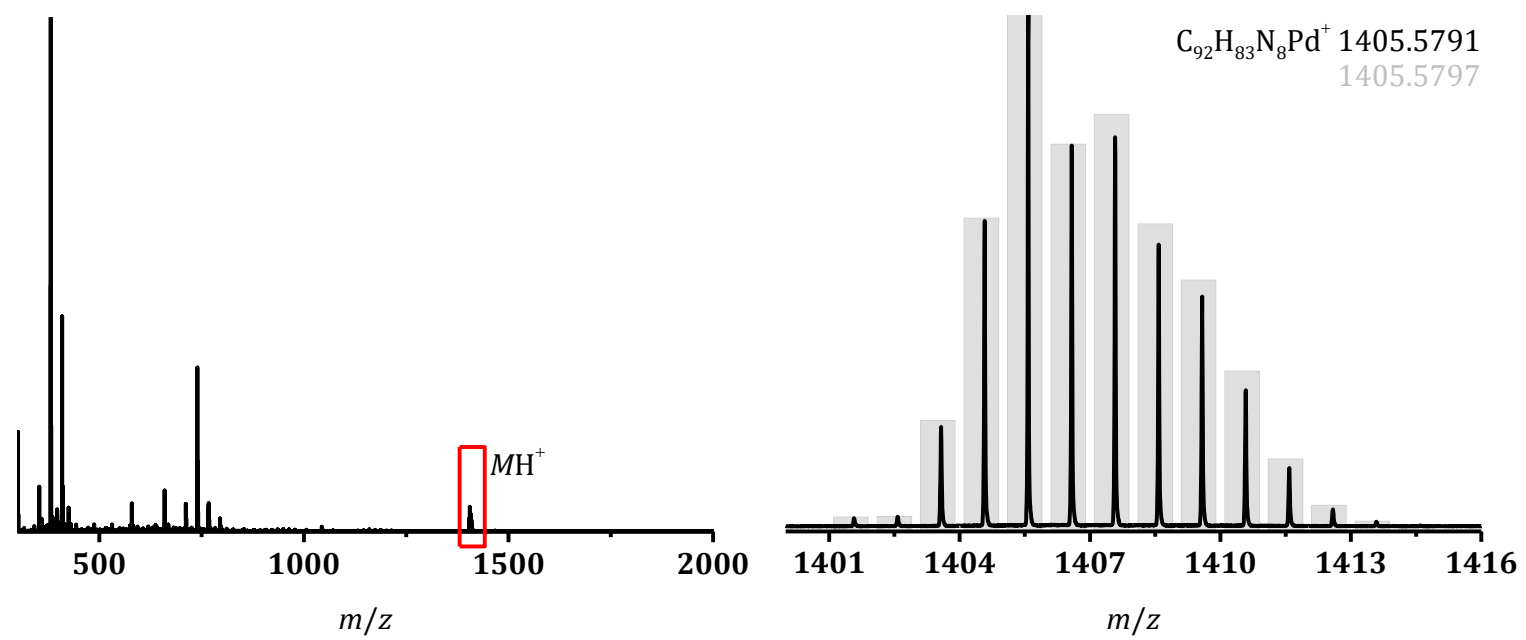

Figure 39: $\mathrm{HR}^{-\mathrm{ESI}^{+}}$spectrum of $\mathbf{2 7} \mathbf{H}_{2} \mathbf{P d}$ (highlighted in red) and the isotopic distribution of the molecular peak $M \mathrm{H}^{+}$(simulation in grey).
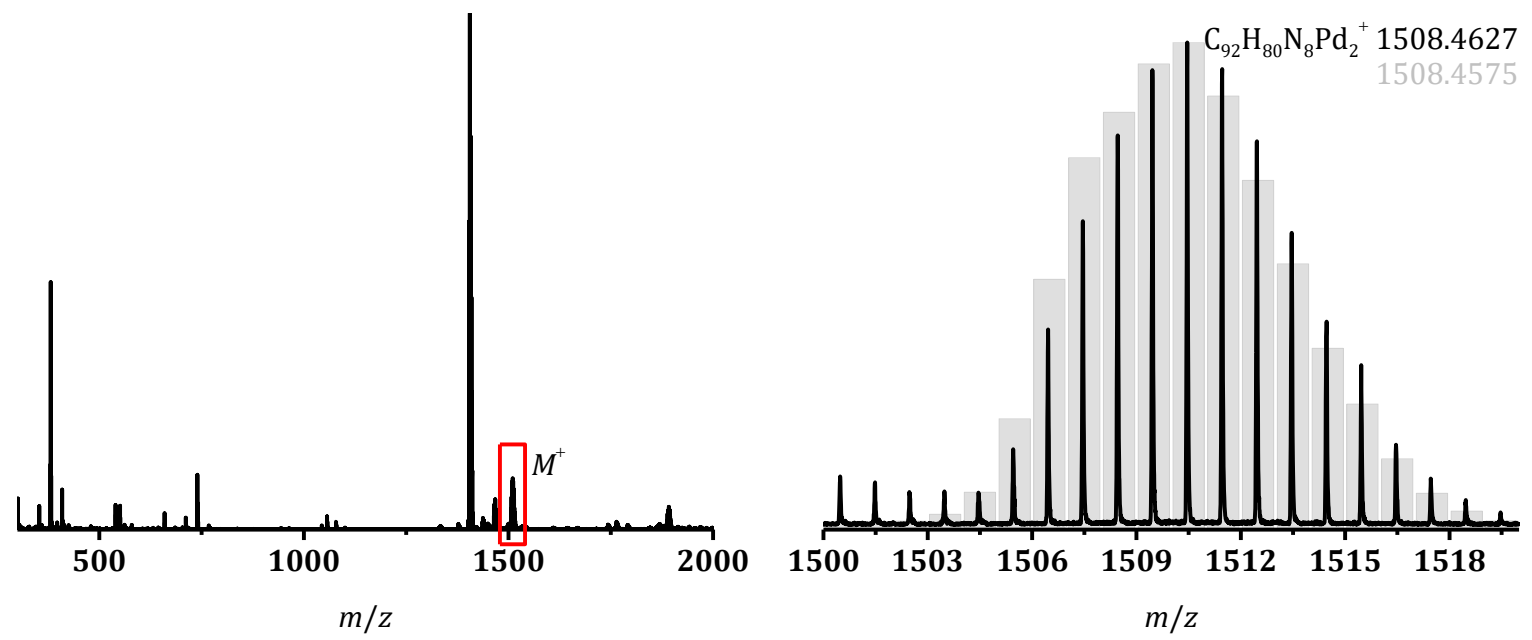

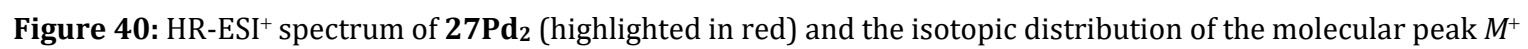
(simulation in grey).

\subsubsection{UV/vis Spectral Characterization}

The UV/vis spectrum of the metalation product $\mathbf{2 7} \mathbf{H}_{2} \mathbf{P d}$ (Figure 41, left) shows a significant red-shift $(\Delta \lambda=20-59 \mathrm{~nm})$ in all prominent features relative to the spectrum of the Siamesetwin porphyrin $\mathbf{2 7 \mathbf { H } _ { 4 }}$ (Table 8). While the SORET-like band $(\lambda=409 \mathrm{~nm})$ forfeits about a quarter of its intensity upon metalation, the Q-like band $(\lambda=662 \mathrm{~nm})$ nearly doubles in intensity. This results in a unique 1:1-ratio in intensities of both bands in $\mathbf{2 7} \mathbf{H}_{\mathbf{2}} \mathbf{P d}$ that is unique among all previously reported Siamese-twin porphyrin metal complexes. ${ }^{276,82,83]}$ This 

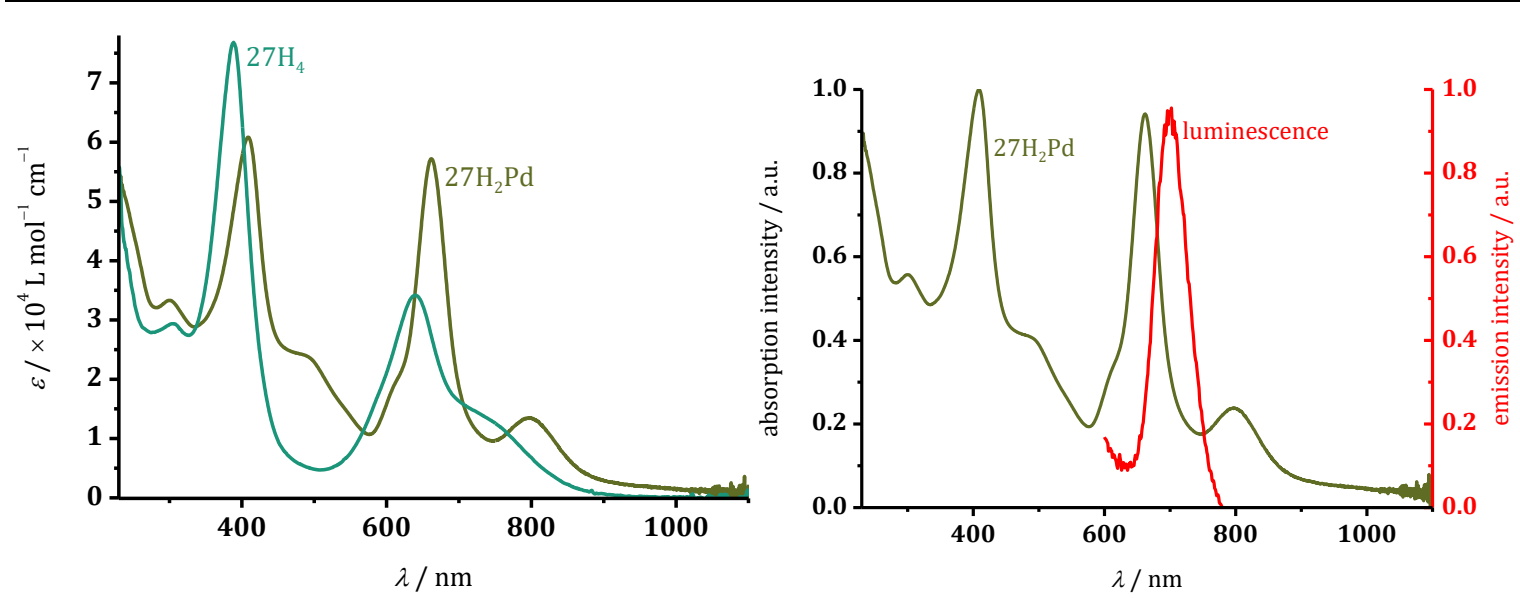

Figure 41: On the left, UV/vis spectra ( $298 \mathrm{~K}_{2} \mathrm{CH}_{2} \mathrm{Cl}_{2}$ ) of monopalladium complex $\mathbf{2 7} \mathbf{H}_{2} \mathbf{P d}$ (left, olive-green trace) and the free-base ligand $\mathbf{2} \mathbf{7 H}_{\mathbf{4}}$ (teal trace) for comparison. On the right, normalized UV/vis absorption (olive-green trace) and fluorescence emission (red trace) spectra (298 K, $\mathrm{CH}_{2} \mathrm{Cl}_{2}, \lambda_{\text {ex }}=410 \mathrm{~nm}$ ) of complex $\mathbf{2 7} \mathbf{H}_{2} \mathbf{P d}$.

trend is matched solely by two-fold protonation of the free-base Siamese-twin porphyrin $\mathbf{2 7 H}_{4}$ (cf. Figure 7).[76]

The essential mechanism for these spectroscopic changes in both the complexation with palladium $\left(\mathbf{2 7} \mathbf{H}_{2} \mathbf{P d}\right)$ and the protonation of the neutral free-base ligand $\left(\mathbf{2 7 H}_{\mathbf{6}} \mathbf{2}^{\mathbf{2}}\right)$ is the gradual coplanarization of the pyrrole and pyrazole moieties and thus abolishment of the high distortion on all meso-carbon atoms of the macrocycle (cf. Figure 13). Moreover, the enforced square-planar arrangement of the involved nitrogen atoms locks the entire macrocyclic backbone in a distinct conformation, consequently minimizing molecular movement and leading to better-resolved spectroscopic transitions. This claim is further supported by NMR and X-ray crystallographic molecular structure analyses presented further below.

Table 8: UV/vis spectral data* of free-base ligand $27 \mathbf{H}_{4}$, and palladium complexes $2 \mathbf{2 7} \mathbf{H}_{2} \mathbf{P d}, \mathbf{2 7} \mathbf{H}_{3} \mathbf{P d} \mathbf{d}^{+}$, and

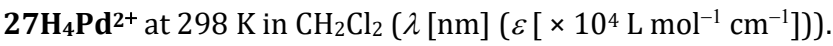

\begin{tabular}{|c|c|c|c|}
\hline $27 \mathrm{H}_{4}$ & $27 \mathrm{H}_{2} \mathrm{Pd}$ & $27 \mathrm{H}_{3} \mathrm{Pd}^{+}$ & $27 \mathrm{H}_{4} \mathrm{Pd}^{2+}$ \\
\hline 305 (2.9) & $300(3.3)$ & $296(3.4)$ & $296(3.4)$ \\
\hline 389 (7.7) & $408(6.1)$ & $398(6.6)$ & $381(7.6)$ \\
\hline- & $477(2.4)$ & $469(2.5)$ & - \\
\hline - & $610(1.8)$ & $589(1.6)$ & $583(1.8)$ \\
\hline $641(3.4)$ & $662(5.7)$ & $644(5.5)$ & $634(5.9)$ \\
\hline $740(1.3)$ & 799 (1.4) & 767 (1.4) & $768(2.0)$ \\
\hline
\end{tabular}

* Isosbestic points for first protonation step: $\lambda=273,303,349,407,450,474,574,654,713$ and $790 \mathrm{~nm}$; and for second protonation step: $\lambda=333,391,550,640,698$ and $811 \mathrm{~nm}$.

As elaborated in the introduction, metalation is expected to rigidify the otherwise flexible scaffold of expanded porphyrins and may hence promote luminescent behavior of the respective systems. Thus, luminescence (Figure 41, right) of the monopalladium complex $\mathbf{2 7} \mathbf{H}_{2} \mathbf{P d}$ was probed. While the free-base ligand $\mathbf{2 7} \mathbf{H}_{\mathbf{4}}$ shows no emission at all, excitation of $\mathbf{2 7} \mathbf{H}_{2} \mathbf{P d}$ at $\lambda_{\mathrm{ex}}=410 \mathrm{~nm}\left(298 \mathrm{~K}, \mathrm{CH}_{2} \mathrm{Cl}_{2}\right)$ showed very weak emission at $\lambda=699 \mathrm{~nm}$. Cooling 
to $77 \mathrm{~K}$ to prevent non-radiative decay did not lead to any significant increase in the emission intensity.

Monometalation of the Siamese-twin porphyrin inevitably leads to a complex with an adjacent, vacant coordination site that is assumingly $\mathrm{pH}$-sensitive. Acid/base titrations were performed to probe the protonation behavior of complex $\mathbf{2 7} \mathbf{H}_{2} \mathbf{P d}$ and UV/vis (Figure 42 , Table 8) and NMR spectroscopy (Chapter 3.4.3) were chosen for monitoring.

Monopalladium complex $\mathbf{2 7} \mathbf{H}_{2} \mathbf{P d}$ was titrated with a $0.1 \mathrm{M}$ stock solution of trifluoroacetic acid (TFA) or 1,8-diazabicyclo[5.4.0] undec-7-en (DBU) in $\mathrm{CH}_{2} \mathrm{Cl}_{2}$ in 0.1 eq steps. Upon addition of TFA, a blue-shift $(\Delta \lambda=10-32 \mathrm{~nm}$ for the first equivalent and $\Delta \lambda=10-17 \mathrm{~nm}$ for the second equivalent) of all prominent peaks could be observed. Further, the shoulder at $\lambda=477 \mathrm{~nm}$ and the band at $\lambda=799 \mathrm{~nm}$ proved to be distinctly sensitive to the acid/base titration suggesting a significant contribution of the vacant, $\mathrm{pH}$-responsive coordination pocket to the given transitions. This observation is further supported by redox studies and therewith associated computations (Chapter 3.4.5) discussed further below.

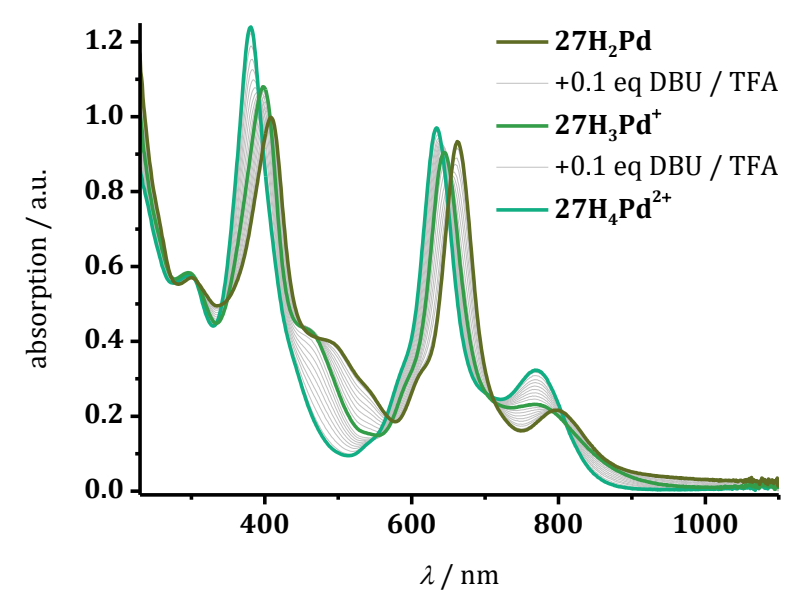

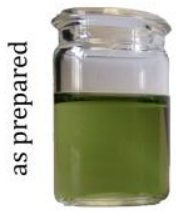
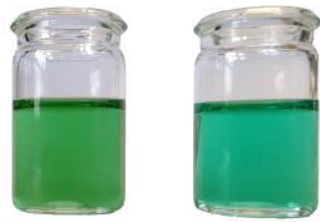

$27 \mathrm{H}_{2} \mathrm{Pd}$
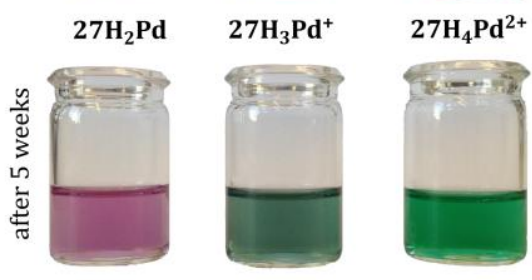

Figure 42: Acid/base titration followed by UV/vis $\left(298 \mathrm{~K}, \mathrm{CH}_{2} \mathrm{Cl}_{2}\right)$ of monopalladium complex $\mathbf{2 7} \mathbf{H}_{2} \mathbf{P d}$ (olive trace). Mono- $\left(\mathbf{2 7} \mathbf{H}_{3} \mathbf{P d}^{+}\right.$, green trace) and diprotonated species $\left(\mathbf{2 7} \mathbf{H}_{4} \mathbf{P d} \mathbf{d}^{+}\right.$, teal trace) were detected upon addition of one and two equivalents of acid (TFA), respectively. All spectral changes are fully reversible and show isosbestic points for each transition. Further addition of acid/base did not lead to any spectral changes. Decomposition occurs in all samples starting within days for $\mathbf{2 7} \mathbf{H}_{2} \mathbf{P d}$ and weeks for $\mathbf{2 7} \mathbf{H}_{4} \mathbf{P d}^{2+}$ (right, bottom row).

Coherent changes in the UV/vis spectrum with clear isosbestic points (Table 8) were observed until the addition of one equivalent (formation of $\mathbf{2 7} \mathbf{H}_{3} \mathbf{P d}^{+}$) and further until the addition of two equivalents of TFA (formation of $\mathbf{2 7} \mathbf{H}_{4} \mathbf{P d} \mathbf{2}^{2+}$ ). No further changes could be detected upon addition of a third and fourth equivalent. These findings are consistent with a dibasic, vacant coordination pocket in complex $\mathbf{2 7} \mathbf{H}_{2} \mathbf{P d}$ in its neutral form. The presence of clear isosbestic points shows a clean interconversion of the species with no decomposition of the complex. Further structural evidence is given by the NMR spectroscopic characterization (Chapter 3.4.3) of the neutral as well as the mono- and diprotonated species.

All spectroscopic changes are fully reversible and titration of the diprotonated species $\mathbf{2 7} \mathbf{H}_{4} \mathbf{P d}^{2+}$ with a $0.1 \mathrm{M}$ stock solution of DBU in $\mathrm{CH}_{2} \mathrm{Cl}_{2}$ in 0.1 eq steps led initially to full recovery of the spectrum of $\mathbf{2 7} \mathbf{H}_{3} \mathbf{P d}^{+}$and subsequently of $\mathbf{2} \mathbf{7} \mathbf{H}_{2} \mathbf{P d}$. Full recovery of the initial spectrum of $\mathbf{2 7} \mathbf{H}_{2} \mathbf{P d}$ once again underlines the stability of the palladium coordination towards stoichiometric amounts of acid and excludes the possibility of decomposition during 
the titration as cause for the spectral changes. No further deprotonation of $\mathbf{2 7} \mathbf{H}_{2} \mathbf{P d}$ could be achieved by addition of DBU according to the UV/vis spectra.

For all species, decomposition in solution $\left(\mathrm{CH}_{2} \mathrm{Cl}_{2}\right.$; $\mathrm{cf}$. Figure 42$)$ sets in within days $\left(\mathbf{2 7} \mathbf{H}_{2} \mathbf{P d}\right)$ up to weeks $\left(\mathbf{2 7} \mathbf{H}_{4} \mathbf{P d}^{2+}\right)$ under aerobic conditions. The higher stability of the protonated complexes may be attributed to the enhanced rigidity upon protonation as well as solvent and counterion stabilizing effects through electrostatic interactions.

In comparison to the protonation studies of $\mathbf{2 7} \mathbf{H}_{2} \mathbf{N i}$ performed by BLUSCH, ${ }^{[84]}$ monopalladium complex $\mathbf{2 7} \mathbf{H}_{2} \mathbf{P d}$ offers an increased stability and therefore more accurate results. While the $\mathrm{UV} /$ vis and NMR spectroscopic investigations of $\mathbf{2 7} \mathbf{H}_{2} \mathbf{N i}$ were contradictive and troubled by decomposition, ${ }^{[84]} \mathbf{2 7} \mathbf{H}_{2} \mathbf{P d}$ offered, as detailed above, highly reproducible and consistent data.

\subsubsection{X-ray Crystallographic Molecular Structures}

Single crystals of the monopalladium complex $\mathbf{2 7} \mathbf{H}_{2} \mathbf{P d}$ suitable for X-ray diffraction were obtained by layering a saturated solution of $\mathbf{2 7} \mathbf{H}_{2} \mathbf{P d}$ in $\mathrm{THF}$ with $\mathrm{MeOH}$. The data were resolved in the triclinic, non-chiral space group $P \overline{1}$, showing the presence of a racemic mixture of two helimeric conformers of $\mathbf{2 7} \mathbf{H}_{2} \mathbf{P d}$ (Figure 43; Chapter 6.1). As expected from previously described analyses, structural refinement showed the known Siamese-twin porphyrin scaffold and one palladium ion in the first coordination pocket. The lack of any counterions and the square-planar coordination of the palladium ion point to an oxidation state of +II for the metal ion. The quality of the diffraction data allowed further the localization of two hydrogen atoms in the second, metal-free coordination site. Both hydrogen atoms were identified on the dipyrrin unit engaged in a hydrogen bond network with proximal solvent molecules $(\mathrm{MeOH})$. Hence, the pyrazole nitrogen atoms on both long sides of the molecule appear imine-like-a result of a palladium-induced NH-tautomerism. Coordination of the LEWIS acidic palladium ion in one coordination pocket withdraws electron density from the pyrazole, therefore acidifying the pyrazole-bound proton and enforcing its shift onto the adjacent pyrrolic subunit. This phenomenon was also observed by BLUSCH for the X-ray crystallographic molecular structure (Figure 8) and associated DFT calculations of $\mathbf{2 7} \mathbf{H}_{2} \mathbf{N i}^{\text {. }}{ }^{82}$ ]

In contrast to the less-favored cis-NH-configuration in porphin (by $7.6 \mathrm{kcal} / \mathrm{mol}$ ), ${ }^{[135]}$ the inherent twist of the Siamese-twin porphyrin $\mathbf{2 7} \mathbf{H}_{4}$ renders it particularly suitable for localizing two protons on adjacent subunits. The up-and-down orientation of the pyrrole and pyrazole nitrogen atoms induced by the macrocyclic distortion (Figure 43C) prevents any build-up of strain in the first place. Thus, no additional distortion contribution from the proton shift is evident in the structural parameters (cf. Chapter 6.1). The cis-configuration of $\mathrm{NH}-$ moieties in the vacant coordination pocket results in a (non-crystallographic) pseudo- $C_{2}-$ symmetry for $\mathbf{2 7} \mathbf{H}_{2} \mathbf{P d}$.

The twist of the macrocyclic backbone in the monopalladium complex $27 \mathbf{H}_{2} \mathbf{P d}(\tau= \pm 84$ / $\pm 93^{\circ}$; Table 9 ) is slightly more relaxed when compared to the crystal structure of the freebase Siamese-twin porphyrin $\mathbf{2 7 \mathbf { H } _ { 4 }}\left(\tau= \pm 97^{\circ}\right)$. This comes as no surprise considering the planarization enforced by the preferably square-planar coordination environment of $\mathrm{Pd}(\mathrm{II})$. However, coordination geometry alone cannot explain the relaxation of the macrocyclic backbone when $\mathbf{2} \mathbf{7 H}_{2} \mathbf{P d}$ and $\mathbf{2} \mathbf{7} \mathbf{H}_{2} \mathbf{N i}$ are compared. Additionally, the size of the metal ion 
itself $(r(\mathrm{Pd}(\mathrm{II}))=78 \mathrm{pm} \text { vs } r(\mathrm{Ni}(\mathrm{II}))=63 \mathrm{pm} \text { in a square-planar coordination })^{[136]}$ plays a significant role. While the size of the palladium ion allows to keep the coordinating moieties at a distance comparable with the void in the metal-free ligand $\left(d_{\text {trans }}(\mathrm{N} \cdots \mathrm{N})=3.98 \AA\right.$ for $\mathbf{2 7} \mathbf{H}_{2} \mathbf{P d}$ vs $d_{\text {trans }}(\mathrm{N} \cdots \mathrm{N})=4.09-4.35 \AA$ for $\left.\mathbf{2 7} \mathbf{H}_{6}{ }^{2+}\right),{ }^{+76]}$ the smaller nickel ion pulls the coordinating groups closer $\left(d_{\text {trans }}(\mathrm{N} \cdots \mathrm{N})=3.70 \AA\right.$ for $\left.\mathbf{2 7} \mathbf{H}_{2} \mathbf{N i}\right),{ }^{[82]}$ distorting thus the macrocycle and leading to a higher overall twist $\left(\tau= \pm 92 / \pm 99^{\circ}\right)$.

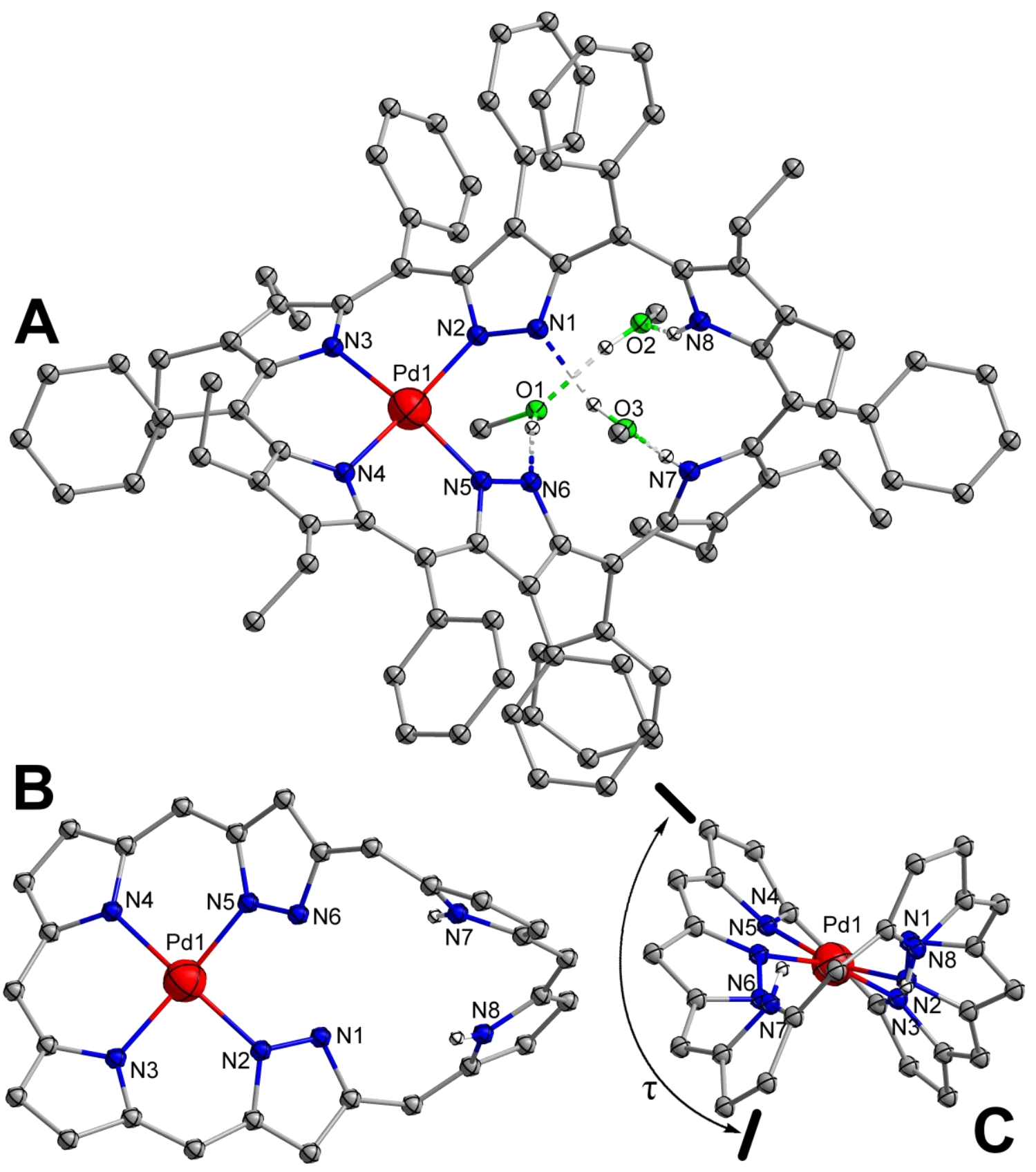

Figure 43: Ball-and-stick representation of the solid state structure of the monopalladium complex of the Siamese-twin porphyrin $\mathbf{2 7} \mathbf{H}_{2} \mathbf{P d}$ (palladium in red, oxygen in green, carbon in grey, nitrogen in blue, and hydrogen in white). Only one enantiomer shown; solvent molecules (except in A) and carbon-bound hydrogen atoms omitted for clarity. A) Overview including peripheral substituents; B) representation of macrocycle conformation highlighting cis-arrangement of $\mathrm{NH}$ groups, and $\mathrm{C}$ ) view along the long axis depicting the torsion angle $\tau$. 
Table 9: Structural parameters for $27 \mathbf{H}_{4}, \mathbf{2 7} \mathbf{H}_{2} \mathbf{P d}, \mathbf{2 7} \mathbf{H}_{2} \mathbf{N i}, 27 \mathbf{P d}_{2}$ and $27 \mathbf{N i}_{2}$.

\begin{tabular}{|c|c|c|c|c|c|}
\hline & $27 \mathrm{H}_{4}$ & $27 \mathrm{H}_{2} \mathrm{Pd}$ & $27 \mathrm{H}_{2} \mathrm{Ni}^{\mathrm{a}}$ & $27 \mathrm{Pd}_{2}$ & $27 \mathrm{Ni}_{2}{ }^{\mathrm{a}}$ \\
\hline$\tau\left[^{\circ}\right]$ & \pm 97 & $\pm 85 / \pm 93$ & $\pm 92 / \pm 99$ & \pm 94 & $\pm 104 / \pm 110$ \\
\hline$\left.d_{\text {helix }}[\AA]\right]$ & 10.78 & 10.81 & 10.65 & 10.62 & 10.48 \\
\hline$d_{\text {trans }}(\mathrm{N} \cdots \mathrm{N})[\AA]$ & -b & 3.98 & 3.70 & 4.04 & 3.79 \\
\hline$d_{\min }(\mathrm{C}-\mathrm{C})[\AA]$ & 1.36 & 1.36 & 1.36 & 1.36 & 1.36 \\
\hline$d_{\max }(\mathrm{C}-\mathrm{C})[\AA]$ & 1.48 & 1.47 & 1.47 & 1.48 & 1.47 \\
\hline$d(\mathrm{M} \cdots \mathrm{M})[\AA]$ & - & - & - & 3.93 & 3.81 \\
\hline$d(\mathrm{Mn}-\mathrm{N})[\AA \AA]$ & - & $1.99-2.02$ & $1.87-1.88$ & $2.02-2.04$ & $1.90-1.92$ \\
\hline$\Varangle(\mathrm{NMN})\left[^{\circ}\right]$ & - & 93 / 89 / 94 / 88 & 91 / 94 / 91 / 93 & 92 / 89 / 91 / 90 & $91 / 91 / 91 / 90$ \\
\hline
\end{tabular}

a Measurements were performed on structures deposited at the Cambridge Crystallographic Data Center: CCDC 1020365 and 906533.[82] b The measurement was omitted due to the lack of a square-planar arrangement of the nitrogen atoms caused by the severe twist of the macrocyclic backbone (cf. Figure 13).

The alternating short-long pattern in bond lengths of the two individual conjugation pathways in $\mathbf{2} \mathbf{7 H}_{\mathbf{2}} \mathbf{P d}$ differs slightly from the free-base ligand $\mathbf{2} \mathbf{7} \mathbf{H}_{\mathbf{4}}$ (cf. Chapter 6.1). The most remarkable difference is represented in the dipyrrin subunit that experiences an averaging in bond lengths that is even more pronounced than in the cases of $\mathbf{2 7} \mathbf{M} \mathbf{n}_{\mathbf{z}^{\mathrm{ac}}}$ and $\mathbf{2 7} \mathbf{\mathbf { M n }} \mathbf{2}^{\mathrm{az}}$ (cf. Chapter 3.3.3).

Crystallographic data of $\mathbf{2 7} \mathbf{H}_{2} \mathbf{P d}$ revealed further the presence of a dipalladium species 27Pd 2 . The complex was taken into account during the refinement as a disorder with an occupancy of $5 \%$. Repeated subjection of the mother liquor from $\mathbf{2 7} \mathbf{H}_{2} \mathbf{P d}$ to altered crystallization conditions yielded an alternate batch of crystals and thus confirmed the presence of the previously undetected complex $\mathbf{2 7} \mathbf{P} \mathbf{d}_{2}$.

Single crystals of the dipalladium complex $\mathbf{2 7} \mathbf{P d}_{2}$ suitable for X-ray diffraction were obtained by recovery of mother liquor residue from crystallization of $\mathbf{2 7} \mathbf{H}_{2} \mathbf{P d}$ through evaporation of solvent in vacuo, take-up in minimal amounts of $\mathrm{CH}_{2} \mathrm{Cl}_{2}$ and layering with $\mathrm{MeOH}$. The data were resolved in the monoclinic, non-chiral space group $C 2 / c$, showing the presence of a racemic mixture of two helimeric conformers of $\mathbf{2 7} \mathbf{P d}_{\mathbf{2}}$ (Figure 44, Chapter 6.1) of $\mathrm{C}_{2}$ symmetry. Structural refinement revealed the macrocyclic motif of the Siamese-twin porphyrin coordinating two palladium ions in a square-planar fashion. The absence of any counterions suggested also here an oxidation state of + II for each metal ion.

To obey the square-planar coordination geometry of Pd(II) ions at both coordination sites, the

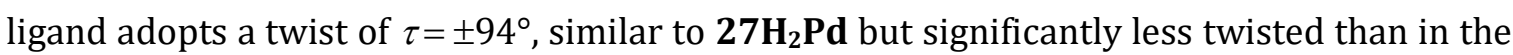
case of $\mathbf{2 7} \mathbf{H}_{2} \mathbf{N i}$ and especially $\mathbf{2 7} \mathbf{N i}_{2}$ (Table 9). Additionally, the incorporation of the second ion bulges the long sides of the ligand backbone leading so to a compression in the length of

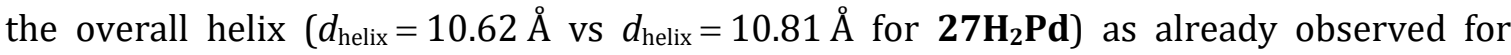
$\mathbf{2 7} \mathbf{H}_{2} \mathbf{N i} / \mathbf{2 7 N i}_{2}{ }^{\left[{ }^{[82}\right]}$ Consequently, the geometric parameters of the $\mathrm{Pd}(\mathrm{II})$ coordination pocket match an almost perfect square planar arrangement with an average angle $\Varangle^{\mathrm{av}}(\mathrm{NMN})=91^{\circ}$ and a deviation of the metal ion center from the $\left\{\mathrm{N}_{4}\right\}$ plane of $d\left(\mathrm{Pd} \cdots\left\{\mathrm{N}_{4}\right\}\right)=0.03 \AA$. 
Unfortunately, no bulk amount of (crystalline) material for $\mathbf{2 7} \mathbf{P d}_{\mathbf{2}}$ could be collected. Most

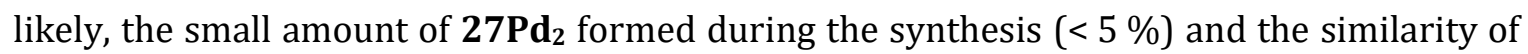
properties between $\mathbf{2 7} \mathbf{H}_{2} \mathbf{P d}$ and $\mathbf{2 7} \mathbf{P d} \mathbf{d}_{2}$ prevent any detection and separation of $\mathbf{2 7} \mathbf{P d}_{2}$ prior

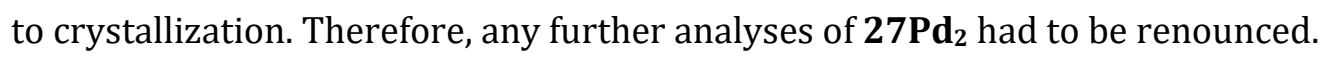

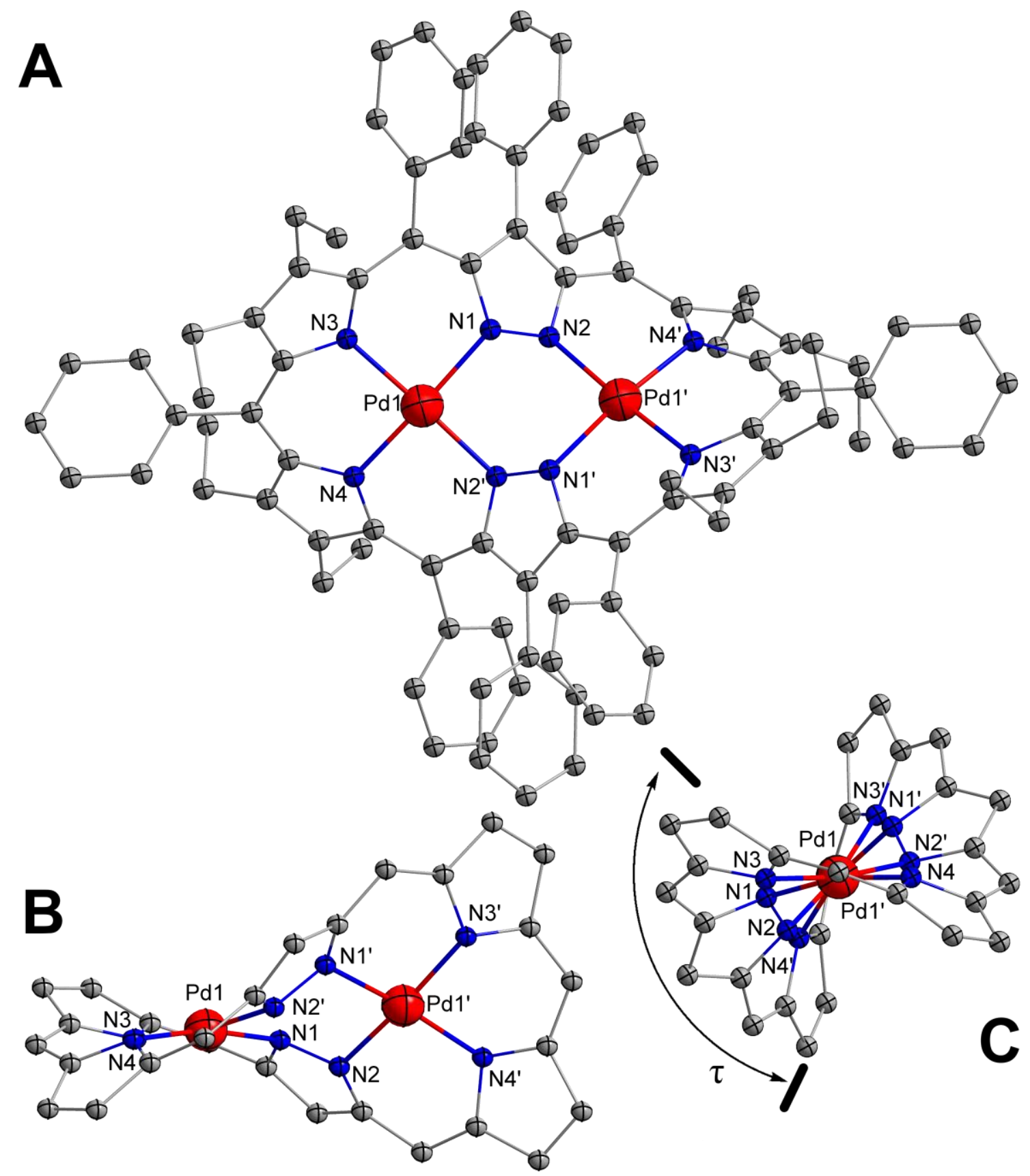

Figure 44: Ball-and-stick representation of the solid state structure of the dipalladium complex of the Siamesetwin porphyrin $\mathbf{2 7 P d _ { 2 }}$ (palladium in red, carbon in grey and nitrogen in blue). Only one enantiomer shown; solvent molecules and hydrogen atoms omitted for clarity. A) Overview including peripheral substituents; B) representation of almost perfectly square planar coordination of the palladium ion and the perpendicular arrangement of the coordination sites with respect to each other, and C) view along the long axis depicting the torsion angle $\tau$. Symmetry transformation for equivalent atoms: $1-x, y, 1 / 2-z$. 


\subsubsection{NMR Spectroscopic Characterization}

Due to a low-spin $\mathrm{d}^{8}$-configuration of $\mathrm{Pd}(\mathrm{II})$, diamagnetic complex $\mathbf{2 7} \mathbf{H}_{2} \mathbf{P d}$ could be

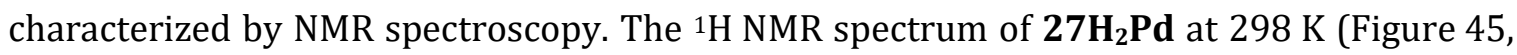
top) shows a $C_{2}$-symmetric molecule with four distinct signals with $\delta<1 \mathrm{ppm}$ that can be assigned to the methyl groups. No resonances of the NH-groups, for the Siamese-twin porphyrin typically found in the deshielded region $(\delta>8 \mathrm{ppm})$, could be detected. Upon cooling to $238 \mathrm{~K}$ (Figure 45 , top inset) one very broad signal at $\delta=9.77 \mathrm{ppm}$ arose. The presence of a single $\mathrm{NH}$-proton peak is in agreement with an overall $C_{2}$-symmetry axis for the macrocycle located along the long axis of the molecule. The proton shift induced by the coordination of the palladium ion observed in solid state is thus retained in solution. However, the broadness of the $\mathrm{NH}$ resonance even at low temperatures suggests fast intramolecular exchange of both protons among the four basic nitrogen atoms of the coordination pocket.

The moderate low-field shift of the pyrrole-NH resonance in comparison to the free-base Siamese-twin porphyrin $\mathbf{2 7 \mathbf { H } _ { 4 }}(\delta=11.37 \mathrm{ppm})$ reflects the increased shielding of the protons and hence a lowered acidity due to the altered protonation pattern in $\mathbf{2 7} \mathbf{H}_{2} \mathbf{P d}$. This observation is in good agreement with the results from the titration experiments of $\mathbf{2 7} \mathbf{H}_{2} \mathbf{P d}$ with DBU (Chapter 3.4.2), where no deprotonation could be achieved.

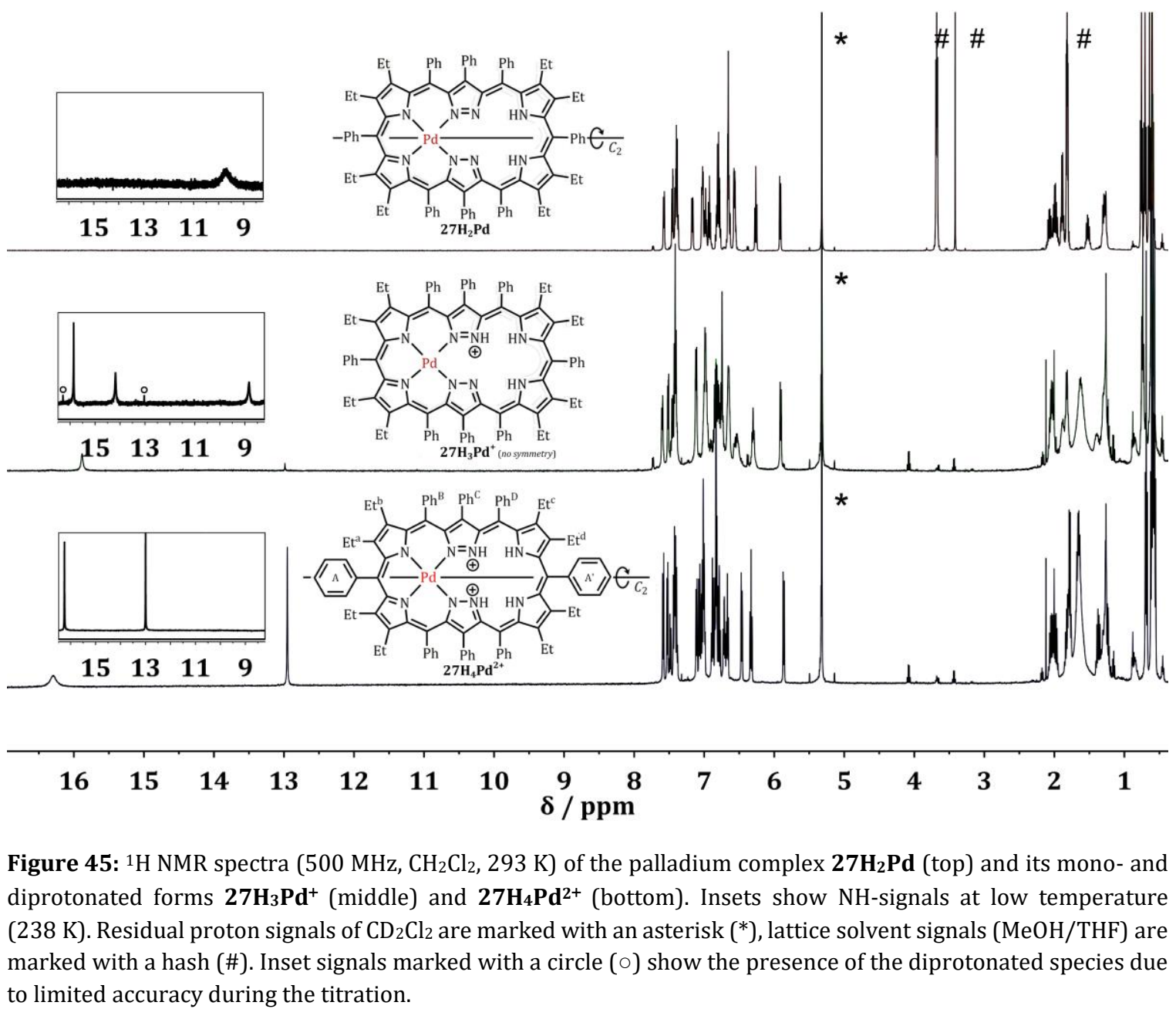


By means of 2D NMR techniques, discrete spin systems could be identified. However, extreme broadening of the NH-group signal even at low temperatures eliminated the possibility to apply any ${ }^{1} \mathrm{H}^{15} \mathrm{~N}$-related 2D NMR techniques and thus exact assignment of peripheral substituents to either coordination site.

The acid/base titration initially followed by UV/vis spectroscopy (Chapter 3.4.2) was additionally examined by NMR and put into comparison with spectra of the neutral monopalladium complex $\mathbf{2 7} \mathbf{H}_{2} \mathbf{P d}$ (Figure 45). The advantage of TFA aside from its high acidity $\left(\mathrm{p} K_{\mathrm{a}}=0.52 \text { in } \mathrm{H}_{2} \mathrm{O}\right)^{[137]}$ is the good solubility in $\mathrm{CH}_{2} \mathrm{Cl}_{2}$ and the absence of any residual protons in the deprotonated trifluoroacetate, which might interfere with compound signals in the ${ }^{1} \mathrm{H}$ NMR spectrum.

At room temperature, all signals of $\mathbf{2} \mathbf{7 H}_{2} \mathbf{P d}$ broaden upon addition of the first equivalent of TFA. The initial two-fold symmetry, previously revealed by four distinct resonances of the methyl groups, is lifted. Only very broad signals can be detected in the deshielded region above $\delta=8.00 \mathrm{ppm}$. The addition of the second equivalent of TFA leads to a resharpening of the entire spectrum and recovery of a peak pattern characteristic for a $C_{2}$-symmetric Siamesetwin porphyrin species: four distinct, although overlapping, resonances associated with the methyl groups. Remarkably, two reasonably sharp signals (NH-proton resonances) are detected already at room temperature in the deshielded region above $\delta=8.00 \mathrm{ppm}$. These changes are consistent with a sequential mono- and diprotonation of the vacant coordination pocket in monopalladium complex $\mathbf{2 7} \mathbf{H}_{2} \mathbf{P d}$ (cf. molecular symmetry in Figure 45). Against this background, each individual spectrum of $\mathbf{2 7} \mathbf{H}_{3} \mathbf{P d}^{+}$and $\mathbf{2 7} \mathbf{H}_{4} \mathbf{P d}^{2+}$ will be analyzed in more detail below.

The spectrum of $\mathbf{2 7} \mathbf{H}_{3} \mathbf{P d}^{+}$does not sharpen significantly upon gradual cooling down to $238 \mathrm{~K}$ (Figure S14). The broad and partially overlapping resonances in the aliphatic and aromatic regions of the spectrum do not allow a reliable assignment of the peaks. However, the resonances of the NH-groups at $238 \mathrm{~K}$ (Figure 45, middle inset) provide some evidence on the nature of $\mathbf{2} \mathbf{7} \mathbf{H}_{3} \mathbf{P d}$.

In the deshielded region $(\delta>8 \mathrm{ppm})$ the spectrum of $\mathbf{2} \mathbf{7 H}_{3} \mathbf{P d}^{+}$shows three peaks $(\delta=15.86$, 14.18 and $8.83 \mathrm{ppm}$ at $238 \mathrm{~K}$ ), with increasing broadness even at lower temperatures and each integral corresponding to one proton. And yet, the resonance of the pyrazole-bound proton at all times appears significantly sharper than signals of pyrrole-bound protons. While fast intramolecular proton exchange affects all protons equally, another effect becomes apparent here: coordination of the pyrazole by the palladium ion in one coordination pocket limits molecular movement of its surrounding and leads to sharpening of associated signals. Two very small signals ( $\delta=16.25$ and $13.00 \mathrm{ppm}$ at $238 \mathrm{~K}$ ) show the presence of the diprotonated species $\mathbf{2 7} \mathbf{H}_{4} \mathbf{P d}^{2+}$, which amounts to around $2.5 \%$ according to integration. This is attributed to the limited accuracy during the titration rather than an equilibrium mixture of species. The asymmetry of $\mathbf{2 7} \mathbf{H}_{3} \mathbf{P d}^{+}$is further supported by the multitude of signals in the ${ }^{13} \mathrm{C}$ NMR spectrum.

Spectra of $\mathbf{2 7} \mathbf{H}_{4} \mathbf{P d}^{2+}$ show already at room temperature sharp signals and a good resolution of the multiplets allowing a full assignment of all peaks. Total protonation additionally increases the rigidity of the overall macrocycle and restricts molecular movement due to 


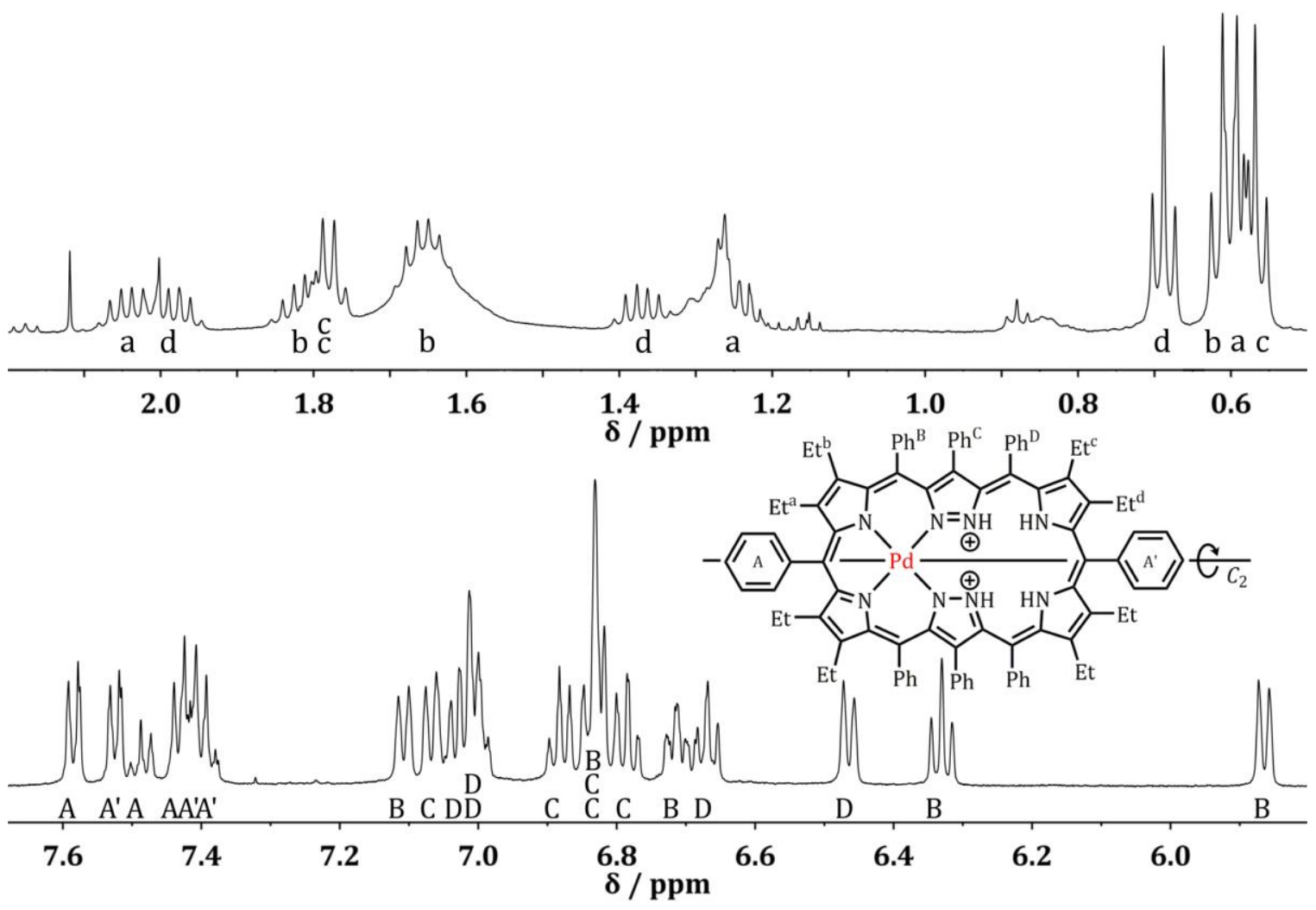

Figure 46: Aliphatic (top) and aromatic (bottom) regions of the ${ }^{1} \mathrm{H}$ NMR spectrum $\left(500 \mathrm{MHz}, \mathrm{CH}_{2} \mathrm{Cl}_{2}, 298 \mathrm{~K}\right)$ of $\mathbf{2 7} \mathbf{H}_{4} \mathbf{P d}^{2+}$. Correlating spin systems are indicated by letters according to inset figure of $\mathbf{2 7} \mathbf{H}_{\mathbf{4}} \mathbf{P d}^{2+}$.

electrostatic repulsion. Letters are used to indicate correlated spin systems according to 1D and 2D NMR spectra (see also Figure S15 and S16).

The aliphatic region (Figure 46, top) shows four distinct spin systems with four triplets $(\delta=0.64-0.50 \mathrm{ppm}$ with $3 \mathrm{~J} \approx 7 \mathrm{~Hz})$ and eight doublets of quartets $(\delta=2.03-1.12 \mathrm{ppm}$ with $3 J \approx 7 \mathrm{~Hz}$ and ${ }^{2} J \approx 14 \mathrm{~Hz}$ ). This pattern is consistent with the presence of eight ethyl groups in a $C_{2}$-symmetric molecule (Figure 46 , inset). Due to the helicity of the macrocycle all methylene protons are diastereotopic and show a geminal coupling. All signals are marginally downfieldshifted in comparison to the free-base ligand $\mathbf{2 7} \mathbf{H}_{4}$ (and $\mathbf{2 7} \mathbf{H}_{2} \mathbf{P d}$ ), reflecting the rather small effect of core variations onto the periphery of the macrocycle.

In the aromatic region (Figure 46, bottom) five distinct spin systems can be found. The signals at $\delta=7.09-5.82 \mathrm{ppm}$ show a characteristic pattern associated with phenyl groups with each spin system containing two doublets (ortho-H) and three triplets (meta- and para-H). These signals are consistent with the three phenyl groups B, C and D on the long side of the macrocycle (Figure 46 , inset). An isolated pattern $(\delta=7.56-7.33 \mathrm{ppm})$ with two distinct spin systems, each containing two doublets and one triplet, represents both phenyl groups (A and $A^{\prime}$ ) located along the symmetry axis of the molecule.

The signals at $\delta=16.25$ and $13.00 \mathrm{ppm}\left(\mathrm{N}^{\mathrm{pz}} \mathrm{H}\right.$ and $\left.\mathrm{Npy}\right)$ are significantly downfield-shifted $(\Delta \delta>2.5 \mathrm{ppm})$ in comparison to the free-base Siamese-twin porphyrin $\mathbf{2 7 \mathbf { H } _ { 4 }}$ as well as the neutral monopalladium complex $\mathbf{2 7} \mathbf{H}_{2} \mathbf{P d}$. These findings are coherent with the view of a 
protonated and therefore strongly deshielding coordination pocket in the monopalladium complex $27 \mathbf{H}_{4} \mathbf{P d}^{2+}$.

${ }^{13} \mathrm{C}$ NMR spectroscopy (Figure S17) of $\mathbf{2} \mathbf{7 H}_{4} \mathbf{P d}^{2+}$ confirmed once again the two-fold symmetry along the long axis of the molecule suggested by the ${ }^{1} \mathrm{H}$ NMR data. With the help of ${ }^{1} \mathrm{H}^{13} \mathrm{C}$ HSQC (Figure S18) and (relative) integration, 49 unique carbon atoms could be identified: $4 \times \mathrm{CH}_{3}, 4 \times \mathrm{CH}_{2}, 21 \times \mathrm{CH}, 16 \times \mathrm{C}_{\mathrm{q}}$ and $4 \times \mathrm{C}_{\mathrm{q}}$ axis. Considering the molecular formula $\mathrm{C}_{92} \mathrm{H}_{84} \mathrm{~N}_{8} \mathrm{Pd}$ as determined by HR-MS and six carbon atoms along the symmetry axis of the macrocycle (Figure 46, inset), this observation is consistent with all previous analyses.

Spatial proximity of correlating spin systems was determined by means of NOESY spectroscopy. Aside from obvious vicinities of ethyl groups of each pyrrole subunit (Eta and $\mathrm{Et}^{\mathrm{b}} / \mathrm{Etc}$ and $\mathrm{Et}$ in Figure S19) and phenyl groups on the long side of the molecule $\left(\mathrm{Ph}^{\mathrm{B}}, \mathrm{Ph}^{\mathrm{C}}\right.$, and $\mathrm{Ph}^{\mathrm{D}}$ in Figure S20), cross-correlations helped to elucidate the total assignment of peripheral substituents with respect to both inequivalent coordination pockets. A selected example of such cross-correlations is presented in Figure 47.

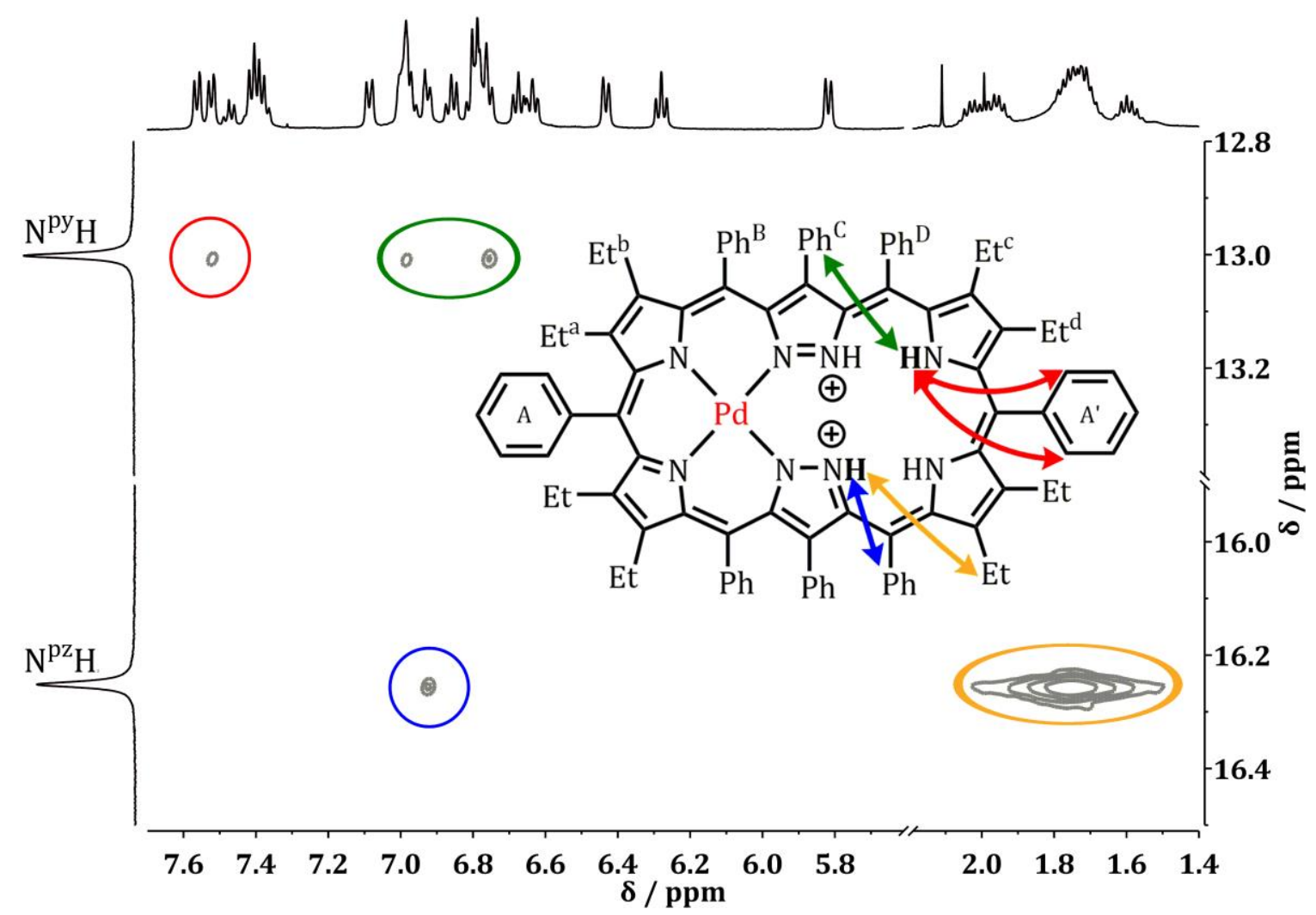

Figure 47: Section ( $\delta=7.6-1.4$ and $16.5-12.8 \mathrm{ppm}$ ) of the NOESY spectrum ( $\left.500 \mathrm{MHz}, \mathrm{CH}_{2} \mathrm{Cl}_{2}, 238 \mathrm{~K}\right)$ of complex $\mathbf{2 7 H}_{4} \mathbf{P d}^{2+}$. Axes were cut where appropriate for better visualization. Relevant spatial proximities are indicated with arrows and their related cross peaks are encircled in corresponding colors.

Peripheral substituents located at the backbone of the metal-free half of the molecule were identified by their interactions with the inner pyrazole and pyrrole protons (Figure 47). In this context it also becomes clear, that the structural features observed in the solid state are fully retained in solution. Thus, the pyrrole-bound protons $(\mathrm{NpyH})$ show cross peaks with ortho-H of $\mathrm{Ph}^{\mathrm{A}^{\prime}}$ (red), while no interaction with phenyl group $\mathrm{Ph}^{\mathrm{D}}$ is evident. Instead, pyrazolebound $\mathrm{Ph}^{\mathrm{C}}$ (green) shows a significant contribution with two NOESY cross peaks. Such 
constellation of interactions can only occur within a twisted structure as in $\mathbf{2 7} \mathbf{H}_{\mathbf{4}} \mathbf{P d} \mathbf{2}^{\mathbf{2}}$ where the up-and-down orientation of the pyrazoles and pyrroles promotes a spatial proximity among initially distant moieties. Further cross peaks between the pyrazole-bound protons $\left(\mathrm{N}^{\mathrm{p}} \mathrm{H}\right)$ and ethyl group Etc (yellow) complete the overall assignment.

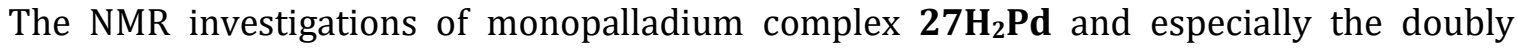
protonated $\mathbf{2 7} \mathbf{H}_{4} \mathbf{P d}^{2+}$ demonstrate clearly the rigidifying effect of the metalation on the inherently flexible expanded porphyrin $\mathbf{2 7 H _ { 4 }}$. 1D and 2D NMR spectroscopy techniques did not only serve to confirm the connectivity of the scaffold but contributed greatly to the illustration of topology of the macrocycle in solution.

\subsubsection{Redox Studies}

Among the metal complexes of the Siamese-twin porphyrin $\mathbf{2 7} \mathbf{H}_{\mathbf{4}}$, monopalladium $\mathbf{2 7} \mathbf{H}_{\mathbf{2}} \mathbf{P d}$ complex offers a unique opportunity to study the redox behavior of a system with one vacant coordination pocket. Complex $\mathbf{2 7} \mathbf{H}_{2} \mathbf{P d}$ distinguishes itself from the previously reported mononickel complex $\mathbf{2 7} \mathbf{H}_{2} \mathbf{N i}$ by superior stability demonstrated e.g. during acid/base titration experiments. Thus, extensive redox studies have been conducted starting with cyclic voltammetry analyses. Both oxidation and reduction events were examined and detailed investigations into the nature of the redox products were performed. Those findings will be presented subsequently according to the applied methods: 1. cyclic voltammetry, 2 . spectroelectrochemistry, 3. electron paramagnetic resonance (EPR) spectroscopy, and 4. complementary density functional theory (DFT) calculations.

\section{Cyclic Voltammetry}

Cyclic voltammetry studies (Figure 48 ) of $\mathbf{2 7}_{2} \mathbf{P d}$ were performed in $\mathrm{CH}_{2} \mathrm{Cl}_{2}$ with $\mathrm{Bu}_{4} \mathrm{NPF}_{6}$ $(0.1 \mathrm{M})$ as the conducting salt. Decamethylferrocene $\left(\mathrm{FeCp}_{2}{ }_{2}\right)$ was added as an internal standard to avoid interference with compound signals. All redox potentials are reported with reference to the ferrocene/ferrocenium redox couple $\left(\mathrm{Fc} / \mathrm{Fc}^{+}\right)$with $E^{0}\left(\mathrm{FeCp}_{2}\right)=-590 \mathrm{mV}$ vs $\mathrm{Fc} / \mathrm{Fc}^{+}$. All spectra were recorded at ambient temperature and under inert conditions.

The reductive region of the cyclic voltammogram (Figure 48A) shows three cathodic waves. The first wave $\left(E_{1 / 2}=-1.59 \mathrm{~V}\right.$ vs $\left.\mathrm{Fc} / \mathrm{Fc}^{+}\right)$is notably separated from the remaining signals and fulfills the criteria for a reversible process when measured separately (Figure 48C). Otherwise the spectrum shows a pattern consistent with two consecutive electron transfers followed by a chemical reaction (EEC-type mechanism): after the initial reduction the current rises dramatically and shows further poorly resolved reduction waves. The reverse scan shows only little to no signals indicating the absence of the previously generated (singly-)reduced species. Considering the structure of $\mathbf{2 7} \mathbf{H}_{2} \mathbf{P d}$, it stands to reason that the first reduction generates reversibly a stable species $\mathbf{2 7} \mathbf{H}_{2} \mathbf{P} \mathbf{d}^{-}$that in turn irreversibly forms and releases $\mathrm{H}_{2}$ from the metal-free coordination pocket upon further reduction. This hypothesis is further supported by the formation of bubbles at the working electrode during the UV/vis spectroscopic monitoring of the described redox processes (see spectroelectrochemical analyses). 

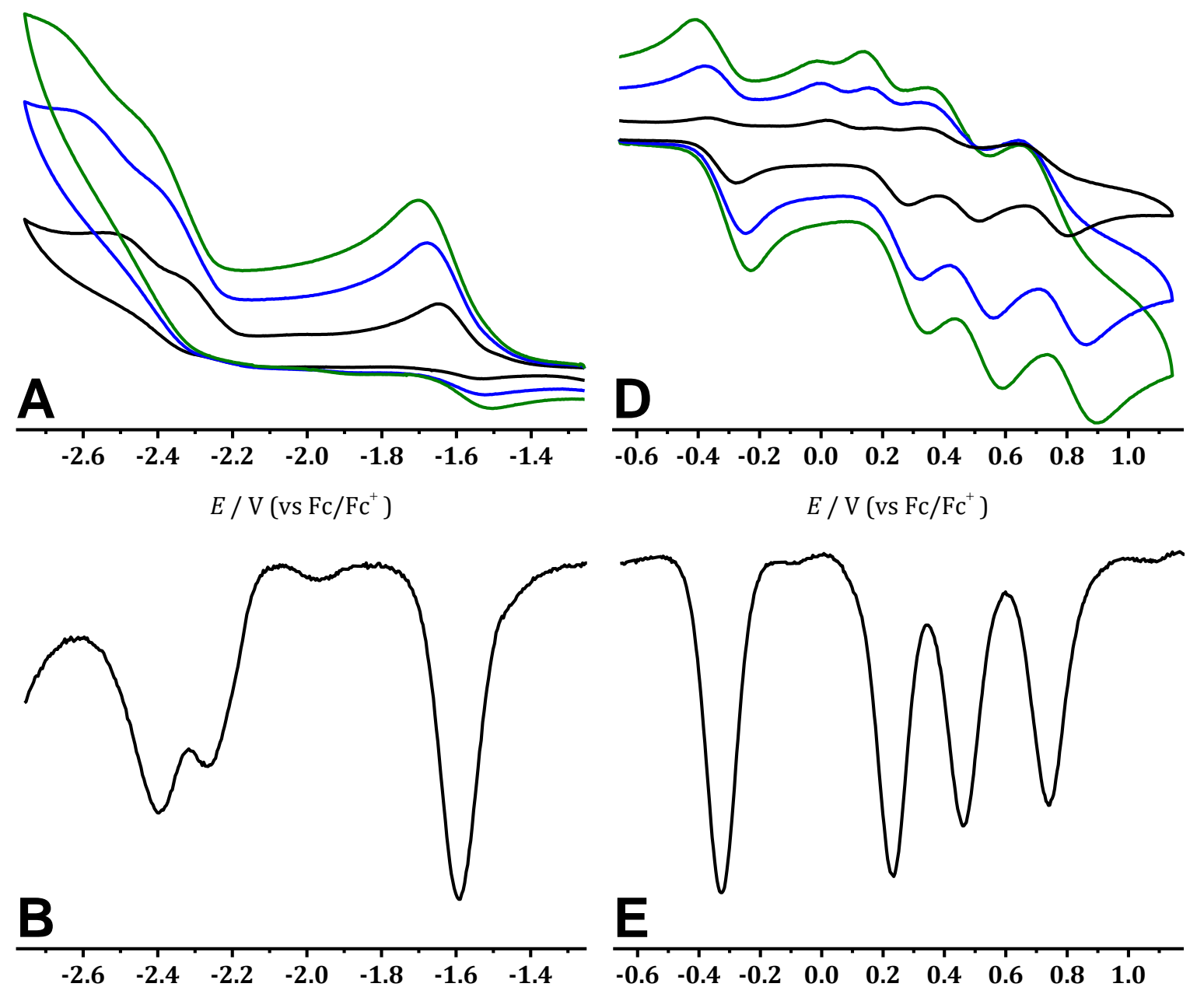

$E / \mathrm{V}\left(\mathrm{vs} \mathrm{Fc} / \mathrm{Fc}^{+}\right)$
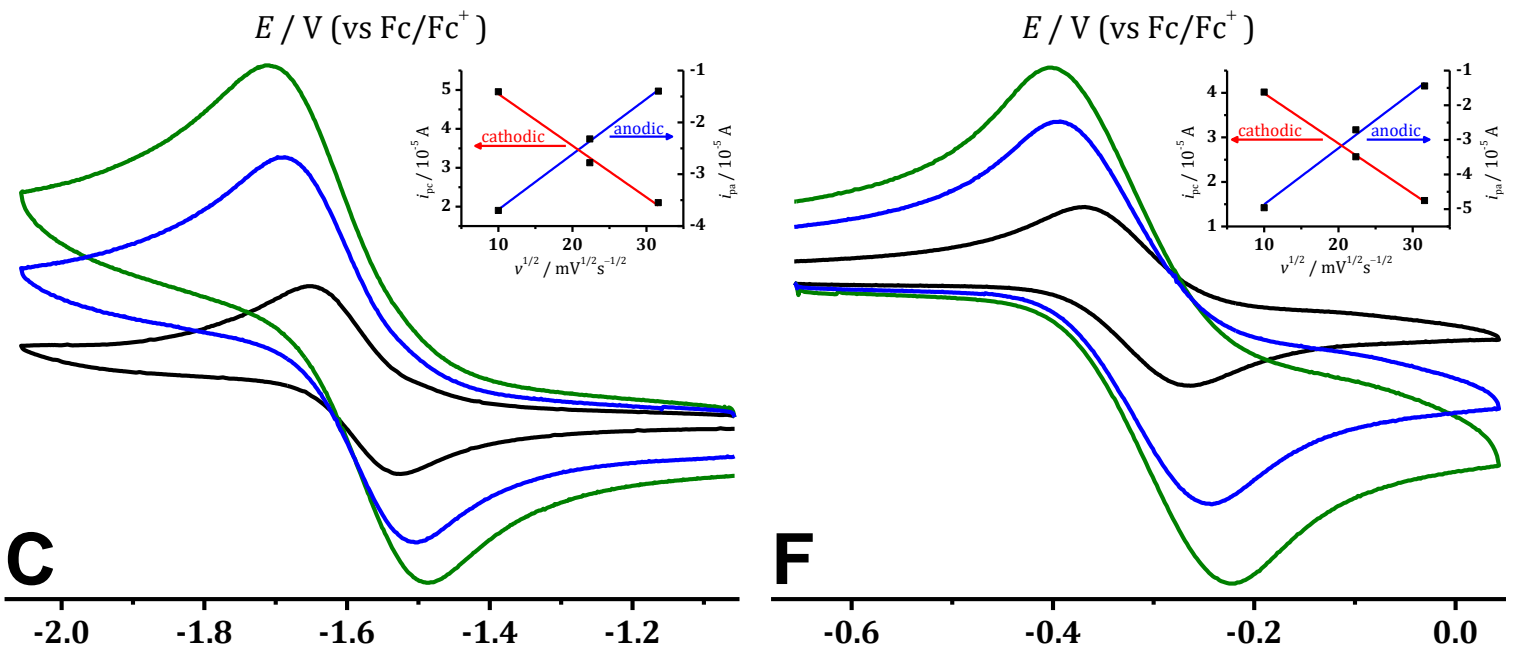

$E / \mathrm{V}\left(\mathrm{vs} \mathrm{Fc} / \mathrm{Fc}^{+}\right)$

$E / \mathrm{V}\left(\mathrm{vs} \mathrm{Fc} / \mathrm{Fc}^{+}\right)$

Figure 48: Cyclic voltammograms (vs Fc/ $\left.\mathrm{Fc}^{+}\right)$of $\mathbf{2 7} \mathbf{H}_{2} \mathbf{P d}\left(\mathrm{CH}_{2} \mathrm{Cl}_{2}, 0.1 \mathrm{M} \mathrm{Bu} 4 \mathrm{NPF}_{6}\right.$, r.t.) at scan rates: $100 \mathrm{mVs}^{-1}$ (black), $500 \mathrm{mVs}^{-1}$ (blue), $1000 \mathrm{mVs}^{-1}$ (green); A) EEC-type reaction, B) complementary square wave voltammogram, $\mathrm{C})$ fully reversible first reduction wave $\left(E_{1 / 2}=-1.59 \mathrm{~V} \mathrm{vs} \mathrm{Fc} / \mathrm{Fc}^{+}\right)$, D) first fully reversible and three quasi-reversible overlapping oxidation waves, E) complementary square wave voltammogram, and F) fully reversible first oxidation wave $\left(E_{1 / 2}=-0.32 \mathrm{~V}\right.$ vs $\left.\mathrm{Fc} / \mathrm{Fc}^{+}\right)$. Half-wave potentials $\left(E_{1 / 2}\right)$ are summarized in Table 10. 
The oxidative region of the cyclic voltammogram (Figure 48D) shows four anodic waves. The first wave $\left(E_{1 / 2}=-0.32 \mathrm{~V}\right.$ vs Fc/Fc $\left./ \mathrm{Fc}^{+}\right)$is notably separated from the remaining signals and fulfills the criteria for a reversible process (Figure $48 \mathrm{~F})$. The subsequent waves $\left(E_{1 / 2}=+0.16,+0.44\right.$ and $+0.74 \mathrm{~V}$ vs $\mathrm{Fc} / \mathrm{Fc}^{+}$) show significant overlapping and are considered quasi-reversible. Upon scanning to even higher potentials (up to $U=+1.5 \mathrm{~V}$ vs $\mathrm{Fc} / \mathrm{Fc}^{+}$) the spectrum worsens increasingly indicating electrochemical decomposition of the analyte. Square wave voltammetry shows by analysis of the corresponding peak areas that all processes are oneelectron transfers.

In the given system, a correlation between the first oxidation potential and the energy of the HOMO as well as between the reduction potential and the energy of the LUMO may be assumed. Thus, the difference $\Delta E^{\text {electro }}=E_{1 / 2}$ (oxidation) $-E_{1 / 2}($ reduction $)=1.27 \mathrm{~V}$ serves as a good approximation of the HOMO-LUMO energy gap.[64,68] This value agrees well with the estimation made from the lowest-energy band on-set in the UV/vis spectrum of $\mathbf{2 7} \mathbf{H}_{2} \mathbf{P d}$ $\left(\lambda \approx 1010 \mathrm{~nm} ; \Delta E^{\text {spectro }}=1.23 \mathrm{eV}\right)$.

In comparison, the cyclic voltammograms of $\mathbf{2 7} \mathbf{H}_{2} \mathbf{N i}$ and $\mathbf{2 7} \mathbf{H}_{4}$ showed no electrochemically reversible redox processes. ${ }^{[82,84]}$ The incorporation of the higher homolog (Pd) seems to increase the stability of the system and allows so the study of the redox behavior of the monometalated species $\mathbf{2 7} \mathbf{H}_{\mathbf{2}} \mathbf{P d}$. Thus, monopalladium complex $\mathbf{2 7} \mathbf{H}_{\mathbf{2}} \mathbf{P d}$ is the first representative with a vacant coordination pocket among the Siamese-twin porphyrin complexes to be analyzed in detail towards the properties of the singly oxidized $\left(\mathbf{2 7} \mathbf{H}_{2} \mathbf{P d} \mathbf{d}^{+}\right)$ and singly reduced $\left(\mathbf{2 7} \mathbf{H}_{2} \mathbf{P d} \mathbf{d}^{-}\right)$products. Especially the reduction had not yet been investigated in complexes of the Siamese-twin porphyrin $\mathbf{2 7} \mathbf{H}_{4}$.

Table 10: Half-wave potentials* $\left(E_{1 / 2}\right)$ for (quasi-)reversible redox processes of the Siamese-twin porphyrin

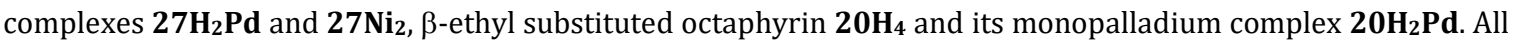
potentials in $\mathrm{V}$ ( $\mathrm{vs} \mathrm{Fc} / \mathrm{Fc}^{+}$).

\begin{tabular}{|c|c|c|c|c|}
\hline & $27 \mathrm{H}_{2} \mathrm{Pd}$ & $27 \mathrm{Ni}_{2}$ & $20 \mathrm{H}_{4}$ & $20 \mathrm{H}_{2} \mathrm{Pd}$ \\
\hline $2^{\text {nd }}$ reduction & - & - & -1.97 & -2.00 \\
\hline $1^{\text {st }}$ reduction & -1.59 & -1.90 & -1.66 & -1.57 \\
\hline $1^{\text {st }}$ oxidation & -0.32 & -0.16 & -0.23 & -0.32 \\
\hline $2^{\text {nd }}$ oxidation & +0.16 & +0.30 & +0.11 & +0.13 \\
\hline $3^{\text {rd }}$ oxidation & +0.44 & +0.66 & +1.12 & +1.07 \\
\hline $4^{\text {th }}$ oxidation & +0.74 & - & - & - \\
\hline
\end{tabular}

${ }^{*} E_{1 / 2}=\left(E_{\mathrm{pc}}+E_{\mathrm{pa}}\right) / 2$; measurements were conducted in $\mathrm{CH}_{2} \mathrm{Cl}_{2}\left(0.1 \mathrm{M} \mathrm{Bu} 4 \mathrm{NPF}_{6}\right.$, r.t. $)$ at a scan rate of $100 \mathrm{mVs}^{-1}$.

Search for suitable comparison examples among expanded porphyrins revealed a remarkable

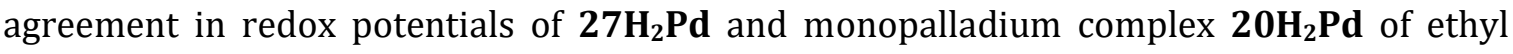
substituted octaphyrin $\mathbf{2 0 H}_{4}$ (Table 10).[64] The figure-eight shaped complex $\mathbf{2 0 H}_{2} \mathbf{P d}$ shows overall five reversible redox steps at $E_{1 / 2}=-2.00,-1.57,-0.32,+0.13$, and $+1.07 \mathrm{~V}$ vs Fc $/ \mathrm{Fc}^{+}$. The processes at $E_{1 / 2}=-1.57,-0.32$, and $+0.13 \mathrm{~V}$, relevant for this comparison, are all considered to be ligand based and transition metal coordination is assumed to facilitate the respective oxidation or reduction reaction with regard to the parent free-base octaphyrin 
$\mathbf{2 0 H}_{4}{ }^{[64]}$ Also the Siamese-twin porphyrin scaffold in complexes $\mathbf{2 7} \mathbf{N i}_{2}$ and $\mathbf{2 7} \mathbf{C u}_{2}$ was shown to act as a non-innocent ligand. ${ }^{[82,83]}$ Although the connectivity of the Siamese-twin porphyrin $\mathbf{2 7 H}_{4}$ with its six meso-linked subunits resembles the shape of hexaphyrins, it seems that its electronic structure and topology is much more closely related to the figure-eight nonaromatic octaphyrins such as $\mathbf{2 0 H _ { 4 }}$.

\section{Spectroelectrochemistry}

Both electrochemically reversible redox processes $\left(E_{1 / 2}=-1.59\right.$ and $\left.+0.32 \mathrm{~V}\right)$ were monitored $\mathrm{UV} /$ vis spectroscopically (Figure 49) to gain further insights into the nature of $\mathbf{2} \mathbf{7} \mathbf{H}_{\mathbf{2}} \mathbf{P} \mathbf{d}^{-}$and $\mathbf{2 7} \mathbf{H}_{2} \mathbf{P d}^{+}$. Spectroelectrochemical investigations were performed using quartz cuvettes with electrochemical conditions adopted from cyclic voltammetry. Monitoring of the spectrochemical reaction required scanning through the platinum net at fast scans rates and thus led to a high noise to signal ratio of the recorded spectra.

Application of a steady reducing potential $\left(U=-1.9 \mathrm{~V}\right.$ vs $\left.\mathrm{Fc} / \mathrm{Fc}^{+}\right)$led to continuous changes in all major features in the UV/vis spectrum of $\mathbf{2} \mathbf{7} \mathbf{H}_{2} \mathbf{P d}$ (Figure 49, right, black trace). Spectra were recorded every $12 \mathrm{~s}$ until no further changes could be detected $\left(\mathbf{2 7} \mathbf{H}_{2} \mathbf{P d} \mathbf{d}^{-}\right.$; red trace). Successive application of a steady re-oxidizing potential $\left(U=-0.7 \mathrm{~V} \mathrm{vs} \mathrm{Fc} / \mathrm{Fc}^{+}\right)$resulted in the stepwise recovery of the initial spectrum of $\mathbf{2 7}_{2} \mathbf{P d}$ (black trace). Fast scanning needed to be applied to ensure full reversibility of the spectral changes. This may partially be attributed to the pseudo-inert spectroelectrochemical cuvette which was charged with anhydrous solvents but operated under ambient conditions, and partially to the redox process at hand. The EECtype process observed during cyclic voltammetry measurements (cf. Figure 48A) also becomes evident during the UV/vis spectroscopic monitoring: over time tiny bubbles are forming at the platinum net, interfering with the measurement (high noise level) and reversibility of the redox process.

While the SORET-like band ( $\lambda=408 \mathrm{~nm}$ ) experiences a bathochromic shift, the Q-like band $(\lambda=662 \mathrm{~nm})$ loses half of its intensity and the shoulder at $\lambda=477 \mathrm{~nm}$ completely depletes. No reliable conclusion can be drawn on the faith of the band at $\lambda=799 \mathrm{~nm}$ due to extensive
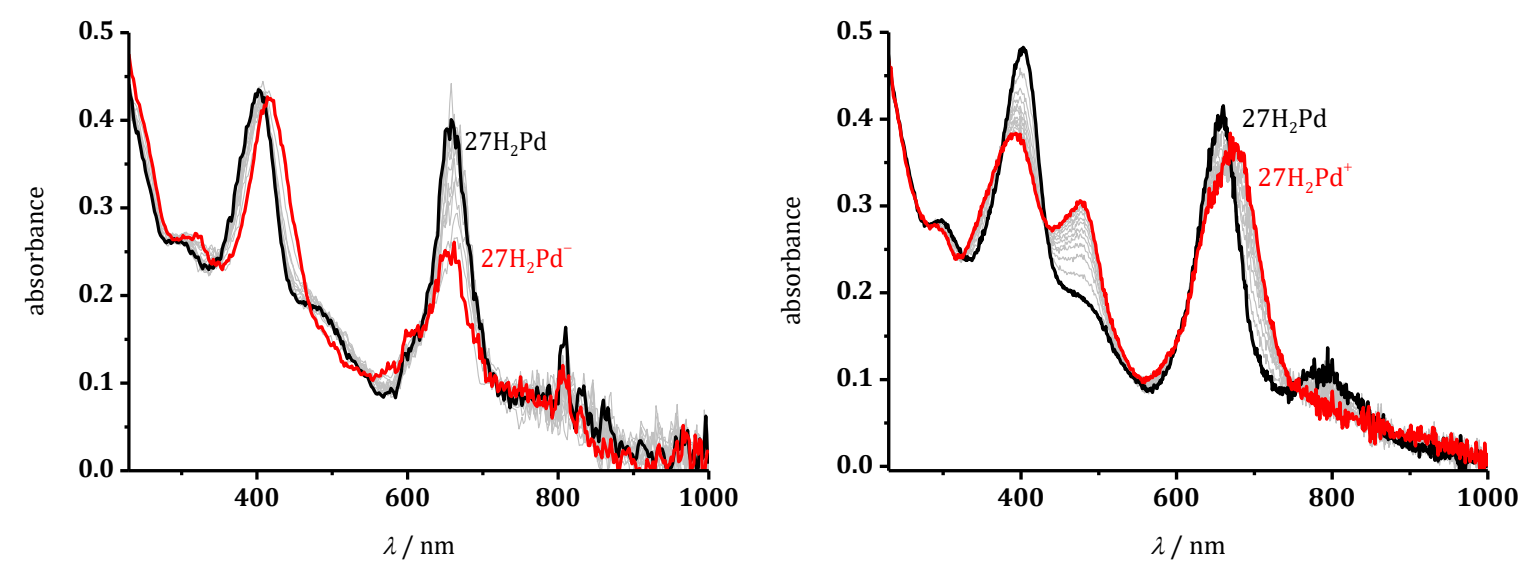

Figure 49: UV/vis spectra of $\mathbf{2 7} \mathbf{H}_{2} \mathbf{P d}$ (black trace) following the electrochemical reduction to give $\mathbf{2 7} \mathbf{H}_{2} \mathbf{P d} \mathbf{d}^{-}$(left; in red, one scan every $12 \mathrm{~s}$ ) and oxidation to give $\mathbf{2 7} \mathbf{H}_{2} \mathbf{P d}^{+}$(right; in red, one scan every $2 \mathrm{~min}$ ) in $\left(\mathrm{CH}_{2} \mathrm{Cl}_{2}, 0.1 \mathrm{M}\right.$ $\mathrm{Bu}_{4} \mathrm{NPF}_{6}$, r.t.). 
noise. Because of the fast evolution of gas as well as the distinctly lower reduction potential compared to previously reported bimetallic complexes, ${ }^{[82]}$ the reduction must be located at the metal-free site of the macrocycle (Figure 50A).

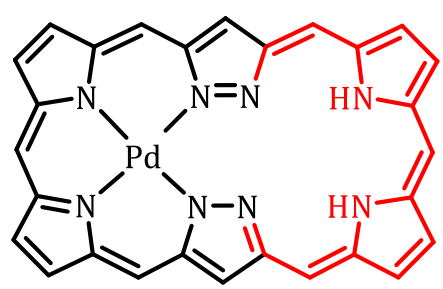

A

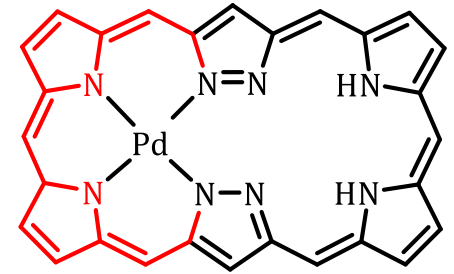

$\mathbf{B}$

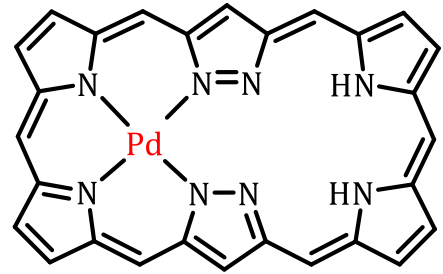

C

Figure 50: Representations of possible loci of redox activity of one electron oxidation or reduction in $27 \mathbf{H}_{2} \mathbf{P d}$ (indicated in red): A) Ligand-based oxidation center located on the metal-free site of the macrocycle, B) ligandbased oxidation center located on the metal-containing site of the macrocycle and C) metal-ion centered oxidation. Peripheral substituents were omitted for clarity.

Application of a steady oxidative potential $\left(U=-0.2 \mathrm{~V}\right.$ vs $\left.\mathrm{Fc} / \mathrm{Fc}^{+}\right)$led to continuous changes in all major features in the UV/vis spectrum of $\mathbf{2} \mathbf{7} \mathbf{H}_{2} \mathbf{P d}$ (Figure 49 , right, black trace). Spectra were recorded every 2 min until no further changes could be detected $\left(\mathbf{2 7}_{\mathbf{H}} \mathbf{P} \mathbf{d}^{+}\right.$; red trace). Successive application of a steady re-reducing potential $\left(U=-0.7 \mathrm{~V} \mathrm{vs} \mathrm{Fc} / \mathrm{Fc}^{+}\right)$resulted in the stepwise recovery of the initial spectrum of $\mathbf{2 7} \mathbf{H}_{2} \mathbf{P d}$ (black trace). The fact that slower scan rates could be applied during the oxidation, points towards a higher stability of $\mathbf{2} \mathbf{7} \mathbf{H}_{2} \mathbf{P d} \mathbf{d}^{+}$with respect to $\mathbf{2 7} \mathbf{H}_{2} \mathbf{P d}^{-}$.

Conversely to the changes upon reduction, the SORET-like band $(\lambda=408 \mathrm{~nm})$ loses significantly in intensity while the palladium-specific, Q-like band $(\lambda=662 \mathrm{~nm})$ experiences a bathochromic shift. Meanwhile, the shoulder at $\lambda=477 \mathrm{~nm}$ grows into a distinctive band. The same shoulder already proved particularly sensitive to protonation (Chapter 3.4.2) thus suggesting that the oxidation process involves the same subunits as those involved in the protonation, namely the central pyrazole moieties. The contrasting development of the $\mathrm{UV} /$ vis features during oxidation of $\mathbf{2 7} \mathbf{H}_{2} \mathbf{P d}$ when compared to the reduction points toward a ligand-based process centered on the metal-containing site of the complex (Figure 50B). This consideration is further supported by electrostatic reasoning: due to the separated conjugation pathways of the macrocycle an oxidation of the doubly deprotonated, metalcontaining ligand site (B) appears more favorable than oxidation of the metal ion (C) or the ligand on the metal-free site $(\mathrm{A})$.

\section{EPR Spectroscopy}

To further investigate the nature of the oxidation and reduction products, bulk electrolysis of a solution of $\mathbf{2 7} \mathbf{H}_{2} \mathbf{P d}$ (same conditions as spectroelectrochemistry set-up, strictly inert conditions) was performed and EPR samples were prepared from these solutions. The progress of the electrolysis was monitored on the basis of the current flow and an estimate on the total charge count $(Q)$. Additionally, a blind sample of non-electrolyzed $\mathbf{2 7} \mathbf{H}_{2} \mathbf{P d}$ was prepared to exclude any EPR-active species prior to electrolysis. 
Electrolysis at a steady reductive potential yielded a green solution of $\mathbf{2 7} \mathbf{H}_{2} \mathbf{P d}^{-}$. The sample was immediately transferred into an EPR tube, frozen in liquid $\mathrm{N}_{2}$ and an EPR spectrum (X-band, Figure 51A) was measured at $153 \mathrm{~K}$. The electrochemical product $\mathbf{2 7} \mathbf{H}_{\mathbf{2}} \mathbf{P d}^{-}$shows an almost isotropic spectrum (Figure 51A) with $g_{1}=g_{2}=2.00$ and $g_{3}=2.01$ (Table 11) as might be expected for an organic radical anion. The signal is narrow $\left(W_{1}=W_{2}=0.0\right.$ and $W_{3}=2.4 \mathrm{mT}$ with Gaussian line shapes), but low in intensity. The low concetration of EPRactive species is in line with the high sensitivity of the set-up towards the applied voltage (onset of EEC-mechanism). The shape and position support the presence of an organic radical with no further interactions with the metal ion. The spectrum can be reproduced with remarkable agreement also by chemical reduction (Figure 51B; reduction with 1.0 eq of $\mathrm{CoCp}_{2}{ }_{2}$ in $\mathrm{CH}_{2} \mathrm{Cl}_{2}$ ).

Electrolysis at a steady oxidative potential yielded a brown solution of $\mathbf{2 7} \mathbf{H}_{2} \mathbf{P} \mathbf{d}^{+}$. The sample was immediately transferred into an EPR tube, frozen in liquid $\mathrm{N}_{2}$ and an EPR spectrum
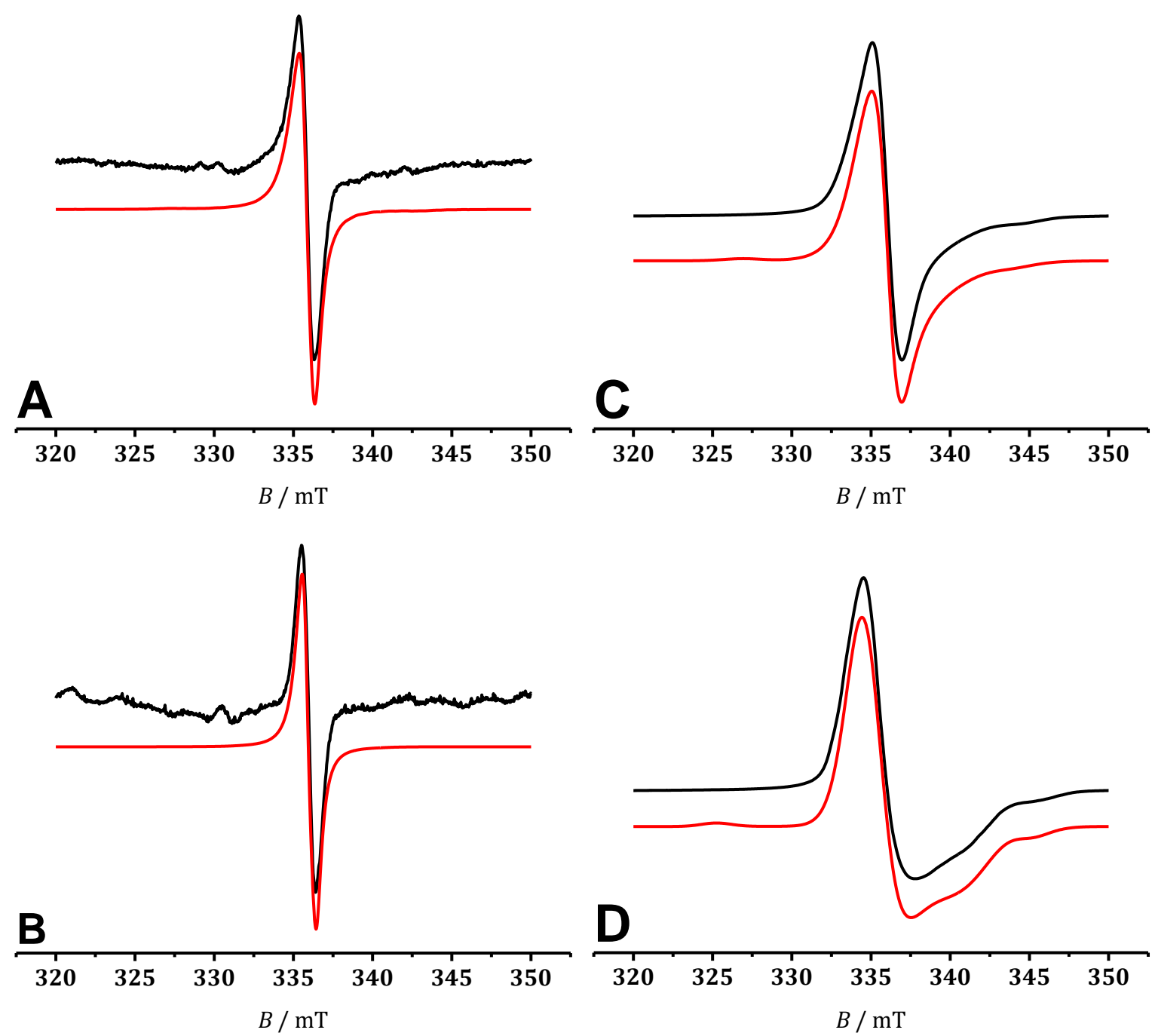

Figure 51: EPR spectra (black traces) and simulations (red traces, Gaussian line shapes) of samples from redox investigations of $\mathbf{2 7} \mathbf{H}_{2} \mathbf{P d}$ : A) electrochemical reduction product $\mathbf{2 7} \mathbf{H}_{2} \mathbf{P d}^{-}\left(\mathrm{CH}_{2} \mathrm{Cl}_{2}, 153 \mathrm{~K}\right)$; B) chemical reduction product $\mathbf{2 7} \mathbf{H}_{2} \mathbf{P d}^{-}\left(\mathrm{CH}_{2} \mathrm{Cl}_{2}, 148 \mathrm{~K}\right)$; C) electrochemical oxidation product $\mathbf{2 7} \mathbf{H}_{2} \mathbf{P d}^{+}\left(\mathrm{CH}_{2} \mathrm{Cl}_{2}, 153 \mathrm{~K}\right)$, and D) chemical oxidation product $\mathbf{2 7} \mathbf{H}_{2} \mathbf{P d}^{+}\left(\mathrm{CH}_{2} \mathrm{Cl}_{2}, 148 \mathrm{~K}\right)$. Corresponding parameters are reported in Table 11.[138,139] 
Table 11: EPR simulation parameters for electrochemically and chemically prepared samples $\mathbf{2 7} \mathbf{H}_{2} \mathbf{P d}^{+}$and 27. $\mathbf{H}_{2} \mathbf{P d}^{-} \cdot[138,139]$

\begin{tabular}{|c|c|c|c|c|}
\hline & 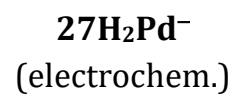 & $\begin{array}{c}\mathbf{2 7 H}_{2} \mathbf{P d}^{-} \\
\text {(chem.) }\end{array}$ & $\begin{array}{c}\mathbf{2 7} \mathbf{H}_{2} \mathbf{P d}^{+} \\
\text {(electrochem.) }\end{array}$ & $\begin{array}{r}27 \mathbf{H}_{2} \mathbf{P d}^{+} \\
\text {(chem.) }\end{array}$ \\
\hline$g_{1}$ & 2.00 & 2.00 & 1.99 & 1.98 \\
\hline$g_{2}$ & 2.01 & 2.01 & 2.00 & 2.01 \\
\hline$g_{3}$ & 2.01 & 2.01 & 2.01 & 2.01 \\
\hline$g_{\mathrm{av}^{\mathrm{a}}}$ & 2.01 & 2.01 & 2.00 & 2.00 \\
\hline$W_{\text {freq }}[\mathrm{mT}]$ & 2.2 & 1.8 & 1.5 & 3.8 \\
\hline$W_{1}[\mathrm{mT}]$ & - & - & 7.2 & 3.9 \\
\hline$W_{2}[\mathrm{mT}]$ & - & - & 1.0 & 3.1 \\
\hline$W_{3}[\mathrm{mT}]$ & 2.4 & 1.5 & 2.5 & 1.1 \\
\hline
\end{tabular}

${ }^{\mathrm{a}} g_{\mathrm{av}}=\left[\left(g_{1}{ }^{2}+g_{2}{ }^{2}+g_{3}{ }^{2}\right) / 3\right]^{1 / 2}$

(X-band, Figure 51B) was measured at $153 \mathrm{~K}$. The electrochemical product $\mathbf{2 7} \mathbf{H}_{2} \mathbf{P d}^{+}$shows an almost isotropic spectrum (Figure 51C) with $g_{1}=1.99, g_{2}=2.00$ and $g_{3}=2.01$ (Table 11) as might be expected for an organic radical cation. However, the broadness of the signal $\left(W_{1}=7.2, W_{2}=1.0\right.$ and $W_{3}=2.5 \mathrm{mT}$ with Gaussian line shapes) suggests also partial spin density distribution and unresolved hyperfine coupling to the palladium ion, as already deduced from the spectroelectrochemical investigation. Due to the poorly featured shape, line broadness and the low contribution of the palladium ${ }^{105} \mathrm{Pd}$ isotope $(I=5 / 2$ with $22.3 \%$ abundance amount to $\sim 4 \%$ of spectral intensity),[140] no reliable simulation for the hyperfine coupling constant $A_{\text {Pd }}$ could be achieved. Considering the connectivity in complex $\mathbf{2 7} \mathbf{H}_{2} \mathbf{P d}$ and reports on EPR investigations of $\mathbf{2 7} \mathbf{C u}_{2},{ }^{[83]}$ further coupling to the coordinating nitrogen atoms (coupling constant $A_{\mathrm{N}}$ ) may as well be concealed by the broadness of the present lines. Nonetheless, the spectrum supports the conclusion that the oxidation of the Siamese-twin porphyrin complex $\mathbf{2 7} \mathbf{H}_{2} \mathbf{P d}$ takes place at the metal-containing porphyrin pocket (Figure 50B). The chemically oxidized sample of $\mathbf{2 7} \mathbf{H}_{2} \mathbf{P d} \mathbf{d}^{+}$(Figure 51D; oxidation in $\mathrm{CH}_{2} \mathrm{Cl}_{2}$ with 1.0 eq of $\mathrm{AgBF}_{4}$ in nitroethane) strongly differs in color (teal-color sample), suggesting thus partial decomposition during bulk electrolysis (brown sample). Otherwise, the EPR spectrum differs only slightly in the broadness of the lines which can be attributed to the different ionic environment in the samples according to preparation $\left(0.1 \mathrm{M} \mathrm{Bu}_{4} \mathrm{NPF}_{6}\right.$ in $\mathrm{CH}_{2} \mathrm{Cl}_{2}$ vs $\mathrm{CH}_{2} \mathrm{Cl}_{2}+\mathrm{AgBF}_{4}$ in nitroethane).

Porphyrin-centered oxidation reactions are well known for, e.g. 26Pd, ${ }^{[15,141]}$ and also the Siamese-twin porphyrin $\mathbf{2 7} \mathbf{H}_{4}$ showed the redox non-innocence of its macrocyclic backbone in bimetallic complexes (27- $\mathbf{C u}_{2}$ and $\left.\mathbf{2 7} \mathbf{N i}_{2}\right)$. ${ }^{[82,83]}$ Beyond the established participation of the ligand framework in redox reactions, monometalated complex $\mathbf{2 7}_{\mathbf{2}} \mathbf{P d}$ reveals the split personality of the electronic structure in the Siamese-twin porphyrin: the reduction occurs at the metal-free hemicycle and the oxidation takes place at the metal-containing half of the molecule. 


\section{DFT Calculations}

Evaluation of the redox chemistry was further complemented by computational methods. Thereto geometry optimizations on the basis of coordinates from the X-ray molecular structure of $\mathbf{2} \mathbf{7} \mathbf{H}_{2} \mathbf{P d}$ were performed. Due to the size of the system, cost-efficient basis sets and functionals were applied first (cf. Chapter 6.3 for details). Subsequently, the optimized coordinates were used for single point energy calculations and orbital analyses on a more elaborate computational level. Though this approach might be considered less accurate, the good agreement with spectroscopic results justifies the expeditious method. Furthermore, only slight changes in the molecular conformation were detected upon relaxation of the molecular structures (Figure S22 and S23). Firstly, the size of the HOMO-LUMO gap and the location of the frontier orbitals was estimated from the final total energies (Figure 52). Additionally, spin density distributions for $\mathbf{2 7} \mathbf{H}_{2} \mathbf{P d} \mathbf{d}^{+}$and $\mathbf{2 7} \mathbf{H}_{2} \mathbf{P d}^{-}$were calculated (Figure 53). Herein, coordinates from the $\mathrm{X}$-ray molecular structure of $\mathbf{2 7} \mathbf{H}_{\mathbf{2}} \mathbf{P d}$ were used and geometry optimizations were waived due to cost-benefit consideration.

The HOMO-LUMO gap of $\mathbf{2 7}_{\mathbf{2}} \mathbf{P d}$ with a cis-arrangement of the NH-protons in the metal-free coordination pocket amounts to $\Delta E^{\text {calc }}=1.35 \mathrm{eV}$. This value is in good agreement with previous estimations from cyclic voltammetry $\left(\Delta E^{\text {electro }}=1.27 \mathrm{~V}\right)$ and $\mathrm{UV} / \mathrm{vis}$ spectroscopy $\left(\Delta E^{\text {spectro }}=1.23 \mathrm{eV}\right)$. A hypothetical trans-arrangement of the NH-protons, as observed in the parent free-base ligand $\mathbf{2 7} \mathbf{H}_{4}$, was probed as well and led to a significant increase in the HOMO-LUMO gap separation to $\Delta E^{\text {calc }}=1.84 \mathrm{eV}$, thus supporting further the correct assignment of the protonation pattern in a cis-manner from X-ray crystallographic data and NMR spectroscopic analyses. Further, comparison of the final single point energies shows a higher stabilization of the cis-tautomer by $\Delta E^{\text {stabilization }}=1.25 \mathrm{kcal} / \mathrm{mol}$. The palladium-induced tautomerism in the vacant $\left\{\mathrm{N}_{4}\right\}$-coordination pocket does not only decrease the HOMO-LUMO gap, but has additionally an enormous effect on the shape and location of the frontier orbitals
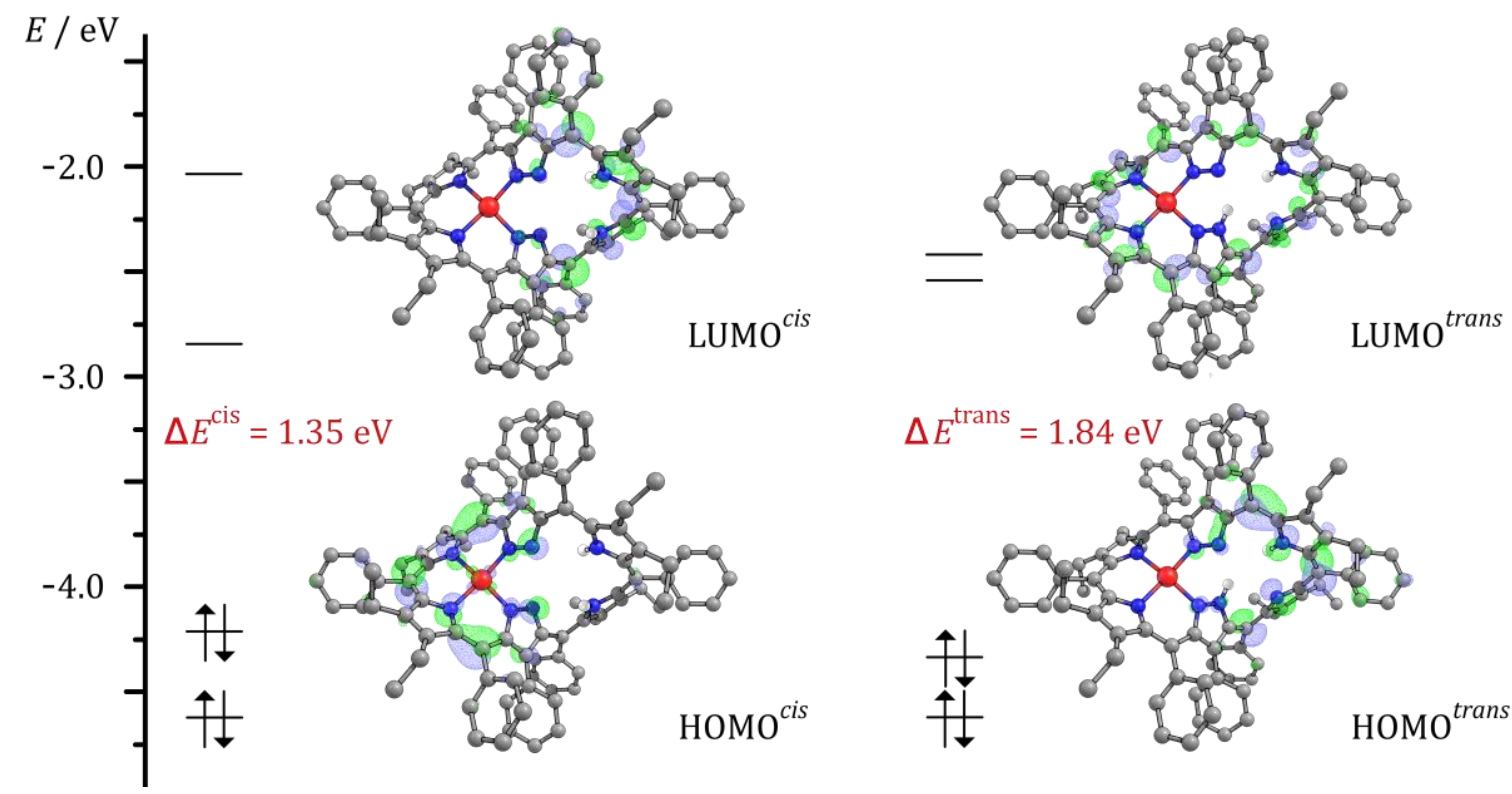

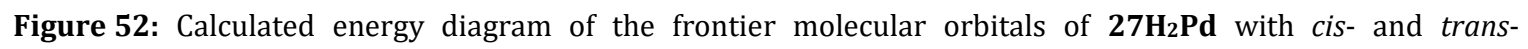
arrangement of the $\mathrm{NH}$-protons and complimentary contour plots (blue: positive, green: negative; contour value: $0.04)$ of the HOMO $(\alpha / \beta\langle 353\rangle)$ and LUMO $(\alpha / \beta\langle 354\rangle)$. Carbon-bound hydrogen atoms omitted for clarity. 
(Figure 52) and therefore an immediate impact on the redox behavior of complex $\mathbf{2 7} \mathbf{H}_{2} \mathbf{P d}$. Not only are $\mathrm{HOMO}^{\text {cis }}$ and HOMO trans located on opposite sides of the hemicycles, but also the LUMOs change from a ligand-wide frontier orbital (LUMOtrans) to a hemicircular-shape for LUMO ${ }^{\text {cis }}$ (Figure 52). Consequently, incorporation of the palladium ion in one coordination pocket evokes a rearrangement of the protonation pattern in the second, vacant site and by implication determines the redox behavior of the entire complex $\mathbf{2 7} \mathbf{H}_{2} \mathbf{P d}$.

In agreement with the delocalization of the HOMOcis on the metal-coordinating and the LUMO $^{\text {cis }}$ on the metal-free site of the ligand in $\mathbf{2 7} \mathbf{H}_{\mathbf{2}} \mathbf{P d}$ (Figure 52), calculations and visualizations of the spin density distribution for $\mathbf{2 7} \mathbf{H}_{2} \mathbf{P d} \mathbf{d}^{+}$and $\mathbf{2 7} \mathbf{H}_{2} \mathbf{P d}^{-}$(Figure 53) revealed the split electronic nature of the macrocycle.

Analyses of the involved orbitals showed mainly organic character for both radical species. Singly reduced complex $\mathbf{2 7} \mathbf{H}_{2} \mathbf{P d}^{-}$(Figure 53, left) shows a spin density distribution centered on the metal-free half of the complex with a large fraction delocalized over the carbon atoms along the conjugation pathway. Only minor fractions can be found at the four nitrogen atoms. Such spin density distribution is in good agreement with the previously described almost isotropic EPR-spectrum of $\mathbf{2} \mathbf{7} \mathbf{H}_{2} \mathbf{P d}^{-}$with no observable hyperfine interactions with neighboring nitrogen atoms.

Singly oxidized complex $\mathbf{2 7} \mathbf{H}_{2} \mathbf{P d}^{+}$(Figure 53, right) shows a spin density distribution centered on the palladium-coordinating half of the complex with a minor fraction located at the central metal ion. Delocalization of spin density in $\mathbf{2 7} \mathbf{H}_{2} \mathbf{P} \mathbf{d}^{+}$mainly involves the mesocarbon atoms, the pyrrolic and the imine-like nitrogen atoms of the pyrazole. Also, this image is in good agreement with the previously described almost isotropic EPR-spectrum of $\mathbf{2 7}_{\mathbf{2}} \mathbf{P d}^{+}$. Unresolved hyperfine interactions of the unpaired electron with the palladium ion and nitrogen atoms may lead to a broad and featureless spectrum.
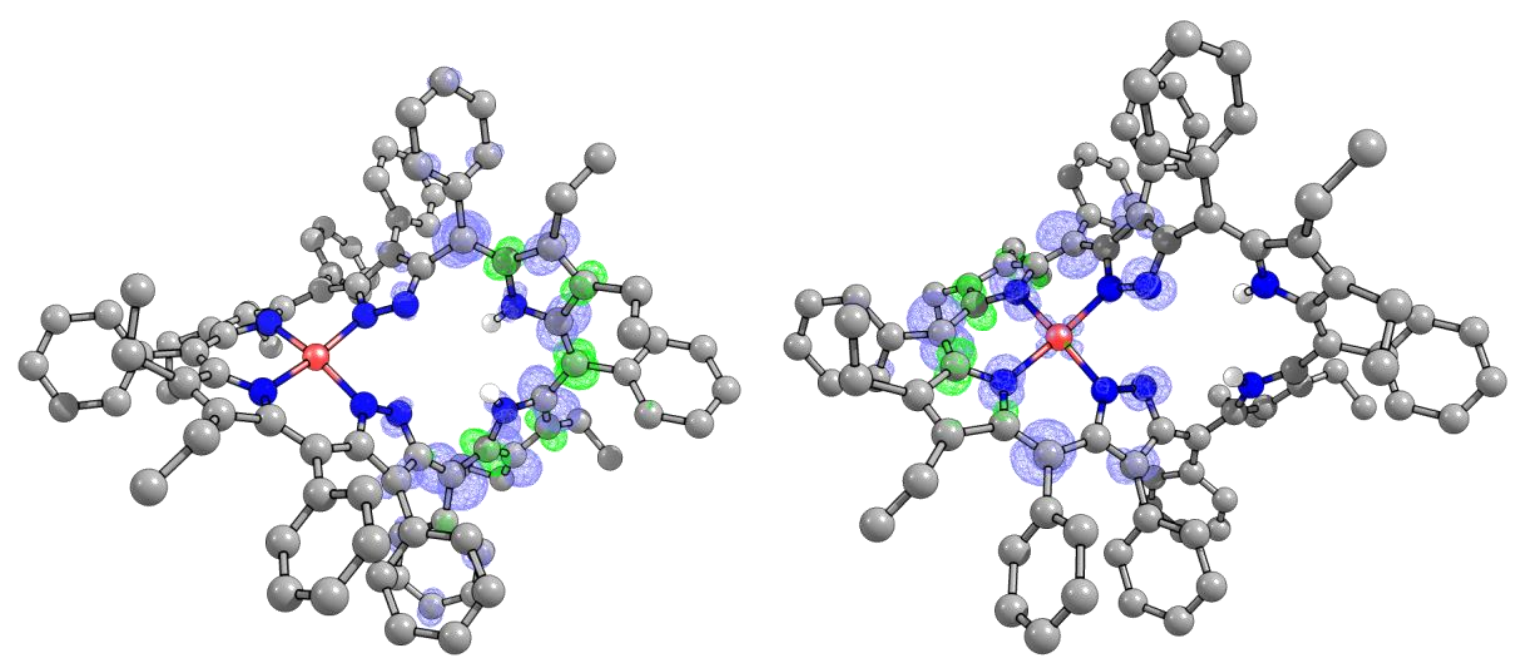

Figure 53: Visualization of spin density distribution (blue: positive, green: negative; contour value: 0.003 ) according to single point calculations for reduction and oxidation products $\mathbf{2 7} \mathbf{H}_{2} \mathbf{P d} \mathbf{d}^{-}$(left) and $\mathbf{2 7} \mathbf{H}_{2} \mathbf{P d} \mathbf{d}^{+}$(right) with cis-positioning of NH-moieties. Carbon-bound hydrogen atoms omitted for clarity. Corresponding calculated values are listed in Chapter 6.3. 
In summary, the different approaches using complementary methods for the investigation of

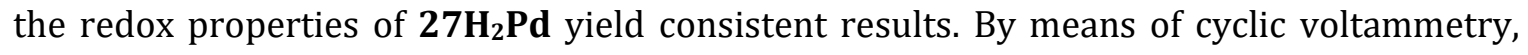
spectroelectrochemistry, EPR spectroscopy, and DFT calculations the split electronic nature of the macrocycle $\mathbf{2 7} \mathbf{H}_{\mathbf{4}}$ could be confirmed. Although the electronic separation of both hemicycles by the pyrazole moiety in $\mathbf{2 7} \mathbf{H}_{2} \mathbf{P d}$ cannot be overcome, coordination of the metal ion plays a crucial role and establishes an indirect communication between both halves of the molecule. Palladium insertion in one coordination site induces a NH-tautomerism in the second coordination site and in consequence impacts the ligand-based redox behavior of both hemicycles of the Siamese-twin porphyrin backbone. 



\section{CONCLUSION}

The aim of this work was the study of the redox behavior of the Siamese-twin porphyrin and its mono- and bimetallic complexes and the development of new synthetic strategies towards a fully macrocycle aromatic system.

The first strategy included the two- and four-electron oxidation of the free-base Siamese-twin porphyrin framework $\mathbf{2 7 \mathbf { H } _ { 4 }}$ and revealed the preferred formation of two successive $\mathrm{Npz}^{\mathrm{pz}}$ - ortho-Ph linkages as seen in products $\mathbf{3 2} \mathbf{H}_{3}$ and $\mathbf{3 3 H}_{\mathbf{2}}$ over the expression of a $26 \pi$ electron-bearing macrocycle (Figure 54). The N-fusion products could be spectroscopically and structurally characterized, with good agreement between the solution and the solid-state structures. The oxidative fusion reaction is remarkable in several regards: First, the linkages form pyrazolo[1,5- $a$ ] indole moieties that are incorporated into the macrocyclic structure and represent novel building blocks in expanded porphyrins. Second, the fusion involves a pyrazole nitrogen and an unactivated phenyl-CH group. Third, the fusions extend the $\pi$-conjugation pathways of the two independent conjugated $\pi$-systems present in the
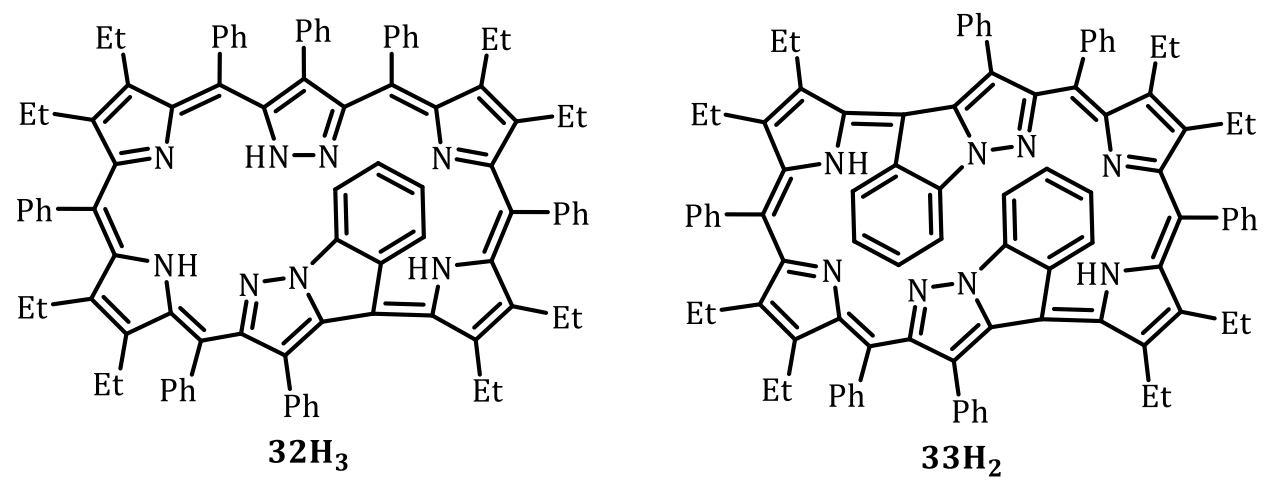

Figure 54: Products of successive one and two $\mathrm{Npz}_{-}$- ortho-Ph linkages $\left(32 \mathbf{H}_{3}\right.$ and $\left.33 \mathbf{H}_{2}\right)$ upon two- and four-electron oxidation of the free-base Siamese-twin porphyrin $\mathbf{2 7} \mathbf{H}_{4}$.

Siamese-twin porphyrin, but do not link them. Consequently, macrocycle aromatic molecules based on the Siamese-twin porphyrin backbone remain elusive. Finally, the oxidative fusion reactions led to more and more twisted and eventually folded-over topologies. The first linkage site seems to be controlled by the conformation of the precursor macrocycle, but the second - chemically equivalent fusion - required a major rearrangement of the macrocycle by a movement best described as an eversion, ultimately forming a boxlike structure in which the formerly inward-pointing pyrazole nitrogen atoms are now pointing outwards. There is no evidence for any release of strain from the parent twisted structures. The fusions between non-activated Cortho-Ph positions and the pyrazole NH stand in contrast to similar intramolecular ring fusions in expanded porphyrins.

The second strategy included the synthesis of modified key building blocks to circumvent the interruption of the global conjugation pathway and macrocyclic distortion present in the Siamese-twin porphyrin $\mathbf{2} \mathbf{7 H}_{4}$ and caused by the pyrazole moiety. Variations in the substitution pattern and introduction of triazole as an alternative merging heterocycle were pursued as rational alternatives (Figure 55). While the synthesis of suitable triazole derivatives was successfully adapted from literature, conceivable conversions with 
substituted and unsubstituted pyrroles towards the pyrrole/triazole-hybrid (42) proved ineffective. In turn, synthesis of a sterically less crowded 4-unsubstituted pyrazole (46)although feasible-remained unaccomplished due to obstructed availability of starting materials and troubled purification of the desired products (mixture of 46 and 49).<smiles>[R]c1c[nH]c(Cc2nnc(Cc3[nH]cc([R])c3[R])n2[R])c1[R]</smiles><smiles>O=c1[nH][nH]c(Cc2ccccc2)c1-c1ccccc1</smiles>

Figure 55: Aspired key building blocks in the syntheses towards sterically and electronically modified Siamesetwin porphyrins: A) pyrrole/triazole-hybrid (42) and B) 4-unsubstituted pyrazole (46).

Further investigations involved the combination of the Siamese-twin porphyrin as a redox non-innocent ligand with manganese as a multi-valent metal ion. Homo- and heterobimetallic complexes 27Mn(III)Mn(III) and 27 Ni(II) Mn(III) with varying axial ligands (acetate and azide; Figure 56) could be synthesized and spectroscopically and structurally characterized. Solid state measurements (X-ray and SQUID) provided evidence for a formal $\mathrm{d}^{4}$-Mn(III) high-spin electronic configuration with an antiferromagnetic interaction of the manganese centers. However, measurements in solution revealed a more complex and dynamic nature of metalligand interaction. The dynamic charge delocalization between the redox non-innocent ligand framework and the redox active and coordinationally flexible manganese ions requires further detailed analyses such as variable temperature HF-EPR measurements to probe the electronic state of the manganese ions and MCD spectroscopy to uncover the molecular origin of UV/vis-NIR transitions characteristic for the $\mathbf{2 7} \mathbf{M n}_{\mathbf{2}}$ species.
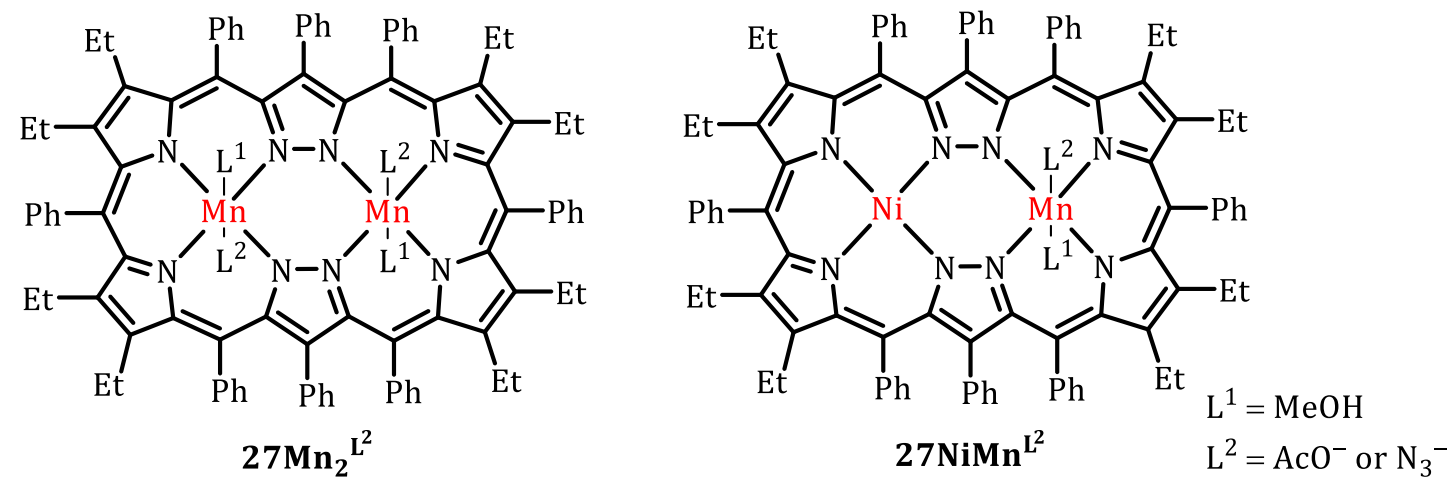

Figure 56: Homo- and heterobimetallic complexes $27 \mathbf{M n}_{2}{ }^{\mathbf{L}^{2}}$ and $\mathbf{2 7} \mathbf{N i M n}{ }^{\mathbf{L}^{2}}$ with varying axial ligands.

Finally, to find the balance between the two-electron oxidation chemistry of the free-base ligand $\mathbf{2 7} \mathbf{H}_{4}$ and the complex (multi-)electron nature of the dimanganese complexes $\mathbf{2 7} \mathbf{M n}_{2}$, a complex of the redox-inert palladium(II) ion $\left(\mathbf{2} \mathbf{7} \mathbf{H}_{2} \mathbf{P d}\right)$ was synthesized. The Siamese-twin porphyrin scaffold proved sufficiently flexible to incorporate the larger $4 \mathrm{~d}$-metal ion in a square-planar geometry. The LEWIS-acidity of the coordinated metal ion evoked a crucial NHproton tautomerism that was observed in both solid state and in solution. Electrochemical investigations revealed the split nature of the macrocyclic backbone: even though the first one-electron oxidation and reduction processes occur within the conjugated ligand framework, they differ in their choice of ligand sites (Figure 57). While the reduction prefers 

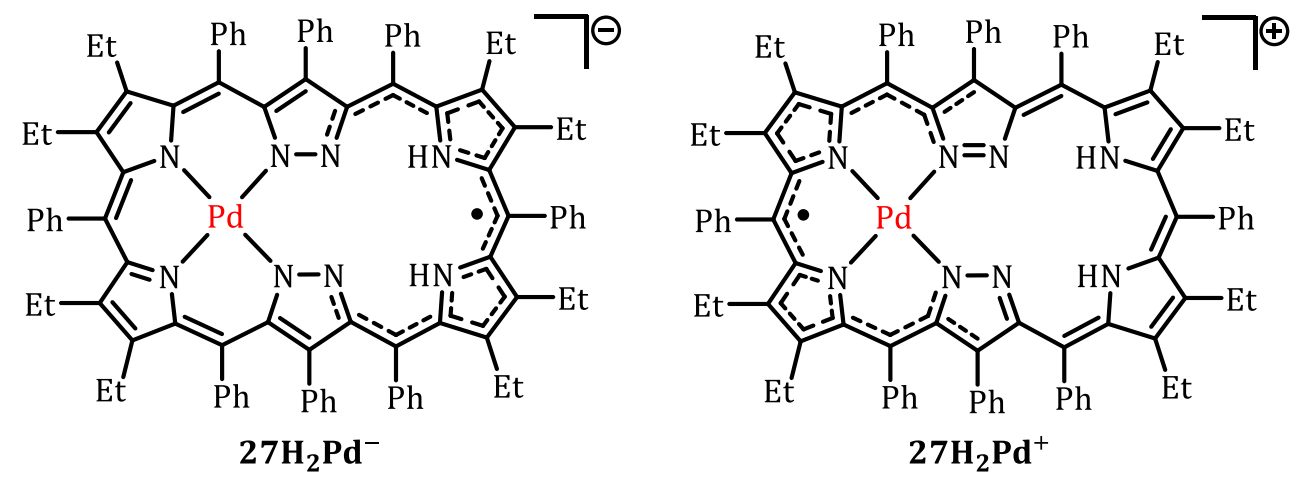

Figure 57: The split nature of the macrocyclic ligand framework in complex $\mathbf{2 7} \mathbf{H}_{2} \mathbf{P d}$ : While the reduction takes place at the neutral metal-free site of the molecule $\left(2 \mathbf{2 H}_{2} \mathbf{P d} \mathbf{d}^{-}\right)$, the oxidation occurs on the metal-coordinating, formally doubly charged coordination pocket $\left(\mathbf{2 7 H}_{2} \mathbf{P} \mathbf{d}^{+}\right)$. Spin delocalization of the $\pi$-radical anion and cation is indicated with dashed lines.

the neutral metal-free site of the molecule $\left(\mathbf{2 7} \mathbf{H}_{2} \mathbf{P d} \mathbf{d}^{-}\right)$, the oxidation takes place on the metalcoordinating, formally doubly charged coordination pocket $\left(\mathbf{2 7}_{2} \mathbf{P} \mathbf{d}^{+}\right)$. DFT calculations revealed that this redox behavior is highly enabled by the palladium-coordination induced proton shift in the metal-free coordination site. Consequently, although no macrocycle-wide conjugation is present in the Siamese-twin porphyrin framework, both ligand subunits still have enormous impact on each other's electronic structures and behavior.

The presented findings on the redox properties of the Siamese-twin porphyrin and its monoand bimetallic complexes contribute greatly to today's understanding of the ever-growing yet still young family of expanded porphyrins. All spectroscopic and structural characterizations reveal that even though the connectivity of the Siamese-twin porphyrin with its six mesolinked subunits resembles the shape of hexaphyrins, it seems that its electronic structure is much more closely related to the figure-eight non-aromatic octaphyrins. The split nature of the $\pi$-system in the Siamese-twin porphyrin must not be considered a handicap but embraced as an opportunity that further expands the potential of Siamese-twin porphyrins to serve as an adjustable platform for multi-electron redox processes in chemical catalysis or molecular electronics applications. 



\section{EXPERIMENTAL SECTION}

\subsection{Methods}

\section{NMR Spectroscopy}

${ }^{1} \mathrm{H}$ and ${ }^{13} \mathrm{C}$ NMR spectra were recorded on a BRUKER AVANCE $500 \mathrm{MHz}$ spectrometer. Chemical shifts $(\delta)$ are reported in ppm relative to residual proton and the carbon signals of $\mathrm{CD}_{2} \mathrm{Cl}_{2}$ at $5.32 \mathrm{ppm}$ and $54.00 \mathrm{ppm}$, respectively. ${ }^{13} \mathrm{C}$ NMR spectra were recorded in proton-decoupled mode. 2D NMR techniques $\left({ }^{1} \mathrm{H}^{1} \mathrm{H}-\mathrm{COSY},{ }^{1} \mathrm{H}^{13} \mathrm{C}-\mathrm{HSQC},{ }^{1} \mathrm{H}^{13} \mathrm{C}-\mathrm{HMBC},{ }^{1} \mathrm{H}^{15} \mathrm{~N}-\mathrm{HMBC}\right.$, NOESY) were recorded accordingly. Multiplicities of the signals are abbreviated as follows: s (singlet), $d$ (doublet), t (triplet), q (quartet), m (multiplet) and sbr (broad singlet). Coupling constants $J$ are given in $\mathrm{Hz}$.

\section{Mass Spectrometry}

ESI MS measurements were recorded on an APPLIED BIOSYSTEMS API 2000 spectrometer. HR-MS measurements were recorded on a BRUKER MAXIS spectrometer with $\mathrm{MeOH}$ as a carrier solvent. MALDI HR-MS measurements were recorded on a BRUKER AUTOFLEX SPEED spectrometer using a DCTB matrix and poly(methyl methacrylate) (PMMA) as an internal standard.

\section{UV/vis and Infrared Spectroscopy}

UV/vis spectra were recorded with AGILENT CARY 60 or AGILENT CARY 5000 spectrometers using quartz cuvettes $(d=1 \mathrm{~cm})$ in the solvents indicated. For measurements under inert conditions and at variable temperatures, SCHLENK cuvettes were used. For temperature controlled measurements, an UNISOKU COOLSPEK UV/CD cryostat was mounted as a sample holder.

IR spectra were recorded on an AGILENT CARY 630 FTIR spectrometer (ATR measurements using solid material) and processed with MICROLAB FTIR software. The peaks ( $\tilde{v}$ ) are labelled according to their intensity: vs (very strong), s (strong), m (medium), w (weak), vw (very weak).

\section{Cyclic voltammetry}

Cyclic voltammograms were recorded under inert conditions using a PERKINELMER 263A potentiostat. The samples were measured in $\mathrm{CH}_{2} \mathrm{Cl}_{2}$ solutions with $\mathrm{Bu}_{4} \mathrm{NPF}_{6}(0.1 \mathrm{M})$ as conductive salt. A glassy carbon electrode served as the working electrode, a platinum wire as a counter electrode and a silver wire as a pseudo-reference electrode. Decamethylferrocene $\left(\mathrm{FeCp}_{2}{ }_{2}\right)$ was added as an internal standard and all spectra were referenced to the ferrocene/ferrocenium redox couple $\left(\mathrm{Fc} / \mathrm{Fc}^{+}\right)$with $E^{0}\left(\mathrm{FeCp}_{2}{ }_{2}\right)=-590 \mathrm{mV}$ vs $\mathrm{Fc} / \mathrm{Fc}^{+}\left(E_{1 / 2}\left(\mathrm{FeCp}_{2}^{*}\right)=-0.07 \mathrm{~V}\right.$ at given conditions $) .{ }^{[142]}$

Bulk electrolysis in solutions was performed in a glovebox using a platinum net (surface area: $A \approx 1 \mathrm{~cm}^{2}$ ) as working electrode. All other conditions were adopted from cyclic voltammetry. 


\section{Magnetic Susceptibility Measurements}

Susceptibility measurements were carried out with a QUANTUM-DESIGN MPMS XL-5 SQUID magnetometer equipped with a 5 Tesla magnet. The powdered sample was contained in a gel bucket and fixed in a non-magnetic sample holder. The samples were measured in the temperature range from $300 \mathrm{~K}$ to $2 \mathrm{~K}$. Each raw data file for the measured magnetic moment was corrected for the diamagnetic contribution of the sample holder and the gel bucket. The molar susceptibility data were corrected for the diamagnetic contribution and temperatureindependent paramagnetism (TIP) according to $\chi_{\text {calc }}=\chi+$ TIP. Magnetic properties were simulated using the JULX program[119] with a spin Hamiltonian operator considering exchange coupling, zero-field splitting and Zeemann interactions.

\section{Electron Paramagnetic Resonance}

EPR spectra were recorded at $150 \mathrm{~K}$ on a BRUKER ELEXSYS E500 spectrometer, equipped with an ER $049 \mathrm{X}$ microwave bridge, an ER 083 CS magnet and a temperature control system ER 4131 VT with liquid nitrogen as cooling agent. The microwave frequency $(9.4 \mathrm{GHz}$, $\mathrm{X}$ band) was measured with a HEWLETT-PACKARD frequency counter (HP5352B). The spectra were fitted using the program ESIM.[138,139]

\section{Photolysis}

As a UV light source for irradiation of the $\mathrm{KBr}$ pellet a LOT-QUANTUMDESIGN xenon lamp (LSE140/160.25C, 150 W) equipped with an optical glass filter (280FS10-50) was used. The pellet was mounted into the IR sample holder and exposed to the UV radiation under aerobic conditions.

\section{Crystal Structure Analysis}

$\mathrm{X}$-ray data were collected on a STOE IPDS II diffractometer (graphite monochromated Mo-K $\alpha$ radiation, $\lambda=0.71073 \AA$ ) by use of scans at $-140^{\circ} \mathrm{C}$ (see also X-ray Crystallographic Details). The data were corrected towards LORENTZ- and polarization effects. The structures were solved by direct methods (SHELXS-97 or SHELXS-2013/14) and refined on $\mathrm{F}^{2}$ using all reflections with SHELXL-2013/14.[143] Crystallographic details and selected bond lengths and angles are listed in the Chapter 6.1.

\section{Materials}

Unless otherwise noted, materials obtained from commercial suppliers were used without further purification. Thin layer chromatography (TLC) was carried out on fluorescence active polyester sheets coated with silica gel MACHERY-NAGEL MN60 (Polygram ${ }^{\circledR}$ SIL G/UV254). Preparative chromatography was carried out on fluorescent glass-backed alumina plates (FLUKA, $250 \mu \mathrm{m}, 11.0-14.0 \mu \mathrm{m}$ ). Synthesis of air- or moisture- sensitive compounds was carried out under an atmosphere of dry nitrogen in SCHLENK flasks or in a glovebox. Anhydrous solvents were freshly distilled under an atmosphere of dry nitrogen according to standard procedures.[144] Compounds 3,4-diethylpyrrole,[145] 3,5-dibenzoyl-4-phenyl-1H- 
pyrazole (28),[78] and the Siamese-twin porphyrinogen $\left(\mathbf{2 9 H}_{\mathbf{6}}\right)^{[75]}$ were synthesized according to reported methods. Modified protocols are reported in detail further below.

\subsection{Syntheses}

\subsubsection{3,5-Dibenzoyl-4-phenyl-1H-pyrazole 28}<smiles>O=C(c1ccccc1)c1n[nH]c(C(=O)c2ccccc2)c1-c1ccccc1</smiles>

To a solution of 1,3-diphenylpropane-1,3-dione (3.2 g, $14 \mathrm{mmol}, 1 \mathrm{eq})$ in $\mathrm{MeOH}$ (200 mL) were added 2-diazo-1,3-diphenylpropane-1,3-dione ( $4.7 \mathrm{~g}$, $19 \mathrm{mmol}, 1.4 \mathrm{eq}$ ) and $\mathrm{K}_{2} \mathrm{CO}_{3}$ (3.9 g, $28 \mathrm{mmol}, 2 \mathrm{eq})$. The mixture was cooled with an ice bath to $0{ }^{\circ} \mathrm{C}$. After stirring for $2 \mathrm{~d}\left(0^{\circ} \mathrm{C} \rightarrow\right.$ r.t.) the solvent volume was reduced to a minimum $(\sim 25 \mathrm{~mL})$ and $\mathrm{H}_{2} \mathrm{O}(50 \mathrm{~mL})$ was added. The mixture was stirred overnight. The precipitated 3,5-dibenzoyl-4-phenyl-1H-pyrazole potassium salt was filtered off, washed with $n$-hexane and dried in vacuo. The obtained solid was subsequently suspended in a mixture of $\mathrm{Et}_{2} \mathrm{O} / \mathrm{H}_{2} \mathrm{O}(1: 1$, each $50 \mathrm{~mL})$, acidified with $\mathrm{HCl}$ (conc., $5 \mathrm{~mL}$ ) and the aqueous layer was extracted repeatedly with $\mathrm{Et}_{2} \mathrm{O}(2 \times 50 \mathrm{~mL})$. The organic phase was dried over $\mathrm{MgSO}_{4}$ and the solvent was removed in vacuo yielding 28 as a white powder (1.4 g, $41 \%$ ).

All analytical data agreed well with previously reported literature.[78]

\subsubsection{3,5-Bis-(hydroxy(phenyl)methyl-4-phenyl-1H-pyrazole 50}<smiles>OC(c1ccccc1)c1n[nH]c(C(O)c2ccccc2)c1-c1ccccc1</smiles>

(50)

To a solution of 3,5-dibenzoyl-4-phenyl- $1 \mathrm{H}$-pyrazole (28) (3.00 g, $8.51 \mathrm{mmol}, 1 \mathrm{eq})$ in $\mathrm{CHCl}_{3}$ $(10 \mathrm{~mL})$ and $\mathrm{MeOH}(5 \mathrm{~mL}), \mathrm{NaBH}_{4}(0.64 \mathrm{mg}, 17.0 \mathrm{mmol}, 2 \mathrm{eq})$ was added slowly under ice cooling, and stirred for $4 \mathrm{~h}$. Reaction progress was monitored by TLC. After addition of $\mathrm{MeOH}$ $(30 \mathrm{~mL})$, the solution was washed twice with aqueous $\mathrm{NH}_{4} \mathrm{Cl}$ solution $(50 \mathrm{~mL}, 100 \mathrm{mM}$ ), the organic phase was dried over $\mathrm{MgSO}_{4}$ and the solvent was removed under reduced pressure. The crude material was purified by column chromatography (silica, $n$-hexane/EtOAc 1:2) yielding 50 as a white powder $(2.41 \mathrm{~g}, 79 \%)$. The crude, non-chromatographed product can be used for the conversion to $\mathbf{5 1}$.

All analytical data agreed well with previously reported literature. $[76,80,84]$ 
5.2.3 3,5-Bis-(chloro(phenyl)methyl)-4-phenyl-1H-pyrazole hydrochloride $\mathbf{5 1}$<smiles>ClC(c1ccccc1)c1[nH]nc(-c2ccccc2)c1C(Cl)c1ccccc1</smiles>

(51)

Under inert conditions, to 3,5-bis-(hydroxy(phenyl)methyl-4-phenyl-1H-pyrazole (50) ( $3.00 \mathrm{~g}, 8.42 \mathrm{mmol}$ ) a molar excess of freshly distilled $\mathrm{SOCl}_{2}$ was added. The mixture was stirred at r.t. for $3 \mathrm{~d}$. Residual $\mathrm{SOCl}_{2}$ was removed under reduced pressure at r.t. The crude product was dissolved in anhydrous toluene and the solvent was subsequently removed under reduced pressure at r.t. The resulting pale yellow, moisture sensitive solid (3.20 g, $95 \%$ ) was stored under anhydrous argon atmosphere.

All analytical data agreed well with previously reported literature.[76,84]

\subsubsection{3,5-Bis-(3,4-diethyl-1H-pyrrole-2-yl-benzyl)-1H-pyrazole 29}<smiles>CCc1c[nH]c(C(c2ccccc2)c2n[nH]c(C(c3ccccc3)c3[nH]cc(CC)c3CC)c2-c2ccccc2)c1CC</smiles>

Under inert conditions, to a solution of 3,5-bis-(chloro(phenyl)methyl)-4-phenyl- $1 \mathrm{H}$ pyrazole hydrochloride (51) $(1.00 \mathrm{~g}, 2.33 \mathrm{mmol}, 1 \mathrm{eq})$ in anhydrous $\mathrm{CH}_{2} \mathrm{Cl}_{2}(60 \mathrm{~mL})$ 3,4-diethylpyrrole (41) (0.95 g, $7.71 \mathrm{mmol}, 3.5 \mathrm{eq}$ ) was added dropwise, and the reaction mixture was stirred for $2 \mathrm{~h}$ at r.t. The reaction progress was monitored by TLC. The mixture was washed with $\mathrm{H}_{2} \mathrm{O}(50 \mathrm{~mL})$, and the organic layer was separated. The aqueous layer was extracted with $\mathrm{CH}_{2} \mathrm{Cl}_{2}(2 \times 20 \mathrm{~mL})$. The combined organic layers were dried over $\mathrm{MgSO}_{4}$ and the solvent was removed in vacuo. The crude material was purified by column chromatography (silica, $n$-hexane/EtOAc 5:1) yielding a orange-brown solid ( $0.50 \mathrm{~g}, 41 \%)$. The product is prone to decomposition and has to be kept under inert conditions in the freezer.

All analytical data agreed well with previously reported literature.[76,84] 


\subsubsection{Siamese-Twin Porphyrinogen $\mathbf{3 1 H _ { 6 }}$}

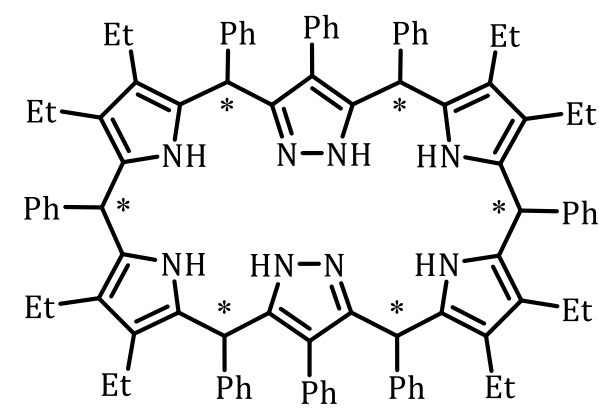

$\left(31 \mathrm{H}_{6}\right)$

Benzaldehyde ( $180 \mu \mathrm{L}$ of a $1 \mathrm{~m}$ solution in $\mathrm{CH}_{2} \mathrm{Cl}_{2} ; 19.1 \mathrm{mg}, 0.18 \mathrm{mmol}, 1 \mathrm{eq}$ ) and TFA ( $180 \mu \mathrm{L}$ of a $1 \mathrm{M}$ solution in $\mathrm{CH}_{2} \mathrm{Cl}_{2} ; 20.5 \mathrm{mg}, 0.18 \mathrm{mmol}, 1$ eq) were diluted with $\mathrm{CH}_{2} \mathrm{Cl}_{2}(14.9 \mathrm{~mL})$ and protected from light. 3,5-Bis-(3,4-diethyl- $1 H$-pyrrole-2-yl-benzyl)- $1 H$-pyrazole (29) $(180 \mu \mathrm{L}$ of a $1 \mathrm{M}$ solution in $\mathrm{CH}_{2} \mathrm{Cl}_{2} ; 100 \mathrm{mg}, 0.18 \mathrm{mmol}, 1 \mathrm{eq}$ ) was added and stirred for $2 \mathrm{~h}$. The reaction progress was monitored by TLC. The solvent volume was reduced to a minimum $(<5 \mathrm{~mL})$ and the orange-pink solution was run through a short column of basic alumina (BRoCKMANN grade I, 50-200 $\mu \mathrm{m}$ ) with pure $\mathrm{CH}_{2} \mathrm{Cl}_{2}$ as an eluent. After removal of solvent, product $\mathbf{3 1}_{6}$ was obtained as an orange solid $(70.7 \mathrm{mg}, 60 \%)$.

All analytical data agreed well with previously reported literature.[76,84]

\subsubsection{Siamese-Twin Porphyrin $\mathbf{2 7 \mathbf { H } _ { 4 }}$}

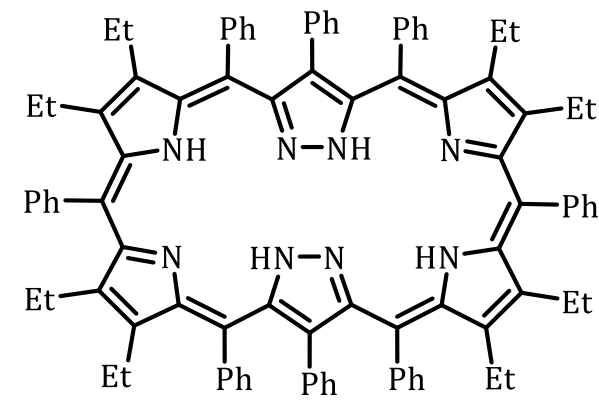

$\left(27 \mathrm{H}_{4}\right)$

Siamese-twin Porphyrin $\mathbf{2 7} \mathbf{H}_{\mathbf{4}}$ was prepared according to literature, $[76,78,80]$ except that the work-up was altered as follows: The solvent was removed in vacuo from the crude reaction mixture and the residue was flushed through a short column of basic alumina (BROCKMANN grade I, 50-200 $\mu \mathrm{m})$ with THF/ $\mathrm{CH}_{2} \mathrm{Cl}_{2} / \mathrm{EtOAc}(10: 3: 1)$ as eluent. The first teal colored fraction was collected and the solvent was removed in vacuo. The crude product was taken up in a minimal amount of cold acetone and filtered (yield: $21 \%$ ). Subsequent solvent removal of the filtrate followed by a SEPHADEX column with $\mathrm{MeOH}$ as eluent provided spectroscopically pure $\mathbf{2 7 H}_{\mathbf{4}}$. Single crystals were obtained by slow evaporation of a saturated benzene solution. All analytical data agree well with reported literature. Herein a more detailed assignment of the spectra of $\mathbf{2 7} \mathbf{H}_{4}$ is provided: 
TLC:

$R_{\mathrm{f}}=0.54$ (n-hexane/THF/EtOAc 20:3:1);

${ }^{1} \mathrm{H}$ NMR (500 MHz, $\left.\mathrm{CD}_{2} \mathrm{Cl}_{2}, 238 \mathrm{~K}\right): \quad \delta(\mathrm{ppm})=13.36\left(\mathrm{~s}, 2 \mathrm{H}, \mathrm{Np}^{\mathrm{p}} \mathrm{H}\right), 11.37(\mathrm{~s}, 2 \mathrm{H}, \mathrm{Npy}), 7.53$ (dd, $J=13.7,7.4 \mathrm{~Hz}, 4 \mathrm{H}, \mathrm{Ph}$ ), $7.35-7.23$ (m, 8H, Ph), 7.20 (dtd, $J=15.4,8.2,7.4,4.4 \mathrm{~Hz}, 4 \mathrm{H}, \mathrm{Ph}), 7.06(\mathrm{~d}, J=7.1 \mathrm{~Hz}, 2 \mathrm{H}$, $\mathrm{Ph}$ ), 7.02 (d, J=7.4 Hz, 2H, Ph), $6.97(\mathrm{t}, J=7.5 \mathrm{~Hz}, 2 \mathrm{H}, \mathrm{Ph}$ ), $6.85(\mathrm{~d}, J=7.7 \mathrm{~Hz}, 2 \mathrm{H}, \mathrm{Ph}), 6.82-6.78(\mathrm{~m}, 2 \mathrm{H}, \mathrm{Ph})$, $6.78-6.74(\mathrm{~m}, 2 \mathrm{H}, \mathrm{Ph}), 6.56(\mathrm{t}, J=7.6 \mathrm{~Hz}, 2 \mathrm{H}, \mathrm{Ph})$, $6.33-6.26(\mathrm{~m}, 2 \mathrm{H}, \mathrm{Ph}), 6.18(\mathrm{~d}, J=7.7 \mathrm{~Hz}, 2 \mathrm{H}, \mathrm{Ph}), 1.99$ (dq, $\left.J=14.3,7.2 \mathrm{~Hz}, 2 \mathrm{H}, \mathrm{CH}_{2}\right), 1.86\left(\mathrm{dq}, J=14.5,7.4 \mathrm{~Hz}, 2 \mathrm{H}, \mathrm{CH}_{2}\right)$, $1.55\left(\mathrm{dh}, J=14.4,7.0 \mathrm{~Hz}, 4 \mathrm{H}, \mathrm{CH}_{2}\right), 1.42(\mathrm{dq}, J=18.1,7.3 \mathrm{~Hz}$, $\left.2 \mathrm{H}, \mathrm{CH}_{2}\right), 1.19\left(\mathrm{dt}, J=14.4,7.1 \mathrm{~Hz}, 2 \mathrm{H}, \mathrm{CH}_{2}\right), 0.90-0.81(\mathrm{~m}$, $\left.2 \mathrm{H}, \mathrm{CH}_{2}\right), 0.55\left(\mathrm{q}, J=7.6 \mathrm{~Hz}, 12 \mathrm{H}, \mathrm{CH}_{3}\right), 0.45(\mathrm{t}, J=7.3 \mathrm{~Hz}, 6 \mathrm{H}$, $\left.\mathrm{CH}_{3}\right), 0.41\left(\mathrm{t}, J=7.3 \mathrm{~Hz}, 6 \mathrm{H}, \mathrm{CH}_{3}\right)$;

${ }^{13} \mathrm{C}$ NMR $\left(126 \mathrm{MHz}, \mathrm{CD}_{2} \mathrm{Cl}_{2}, 238 \mathrm{~K}\right): \delta(\mathrm{ppm})=167.5\left(\mathrm{C}_{\mathrm{q}}\right), 151.4\left(\mathrm{C}_{\mathrm{q}}\right), 151.1\left(\mathrm{C}_{\mathrm{q}}\right), 148.2\left(\mathrm{C}_{\mathrm{q}}\right)$, $147.6\left(\mathrm{C}_{\mathrm{q}}\right), 145.2\left(\mathrm{C}_{\mathrm{q}}\right), 141.6\left(\mathrm{C}_{\mathrm{q}}\right), 141.2\left(\mathrm{C}_{\mathrm{q}}\right), 140.6\left(\mathrm{C}_{\mathrm{q}}\right)$, $139.3\left(\mathrm{C}_{\mathrm{q}}\right), 139.1\left(\mathrm{C}_{\mathrm{q}}\right), 138.0\left(\mathrm{C}_{\mathrm{q}}\right), 135.3\left(\mathrm{C}_{\mathrm{q}}\right), 133.8\left(\mathrm{C}_{\mathrm{q}}\right)$, $133.2(\mathrm{CH}), 133.1(\mathrm{CH}), 132.6(\mathrm{CH}), 132.5(\mathrm{CH}), 132.4(\mathrm{CH})$, $131.6(\mathrm{CH}), 130.7(\mathrm{CH}), 127.9(\mathrm{CH}), 127.8(\mathrm{CH}), 127.6$ (3CH), $127.2(2 \mathrm{CH}), 127.1(\mathrm{CH}), 127.1(\mathrm{CH}), 126.5(\mathrm{CH})$, $126.5(\mathrm{CH}), 126.1(\mathrm{CH}), 124.5(\mathrm{CH}), 124.3\left(\mathrm{C}_{\mathrm{q}}\right), 124.0\left(\mathrm{C}_{\mathrm{q}}\right)$, $114.1\left(\mathrm{C}_{\mathrm{q}}\right), 106.0\left(\mathrm{C}_{\mathrm{q}}\right), 18.9\left(\mathrm{CH}_{2}\right), 18.5\left(\mathrm{CH}_{2}\right), 18.1\left(\mathrm{CH}_{2}\right)$, $18.0\left(\mathrm{CH}_{2}\right), 16.2\left(\mathrm{CH}_{3}\right), 15.9\left(\mathrm{CH}_{3}\right), 15.6\left(\mathrm{CH}_{3}\right), 14.7\left(\mathrm{CH}_{3}\right)$;

IR (ATR):

$\tilde{v}\left(\mathrm{~cm}^{-1}\right)=2973(\mathrm{w}), 2928(\mathrm{w}), 2869(\mathrm{w}), 1563(\mathrm{w}), 1514$ (m), $1488(\mathrm{~m}), 1444(\mathrm{w}), 388(\mathrm{~m}), 1358(\mathrm{~m}), 1317$ (vw), 1298 (vw), 1257 (w), 1209 (vw), 1190 (vw), 1175 (vw), 1138 (m), 1115 (m), 1052 (m), 1030 (m), 1015 (m), 981 (vw), $951(\mathrm{w}), 918(\mathrm{vw}), 888(\mathrm{w}), 873(\mathrm{w}), 802(\mathrm{~m}), 768$ (m), $750(\mathrm{w}), 739(\mathrm{~m}), 694(\mathrm{vs}), 671(\mathrm{w}), 638(\mathrm{~m}), 615(\mathrm{w})$, $582(\mathrm{w}), 548(\mathrm{vw}), 526(\mathrm{vw}), 511(\mathrm{w}), 466(\mathrm{vw}), 444(\mathrm{vw})$;

$\mathrm{UV} /$ vis $\left(\mathrm{CH}_{2} \mathrm{Cl}_{2}\right)$ : $\lambda_{\max }\left(\mathrm{nm} ; \varepsilon\left[\mathrm{L} \mathrm{mol}^{-1} \mathrm{~cm}^{-1}\right]\right)=305$ (29 400), 389 (76 800), 641 (34 200), 740 (13 400);

$\mathrm{MS}\left(\mathrm{ESI}^{+}, \mathrm{MeOH}\right)$ :

$m / z(\%)=1302(100)\left[\mathbf{2 7}_{\mathbf{4}}+\mathrm{H}\right]^{+}, 1324(9)\left[\mathbf{2 7} \mathbf{H}_{4}+\mathrm{Na}\right]^{+} ;$

MS (ESI- $\left.{ }^{-}, \mathrm{MeOH}\right)$ :

$m / z(\%)=1300(100)\left[27 \mathbf{H}_{4}-\mathrm{H}\right]^{-}$;

HR-MS (ESI $\left.{ }^{+}, \mathrm{MeOH}\right)$ :

$m / z(\%)=1301.6892$ (calc. 1301.6892 for $\mathrm{C}_{92} \mathrm{H}_{85} \mathrm{~N}_{8}{ }^{+}$). 


\subsubsection{Mono-fused Siamese-Twin Porphyrin $\mathbf{3 2} \mathbf{H}_{3}$}

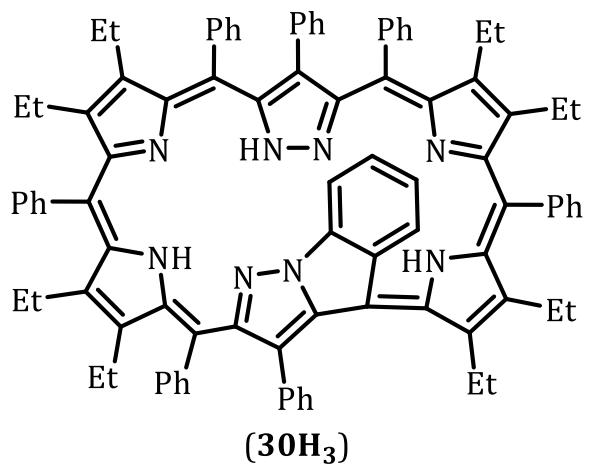

To a solution of Siamese-twin porphyrin $\mathbf{2 7 H _ { 4 }}$ (50 mg, $\left.38.4 \mu \mathrm{mol}, 1 \mathrm{eq}\right)$ in anhydrous $\mathrm{CH}_{2} \mathrm{Cl}_{2}$ ( $40 \mathrm{~mL}$ ), DDQ ( $8.7 \mathrm{mg}, 38.4 \mu \mathrm{mol}, 1 \mathrm{eq}$ ) was added all at once under inert conditions and the mixture was heated to reflux for $48 \mathrm{~h}$. The reaction progress was monitored by TLC (silica, $n$-hexane/THF/EtOAc 20:3:1, $\left.R_{\mathrm{f}}\left(\mathbf{2} \mathbf{2} \mathbf{H}_{4}\right)=0.54, R_{\mathrm{f}}\left(\mathbf{3 2 H}_{3}\right)=0.76\right)$. The solvent was removed in vacuo. The crude solid residue was purified by preparative TLC (neutral alumina, BROCKMANN grade I, 50-200 $\mu \mathrm{m}, n$-hexane/THF/EtOAc 20:3:1, $\left.R_{\mathrm{f}}\left(\mathbf{3 2 H}_{3}\right)=0.76\right)$, followed by a SEPHADEX column with $\mathrm{MeOH}$ as eluent. The product was obtained as a dark green solid (6.3 mg, $13 \%$ ). Single crystals were obtained by slow evaporation of a saturated acetone solution.

TLC:

$R_{\mathrm{f}}=0.76$ (n-hexane/THF/EtOAc 20:3:1);

${ }^{1} \mathrm{H}$ NMR (500 MHz, $\left.\mathrm{CD}_{2} \mathrm{Cl}_{2}, 248 \mathrm{~K}\right): \quad \delta(\mathrm{ppm})=15.62(\mathrm{~s}, 1 \mathrm{H}, \mathrm{Npz}), 14.07$ (s, 1H, NpyH), 11.26 (s, 1H, NpyH), 7.76 (d, J= $\left.7.9 \mathrm{~Hz}, 1 \mathrm{H}, \mathrm{Ph}^{\text {indole }}\right), 7.74-7.64(\mathrm{~m}$, 2H, Phindole), 7.57 (d, J=7.6 Hz, 1H, Phindole), $7.54-7.22$ (m, 15H, Ph), 7.22 - 6.75 (m, 9H, Ph), $6.75-6.65$ (m, 2H, Ph), $6.64-6.54(\mathrm{~m}, 5 \mathrm{H}, \mathrm{Ph}), 6.50(\mathrm{ddd}, J=8.8,6.5,1.7 \mathrm{~Hz}, 1 \mathrm{H}$, $\mathrm{Ph}), 6.44(\mathrm{~d}, J=8.1 \mathrm{~Hz}, 1 \mathrm{H}, \mathrm{Ph}), 6.31(\mathrm{td}, J=7.4,1.9 \mathrm{~Hz}, 1 \mathrm{H})$, $6.10-6.03(\mathrm{~m}, 1 \mathrm{H}, \mathrm{Ph}), 5.98(\mathrm{td}, J=7.6,1.4 \mathrm{~Hz}, 1 \mathrm{H}, \mathrm{Ph}), 5.59$ (d, $J=7.7 \mathrm{~Hz}, 1 \mathrm{H}, \mathrm{Ph}), 5.36(\mathrm{dt}, J=7.3,1.8 \mathrm{~Hz}, 1 \mathrm{H}, \mathrm{Ph}$ ), 3.03 (dq, $J=14.9,7.4 \mathrm{~Hz}, 1 \mathrm{H},{ }^{\mathrm{a}} \mathrm{CH}_{2}$ ), $2.68(\mathrm{dq}, J=14.7,7.4 \mathrm{~Hz}, 1 \mathrm{H}$, $\left.{ }^{\mathrm{a}} \mathrm{CH}_{2}\right), 2.26\left(\mathrm{dq}, J=14.6,7.4 \mathrm{~Hz}, 1 \mathrm{H}, \mathrm{C}^{\mathrm{b}} \mathrm{H}_{2}\right), 2.00(\mathrm{dt}, J=13.6$, $\left.6.9 \mathrm{~Hz}, 1 \mathrm{H}, \mathrm{b} \mathrm{CH}_{2}\right), 1.91$ (td, $J=11.8,9.8,4.9 \mathrm{~Hz}, 1 \mathrm{H},{ }^{\mathrm{c}} \mathrm{CH}_{2}$ ), $1.82\left(\mathrm{dt}, J=14.2,7.3 \mathrm{~Hz}, 1 \mathrm{H},{ }^{\mathrm{c}} \mathrm{CH}_{2}\right.$ ), 1.68 (tdd, $J=18.8,8.4$, $\left.6.3 \mathrm{~Hz}, 4 \mathrm{H}, \mathrm{d} / \mathrm{d} / \mathrm{e} / \mathrm{f} \mathrm{CH}_{2}\right), 1.50\left(\mathrm{dq}, J=12.8,6.0 \mathrm{~Hz}, 1 \mathrm{H},{ }^{\mathrm{e}} \mathrm{CH}_{2}\right)$, $1.40\left(\mathrm{dt}, J=14.3,7.2 \mathrm{~Hz}, 1 \mathrm{H}, \mathrm{gCH}_{2}\right), 1.34-1.24(\mathrm{~m}, 2 \mathrm{H}$, $\mathrm{h} / \mathrm{h} \mathrm{CH}_{2}$ ), 1.07 (t, $\left.J=7.5 \mathrm{~Hz}, 3 \mathrm{H},{ }^{\mathrm{a}} \mathrm{CH}_{3}\right), 1.03-0.94(\mathrm{~m}, 2 \mathrm{H}$, f/g $\left.\mathrm{CH}_{2}\right), 0.66-0.58\left(\mathrm{~m}, 9 \mathrm{H}, \mathrm{b} / \mathrm{c} / \mathrm{f} \mathrm{CH}_{3}\right), 0.58-0.41(\mathrm{~m}, 9 \mathrm{H}$, $\left.\mathrm{d} / \mathrm{e} / \mathrm{h} \mathrm{CH}_{3}\right), 0.37\left(\mathrm{t}, J=7.4 \mathrm{~Hz}, 3 \mathrm{H},{ }^{\mathrm{g}} \mathrm{CH}_{3}\right)$; letters indicate correlated spin systems according to 2D NMR;

${ }^{13} \mathrm{C}$ NMR $\left(126 \mathrm{MHz}, \mathrm{CD}_{2} \mathrm{Cl}_{2}, 243 \mathrm{~K}\right): \delta(\mathrm{ppm})=169.1\left(\mathrm{C}_{\mathrm{q}}\right), 164.8\left(\mathrm{C}_{\mathrm{q}}\right), 153.8\left(\mathrm{C}_{\mathrm{q}}\right), 152.3\left(\mathrm{C}_{\mathrm{q}}\right)$, $150.5\left(\mathrm{C}_{\mathrm{q}}\right), 150.1\left(\mathrm{C}_{\mathrm{q}}\right), 147.6\left(\mathrm{C}_{\mathrm{q}}\right), 146.6\left(\mathrm{C}_{\mathrm{q}}\right), 144.3\left(\mathrm{C}_{\mathrm{q}}\right)$, $144.1\left(\mathrm{C}_{\mathrm{q}}\right), 144.1\left(\mathrm{C}_{\mathrm{q}}\right), 144.0\left(\mathrm{C}_{\mathrm{q}}\right), 143.5\left(\mathrm{C}_{\mathrm{q}}\right), 142.3\left(\mathrm{C}_{\mathrm{q}}\right)$, $142.2\left(\mathrm{C}_{\mathrm{q}}\right), 141.9\left(\mathrm{C}_{\mathrm{q}}\right), 141.7\left(\mathrm{C}_{\mathrm{q}}\right), 140.6\left(\mathrm{C}_{\mathrm{q}}\right), 139.9\left(\mathrm{C}_{\mathrm{q}}\right)$, $138.5\left(\mathrm{C}_{\mathrm{q}}\right), 137.8\left(\mathrm{C}_{\mathrm{q}}\right), 137.3\left(\mathrm{C}_{\mathrm{q}}\right), 135.9\left(\mathrm{C}_{\mathrm{q}}\right), 135.6\left(\mathrm{C}_{\mathrm{q}}\right)$, 
IR (ATR):

$134.7\left(\mathrm{C}_{\mathrm{q}}\right), 133.9\left(\mathrm{C}_{\mathrm{q}}\right), 133.2,132.8,132.4,131.8,131.4$ 131.3, 130.6, 130.4, 130.0, 128.4, 128.1, 128.0, 127.9, 127.8, $127.6,127.4,127.1,126.89,126.87,126.6,126.5,126.3$, 126.2, 125.3 (2), 125.1, 124.9, 123.9, 123.5, 123.0, 115.2 $\left(\mathrm{C}_{\mathrm{q}}\right), 114.7\left(\mathrm{C}_{\mathrm{q}}\right), 114.5\left(\mathrm{C}_{\mathrm{q}}\right), 110.2\left(\mathrm{C}_{\mathrm{q}}\right), 106.4\left(\mathrm{C}_{\mathrm{q}}\right), 101.0\left(\mathrm{C}_{\mathrm{q}}\right)$, $19.5\left(\mathrm{CH}_{2}\right), 19.2\left(\mathrm{CH}_{2}\right), 18.9\left(\mathrm{CH}_{2}\right), 18.8\left(\mathrm{CH}_{2}\right), 18.7\left(\mathrm{CH}_{2}\right)$, $18.6\left(\mathrm{CH}_{2}\right), 18.0\left(\mathrm{CH}_{2}\right), 17.8\left(\mathrm{CH}_{2}\right), 16.4\left(\mathrm{CH}_{3}\right), 16.1\left(\mathrm{CH}_{3}\right)$, $16.0\left(\mathrm{CH}_{3}\right), 15.6\left(\mathrm{CH}_{3}\right), 15.5\left(\mathrm{CH}_{3}\right), 15.2\left(\mathrm{CH}_{3}\right), 14.5\left(\mathrm{CH}_{3}\right)$;

$\tilde{v}\left(\mathrm{~cm}^{-1}\right)=2969(\mathrm{w}), 2904(\mathrm{w}), 2870(\mathrm{w}), 1735(\mathrm{w}), 1707$ (vw), 1685 (vw), 1654 (vw), 1595 (vw), 1596 (vw), 1560 (w), 1542 (w), $1523(\mathrm{vw}), 1508(\mathrm{w}), 1489$ (vw), $1458(\mathrm{w})$, 1439 (w), 1400 (vw), 1363 (w), 1346 (w), 1308 (vw), 1234 (w), 1174 (vw), 1133 (vw), 1092 (vw), 1046 (w), 1008 (w), $952(\mathrm{vw}), 941$ (vw), $883(\mathrm{w}), 844(\mathrm{vw}), 805(\mathrm{vw}), 764(\mathrm{~m})$, $738(\mathrm{~m}), 695$ (vs), $672(\mathrm{~m}), 659(\mathrm{~m})$;

$\mathrm{UV} /$ vis $\left(\mathrm{CH}_{2} \mathrm{Cl}_{2}\right)$ : $\lambda_{\max }\left(\mathrm{nm} ; \varepsilon\left[\mathrm{L} \mathrm{mol}^{-1} \mathrm{~cm}^{-1}\right]\right)=319$ (59 900), 409 (74 100), 685 (33 500), 830 (14 000);

$\mathrm{MS}\left(\mathrm{ESI}^{+}, \mathrm{MeOH}\right)$ :

$m / z(\%)=1299.7(100)\left[32 \mathbf{H}_{3}+\mathrm{H}\right]^{+} ;$

HR-MS (ESI $\left.{ }^{+}, \mathrm{MeOH}\right)$ :

$m / z(\%)=1299.6736$ (calc. 1299.6735 for $\mathrm{C}_{92} \mathrm{H}_{83} \mathrm{~N}_{8}{ }^{+}$).

\subsubsection{Bis-fused Siamese-Twin Porphyrin $\mathbf{3 3} \mathbf{H}_{2}$}

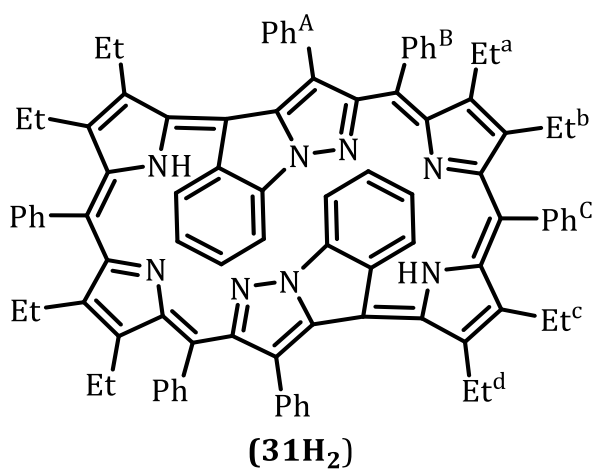

To a solution of Siamese-twin porphyrin $\mathbf{2 7 \mathbf { H } _ { 4 }}(100 \mathrm{mg}, 76.8 \mu \mathrm{mol}, 1 \mathrm{eq})$ in anhydrous $\mathrm{CH}_{2} \mathrm{Cl}_{2}$ ( $40 \mathrm{~mL}$ ), DDQ (34.8 mg, $153.6 \mu \mathrm{mol}, 2 \mathrm{eq}$ ) was added all at once under inert conditions, and the mixture was heated to reflux for $48 \mathrm{~h}$. The reaction progress was monitored by TLC (silica, $n$-hexane/THF/EtOAc 20:3:1, $\left.R_{\mathrm{f}}\left(\mathbf{2 7 H}_{4}\right)=0.54, R_{\mathrm{f}}\left(\mathbf{3 2 H}_{3}\right)=0.76, R_{\mathrm{f}}\left(\mathbf{3 3 H}_{2}\right)=0.22\right)$. The solvent was removed in vacuo. The crude solid material was purified by preparative TLC (neutral alumina, BRocKmANn grade I, 50-200 $\mu \mathrm{m}, n$-hexane/THF/EtOAc 20:3:1, $\left.R_{\mathrm{f}}\left(\mathbf{3 3 H}_{2}\right)=0.40\right)$, followed by crystallization from a saturated solution of $\mathbf{3} 3 \mathbf{H}_{2}$ in $\mathrm{CH}_{2} \mathrm{Cl}_{2}$ layered with $\mathrm{MeOH}$. The product was obtained as dark green crystals $(8.0 \mathrm{mg}, 8 \%)$.

TLC:

$R_{\mathrm{f}}=0.22$ (n-hexane/THF/EtOAc 20:3:1); 
${ }^{1} \mathrm{H}$ NMR $\left(500 \mathrm{MHz}, \mathrm{CD}_{2} \mathrm{Cl}_{2}, 243 \mathrm{~K}\right) \quad \delta(\mathrm{ppm})=15.22(\mathrm{~s}, 2 \mathrm{H}, \mathrm{NpyH}), 7.98(\mathrm{~d}, J=7.7 \mathrm{~Hz}, 2 \mathrm{H}$, $\left.\mathrm{CH}^{\text {indole }}\right), 7.80\left(\mathrm{sbr}, 2 \mathrm{H}, \mathrm{Ph}^{\mathrm{B}}\right), 7.63\left(\mathrm{dt}, J=7.6,1.6 \mathrm{~Hz}, 2 \mathrm{H}, \mathrm{Ph}^{\mathrm{A}}\right)$, $7.48\left(\mathrm{t}, J=6.8 \mathrm{~Hz}, 6 \mathrm{H}, \mathrm{Ph}^{\mathrm{B}}\right), 7.46\left(\mathrm{sbr}, 2 \mathrm{H}, \mathrm{Ph}^{\mathrm{B}}\right), 7.34-7.30$ $\left(\mathrm{m}, 2 \mathrm{H}, \mathrm{Ph}^{\mathrm{C}}\right), 7.28\left(\mathrm{tt}, J=7.6,1.2 \mathrm{~Hz}, 2 \mathrm{H}, \mathrm{Ph}^{\mathrm{C}}\right), 7.23(\mathrm{dt}, J=3.7$, $1.2 \mathrm{~Hz}, 4 \mathrm{H}, \mathrm{Ph}^{\mathrm{C}}$ ), 7.19 (tdd, $J=8.7,6.6,1.5 \mathrm{~Hz}, 2 \mathrm{H}, \mathrm{Ph}^{\mathrm{A}}$ ), $7.16-7.11\left(\mathrm{~m}, 4 \mathrm{H}, \mathrm{Ph}^{\mathrm{A}}\right), 7.08\left(\mathrm{t}, J=7.6 \mathrm{~Hz}, 2 \mathrm{H}, \mathrm{Ph}^{\mathrm{A}}\right), 7.06(\mathrm{t}$, $J=7.7 \mathrm{~Hz}, 2 \mathrm{H}, \mathrm{CH}^{\text {indole }}$ ), 7.01 (ddd, $J=7.3,1.4,1.4 \mathrm{~Hz}, 2 \mathrm{H}$, $\mathrm{Ph}^{\mathrm{C}}$ ), $6.87\left(\mathrm{~d}, J=7.7 \mathrm{~Hz}, 2 \mathrm{H}, \mathrm{CH}^{\text {indole }}\right), 6.81(\mathrm{t}, J=7.7 \mathrm{~Hz}, 2 \mathrm{H}$, $\left.\mathrm{CH}^{\text {Indole }}\right), 2.28\left(\mathrm{dq}, J=14.6,7.4 \mathrm{~Hz}, 2 \mathrm{H},{ }^{\mathrm{a}} \mathrm{CH}_{2}\right), 2.11(\mathrm{dq}$, $J=15.3,7.5 \mathrm{~Hz}, 2 \mathrm{H}, \mathrm{d} \mathrm{CH}_{2}$ ), 2.01 (dq, $J=14.6,7.4 \mathrm{~Hz}, 2 \mathrm{H}$, $\left.{ }^{\mathrm{a} C H} \mathrm{C}_{2}\right), 1.52\left(\mathrm{dq}, J=14.5,7.5 \mathrm{~Hz}, 2 \mathrm{H}, \mathrm{cCH}_{2}\right.$ ), 1.29 (dq, $J=15.3$, $7.5 \mathrm{~Hz}, 4 \mathrm{H}, \mathrm{d} \mathrm{CH}_{2}$ ), 1.29 (dq, J=14.5, $7.5 \mathrm{~Hz}, 4 \mathrm{H}, \mathrm{bCH}_{2}$ ), 1.09 (dq, $J=14.5,7.5 \mathrm{~Hz}, 2 \mathrm{H}, \mathrm{bCH}_{2}$ ), 0.95 (dt, $J=14.5,7.5 \mathrm{~Hz}, 2 \mathrm{H}$, $\left.{ }^{\mathrm{c}} \mathrm{CH}_{2}\right), 0.65\left(\mathrm{t}, J=7.4 \mathrm{~Hz}, 6 \mathrm{H},{ }^{\mathrm{a}} \mathrm{CH}_{3}\right), 0.48(\mathrm{t}, J=7.5 \mathrm{~Hz}, 12 \mathrm{H}$, $\left.{ }^{\mathrm{d}} \mathrm{CH}_{3}\right), 0.45\left(\mathrm{t}, J=7.5 \mathrm{~Hz}, 12 \mathrm{H}, \mathrm{c}^{\mathrm{C}} \mathrm{CH}_{3},{ }^{\mathrm{b}} \mathrm{CH}_{3}\right)$;

${ }^{13} \mathrm{C}$ NMR $\left(126 \mathrm{MHz}, \mathrm{CD}_{2} \mathrm{Cl}_{2}, 243 \mathrm{~K}\right) \quad \delta(\mathrm{ppm})=162.6\left(\mathrm{C}_{\mathrm{q}}\right), 156.1\left(\mathrm{C}_{\mathrm{q}}\right), 150.9\left(\mathrm{C}_{\mathrm{q}}\right), 150.7\left(\mathrm{C}_{\mathrm{q}}\right)$, $145.2\left(\mathrm{C}_{\mathrm{q}}\right), 144.3\left(\mathrm{C}_{\mathrm{q}}\right), 143.7\left(\mathrm{C}_{\mathrm{q}}\right), 143.7\left(\mathrm{C}_{\mathrm{q}}\right), 142.3\left(\mathrm{C}_{\mathrm{q}}\right)$, $140.1\left(\mathrm{C}_{\mathrm{q}}\right), 139.9\left(\mathrm{C}_{\mathrm{q}}\right), 137.1\left(\mathrm{C}_{\mathrm{q}}\right), 136.3\left(\mathrm{C}_{\mathrm{q}}\right), 135.1\left(\mathrm{C}_{\mathrm{q}}\right)$, $133.6\left(\mathrm{C}_{\mathrm{q}}\right), 132.8\left(\mathrm{C}_{\mathrm{q}}\right), 132.8(\mathrm{br}, \mathrm{CH}), 131.9(\mathrm{CH}), 131.9$ (CH), $130.8(\mathrm{br}, \mathrm{CH}), 129.5(\mathrm{CH}), 128.7(\mathrm{CH}), 128.4(\mathrm{br}, \mathrm{CH})$, $128.2(\mathrm{CH}), 128.1(\mathrm{CH}), 128.0(\mathrm{CH}), 128.0(\mathrm{br}, \mathrm{CH}), 127.7$ $(\mathrm{CH}), 127.5(\mathrm{CH}), 127.1(\mathrm{CH}), 126.4(\mathrm{CH}), 125.6(\mathrm{CH}), 125.2$ $(\mathrm{CH}), 120.4(\mathrm{CH}), 119.6\left(\mathrm{C}_{\mathrm{q}}\right), 113.1\left(\mathrm{C}_{\mathrm{q}}\right), 110.4(\mathrm{CH}), 104.9$ $\left(\mathrm{C}_{\mathrm{q}}\right), 18.7\left(\mathrm{CH}_{2}\right), 18.3\left(\mathrm{CH}_{2}\right), 18.1\left(\mathrm{CH}_{2}\right), 17.2\left(\mathrm{CH}_{2}\right), 15.8$ $\left(\mathrm{CH}_{3}\right), 15.4\left(\mathrm{CH}_{3}\right), 15.2\left(\mathrm{CH}_{3}\right), 14.8\left(\mathrm{CH}_{3}\right)$;

IR (ATR, $\left.\mathrm{cm}^{-1}\right)$ :

$\tilde{v}=3052(\mathrm{vw}), 2962(\mathrm{w}), 2928(\mathrm{vw}), 2869(\mathrm{vw}), 1596(\mathrm{vw})$, 1542 (w), 1489 (w), 1459 (w), 1442 (w), 1399 (vw), 1360 (w), $1334(\mathrm{w}), 1310(\mathrm{w}), 1258(\mathrm{~m}), 1234(\mathrm{w}), 1174(\mathrm{vw})$, $1091(\mathrm{~m}), 1050$ (m), 1009 (s), 939 (vw), 917 (vw), $894(\mathrm{vw})$, 865 (w), 792 (vs), 767 (m), 734 (s), 696 (s);

$\mathrm{UV} /$ vis $\left(\mathrm{CH}_{2} \mathrm{Cl}_{2}\right)$ : $\lambda_{\max }\left(\mathrm{nm} ; \varepsilon\left[\mathrm{L} \mathrm{mol}^{-1} \mathrm{~cm}^{-1}\right]\right)=260$ (41600), 316 (37 600), 435 (34 900), 716 (28 000);

MS (ESI $\left.{ }^{+}, \mathrm{MeOH}\right)$ :

$m / z \quad(\%)=1297.7(100) \quad\left[33 \mathbf{H}_{2}+\mathrm{H}\right]^{+}, 1319.6 \quad(25)$ $\left[33 \mathrm{H}_{2}+\mathrm{Na}\right]^{+}$;

HR-MS (ESI $\left.{ }^{+}, \mathrm{MeOH}\right)$ : $m / z(\%)=1297.6564$ (calc. 1297.6579 for $\mathrm{C}_{92} \mathrm{H}_{81} \mathrm{~N}_{8}{ }^{+}$), 1319.6396 (calc. 1319.6398 for $\mathrm{C}_{92} \mathrm{H}_{80} \mathrm{~N}_{8} \mathrm{Na}^{+}$). 


\subsubsection{Nickel Complexes 35HNi and 34Ni}
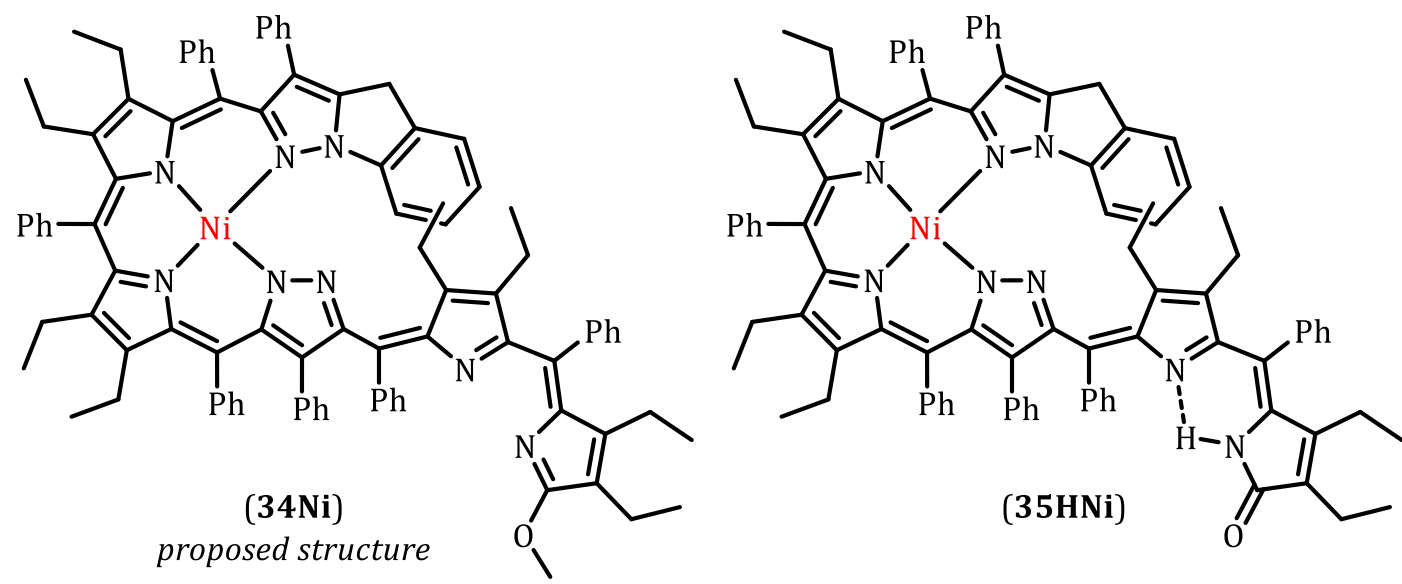

To a solution of mono-fused Siamese-twin porphyrin $\mathbf{3 2 H}_{3}(20 \mathrm{mg}, 15.4 \mu \mathrm{mol}, 1 \mathrm{eq})$ in a mixture of $\mathrm{MeOH} / \mathrm{CH}_{2} \mathrm{Cl}_{2}$ (each $3 \mathrm{~mL}$ ), $\mathrm{Ni}(\mathrm{OAc})_{2} \cdot 4 \mathrm{H}_{2} \mathrm{O}(115 \mathrm{mg}, 0.462 \mathrm{mmol}, 30 \mathrm{eq}$ ) was added all at once, and the mixture was stirred at r.t. for $48 \mathrm{~h}$. The reaction progress was monitored by successive mass spectrometry $(m / z=1299.7(\downarrow)$ and $1387.8(\uparrow))$. The crude solid material was purified by column chromatography (neutral alumina, BROCKMANN grade I, 50-200 $\mu \mathrm{m}, n$-hexane/EtOAc 6:1 $\rightarrow$ EtOAc, $\left.R_{\mathrm{f}}(\mathbf{3 4 N i})=0.40, R_{\mathrm{f}}(\mathbf{3 5 H N i})=0.00\right)$. The first $(34 \mathrm{Ni})$ and second (35HNi) yellow-green fractions were collected and the solvent was removed in vacuo (yield for $34 \mathrm{Ni}$ : $16.5 \mathrm{mg}, 77 \%$ ). Subsequent crystallization from a saturated solution of $35 \mathrm{HNi}$ in $\mathrm{CH}_{2} \mathrm{Cl}_{2}$ layered with $n$-pentane yielded dark yellow-green crystals (1.4 mg, $6 \%$ ).

\section{$34 \mathrm{Ni}$}

IR (ATR):

$\tilde{v}\left(\mathrm{~cm}^{-1}\right)=3055(\mathrm{vw}), 2960(\mathrm{w}), 2924(\mathrm{w}), 2868(\mathrm{w}), 1718$ (vw), $1700(\mathrm{vw}), 1684(\mathrm{vw}), 1675(\mathrm{vw}), 1597(\mathrm{vw}), 1559$ (vw), 1541 (vw), 1489 (vw), 1458 (m), 1429 (s), 1373 (w), 1350 (w), 1317 (vw), 1297 (vw), 1259 (s), 1174 (w), 1143 (w), $1094(\mathrm{~m}), 1051(\mathrm{~m}), 1018(\mathrm{~s}), 946(\mathrm{vw}), 929(\mathrm{w}), 865$ (w), 799 (s), 768 (vw), 745 (s), 693 (vs), 669 (w);

$\mathrm{UV} /$ vis $\left(\mathrm{CH}_{2} \mathrm{Cl}_{2}\right)$ : $\lambda_{\max }\left(\mathrm{nm} ; \varepsilon\left[\mathrm{L} \mathrm{mol}^{-1} \mathrm{~cm}^{-1}\right]\right)=311(20400), 406$ (24 700), 506 (6 100), 712 (6 000), 763 (3 800);

MS (ESI $\left.{ }^{+}, \mathrm{MeOH}\right)$ : $m / z(\%)=1387.6(100)[34 \mathrm{HNi}+\mathrm{H}]^{+} ;$

HR-MS (ESI $\left.{ }^{+}, \mathrm{MeOH}\right)$ : $m / z(\%)=1355.5933$ (calc. 1355.5932 for $\mathrm{C}_{93} \mathrm{H}_{85} \mathrm{~N}_{8} \mathrm{NiO}^{+}$).

\section{$35 \mathrm{HNi}$}

IR (ATR): 
(w), $1251(\mathrm{w}), 1234(\mathrm{w}), 1180(\mathrm{w}), 1112(\mathrm{~m}), 1071(\mathrm{w})$, $1050(\mathrm{~m}), 1118(\mathrm{~m}), 981(\mathrm{w}), 955(\mathrm{vw}), 931(\mathrm{w}), 905(\mathrm{w})$, $870(\mathrm{vw}), 840(\mathrm{~m}), 820(\mathrm{vw}), 796(\mathrm{vw}), 763(\mathrm{~m}), 741(\mathrm{~s}), 692$ (vs), $657(\mathrm{~m})$;

$\mathrm{UV} /$ vis $\left(\mathrm{CH}_{2} \mathrm{Cl}_{2}\right)$ :

$\lambda_{\max }\left(\mathrm{nm} ; \varepsilon\left[\mathrm{L} \mathrm{mol}^{-1} \mathrm{~cm}^{-1}\right]\right)=310$ (34 200), 407 (35 900), 465 (20 100), 673 (8 600), 724 (11 500);

$\mathrm{MS}\left(\mathrm{ESI}^{+}, \mathrm{MeOH}\right)$ :

$m / z(\%)=1373.6(100)[35 \mathbf{H N i}+\mathrm{H}]^{+}$;

HR-MS (ESI $\left.{ }^{+}, \mathrm{MeOH}\right)$ :

$m / z(\%)=1373.6041$ (calc. 1373.6038 for $\mathrm{C}_{92} \mathrm{H}_{83} \mathrm{~N}_{8} \mathrm{NiO}^{+}$).

\subsubsection{Dimanganese Complex 27 Mnz ${ }^{\text {ac }}$}

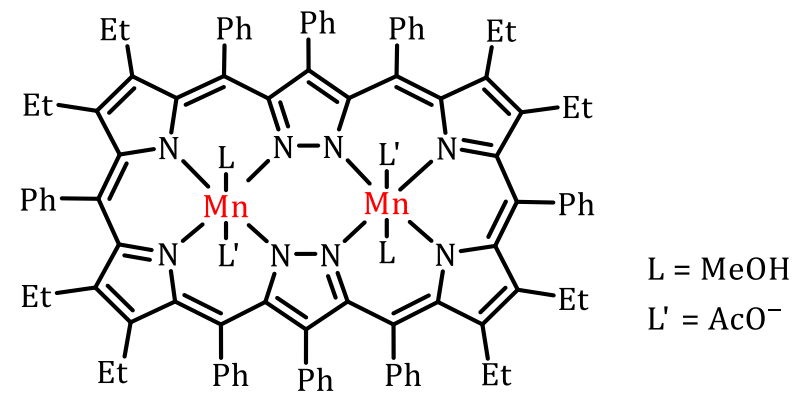

$\left(27 \mathrm{Mn}_{2}{ }^{\mathrm{ac}}\right)$

To a suspension of Siamese-twin porphyrin $\mathbf{2 7 \mathbf { H } _ { 4 }}(20 \mathrm{mg}, 15.4 \mu \mathrm{mol}, 1 \mathrm{eq})$ in anhydrous $\mathrm{MeOH}(20 \mathrm{~mL}), \mathrm{Mn}(\mathrm{OAc})_{2}(10.6 \mathrm{mg}, 61.3 \mu \mathrm{mol}, 4 \mathrm{eq})$ was added under inert conditions, and the mixture was stirred at room temperature for $48 \mathrm{~h}$ or for $30 \mathrm{~min}$ at $50^{\circ} \mathrm{C}$. The reaction progress was monitored by successive mass spectrometry $(\mathrm{m} / \mathrm{z}=1301.7(\downarrow), 1465.1(\uparrow)$ and $703.4(\uparrow)$ ). The solvent volume was reduced to a minimum $(<5 \mathrm{~mL})$ and the forming precipitate was filtered of and washed with cold $\mathrm{MeOH}$. The product was isolated as a darkgreen solid (10.0 mg, $40 \%$ ). Single crystals were obtained by slow evaporation of a saturated solution of $\mathbf{2 7} \mathbf{M n}_{2}$ ac in $\mathrm{MeOH}$.

IR (ATR):

$\tilde{v}\left(\mathrm{~cm}^{-1}\right)=2958(\mathrm{w}), 2925(\mathrm{w}), 2869(\mathrm{w}), 1623(\mathrm{w}), 1593$ (vw), 1563 (vw), 1492 (vw), 1466 (vw), 1444 (vw), 1421 (s), 1395 (m), 1373 (vw), 1358 (m), 1332 (vw), 1306 (m), 1279 (vw), 1261 (vw), 1242 (vw), 1220 (vw), $1175(\mathrm{w})$, $1156(\mathrm{w}), 1127$ (vw), $1108(\mathrm{~m}), 1074(\mathrm{vw}), 1056(\mathrm{~m}), 1015$ (m), $966(\mathrm{vw}), 929(\mathrm{w}), 892(\mathrm{~m}), 862(\mathrm{vw}), 843(\mathrm{vw}), 806$ (w), $776(\mathrm{~m}), 746$ (s), $694(\mathrm{vs}), 645$ (w), $604(\mathrm{vw}), 571(\mathrm{w})$, $541(\mathrm{w}), 492(\mathrm{~m}), 466(\mathrm{vw})$;

$\mathrm{UV} /$ vis $\left(\mathrm{CH}_{2} \mathrm{Cl}_{2}\right)$ : $\lambda_{\max }\left(\mathrm{nm} ; \varepsilon\left[\mathrm{L} \mathrm{mol}^{-1} \mathrm{~cm}^{-1}\right]\right)=296$ (55 000), 386 (62 800), 544 (17 100), 688 (14 600), 1015 (3 200);

$\mathrm{MS}\left(\mathrm{ESI}^{+}, \mathrm{MeOH}\right)$ :

$m / z(\%)=1466.5(100)\left[27 \mathbf{M n}_{2}{ }^{\mathrm{az}}\right]^{+} ;$

HR-MS (ESI $\left.{ }^{+}, \mathrm{MeOH}\right)$ : $m / z(\%)=1465.5388$ (calc. 1465.5394 for $\mathrm{C}_{94} \mathrm{H}_{83} \mathrm{~N}_{8} \mathrm{Mn}_{2} \mathrm{O}_{2}{ }^{+}$). 


\subsubsection{Dimanganese Complex 27Mnn $\mathbf{m}^{\mathrm{az}}$}

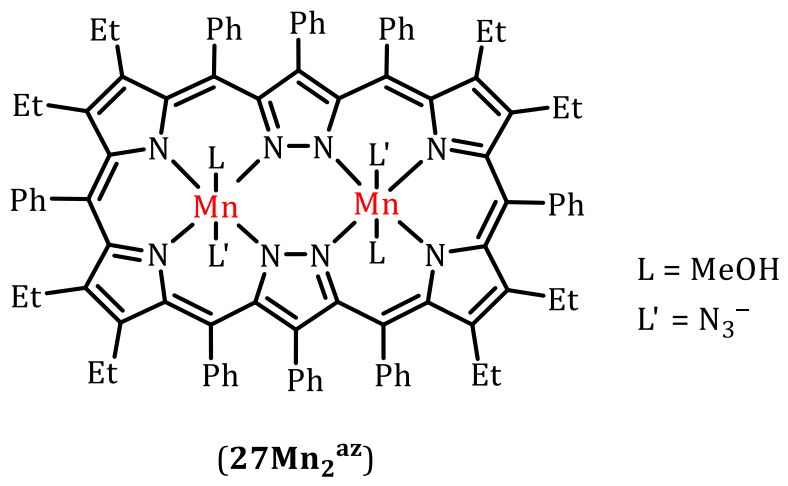

To a suspension of Siamese-twin porphyrin $\mathbf{2 7 \mathbf { H } _ { 4 }}(20 \mathrm{mg}, 15.4 \mu \mathrm{mol}, 1 \mathrm{eq})$ in anhydrous $\mathrm{MeOH}(20 \mathrm{~mL}), \mathrm{MnSO}_{4}(46.5 \mathrm{mg}, 0.31 \mathrm{mmol}, 20 \mathrm{eq}), \mathrm{NaN}_{3}(20.0 \mathrm{mg}, 0.31 \mathrm{mmol}, 20 \mathrm{eq})$ and $\mathrm{Na}_{2} \mathrm{CO}_{3}$ (33 mg, $0.31 \mathrm{mmol}, 20 \mathrm{eq}$ ) were added all at once under inert conditions, and the mixture was stirred at room temperature for $24 \mathrm{~h}$. The reaction progress was monitored by mass spectrometry $(\mathrm{m} / \mathrm{z}=1301.7(\downarrow), 1448.5(\uparrow)$ and $703.7(\uparrow))$. The mixture was filtered and the residue was washed with $\mathrm{MeOH}$ until the filtrate remained colorless. The crude product was dissolved in minimal amounts of $\mathrm{CH}_{2} \mathrm{Cl}_{2}$ or THF. Layering with $\mathrm{MeOH}$ yielded dark green crystals (12.0 mg, $50 \%$ ).

IR (ATR):

$\tilde{v}\left(\mathrm{~cm}^{-1}\right)=3059(\mathrm{vw}), 2964(\mathrm{w}), 2928(\mathrm{w}), 2870(\mathrm{w}), 2033$ (m, $\left.\mathrm{N}_{3}^{-}\right), 1596(\mathrm{vw}), 1560(\mathrm{w}), 1520(\mathrm{vw}), 1492(\mathrm{w}), 1466$ (vw), $1443(\mathrm{w}), 1417(\mathrm{~m}), 1372(\mathrm{vw}), 1312$ (vw), 1284 (vw), $1259(\mathrm{~m}), 1175(\mathrm{w}), 1159(\mathrm{vw}), 1092(\mathrm{~m}), 1073(\mathrm{~m})$, $1051(\mathrm{~m}), 1013(\mathrm{~s}), 929(\mathrm{w}), 892(\mathrm{w}), 861(\mathrm{vw}), 796(\mathrm{~s}), 775$ (s), 747 (s), 689 (vs);

$\mathrm{UV} /$ vis $\left(\mathrm{CH}_{2} \mathrm{Cl}_{2}\right)$ :

$\lambda_{\max }\left(\mathrm{nm} ; \varepsilon\left[\mathrm{L} \mathrm{mol}^{-1} \mathrm{~cm}^{-1}\right]\right)=295$ (46 900), 382 (52 400), 552 (13 400), 695 (13 200), 1018 (2 700);

MS (MALDI, DCTB-Matrix): $\quad m / z(\%)=1448.5(100)\left[\mathbf{2 7} \mathbf{M n}_{2}{ }^{\mathbf{a z}}\right]^{+}$;

HR-MS (MALDI, DCTB-Matrix): $\quad m / z(\%)=1448.5302$ (calc. 1448.5354 for $\mathrm{C}_{92} \mathrm{H}_{80} \mathrm{~N}_{11} \mathrm{Mn}_{2}{ }^{+}$). 
5.2.12 Nickel Manganese Complex 27NiMnac

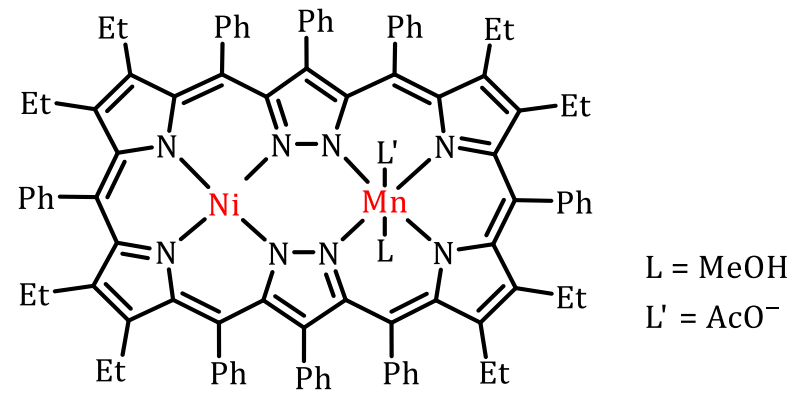

$\left(27 \mathrm{NiMn}^{\mathrm{ac}}\right)$

The mononickel complex of the Siamese-twin porphyrin $\mathbf{2 7} \mathbf{H}_{2} \mathbf{N i}$ was prepared according to literature. $[76,78,80]$ All analytical data agreed well with previous reports. To a solution of mononickel complex of the Siamese-twin porphyrin $\mathbf{2 7} \mathbf{H}_{\mathbf{4}}\left(\mathbf{2} \mathbf{2} \mathbf{H}_{2} \mathbf{N i}, 45 \mathrm{mg}, 33.1 \mu \mathrm{mol}, 1 \mathrm{eq}\right)$ in a mixture of anhydrous $\mathrm{CH}_{2} \mathrm{Cl}_{2} / \mathrm{MeOH}$ (each $30 \mathrm{~mL}$ ), $\mathrm{Mn}(\mathrm{OAc})_{2}$ (34.4 mg, $0.20 \mathrm{mmol}, 6 \mathrm{eq}$ ) was added all at once under inert conditions, and the mixture was stirred at room temperature overnight. The reaction progress was monitored by mass spectrometry $(m / z=1358.6(\downarrow)$ and $1410.5(\uparrow))$. The solvent was removed in vacuo. The crude solid material was purified by filtration through a short column (neutral alumina, BRocKMANN grade I, 50-200 $\mu \mathrm{m}$, EtOAc). The first brown fraction was collected and the solvent was removed in vacuo. The product $\mathbf{2 7} \mathbf{N i M n}{ }^{\text {ac }}$ was obtained as a dark brown powder $32.6 \mathrm{mg}$, $70 \%)$.

IR (ATR):

$\tilde{v}\left(\mathrm{~cm}^{-1}\right)=3054(\mathrm{vw}), 2963(\mathrm{w}), 2930(\mathrm{vw}), 2871(\mathrm{vw}), 1734$ (vw), 1685 (vw), 1597 (vw), 1450 (w), 1438 (w), $1419(\mathrm{w})$, 1375 (vw), 1350 (vw), 1258 (m), 1227 (vw), 1174 (vw), 1043 (m), 1013 (vs), 793 (s), 748 (m), 693 (s);

$\mathrm{UV} /$ vis $\left(\mathrm{CH}_{2} \mathrm{Cl}_{2}\right)$ : $\lambda_{\max }\left(\mathrm{nm} ; \varepsilon\left[\mathrm{L} \mathrm{mol}^{-1} \mathrm{~cm}^{-1}\right]\right)=288$ (46 200), 376 (64 700), 504 (21 600), 704 (18 300), 1199 (1 300);

$\mathrm{MS}\left(\mathrm{ESI}^{+}, \mathrm{MeOH}\right)$ :

$m / z(\%)=1410.5(100)\left[27 \mathrm{NiMn}^{\mathrm{ac}}\right]^{+} ;$

HR-MS (ESI $\left.{ }^{+}, \mathrm{MeOH}\right)$ : $m / z(\%)=1409.5225$ (calc. 1409.5234 for $\mathrm{C}_{92} \mathrm{H}_{80} \mathrm{~N}_{8} \mathrm{NiMn}^{+}$). 
5.2.13 Nickel Manganese Complex 27NiMnaz

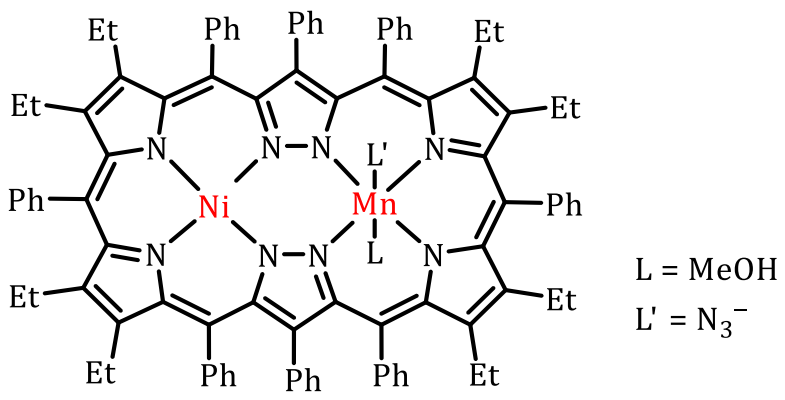

$\left(27 \mathrm{NiMn}^{\mathrm{az}}\right)$

The mononickel complex of the Siamese-twin porphyrin $\mathbf{2 7} \mathbf{H}_{2} \mathbf{N i}$ was prepared as described in the literature. ${ }^{[76,78,80]}$ All analytical data agreed well with previous reports. To a solution of mononickel complex of the Siamese-twin porphyrin $\mathbf{2 7}_{\mathbf{4}}\left(\mathbf{2} \mathbf{2} \mathbf{H}_{2} \mathbf{N i}, 20 \mathrm{mg}, 14.7 \mu \mathrm{mol}, 1 \mathrm{eq}\right)$ in a mixture of anhydrous $\mathrm{CH}_{2} \mathrm{Cl}_{2} / \mathrm{MeOH}$ (each $10 \mathrm{~mL}$ ), $\mathrm{MnSO}_{4}(23.1 \mathrm{mg}, 0.15 \mathrm{mmol}, 10 \mathrm{eq}$ ), $\mathrm{NaN}_{3}(10.0 \mathrm{mg}, 0.15 \mathrm{mmol}, 10 \mathrm{eq})$ and $\mathrm{Na}_{2} \mathrm{CO}_{3}(16.3 \mathrm{mg}, 0.15 \mathrm{mmol}, 10 \mathrm{eq})$ were added all at once under inert conditions, and the mixture was stirred at room temperature overnight. The reaction progress was monitored by TLC (silica, $n$-hexane/EtOAc 2:1, $R_{\mathrm{f}}=0.72$ ). The solvent was removed in vacuo. The crude solid material was purified by filtration through a short column (neutral alumina, BROCKMANN grade I, 50-200 $\mu \mathrm{m}, \mathrm{CH}_{2} \mathrm{Cl}_{2}$ ). The first brown fraction was collected and the solvent was removed in vacuo. The product $\mathbf{2 7} \mathbf{N i M n}^{\mathrm{az}}$ was obtained as a dark brown powder (18.3 mg, $84 \%$ ).

IR (ATR):

$\tilde{v}\left(\mathrm{~cm}^{-1}\right)=3055(\mathrm{vw}), 2963(\mathrm{w}), 2927(\mathrm{w}), 2870(\mathrm{w}), 1596$ (vw), 1560 (vw), 1490 (vm), 1437 (m), 1419 (m), 1374 (w), 1258 (s), 1175 (w), 1087 (m), 1045 (m), 1014 (vs), 929 (vw), 894 (vw), 863 (vw),794 (vs), 772 (m), 747 (m), 692 (vs), $664(\mathrm{~m})$;

$\mathrm{UV} /$ vis $\left(\mathrm{CH}_{2} \mathrm{Cl}_{2}\right)$ : $\lambda_{\max }\left(\mathrm{nm} ; \varepsilon\left[\mathrm{L} \mathrm{mol}^{-1} \mathrm{~cm}^{-1}\right]\right)=277$ (29 200), 382 (39 700), 530 (12 800), 709 (10 500), 1211 (2 400);

$\mathrm{MS}\left(\mathrm{ESI}^{+}, \mathrm{MeOH}\right)$ : $m / z(\%)=1410.5(100)\left[27 \mathrm{NiMn}^{\mathrm{az}}\right]^{+} ;$

HR-MS (ESI $\left.{ }^{+}, \mathrm{MeOH}\right)$ : $m / z(\%)=1409.5224$ (calc. 1409.5234 for $\mathrm{C}_{92} \mathrm{H}_{80} \mathrm{~N}_{8} \mathrm{NiMn}^{+}$). 


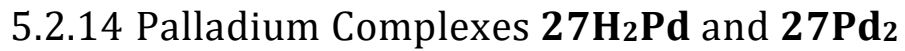

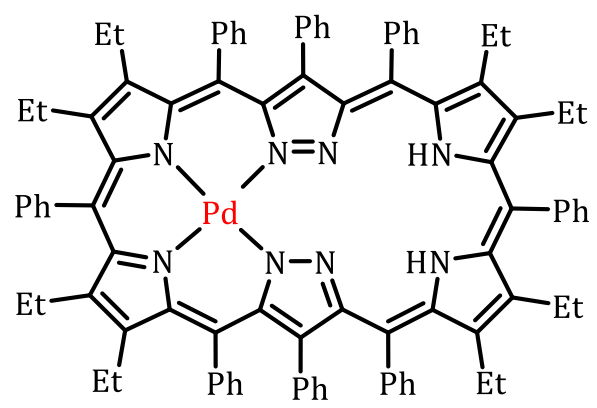

$\left(27 \mathrm{H}_{2} \mathrm{Pd}\right)$

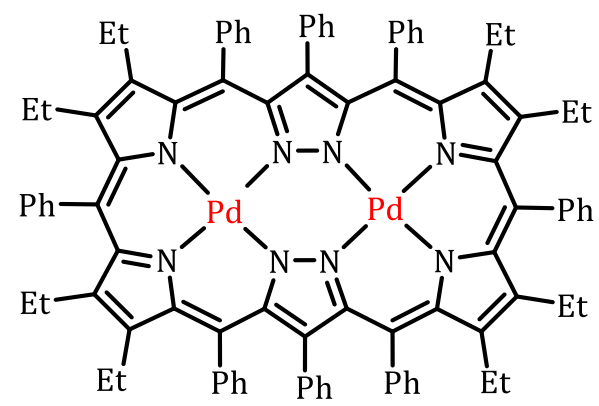

$\left(27 \mathrm{Pd}_{2}\right)$

To a suspension of Siamese-twin porphyrin $\mathbf{2 7} \mathbf{H}_{4}(100 \mathrm{mg}, 76.8 \mu \mathrm{mol}, 1 \mathrm{eq})$ in anhydrous $\mathrm{MeOH}(40 \mathrm{~mL}),\left[\mathrm{Pd}(\mathrm{acac})_{2}\right](140 \mathrm{mg}, 0.461 \mathrm{mmol}, 6 \mathrm{eq})$ was added all at once under inert conditions, and the mixture was heated to reflux for $48 \mathrm{~h}$. The reaction progress was monitored by TLC (neutral alumina, $n$-hexane/THF/EtOAc 20:3:1, $R_{\mathrm{f}}\left(\mathbf{2 7}_{\mathbf{4}}\right)=0.53$, $R_{\mathrm{f}}\left(\mathbf{2 7} \mathbf{H}_{2} \mathbf{P d}\right)=0.40$ ). The mixture was filtered and the residue was washed with $\mathrm{MeOH}$ until the filtrate remained colorless. The crude solid material was purified by column chromatography (neutral alumina, BRockMANN grade I, 50-200 $\mu \mathrm{m}, n$-hexane/THF/EtOAc 20:3:1, $\left.R_{\mathrm{f}}\left(\mathbf{2 7} \mathbf{H}_{\mathbf{2}} \mathbf{P d}\right)=0.40\right)$. The solvent was evaporated in vacuo. Subsequent crystallization from a saturated solution of $\mathbf{2} \mathbf{7 H}_{\mathbf{2}} \mathbf{P d}$ in THF layered with $\mathrm{MeOH}$ yielded dark green crystals ( $48.5 \mathrm{mg}, 43 \%$ ). After collection of crystalline material, the mother liquor was evaporated and the residue was redissolved in minimal amounts of $\mathrm{CH}_{2} \mathrm{Cl}_{2}$. Subsequent layering with $\mathrm{MeOH}$ yielded dark green crystals of $\mathbf{2 7} \mathbf{P d}_{2}(0.8 \mathrm{mg}, 1 \%)$.

\section{$27 \mathrm{H}_{2} \mathrm{Pd}$}

TLC:

$R_{\mathrm{f}}=0.40$ (n-hexane/THF/EtOAc 20:3:1);

${ }^{1} \mathrm{H}$ NMR $\left(500 \mathrm{MHz}, \mathrm{CD}_{2} \mathrm{Cl}_{2}, 243 \mathrm{~K}\right): \quad \delta(\mathrm{ppm})=9.77(\mathrm{sbr}, 1 \mathrm{H}, \mathrm{NH}), 7.57\left(\mathrm{~d}, J=6.3 \mathrm{~Hz}, 1 \mathrm{H}, \mathrm{Ph}^{\mathrm{D}}\right)$, $7.47-7.44\left(\mathrm{~m}, 1 \mathrm{H}, \mathrm{Ph}^{\mathrm{D}}\right), 7.44-7.35\left(\mathrm{~m}, 3 \mathrm{H}, \mathrm{Ph}^{\mathrm{D}}\right), 7.17$ (dd, $\left.J=7.3,2.2 \mathrm{~Hz}, 1 \mathrm{H}, \mathrm{Ph}^{\mathrm{C}}\right), 7.02(\mathrm{ddd}, J=7.6,4.0,2.1 \mathrm{~Hz}, 2 \mathrm{H}$, $\left.\mathrm{Ph}^{\mathrm{C}}\right), 6.98\left(\mathrm{t}, J=7.1 \mathrm{~Hz}, 1 \mathrm{H}, \mathrm{Ph}^{\mathrm{B}}\right), 6.92(\mathrm{tt}, J=7.2,1.4 \mathrm{~Hz}, 1 \mathrm{H}$, $\left.\mathrm{Ph}^{\mathrm{B}}\right), 6.85-6.75\left(\mathrm{~m}, 3 \mathrm{H}, \mathrm{Ph}^{\mathrm{C}}\right), 6.69-6.61\left(\mathrm{~m}, 3 \mathrm{H}, \mathrm{Ph}^{\mathrm{A}}, \mathrm{Ph}^{\mathrm{B}}\right)$, $6.60-6.53\left(\mathrm{~m}, 2 \mathrm{H}, \mathrm{Ph}^{\mathrm{A}}\right), 6.26\left(\mathrm{tt}, J=7.6,1.0 \mathrm{~Hz}, 1 \mathrm{H}, \mathrm{Ph}^{\mathrm{A}}\right)$, $5.91\left(\mathrm{dq}, J=7.6,1.2 \mathrm{~Hz}, 1 \mathrm{H}, \mathrm{Ph}^{\mathrm{A}}\right), 2.07(\mathrm{dq}, J=14.5,7.4 \mathrm{~Hz}$, $\left.1 \mathrm{H}, \mathrm{d}^{\mathrm{d}} \mathrm{CH}_{2}\right), 1.98\left(\mathrm{dq}, J=14.5,7.4 \mathrm{~Hz}, 2 \mathrm{H},{ }^{\mathrm{a}} \mathrm{CH}_{2},{ }^{\mathrm{b}} \mathrm{CH}_{2}\right), 1.89$ (dq, $\left.J=14.5,7.4 \mathrm{~Hz}, 2 \mathrm{H},{ }^{\mathrm{c}} \mathrm{CH}_{2}\right), 1.51\left(\mathrm{dt}, J=14.5,7.4 \mathrm{~Hz}, 1 \mathrm{H},{ }^{\mathrm{a}} \mathrm{CH}_{2}\right)$, 1.28 (dqd, $J=14.5,7.4,2.7 \mathrm{~Hz}, 2 \mathrm{H},{ }^{\mathrm{b}} \mathrm{CH}_{2},{ }^{\mathrm{d}} \mathrm{CH}_{2}$ ), $0.76(\mathrm{t}$, $\left.J=7.4 \mathrm{~Hz}, 3 \mathrm{H},{ }^{\mathrm{d}} \mathrm{CH}_{3}\right), 0.70\left(\mathrm{t}, J=7.4 \mathrm{~Hz}, 3 \mathrm{H},{ }^{\mathrm{c}} \mathrm{CH}_{3}\right), 0.64(\mathrm{t}$, $\left.J=7.4,3 \mathrm{H}, \mathrm{b}^{\mathrm{C}} \mathrm{CH}_{3}\right), 0.60\left(\mathrm{t}, J=7.4 \mathrm{~Hz}, 3 \mathrm{H}, \mathrm{aCH}_{3}\right)$; letters indicate correlated spin systems according to 2D NMR;

${ }^{13} \mathrm{C}$ NMR (126 MHz, $\left.\mathrm{CD}_{2} \mathrm{Cl}_{2}, 243 \mathrm{~K}\right): \delta(\mathrm{ppm})=158.2\left(\mathrm{C}_{\mathrm{q}}\right), 157.0\left(\mathrm{C}_{\mathrm{q}}\right), 153.3\left(\mathrm{C}_{\mathrm{q}}\right), 148.8(\mathrm{CH})$, $147.8\left(\mathrm{C}_{\mathrm{q}}\right), 147.1\left(\mathrm{C}_{\mathrm{q}}\right), 145.8\left(\mathrm{C}_{\mathrm{q}}\right), 144.3\left(\mathrm{C}_{\mathrm{q}}\right), 142.6(\mathrm{CH})$, $140.2\left(\mathrm{C}_{\mathrm{q}}\right), 138.9\left(\mathrm{C}_{\mathrm{q}}\right), 138.1(\mathrm{CH}), 137.7\left(\mathrm{C}_{\mathrm{q}}\right), 135.5\left(\mathrm{C}_{\mathrm{q}}\right)$, 
IR (ATR):

$134.8\left(\mathrm{C}_{\mathrm{q}}\right), 134.5\left(\mathrm{C}_{\mathrm{q}}\right), 134.4\left(\mathrm{C}_{\mathrm{q}}\right), 133.8(\mathrm{CH}), 133.1\left(\mathrm{C}_{\mathrm{q}}\right)$, $132.2\left(\mathrm{C}_{\mathrm{q}}\right), 132.0\left(\mathrm{C}_{\mathrm{q}}\right), 131.8(\mathrm{CH}), 131.6\left(\mathrm{C}_{\mathrm{q}}\right), 131.0(\mathrm{CH})$, $128.7(\mathrm{CH}), 128.6\left(\mathrm{C}_{\mathrm{q}}\right), 128.1(\mathrm{CH}), 128.0(\mathrm{CH}), 127.7(\mathrm{CH})$, $127.6(\mathrm{CH}), 127.4(\mathrm{CH}), 127.0(\mathrm{CH}), 126.7(\mathrm{CH}), 126.6(\mathrm{CH})$, $126.1(\mathrm{CH}), 124.5(\mathrm{CH}), 19.8\left(\mathrm{CH}_{2}\right), 19.2\left(\mathrm{CH}_{2}\right), 19.2\left(\mathrm{CH}_{2}\right)$, $18.8\left(\mathrm{CH}_{2}\right), 16.2\left(\mathrm{CH}_{3}\right), 16.1\left(\mathrm{CH}_{3}\right), 15.5\left(\mathrm{CH}_{3}\right), 15.4\left(\mathrm{CH}_{3}\right)$;

$\tilde{v}\left(\mathrm{~cm}^{-1}\right)=3054(\mathrm{vw}), 3021(\mathrm{vw}), 2968(\mathrm{w}), 2928(\mathrm{w}), 2869$ (w), 1595 (vw), 1577 (vw), 1532 (vw), 1473 (m), 1429 (s), 1372 (m), 1356 (m), 1315 (m), 1246 (w), 1189 (w), 1174 (w), $1153(\mathrm{w}), 1132(\mathrm{w}), 1113(\mathrm{~m}), 1054(\mathrm{~m}), 1013(\mathrm{~m}), 962$ (vw), $926(\mathrm{w}), 906(\mathrm{vw}), 891(\mathrm{~m}), 840(\mathrm{vw}), 811(\mathrm{vw}), 746$ $(\mathrm{s})$;

$\mathrm{UV} /$ vis $\left(\mathrm{CH}_{2} \mathrm{Cl}_{2}\right)$ :

$\lambda_{\max }\left(\mathrm{nm} ; \varepsilon\left[\mathrm{L} \mathrm{mol}^{-1} \mathrm{~cm}^{-1}\right]\right)=300$ (33 300), 408 (60 800), 477 (24 400), 610 (18 000), 662 (57 200), 799 (13 500);

MS (MALDI, DCTB-Matrix): $\quad m / z(\%)=1404.6(100)\left[\mathbf{2 7 H}_{2} \mathbf{P d}\right]^{+}$;

HR-MS (MALDI, DCTB-Matrix): $\quad m / z(\%)=1405.5836$ (calc. 1405.5797 for $\mathrm{C}_{92} \mathrm{H}_{83} \mathrm{~N}_{8} \mathrm{Pd}^{+}$).

27Pd 2

HR-MS $\left(\mathrm{ESI}^{+}, \mathrm{MeOH}\right): \quad \quad \quad m / z(\%)=1508.4627$ (calc. 1508.4575 for $\mathrm{C}_{92} \mathrm{H}_{81} \mathrm{~N}_{8} \mathrm{Pd}_{2}{ }^{+}$).

\subsubsection{Monoprotonated Complex $2 \mathbf{2 H}_{3} \mathbf{P d}^{+}$}

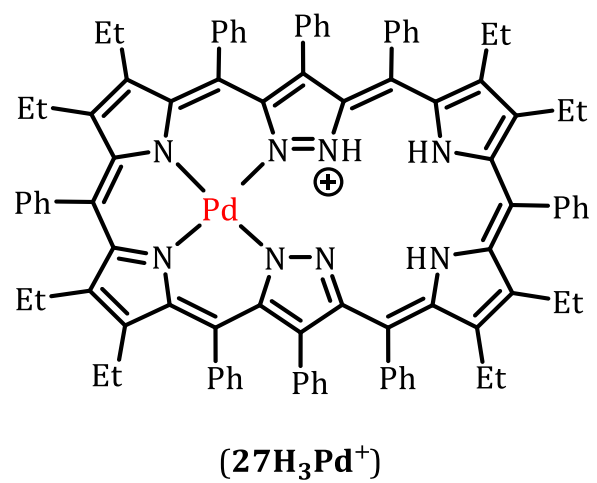

To a solution of Siamese-twin porphyrin complex $\mathbf{2 7 \mathbf { H } _ { 2 }} \mathbf{P d}(3 \mathrm{mg}, 2.1 \mu \mathrm{mol}, 1 \mathrm{eq})$ in $\mathrm{CD}_{2} \mathrm{Cl}_{2}$ $(400 \mu \mathrm{L})$, TFA ( $21 \mathrm{~mm}$ in $\left.\mathrm{CD}_{2} \mathrm{Cl}_{2}, 100 \mu \mathrm{L}, 2.1 \mu \mathrm{mol}, 1 \mathrm{eq}\right)$ was added all at once.

${ }^{1} \mathrm{H}$ NMR (500 MHz, $\left.\mathrm{CD}_{2} \mathrm{Cl}_{2}, 238 \mathrm{~K}\right) \quad \delta(\mathrm{ppm})=15.86(\mathrm{~s}, 1 \mathrm{H}, \mathrm{NH}), 14.18$ (s, 1H, NH), 8.83 (s, $1 \mathrm{H}, \mathrm{NH}), 7.59\left(\mathrm{~d}, J=6.3 \mathrm{~Hz}, 1 \mathrm{H}, \mathrm{Ph}^{\mathrm{D}}\right), 7.52(\mathrm{~d}, J=6.3 \mathrm{~Hz}, 2 \mathrm{H}$, $\left.\mathrm{Ph}^{\mathrm{D}}\right), 7.46\left(\mathrm{t}, J=6.3 \mathrm{~Hz}, 1 \mathrm{H}, \mathrm{Ph}^{\mathrm{D}}\right), 7.41\left(\mathrm{t}, J=6.3 \mathrm{~Hz}, 6 \mathrm{H}, \mathrm{Ph}^{\mathrm{D}}\right)$, $7.20-6.15(\mathrm{~m}, 29 \mathrm{H}, \mathrm{Ph}), 5.88-6.20(\mathrm{~d}, J=6.3 \mathrm{~Hz}, 1 \mathrm{H}, \mathrm{Ph})$, $0.76-0.69\left(\mathrm{~m}, 3 \mathrm{H}, \mathrm{CH}_{3}\right), 0.65-0.51\left(\mathrm{~m}, 9 \mathrm{H}, \mathrm{CH}_{3}\right)$; 
${ }^{13} \mathrm{C}$ NMR $\left(126 \mathrm{MHz}, \mathrm{CD}_{2} \mathrm{Cl}_{2}, 243 \mathrm{~K}\right) \quad \delta(\mathrm{ppm})=160.3\left(\mathrm{C}_{\mathrm{q}}\right), 160.0\left(\mathrm{C}_{\mathrm{q}}\right), 157.2\left(\mathrm{C}_{\mathrm{q}}\right), 152.5\left(\mathrm{C}_{\mathrm{q}}\right)$, $148.2\left(\mathrm{C}_{\mathrm{q}}\right), 147.7\left(\mathrm{C}_{\mathrm{q}}\right), 147.2\left(\mathrm{C}_{\mathrm{q}}\right), 145.6\left(\mathrm{C}_{\mathrm{q}}\right), 145.5\left(\mathrm{C}_{\mathrm{q}}\right)$, $143.5\left(\mathrm{C}_{\mathrm{q}}\right), 139.7\left(\mathrm{C}_{\mathrm{q}}\right), 137.7\left(\mathrm{C}_{\mathrm{q}}\right), 137.4\left(\mathrm{C}_{\mathrm{q}}\right), 136.7\left(\mathrm{C}_{\mathrm{q}}\right)$, $134.5(\mathrm{CH}), 133.8\left(\mathrm{C}_{\mathrm{q}}\right), 133.7\left(\mathrm{C}_{\mathrm{q}}\right), 132.1(\mathrm{CH}), 132.1(\mathrm{CH})$, $131.6(\mathrm{CH}), 131.0(\mathrm{CH}), 128.8(\mathrm{CH}), 128.7(\mathrm{CH}), 128.4(\mathrm{CH})$, $128.1(\mathrm{CH}), 128.0(\mathrm{CH}), 127.8(\mathrm{CH}), 127.1(\mathrm{CH}), 127.0(\mathrm{CH})$, $126.7(\mathrm{CH}), 126.5(\mathrm{CH}), 118.9\left(\mathrm{C}_{\mathrm{q}}\right), 116.6\left(\mathrm{C}_{\mathrm{q}}\right), 111.3(\mathrm{CH})$, $103.9(\mathrm{CH}), 19.9\left(\mathrm{CH}_{2}\right), 19.2\left(\mathrm{CH}_{2}\right), 19.2\left(\mathrm{CH}_{2}\right), 18.7\left(\mathrm{CH}_{2}\right)$, $16.1\left(\mathrm{CH}_{3}\right), 15.9\left(\mathrm{CH}_{3}\right), 15.1\left(\mathrm{CH}_{3}\right), 15.0\left(\mathrm{CH}_{3}\right)$;

$\mathrm{UV} /$ vis $\left(\mathrm{CH}_{2} \mathrm{Cl}_{2}\right)$ :

$\lambda_{\max }\left(\varepsilon\left[\mathrm{L} \mathrm{mol}^{-1} \mathrm{~cm}^{-1}\right]\right)=296$ (33 700), 398 (65 800), 469 (24 800), 589 (15 900), 644 (55 400), 767 (14 400) nm.

\subsubsection{Diprotonated Complex $\mathbf{2 7} \mathbf{H}_{4} \mathbf{P d}^{2+}$}

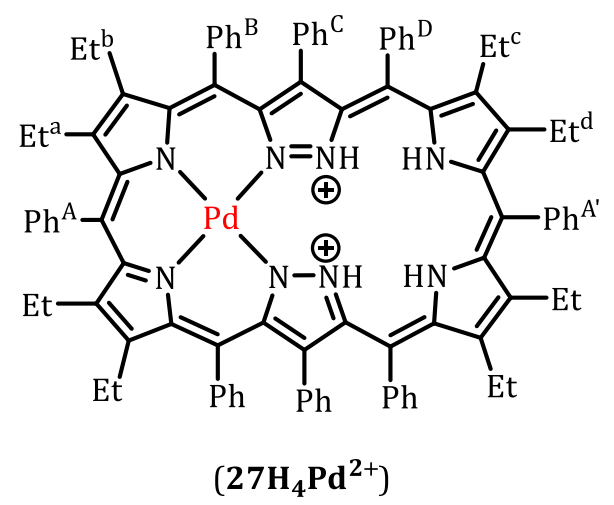

To a solution of Siamese-twin porphyrin complex $\mathbf{2 7 \mathbf { H } _ { 2 }} \mathbf{P d}\left(3 \mathrm{mg}, 2.1 \mu \mathrm{mol}, 1 \mathrm{eq}\right.$ ) in $\mathrm{CD}_{2} \mathrm{Cl}_{2}$ $(400 \mu \mathrm{L})$, TFA ( $21 \mathrm{~mm}$ in $\left.\mathrm{CD}_{2} \mathrm{Cl}_{2}, 200 \mu \mathrm{L}, 4.2 \mu \mathrm{mol}, 2 \mathrm{eq}\right)$ was added all at once.

${ }^{1} \mathrm{H}$ NMR $\left(500 \mathrm{MHz}, \mathrm{CD}_{2} \mathrm{Cl}_{2}, 238 \mathrm{~K}\right) \quad \delta(\mathrm{ppm})=16.25\left(\mathrm{~s}, 2 \mathrm{H}, \mathrm{N}{ }^{\mathrm{pz}} \mathrm{H}\right), 13.00(\mathrm{~s}, 2 \mathrm{H}, \mathrm{Npy}), 7.56$ (dd, $J=7.2,1.2 \mathrm{~Hz}, 2 \mathrm{H}$, ortho $\left.-\mathrm{Ph}^{\mathrm{A}}\right), 7.52(\mathrm{dd}, J=6.9,1.4 \mathrm{~Hz}$, $2 \mathrm{H}$ ortho- $\mathrm{Ph}^{\mathrm{A}^{\prime}}$ ), 7.47 (t, J = 7.3 Hz, 1H, para $-\mathrm{Ph}^{\mathrm{A}^{\prime}}$ ), $7.45-7.33$ (m, 5H, para $-\mathrm{Ph}^{\mathrm{A}^{\prime}}$, meta $-\mathrm{Ph}^{\mathrm{A}}$, meta $\left.-\mathrm{Ph}^{\mathrm{A}^{\prime}}\right), 7.09$ (d, $J=8.0 \mathrm{~Hz}$, $\left.2 \mathrm{H}, \mathrm{Ph}^{\mathrm{B}}\right), 7.05-6.94\left(\mathrm{~m}, 6 \mathrm{H}, \mathrm{Ph}^{\mathrm{C}}, \mathrm{Ph}^{\mathrm{D}}, \mathrm{Ph}^{\mathrm{D}}\right), 6.93(\mathrm{dt}, J=7.0$, $\left.2.1 \mathrm{~Hz}, 2 \mathrm{H}, \mathrm{Ph}^{\mathrm{B}}\right), 6.86\left(\mathrm{t}, J=7.4 \mathrm{~Hz}, 2 \mathrm{H}, \mathrm{Ph}^{\mathrm{C}}\right), 6.83-6.71(\mathrm{~m}$, $8 \mathrm{H}, \mathrm{Ph}^{\mathrm{A}}, \mathrm{Ph}^{\mathrm{C}}, \mathrm{Ph}^{\mathrm{C}}, \mathrm{Ph}^{\mathrm{C}}$ ), 6.67 (t, J=7.3 Hz, 2H, $\mathrm{Ph}^{\mathrm{B}}$ ), $6.64(\mathrm{td}$, $\left.J=7.2,2.0 \mathrm{~Hz}, 2 \mathrm{H}, \mathrm{Ph}^{\mathrm{D}}\right), 6.43\left(\mathrm{~d}, J=7.6,2 \mathrm{H}, \mathrm{Ph}^{\mathrm{D}}\right), 6.28(\mathrm{t}$, $\left.J=7.5 \mathrm{~Hz}, 2 \mathrm{H}, \mathrm{Ph}^{\mathrm{B}}\right), 5.82\left(\mathrm{~d}, J=7.6 \mathrm{~Hz}, 2 \mathrm{H}, \mathrm{Ph}^{\mathrm{B}}\right), 2.03(\mathrm{dt}$, $\left.J=14.0,6.9 \mathrm{~Hz}, 2 \mathrm{H},{ }^{\mathrm{a}} \mathrm{CH}_{2}\right), 1.95(\mathrm{dt}, J=14.6,7.3 \mathrm{~Hz}, 2 \mathrm{H}$, ${ }^{\mathrm{d}} \mathrm{CH}_{2}$ ), $1.82-1.65\left(\mathrm{~m}, 6 \mathrm{H},{ }^{\mathrm{b}} \mathrm{CH}_{2},{ }^{\mathrm{c}} \mathrm{CH}_{2}\right), 1.59(\mathrm{dq}, \mathrm{J}=14.6$, $7.4 \mathrm{~Hz}, 2 \mathrm{H}, \mathrm{b}_{2}$ ), 1.26 (dq, J=14.6, $7.3 \mathrm{~Hz}, 2 \mathrm{H},{ }^{\mathrm{d}} \mathrm{CH}_{2}$ ), 1.12 (dq, $\left.J=14.7,7.1 \mathrm{~Hz}, 2 \mathrm{H},{ }^{\mathrm{a}} \mathrm{CH}_{2}\right), 0.64\left(\mathrm{t}, J=7.4 \mathrm{~Hz}, 3 \mathrm{H},{ }^{\mathrm{d}} \mathrm{CH}_{3}\right.$ ), $0.58\left(\mathrm{t}, J=7.4 \mathrm{~Hz}, 3 \mathrm{H}, \mathrm{b}^{\mathrm{C}} \mathrm{CH}_{3}\right), 0.52\left(\mathrm{t}, J=7.8 \mathrm{~Hz}, 3 \mathrm{H}, \mathrm{a}^{\mathrm{a}} \mathrm{CH}_{3}\right)$, $0.50\left(\mathrm{t}, J=7.8 \mathrm{~Hz}, 3 \mathrm{H},{ }^{\mathrm{c}} \mathrm{CH}_{3}\right)$; 
${ }^{13} \mathrm{C}$ NMR $\left(126 \mathrm{MHz}, \mathrm{CD}_{2} \mathrm{Cl}_{2}, 298 \mathrm{~K}\right) \quad \delta(\mathrm{ppm})=160.8\left(\mathrm{C}_{\mathrm{q}}\right), 158.6\left(\mathrm{C}_{\mathrm{q}}\right), 149.5\left(\mathrm{C}_{\mathrm{q}}\right), 149.1\left(\mathrm{C}_{\mathrm{q}}\right)$, $147.3\left(\mathrm{C}_{\mathrm{q}}\right), 146.5\left(\mathrm{C}_{\mathrm{q}}\right), 146.1\left(\mathrm{C}_{\mathrm{q}}\right), 145.1\left(\mathrm{C}_{\mathrm{q}}\right), 145.1\left(\mathrm{C}_{\mathrm{q}}\right)$, $144.8\left(\mathrm{C}_{\mathrm{q}}\right), 138.8\left(\mathrm{C}_{\mathrm{q}}\right.$ axis $), 137.9\left(\mathrm{C}_{\mathrm{q}}\right), 137.5\left(\mathrm{C}_{\mathrm{q}}\right.$ axis $), 135.8\left(\mathrm{C}_{\mathrm{q}}\right)$, $134.5(\mathrm{CH}), 134.1(\mathrm{CH}), 134.1(\mathrm{CH}), 132.4(\mathrm{CH}), 132.1(\mathrm{CH})$, $132.1(\mathrm{CH}), 131.8(\mathrm{CH}), 131.7(\mathrm{CH}), 130.7(\mathrm{CH}), 129.0\left(\mathrm{C}_{\mathrm{q}}\right)$, $128.8(\mathrm{CH}), 128.7(\mathrm{CH}), 128.6(\mathrm{CH}), 128.4(\mathrm{CH}), 128.3(\mathrm{CH})$, $128.0(\mathrm{CH}), 128.0(\mathrm{CH}), 127.3(\mathrm{CH}), 127.1(\mathrm{CH}), 127.0(\mathrm{CH})$, $126.8(\mathrm{CH}), 126.0\left(\mathrm{C}_{\mathrm{q}}\right), 125.9(\mathrm{CH}), 124.0\left(\mathrm{C}_{\mathrm{q}}\right), 121.7\left(\mathrm{C}_{\mathrm{q}}\right)$, $118.7\left(\mathrm{C}_{\mathrm{q}}^{\text {axis }}\right), 116.4\left(\mathrm{C}_{\mathrm{q}}\right.$ axis $), 112.0\left(\mathrm{C}_{\mathrm{q}}\right), 105.1\left(\mathrm{C}_{\mathrm{q}}\right), 19.9$ $\left(\mathrm{CH}_{2}\right), 19.2\left(\mathrm{CH}_{2}\right), 19.0\left(\mathrm{CH}_{2}\right), 18.8\left(\mathrm{CH}_{2}\right), 16.0\left(\mathrm{CH}_{3}\right), 15.8$ $\left(\mathrm{CH}_{3}\right), 14.5\left(\mathrm{CH}_{3}\right), 14.5\left(\mathrm{CH}_{3}\right)$;

$\mathrm{UV} /$ vis $\left(\mathrm{CH}_{2} \mathrm{Cl}_{2}\right)$ : $\lambda_{\max }\left(\mathrm{nm} ; \varepsilon\left[\mathrm{L} \mathrm{mol}^{-1} \mathrm{~cm}^{-1}\right]\right)=296$ (34 100), 381 (75 500), 583 (17 800), 634 (59 400), 768 (20 100). 


\section{APPENDIX}

\subsection{X-ray Crystallographic Details}

\begin{tabular}{|c|c|c|c|}
\hline & $27 \mathrm{H}_{4}$ & $32 \mathrm{H}_{3}$ & $33 \mathrm{H}_{2}$ \\
\hline empirical formula & $\mathrm{C}_{104} \mathrm{H}_{96} \mathrm{~N}_{8}$ & $\mathrm{C}_{95} \mathrm{H}_{88} \mathrm{~N}_{8} \mathrm{O}$ & $\mathrm{C}_{93.50} \mathrm{H}_{83} \mathrm{Cl}_{3} \mathrm{~N}_{8}$ \\
\hline formula weight & 1457.88 & 1357.73 & 1425.02 \\
\hline crystal size $\left[\mathrm{mm}^{3}\right]$ & $0.38 \times 0.35 \times 0.12$ & $0.32 \times 0.18 \times 0.14$ & $0.32 \times 0.18 \times 0.15$ \\
\hline crystal system & triclinic & monoclinic & monoclinic \\
\hline space group & $P-1$ & $P 2_{1} / c$ & $P 22_{1} / n$ \\
\hline$a[\AA ̊]$ & $13.2177(6)$ & $25.9435(11)$ & $12.8191(4)$ \\
\hline$b[\AA]$ & $16.4528(7)$ & $11.0890(4)$ & $36.2081(13)$ \\
\hline$c[\AA]$ & $19.8772(9)$ & $28.1557(14)$ & $16.0472(5)$ \\
\hline$\alpha\left[^{\circ}\right]$ & $90.524(3)$ & 90 & 90 \\
\hline$\beta\left[^{\circ}\right]$ & $96.441(4)$ & $111.362(3)$ & $94.891(2)$ \\
\hline$\gamma\left[^{\circ}\right]$ & 109.183(3) & 90 & 90 \\
\hline$V\left[\AA^{3}\right]$ & $4052.0(3)$ & $7543.5(6)$ & $7421.3(4)$ \\
\hline$Z$ & 2 & 4 & 4 \\
\hline$\rho\left[\mathrm{g} / \mathrm{cm}^{3}\right]$ & 1.195 & 1.195 & 1.275 \\
\hline$F(000)$ & 1552 & 2888 & 3004 \\
\hline$\mu\left[\mathrm{mm}^{-1}\right]$ & 0.070 & 0.071 & 0.179 \\
\hline$T \min / T \max$ & 0.9843 / 0.9935 & 0.9837 / 0.9923 & 0.6464 / 0.8850 \\
\hline$\theta$-range $\left[{ }^{\circ}\right]$ & $1.312-25.692$ & $1.473-25.727$ & $1.392-25.611$ \\
\hline$h k l$-range & $\pm 16,-19-20,-4-23$ & $\pm 31,-13-12, \pm 34$ & $\pm 15, \pm 43, \pm 19$ \\
\hline measured refl. & 45374 & 68086 & 64622 \\
\hline unique refl. $[R$ int $]$ & $15244[0.1120]$ & $14263[0.1768]$ & $13954[0.1085]$ \\
\hline obs. refl. $(I>2 \sigma(I))$ & 9783 & 7305 & 9287 \\
\hline data / res. / param. & 15244 / 0 / 1082 & 14263 / 3 / 956 & 13954 / 104 / 996 \\
\hline goodness-of-fit $\left(\mathrm{F}^{2}\right)$ & 1.001 & 1.037 & 1.035 \\
\hline $\mathrm{R} 1, \mathrm{wR} 2(I>2 \sigma(I))$ & $0.0618,0.1149$ & $0.0924,0.1273$ & $0.0758,0.1884$ \\
\hline R1, wR2 (all data) & $0.1106,0.1304$ & $0.1834,0.1523$ & $0.1140,0.2113$ \\
\hline res. e. dens. $\left[\mathrm{e} / \AA^{3}\right]$ & $-0.241 / 0.248$ & $-0.186 / 0.232$ & $-0.893 / 1.138$ \\
\hline CCDC Number & 1424113 & 1424114 & 1424115 \\
\hline
\end{tabular}




\begin{tabular}{|c|c|c|c|}
\hline & $35 \mathrm{HNi}$ & 46 and 49 & $27 \mathrm{Mn}_{2}^{\mathrm{ac}} \times \mathrm{MeOH}$ \\
\hline empirical formula & $\mathrm{C}_{94} \mathrm{H}_{86} \mathrm{C}_{14} \mathrm{~N}_{8} \mathrm{NiO}$ & $\mathrm{C}_{33} \mathrm{H}_{30} \mathrm{~N}_{4} \mathrm{O}$ & $\mathrm{C}_{98} \mathrm{H}_{92} \mathrm{Mn}_{2} \mathrm{~N}_{8} \mathrm{O}_{6}$ \\
\hline formula weight & 1544.21 & 498.61 & 1587.68 \\
\hline crystal size $\left[\mathrm{mm}^{3}\right]$ & $0.50 \times 0.41 \times 0.18$ & $0.26 \times 0.09 \times 0.05$ & $0.15 \times 0.12 \times 0.11$ \\
\hline crystal system & triclinic & monoclinic & monoclinic \\
\hline space group & $P-1$ & $P 2_{1} / c$ & $C 2 / c$ \\
\hline$a[\AA ̊]$ & $15.8185(4)$ & $5.8557(4)$ & $19.1880(7)$ \\
\hline$b[\AA ̊]$ & $15.8608(5)$ & $26.5087(15)$ & $21.9945(7)$ \\
\hline$c[\AA]]$ & $19.2496(6)$ & $16.9030(10)$ & $23.7483(8)$ \\
\hline$\alpha\left[^{\circ}\right]$ & $85.417(2)$ & 90 & 90 \\
\hline$\beta\left[^{\circ}\right]$ & $78.652(2)$ & $93.639(2)$ & 109.353 \\
\hline$\gamma\left[{ }^{\circ}\right]$ & $78.874(2)$ & 90 & 90 \\
\hline$V\left[\AA^{3}\right]$ & $4641.8(2)$ & $2618.5(3)$ & $9456.2(6)$ \\
\hline$Z$ & 2 & 4 & 4 \\
\hline$\rho\left[\mathrm{g} / \mathrm{cm}^{3}\right]$ & 1.105 & 1.265 & 1.115 \\
\hline$F(000)$ & 1620 & 1056 & 3336 \\
\hline$\mu\left[\mathrm{mm}^{-1}\right]$ & 0.371 & 0.078 & 0.320 \\
\hline$T_{\min } / T_{\max }$ & 0.9255 / 0.8030 & $0.7438 / 0.7205$ & - \\
\hline$\theta$-range $\left[{ }^{\circ}\right]$ & $1.310-25.629$ & $2.415-28.348$ & $1.46-25.66$ \\
\hline hkl-range & $-16-19, \pm 19, \pm 23$ & $\pm 7, \pm 35, \pm 22$ & $\pm 23,-26-25,-28-27$ \\
\hline measured refl. & 54111 & 79263 & 39913 \\
\hline unique refl. $\left[R_{\text {int }}\right]$ & $17474[0.0355]$ & 6527 [0.1207] & 8933 [0.1219] \\
\hline obs. refl. $(I>2 \sigma(I))$ & 13391 & 4119 & 6125 \\
\hline data / res. / param. & 17474 / 9 / 995 & 6527 / 0 / 355 & 8933 / 142 / 576 \\
\hline goodness-of-fit $\left(F^{2}\right)$ & 1.015 & 1.044 & 1.055 \\
\hline$R 1, w R 2(I>2 \sigma(I))$ & $0.0442,0.1120$ & $0.0566,0.1040$ & $0.0784,0.1644$ \\
\hline$R 1, w R 2$ (all data) & $0.0610,0.1178$ & $0.1160,0.1232$ & $0.1204,0.1818$ \\
\hline res. e. dens. $\left[\mathrm{e} / \AA^{3}\right]$ & $-0.616 / 1.030$ & $-0.316 / 0.241$ & $-0.460 / 0.934$ \\
\hline CCDC Number & - & - & - \\
\hline
\end{tabular}




\begin{tabular}{|c|c|c|c|}
\hline $27 \mathrm{Mn}_{2}^{\mathrm{az}} \times \mathrm{MeOH}$ & $27 \mathrm{Mn}_{2}{ }^{\mathrm{az}} \times \mathrm{THF}$ & $27 \mathrm{H}_{2} \mathrm{Pd}$ & $27 \mathbf{P d}_{2}$ \\
\hline $\mathrm{C}_{95} \mathrm{H}_{92} \mathrm{Mn}_{2} \mathrm{~N}_{14} \mathrm{O}_{3}$ & $\mathrm{C}_{99} \mathrm{H}_{94} \mathrm{Mn}_{2} \mathrm{~N}_{14} \mathrm{O}_{2}$ & $\mathrm{C}_{106.20} \mathrm{H}_{116.70} \mathrm{~N}_{8} \mathrm{O}_{5.95} \mathrm{Pd}_{1}$ & $\mathrm{C}_{92} \mathrm{H}_{80} \mathrm{~N}_{8} \mathrm{Pd}_{2}$ \\
\hline 1587.70 & 1621.76 & 1712.09 & 1510.44 \\
\hline $0.50 \times 0.27 \times 0.25$ & $0.50 \times 0.38 \times 0.23$ & $0.33 \times 0.30 \times 0.10$ & $0.50 \times 0.34 \times 0.22$ \\
\hline monoclinic & triclinic & triclinic & monoclinic \\
\hline$C 2 / c$ & $P-1$ & $P-1$ & $C 2 / c$ \\
\hline $35.9287(12)$ & $14.5380(5)$ & $15.2345(4)$ & $35.2667(10)$ \\
\hline $11.4990(2)$ & $18.4615(6)$ & $16.9566(4)$ & $11.9903(5)$ \\
\hline $26.0141(9)$ & $18.7480(6)$ & $19.4273(5)$ & $25.8605(7)$ \\
\hline 90 & $75.667(2)$ & $101.572(2)$ & 90 \\
\hline $128.189(2)$ & $78.359(3)$ & $96.479(2)$ & $127.407(2)$ \\
\hline 90 & $84.598(3)$ & $111.128(2)$ & 90 \\
\hline $8447.3(5)$ & $4769.8(3)$ & $4490.9(2)$ & $8686.4(5)$ \\
\hline 4 & 2 & 2 & 4 \\
\hline 1.248 & 1.129 & 1.266 & 1.155 \\
\hline 3336 & 1704 & 1812 & 3120 \\
\hline 0.358 & 0.318 & 0.277 & 0.460 \\
\hline $0.9484 / 0.8328$ & $0.9373 / 0.8263$ & $0.8836 / 0.6963$ & $0.9226 / 0.7679$ \\
\hline $1.442-25.646$ & $1.411-25.742$ & $1.333-26.731$ & $1.454-25.699$ \\
\hline $\pm 43, \pm 13, \pm 31$ & $\pm 17, \pm 22, \pm 22$ & $-19-18, \pm 21, \pm 24$ & $\pm 42, \pm 14, \pm 31$ \\
\hline 48469 & 59782 & 63940 & 48565 \\
\hline 7947 [0.0587] & 18013 [0.0462] & $19030[0.0421]$ & $8211[0.0784]$ \\
\hline 7129 & 13637 & 15886 & 6490 \\
\hline 7947 / 11 / 573 & 18013 / 0 / 1080 & 19030 / 577 / 1281 & $8211 / 0$ / 464 \\
\hline 1.209 & 0.996 & 1.022 & 1.055 \\
\hline $0.0631,0.1582$ & $0.0472,0.1128$ & $0.0387,0.0929$ & $0.0626,0.1731$ \\
\hline $0.0705,0.1617$ & $0.0675,0.1203$ & $0.0517,0.0973$ & $0.0793,0.1836$ \\
\hline$-0.458 / 1.071$ & $-0.376 / 0.745$ & $-0.555 / 0.503$ & $-0.837 / 0.804$ \\
\hline- & - & - & - \\
\hline
\end{tabular}



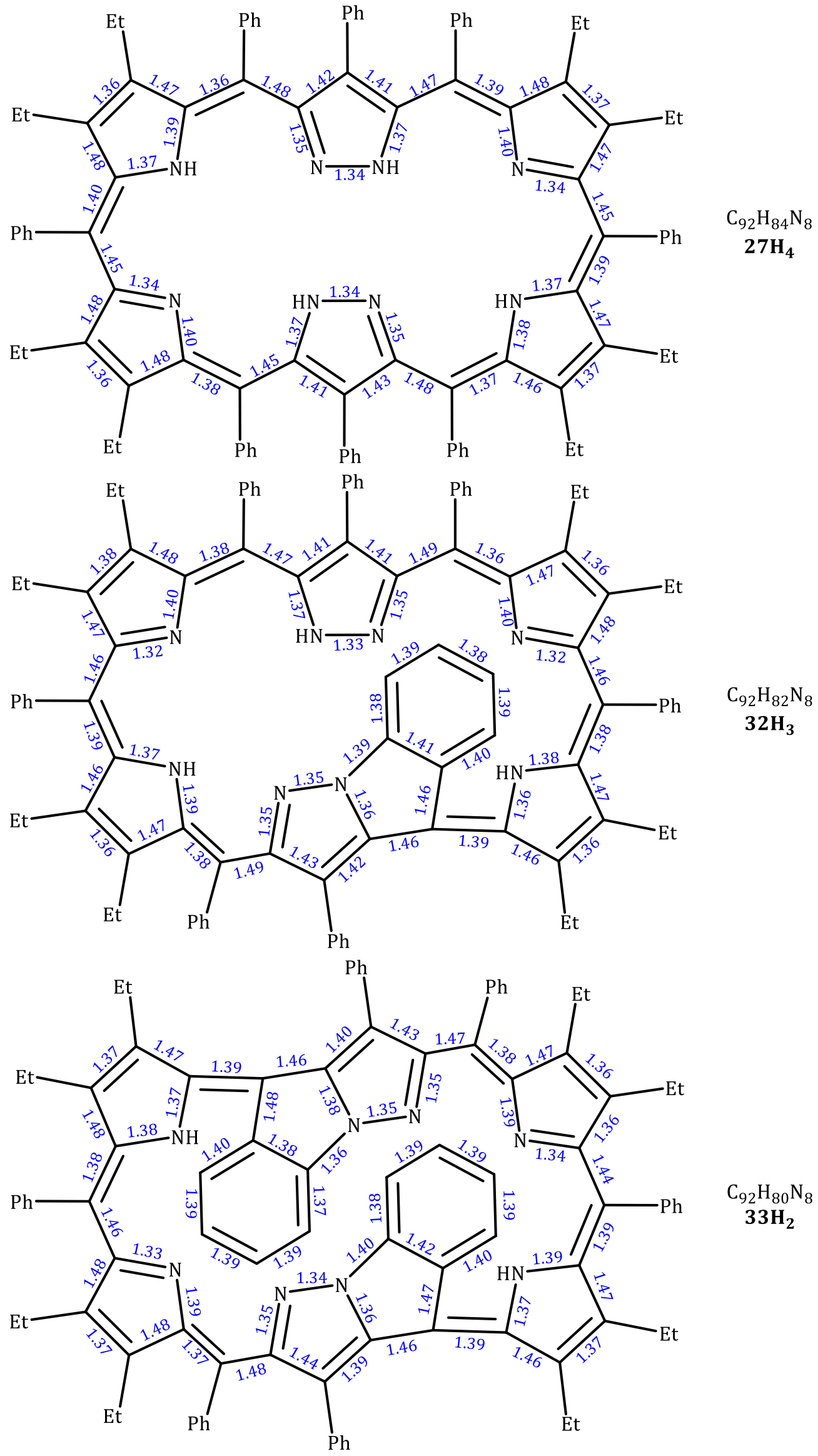

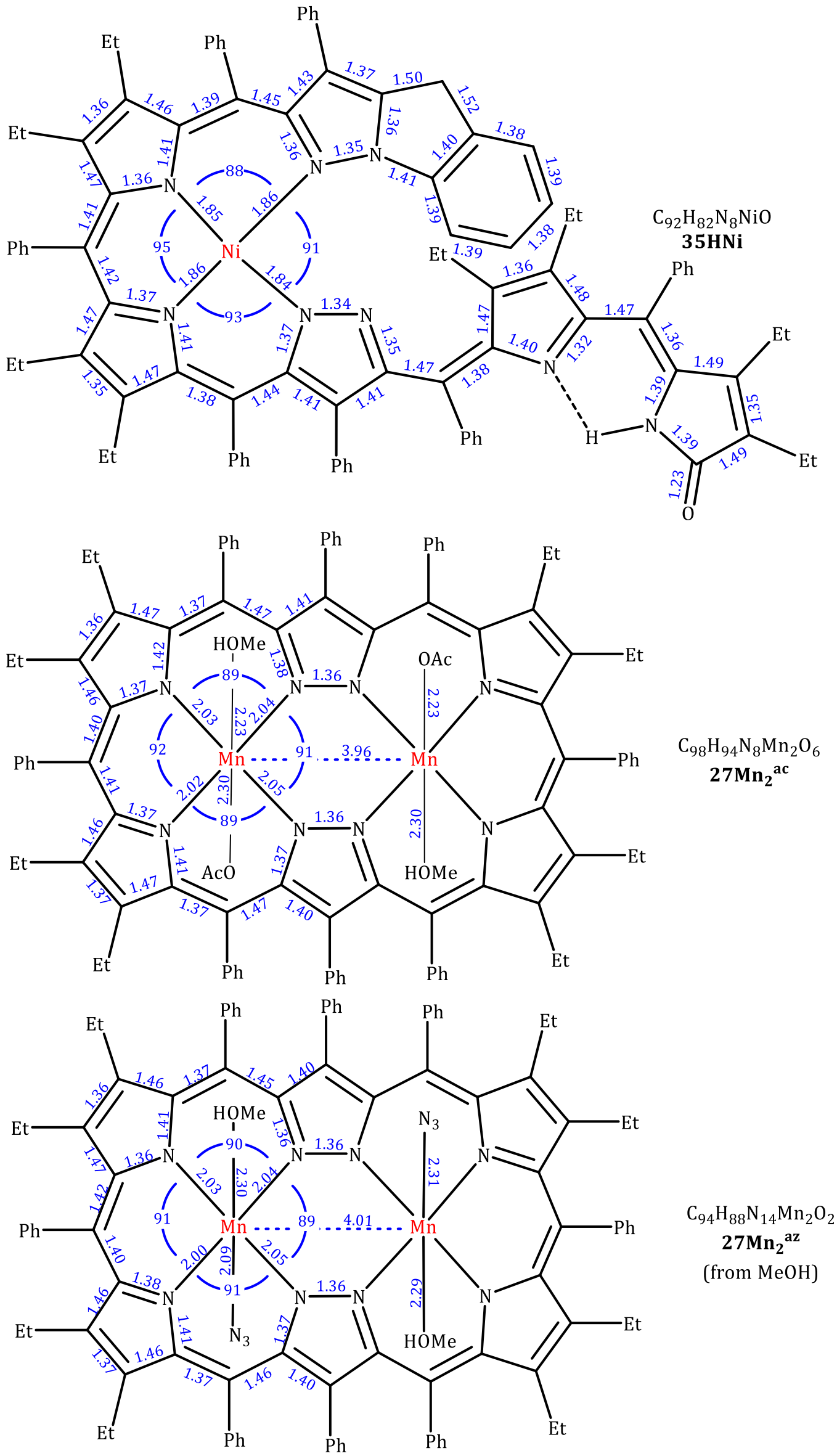

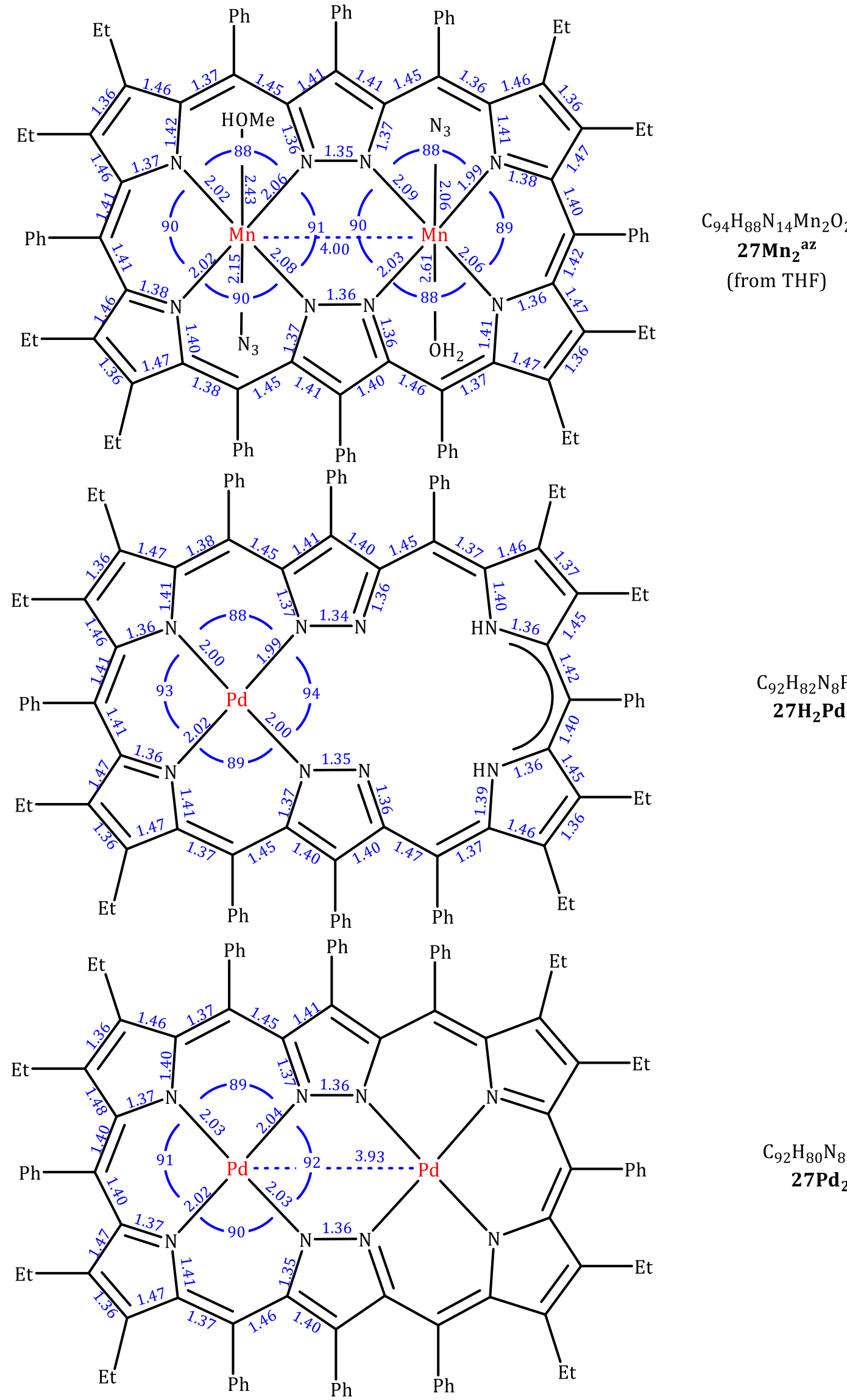

$\mathrm{C}_{92} \mathrm{H}_{80} \mathrm{~N}_{8} \mathrm{Pd}_{2}$ ${ }_{27} \mathbf{P d}_{2}$ 


\subsection{Spectroscopic Details}

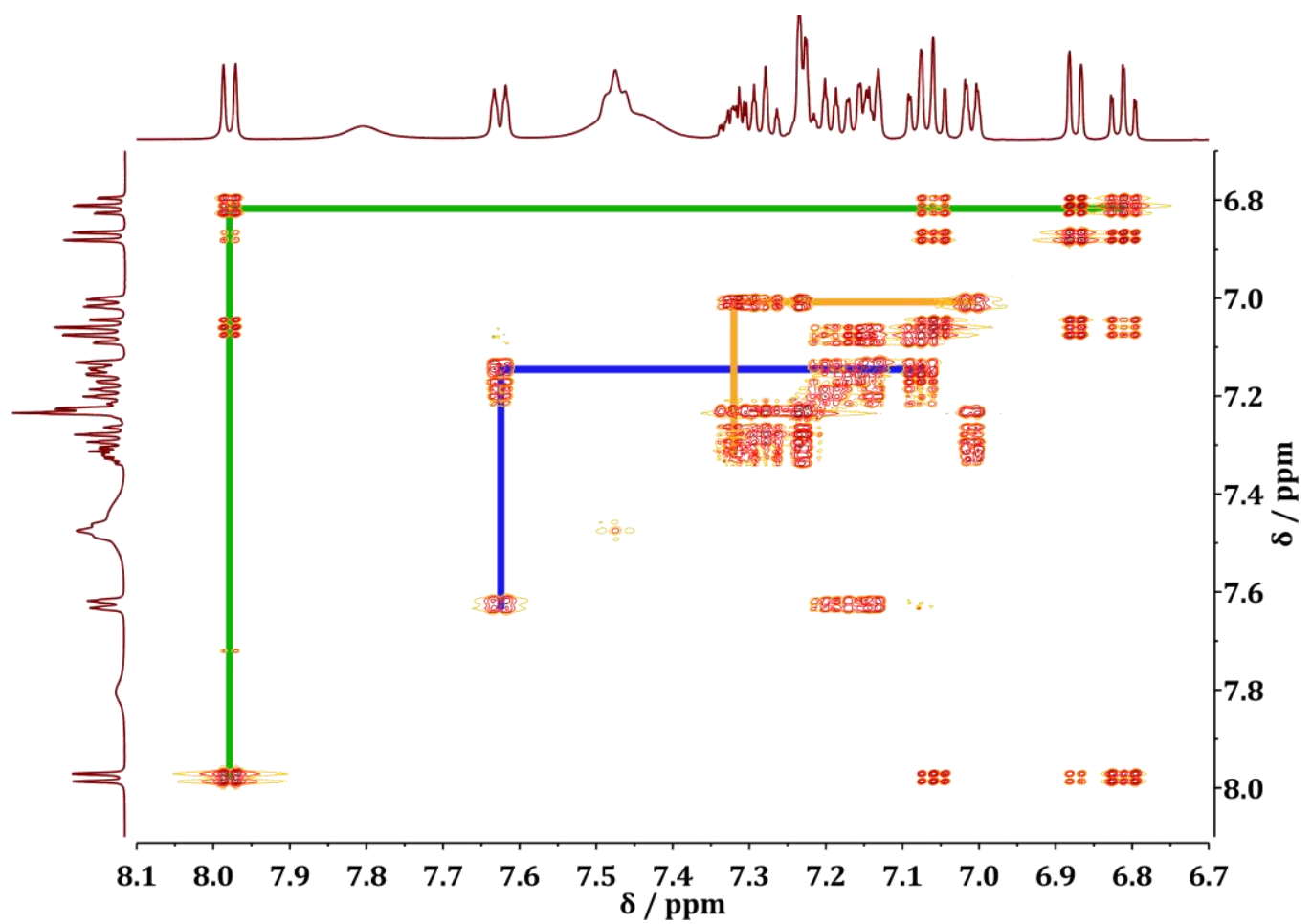

Figure S1: Section ( $\delta=8.1-6.7 \mathrm{ppm}$ ) of ${ }^{1} \mathrm{H}^{1} \mathrm{H}-\mathrm{COSY}$ spectrum $\left(500 \mathrm{MHz}, \mathrm{CD}_{2} \mathrm{Cl}_{2}, 298 \mathrm{~K}\right.$ ) of bis-fused Siamese-twin porphyrin $\mathbf{3 3} \mathbf{H}_{2}$. Correlating spin systems are connected by colored lines.

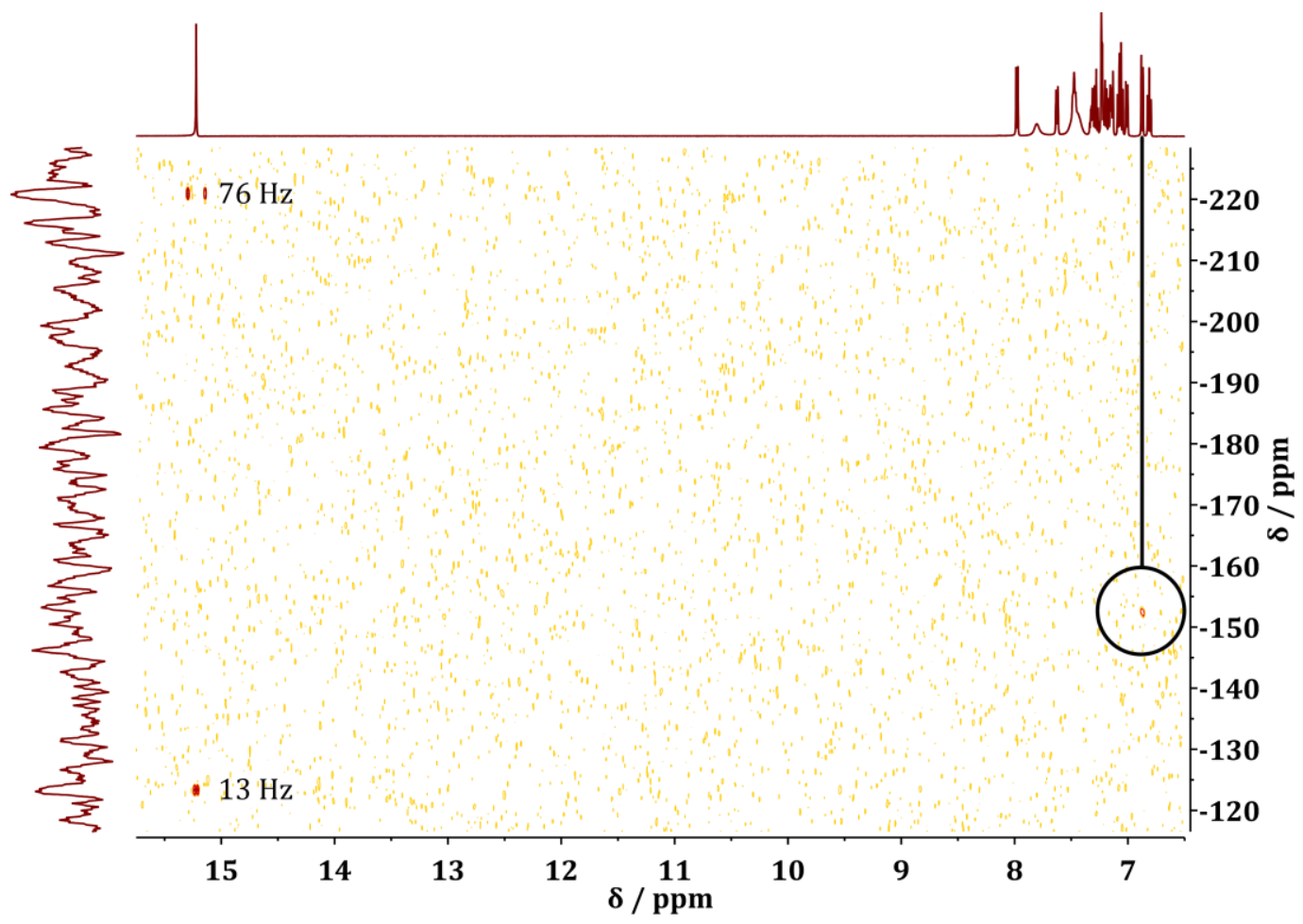

Figure S2: ${ }^{1} \mathrm{H}^{15} \mathrm{~N}$ HMBC spectrum $\left(500 \mathrm{MHz}, \mathrm{CD}_{2} \mathrm{Cl}_{2}, 243 \mathrm{~K}\right)$ of $\mathbf{3 3 H}_{2}$. Coupling constants of ${ }^{1} \mathrm{~J}(\mathrm{NH})=76 \mathrm{~Hz}$ and $1 \mathrm{~h}(\mathrm{NH})=13 \mathrm{~Hz}$ suggest the presence of strong hydrogen bonding within the dipyrrin unit. The phenyl-to-pyrazole fusion is evidenced by correlation between a phenyl proton and a pyrazole nitrogen atom ( $\delta=6.87$ and $152 \mathrm{ppm})$, encircled in black. 


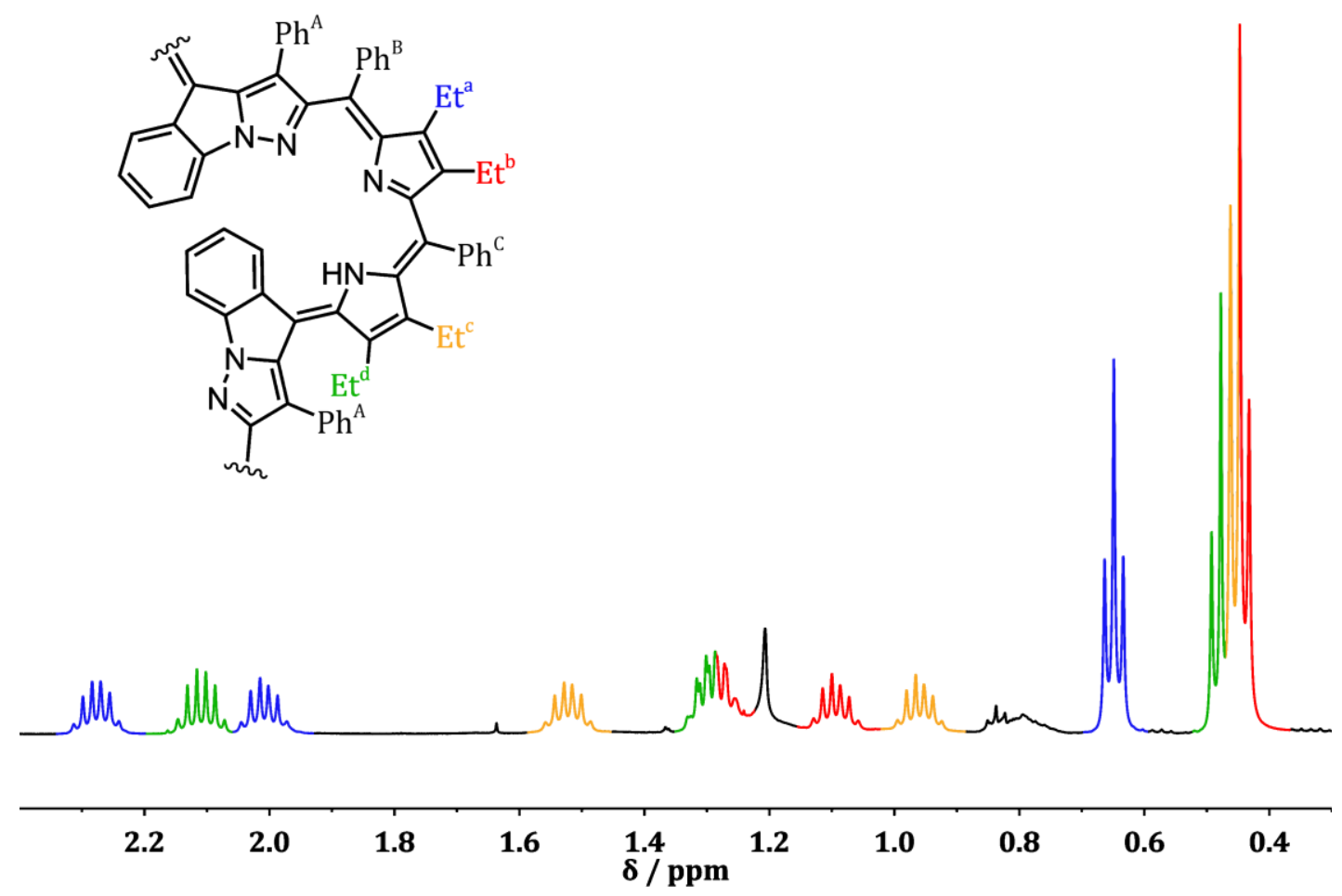

Figure S3: Section $(\delta=2.4-0.3 \mathrm{ppm})$ of ${ }^{1} \mathrm{H}$ NMR spectrum $\left(500 \mathrm{MHz}, \mathrm{CD}_{2} \mathrm{Cl}_{2}, 243 \mathrm{~K}\right)$ of $\mathbf{3 3} \mathbf{H}_{2}$. Correlated spin systems are colored equally according to the ${ }^{1} \mathrm{H}^{1} \mathrm{H}$ COSY shown below.

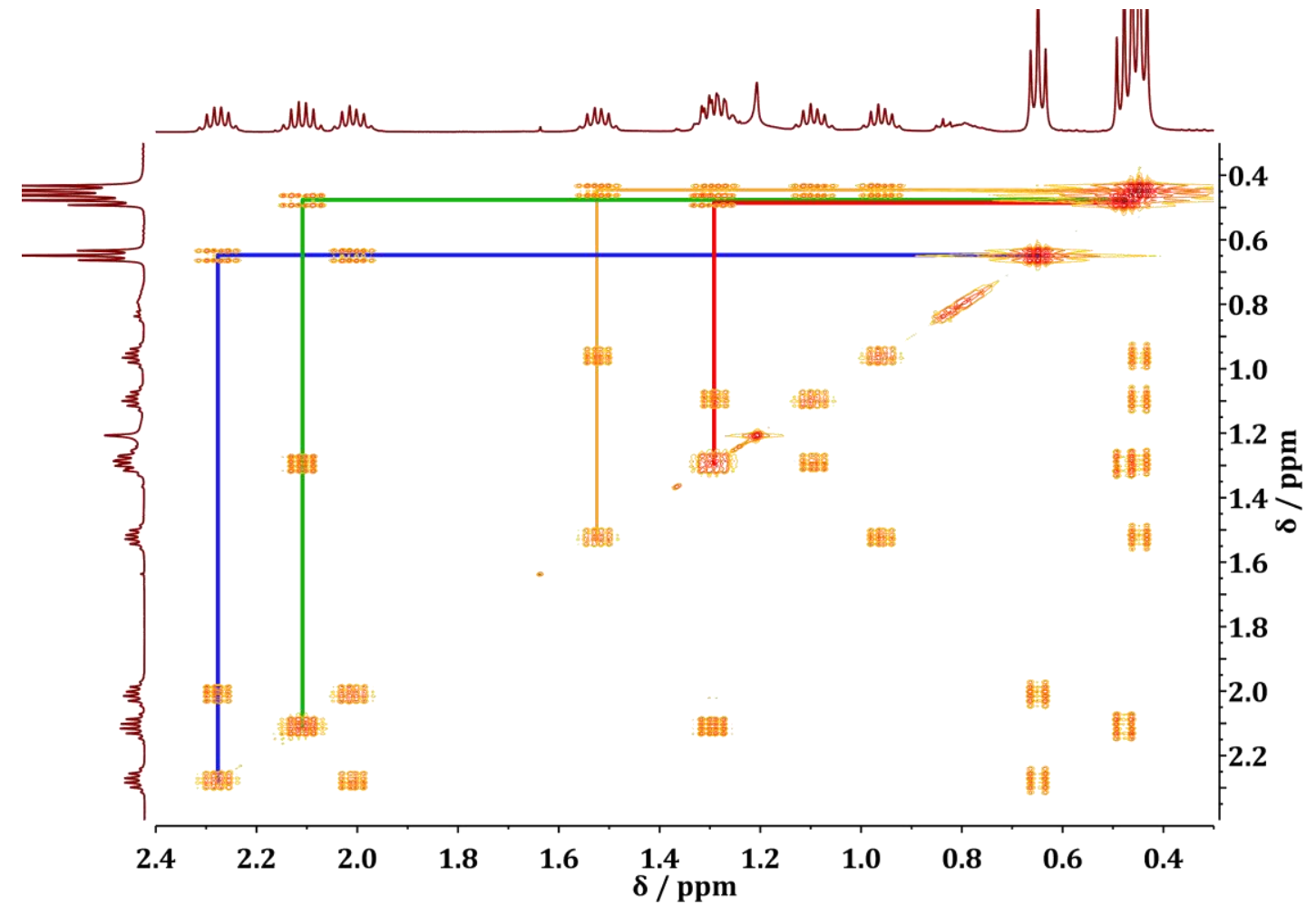

Figure S4: Section ( $\delta=2.4-0.3 \mathrm{ppm}$ ) of ${ }^{1} \mathrm{H}^{1} \mathrm{H}-\mathrm{COSY}$ spectrum $\left(500 \mathrm{MHz}, \mathrm{CD}_{2} \mathrm{Cl}_{2}, 298 \mathrm{~K}\right.$ ) of bis-fused Siamese-twin porphyrin $\mathbf{3 3} \mathbf{H}_{2}$. Correlated spin systems are connected by colored lines. 


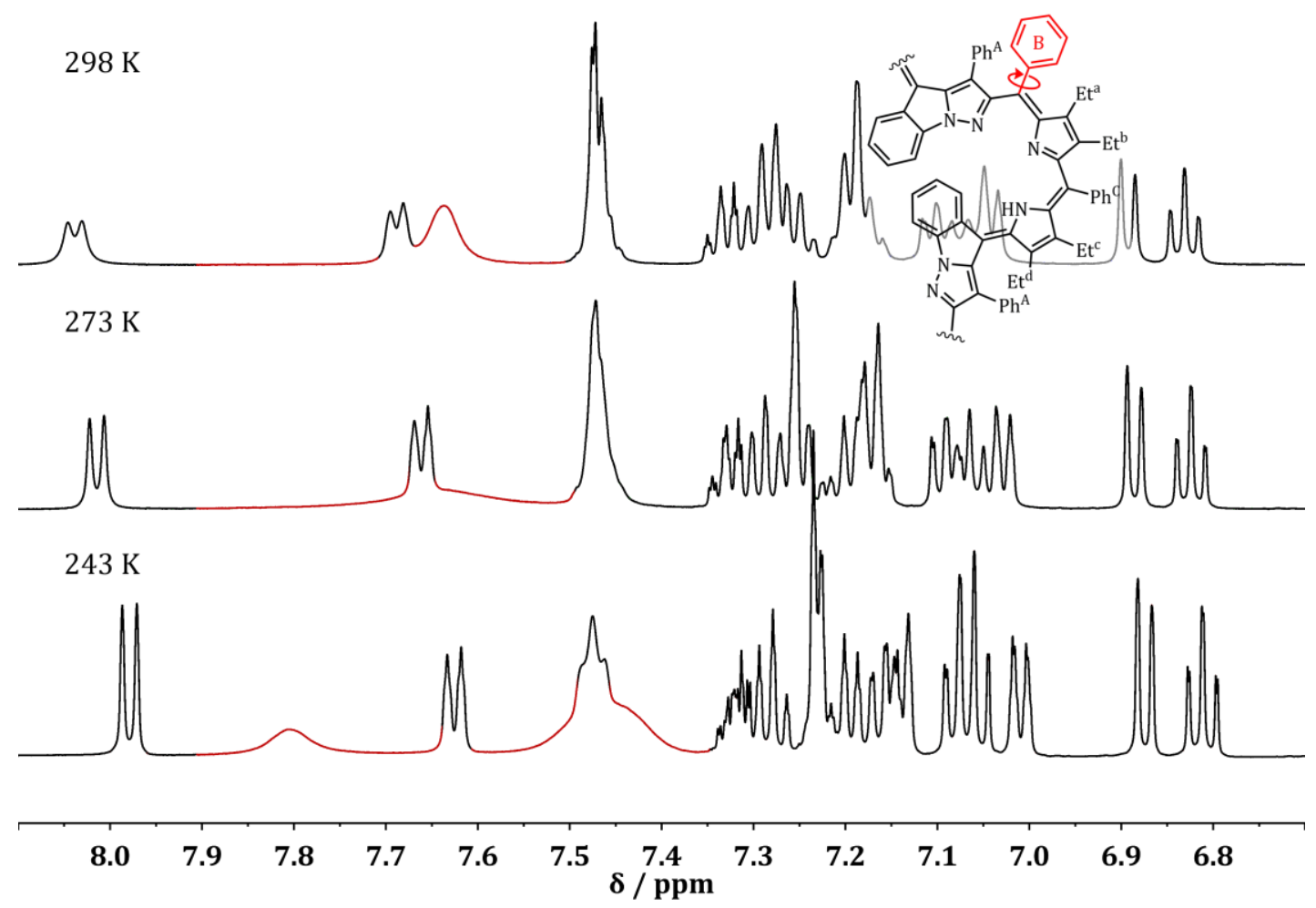

Figure S5: Section ( $\delta=8.1-6.7 \mathrm{ppm})$ of variable temperature ${ }^{1} \mathrm{H}$ NMR spectrum $\left(500 \mathrm{MHz}, \mathrm{CD}_{2} \mathrm{Cl}_{2}, 243 \mathrm{~K}\right)$ of $33 \mathrm{H}_{2}$. Temperatures are indicated in the spectrum. Broad signals highlighted in red show high rotational freedom of the meso-phenyl group $\mathrm{Ph}^{\mathrm{B}}$.

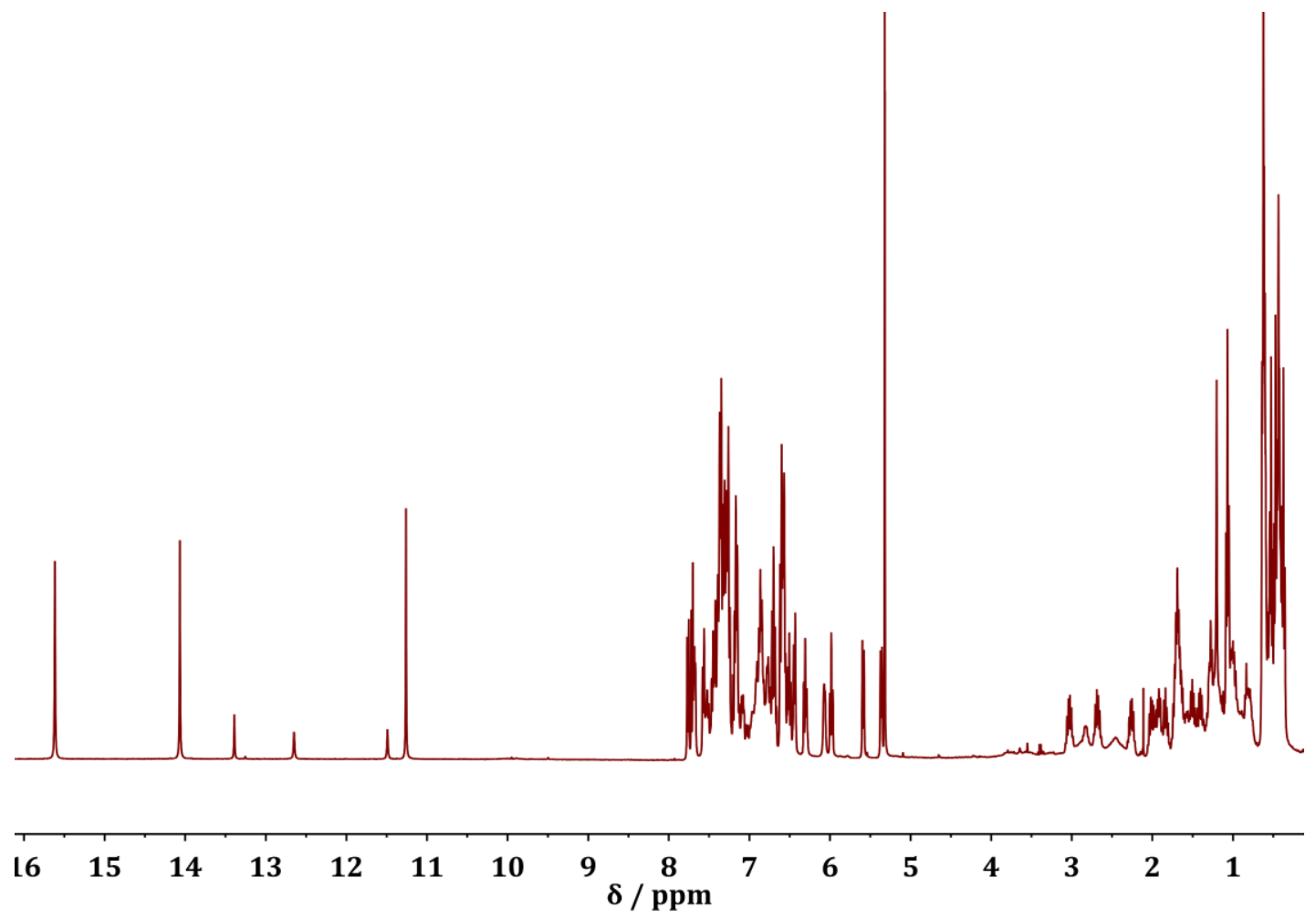

Figure S6: ${ }^{1} \mathrm{H}$ NMR spectrum $\left(400 \mathrm{MHz}, \mathrm{CD}_{2} \mathrm{Cl}_{2}, 243 \mathrm{~K}\right)$ of $\mathbf{3 2 H}_{3}$. 


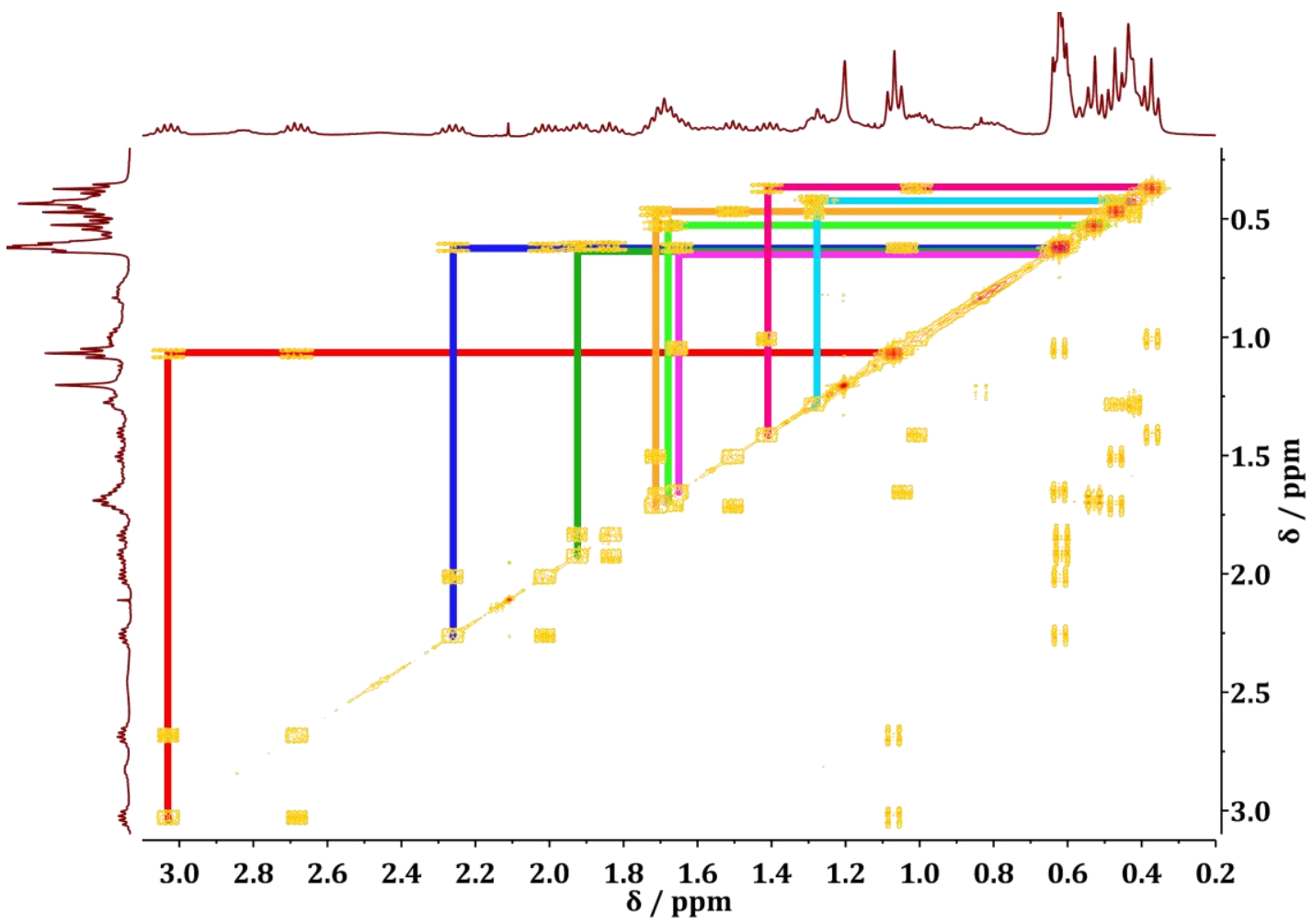

Figure S7: Section $(\delta=3.1-0.2 \mathrm{ppm})$ of the ${ }^{1} \mathrm{H}^{1} \mathrm{H}-\mathrm{COSY}$ spectrum $\left(400 \mathrm{MHz}, \mathrm{CD}_{2} \mathrm{Cl}_{2}, 243 \mathrm{~K}\right)$ of $\mathbf{3 2} \mathbf{H}_{3}$; correlated diastereotopic $\mathrm{CH}_{2}$-protons and associated $\mathrm{CH}_{3}$-signals are connected by colored lines.

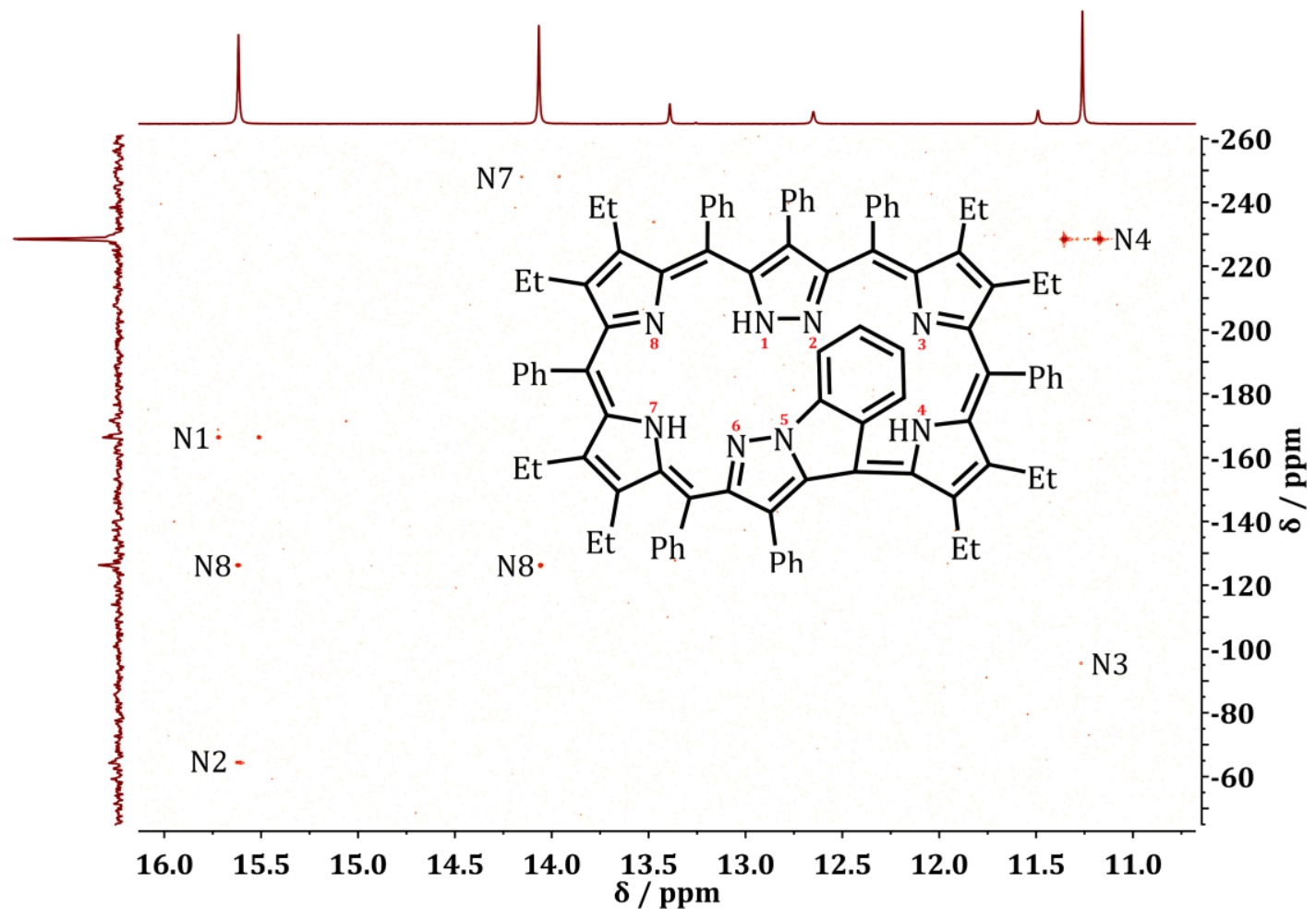

Figure S8: ${ }^{1} \mathrm{H}^{15} \mathrm{~N}$ HMBC spectrum $\left(400 \mathrm{MHz}, \mathrm{CD}_{2} \mathrm{Cl}_{2}, 243 \mathrm{~K}\right)$ of $\mathbf{3 2} \mathbf{H}_{3}$. Nitrogen atoms $\mathrm{N} 5$ and $\mathrm{N} 6$ of the spatially separated pyrazoloindole do not show any cross peaks. 

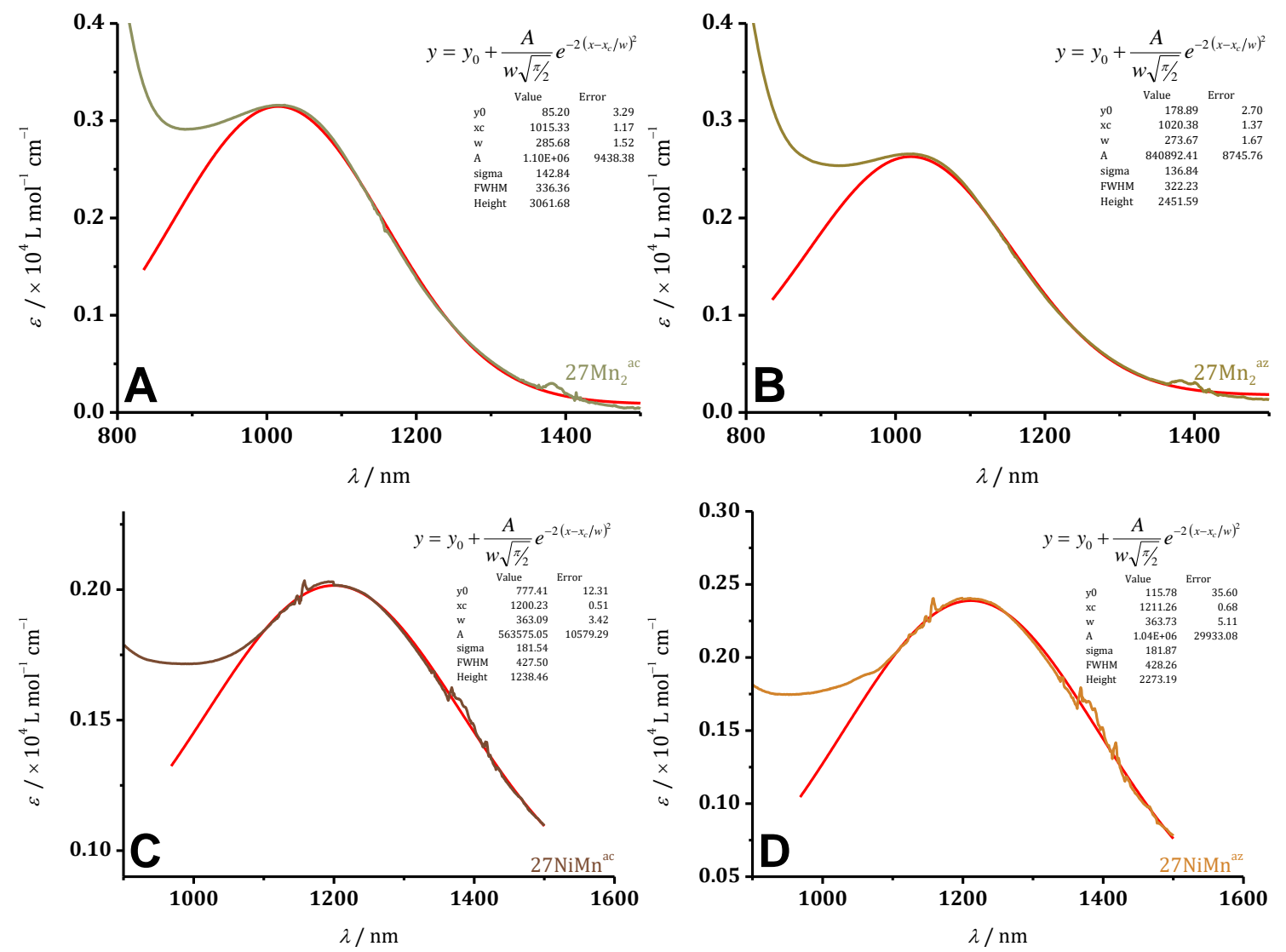

Figure S9: NIR section of the UV/vis spectra for complexes $\mathbf{2 7} \mathbf{M n}_{2^{\mathbf{a c}}}(\mathrm{A}), \mathbf{2 7} \mathbf{M n}_{\mathbf{2}^{\mathrm{az}}}(\mathrm{B}), \mathbf{2 7} \mathbf{N i M n} \mathbf{a c}^{\mathrm{ac}}(\mathrm{C})$ and $\mathbf{2 7} \mathbf{N i M n}{ }^{\mathrm{az}}$ (D). Curve fits (red trace) and parameters were calculated using the indicated Gaussian fitting function.

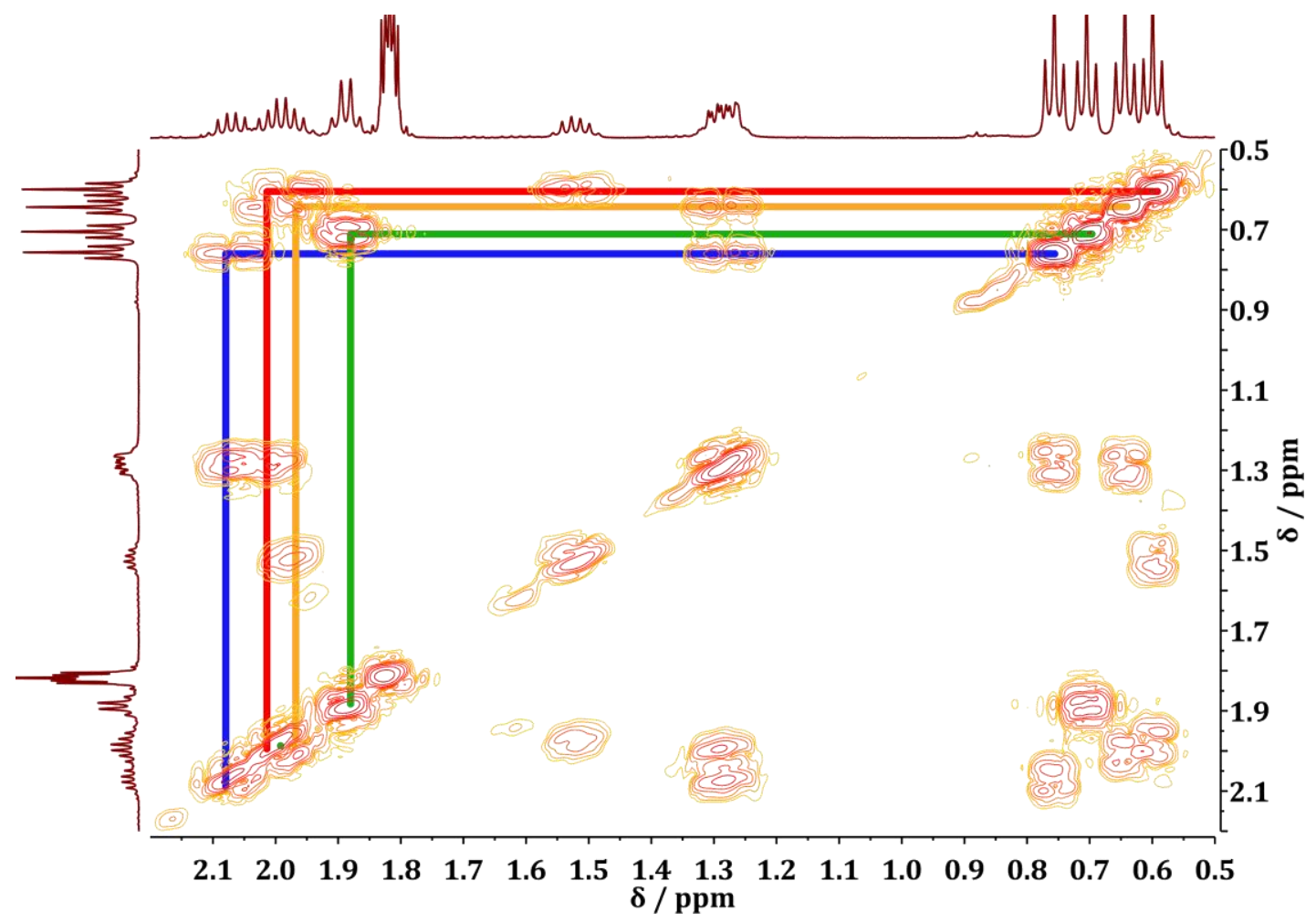

Figure S10: Section $(\delta=2.2-0.5 \mathrm{ppm})$ of ${ }^{1} \mathrm{H}^{1} \mathrm{H}-\mathrm{COSY}$ spectrum $\left(500 \mathrm{MHz}, \mathrm{CD}_{2} \mathrm{Cl}_{2}, 298 \mathrm{~K}\right)$ of palladium complex $\mathbf{2 7} \mathbf{H}_{2} \mathbf{P d}$ in its neutral form. Correlated spin systems are connected by colored lines. 


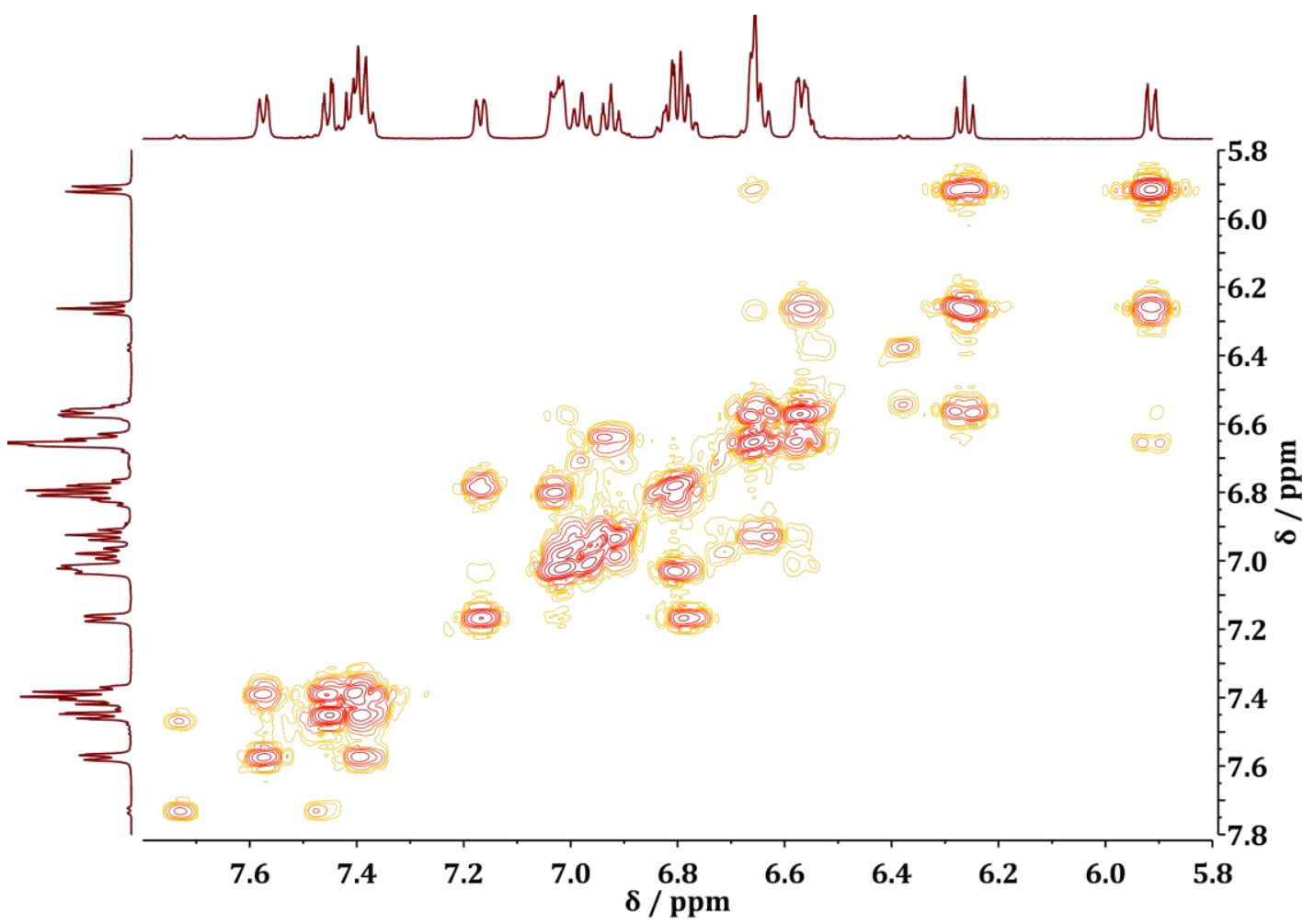

Figure S11: Section $(\delta=8.8-5.8 \mathrm{ppm})$ of ${ }^{1} \mathrm{H}^{1} \mathrm{H}-\mathrm{COSY}$ spectrum $\left(500 \mathrm{MHz}, \mathrm{CD}_{2} \mathrm{Cl}_{2}, 298 \mathrm{~K}\right)$ of palladium complex $\mathbf{2 7} \mathbf{H}_{2} \mathbf{P d}$ in its neutral form.

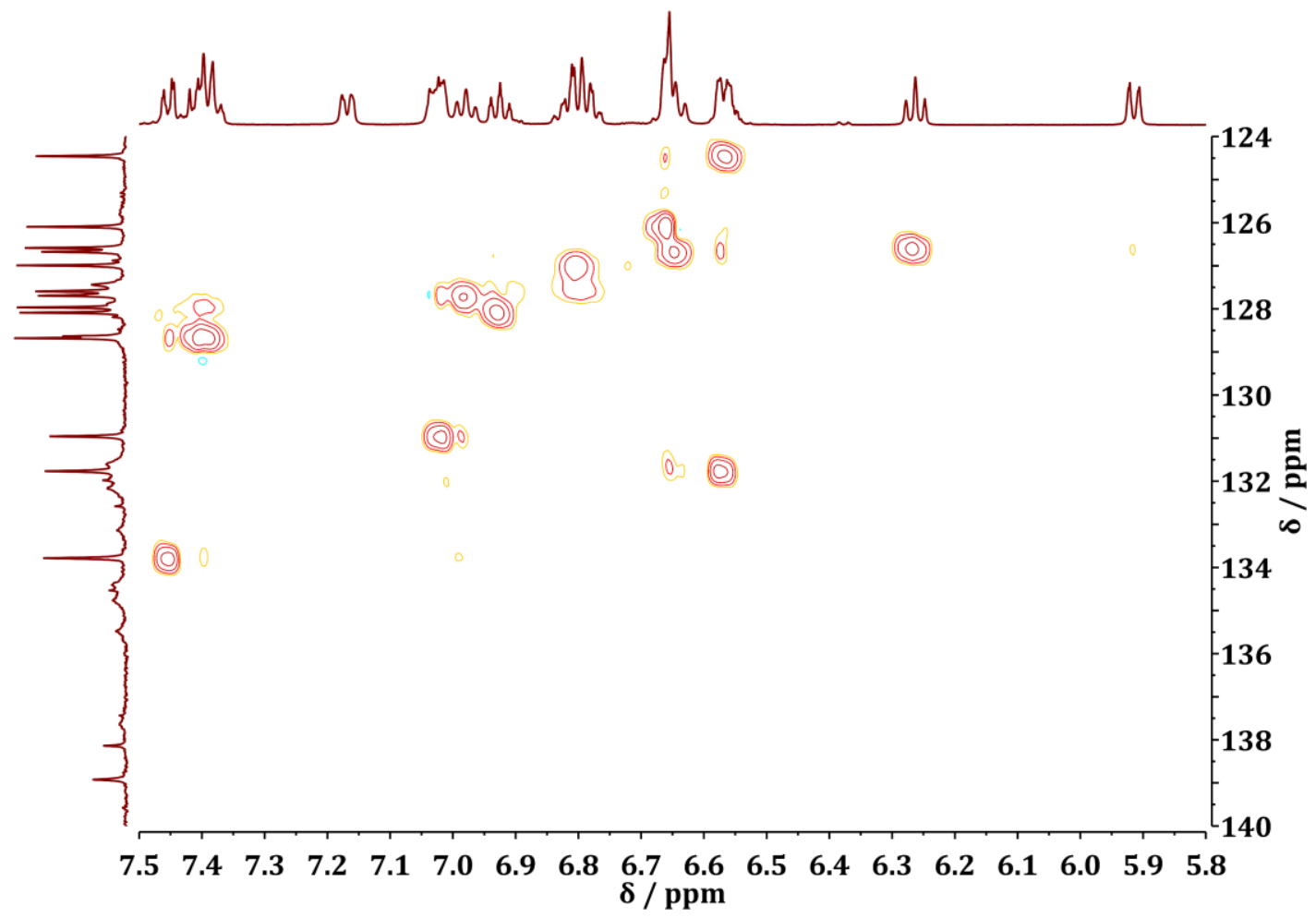

Figure S12: Section $(\delta=7.5-5.8$ and $140.0-124.0 \mathrm{ppm})$ of ${ }^{1} \mathrm{H}^{13} \mathrm{C}-\mathrm{HSQC}$ spectrum $\left(500 \mathrm{MHz}, \mathrm{CD}_{2} \mathrm{Cl}_{2}, 298 \mathrm{~K}\right)$ of palladium complex $\mathbf{2 7} \mathbf{H}_{2} \mathbf{P d}$ in its neutral form. 


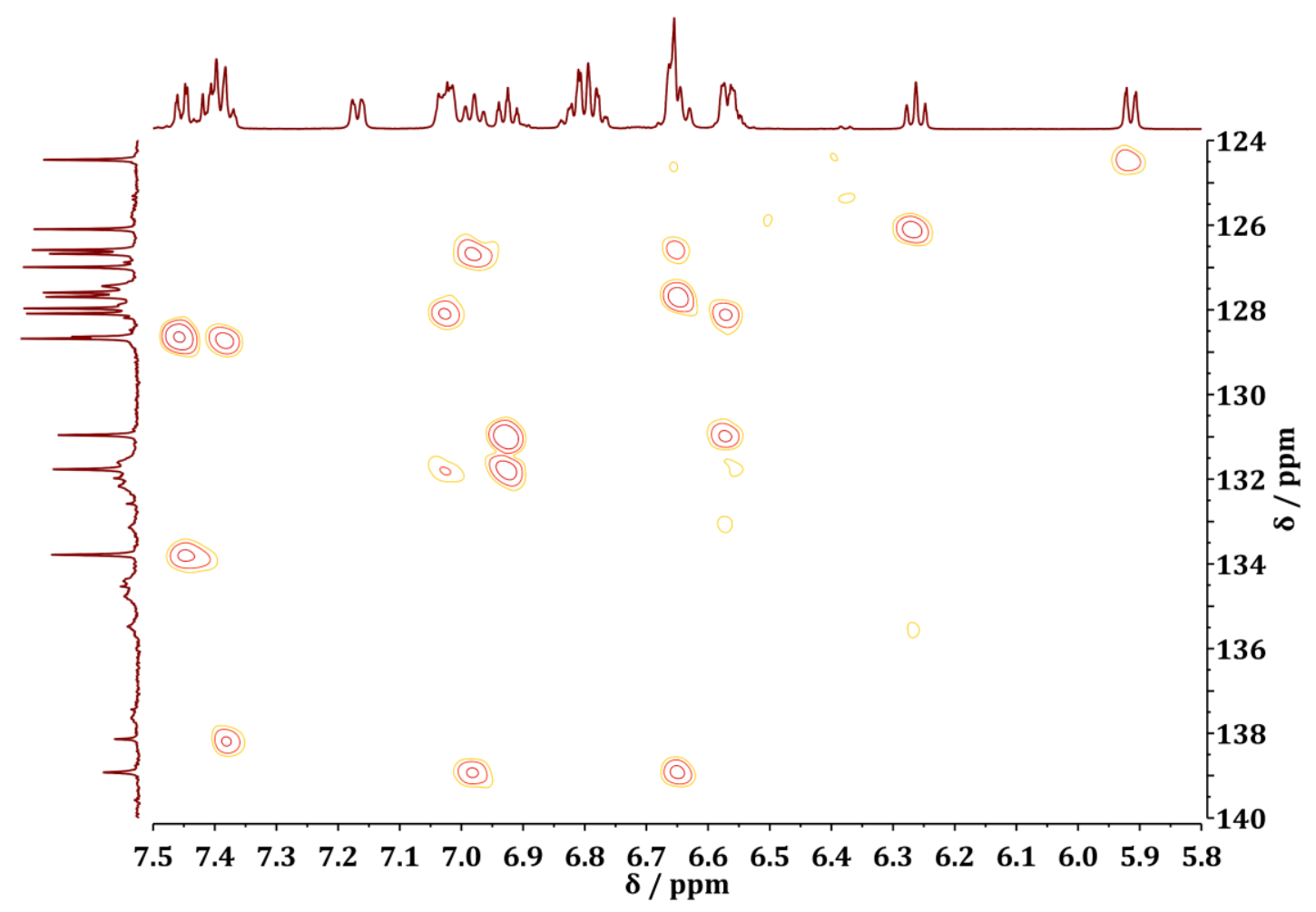

Figure S13: Section $(\delta=7.5-5.8$ and $140.0-124.0 \mathrm{ppm})$ of ${ }^{1} \mathrm{H}^{13} \mathrm{C}-\mathrm{HMBC}$ spectrum $\left(500 \mathrm{MHz}, \mathrm{CD}_{2} \mathrm{Cl}_{2}, 298 \mathrm{~K}\right)$ of palladium complex $\mathbf{2 7} \mathbf{H}_{2} \mathbf{P d}$ in its neutral form.

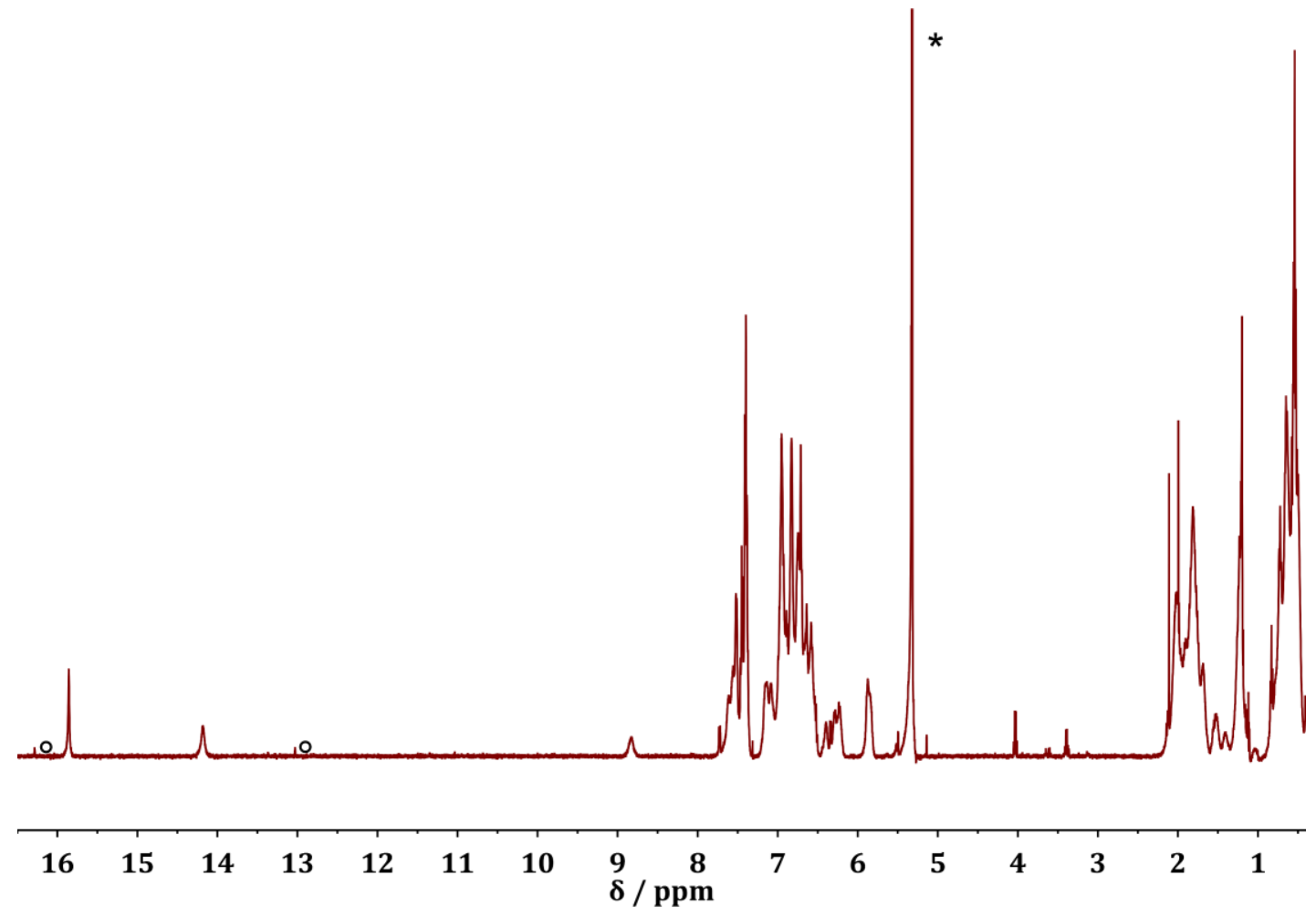

Figure S14: ${ }^{1} \mathrm{H}$ NMR spectrum ( $500 \mathrm{MHz}, \mathrm{CH}_{2} \mathrm{Cl}_{2}, 238 \mathrm{~K}$ ) of the monoprotonated monopalladium complex $\mathbf{2 7} \mathbf{H}_{3} \mathbf{P d}^{+}$. Residual proton signals of $\mathrm{CD}_{2} \mathrm{Cl}_{2}$ are marked with an asterisk (*). Signals marked with a circle (०) show the presence of the diprotonated species due to limited accuracy during the titration. 


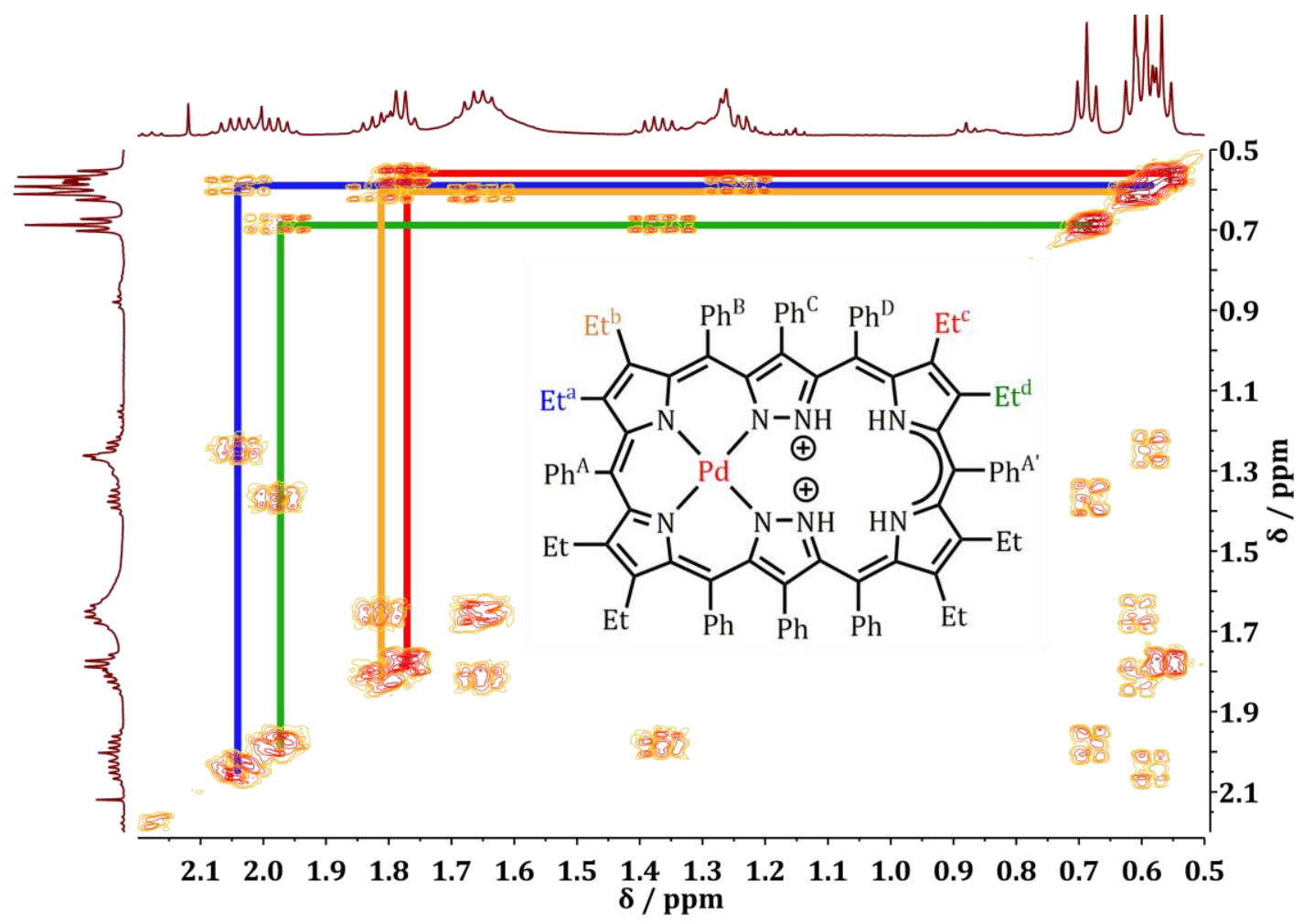

Figure S15: Section ( $\delta=2.2-0.5 \mathrm{ppm})$ of the ${ }^{1} \mathrm{H}^{1} \mathrm{H}-\mathrm{COSY}$ spectrum $\left(500 \mathrm{MHz}, \mathrm{CH}_{2} \mathrm{Cl}_{2}, 298 \mathrm{~K}\right)$ of the diprotonated palladium complex $\mathbf{2 7} \mathbf{H}_{4} \mathbf{P d}^{2+}$. Correlating spin systems are indicated in color according to inset structure.

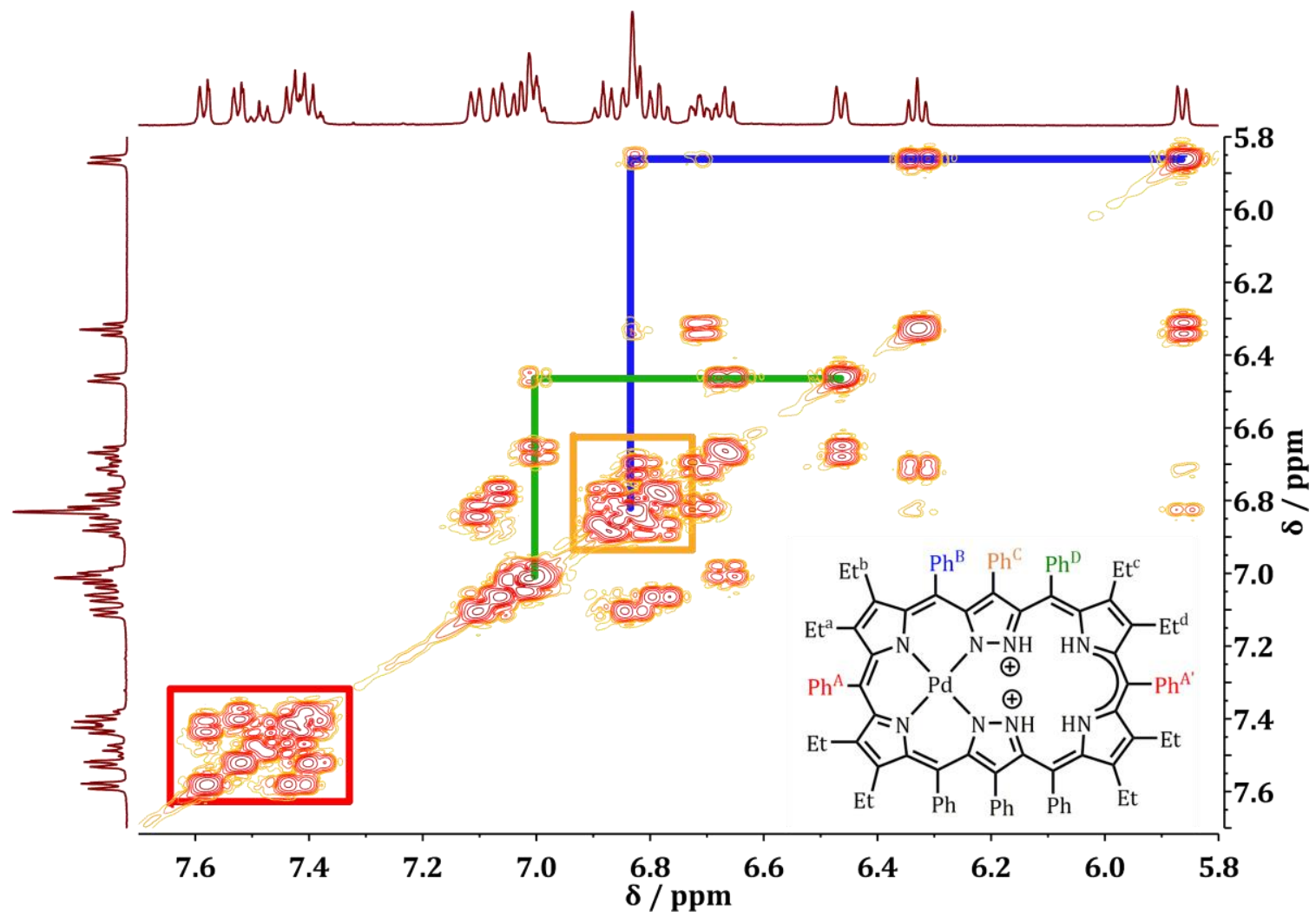

Figure S16: Section $(\delta=7.7-5.8 \mathrm{ppm})$ of the ${ }^{1} \mathrm{H}^{1} \mathrm{H}-\mathrm{COSY}$ spectrum $\left(500 \mathrm{MHz}, \mathrm{CH}_{2} \mathrm{Cl}_{2}, 298 \mathrm{~K}\right)$ of the diprotonated monopalladium complex $\mathbf{2 7} \mathbf{H}_{4} \mathbf{P d}^{2+}$. Correlating spin systems are indicated in color according to inset structure. 


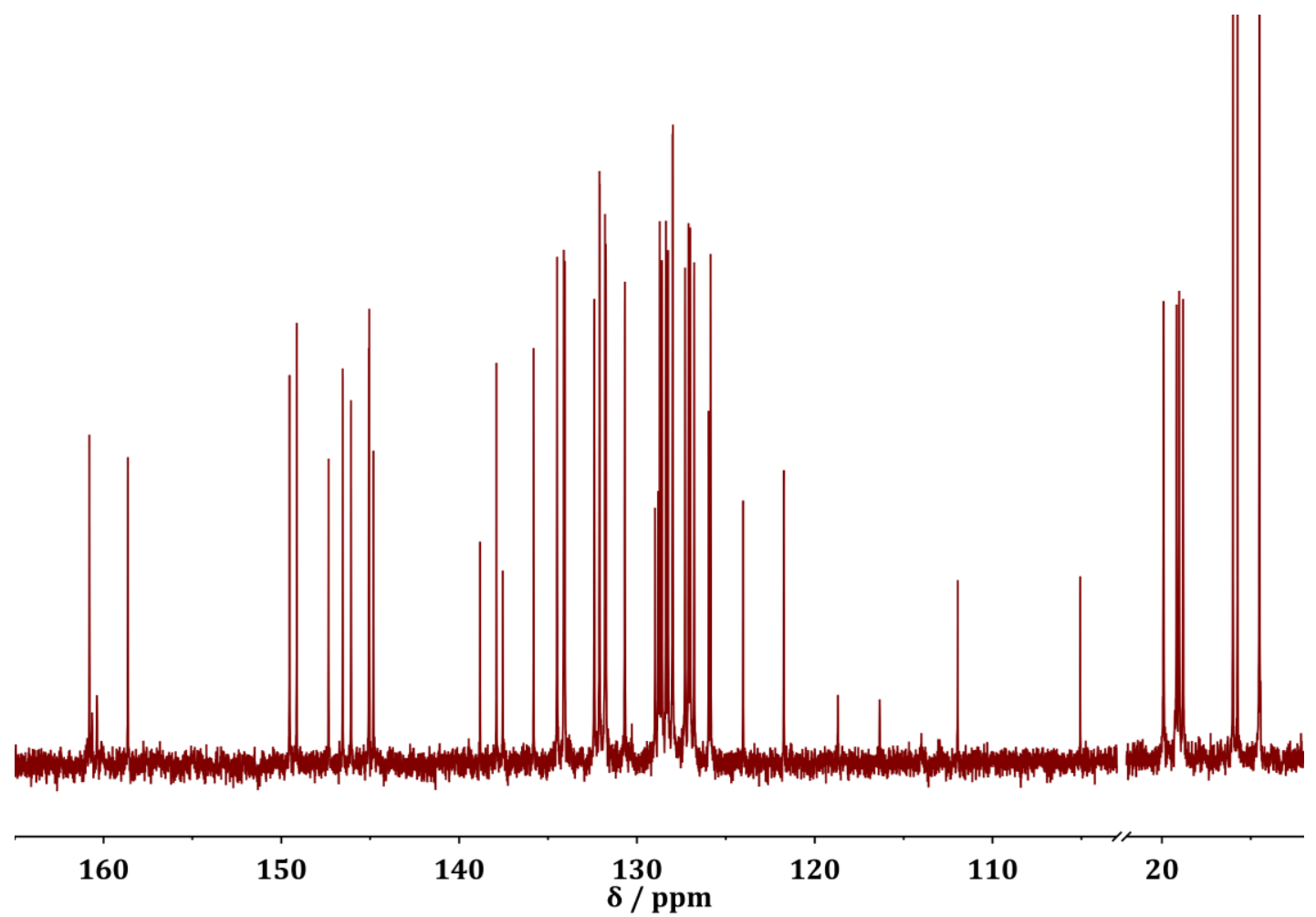

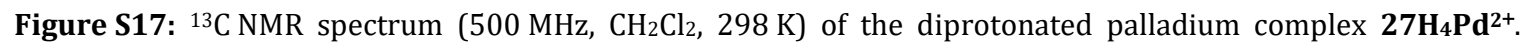
Section $(\delta=103-23 \mathrm{ppm})$ not shown.

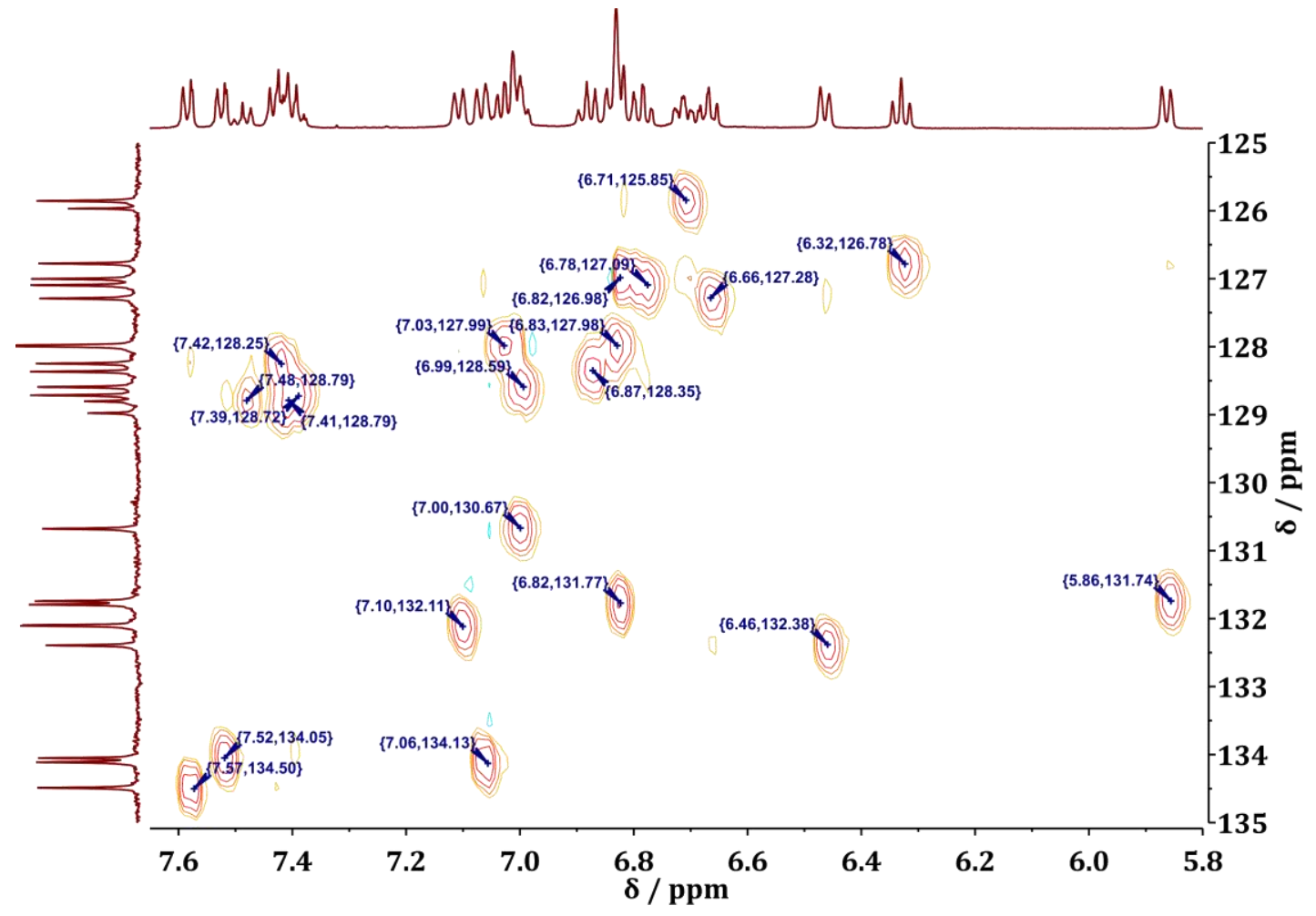

Figure S18: Section ( $\delta=7.65-5.8$ and $135-125 \mathrm{ppm}$ ) of the ${ }^{1} \mathrm{H}^{13} \mathrm{C}-\mathrm{HSQC}$ spectrum $\left(500 \mathrm{MHz}, \mathrm{CH}_{2} \mathrm{Cl}_{2}, 293 \mathrm{~K}\right.$ ) of the diprotonated palladium complex $\mathbf{2 7} \mathbf{H}_{4} \mathbf{P d}^{2+}$. 


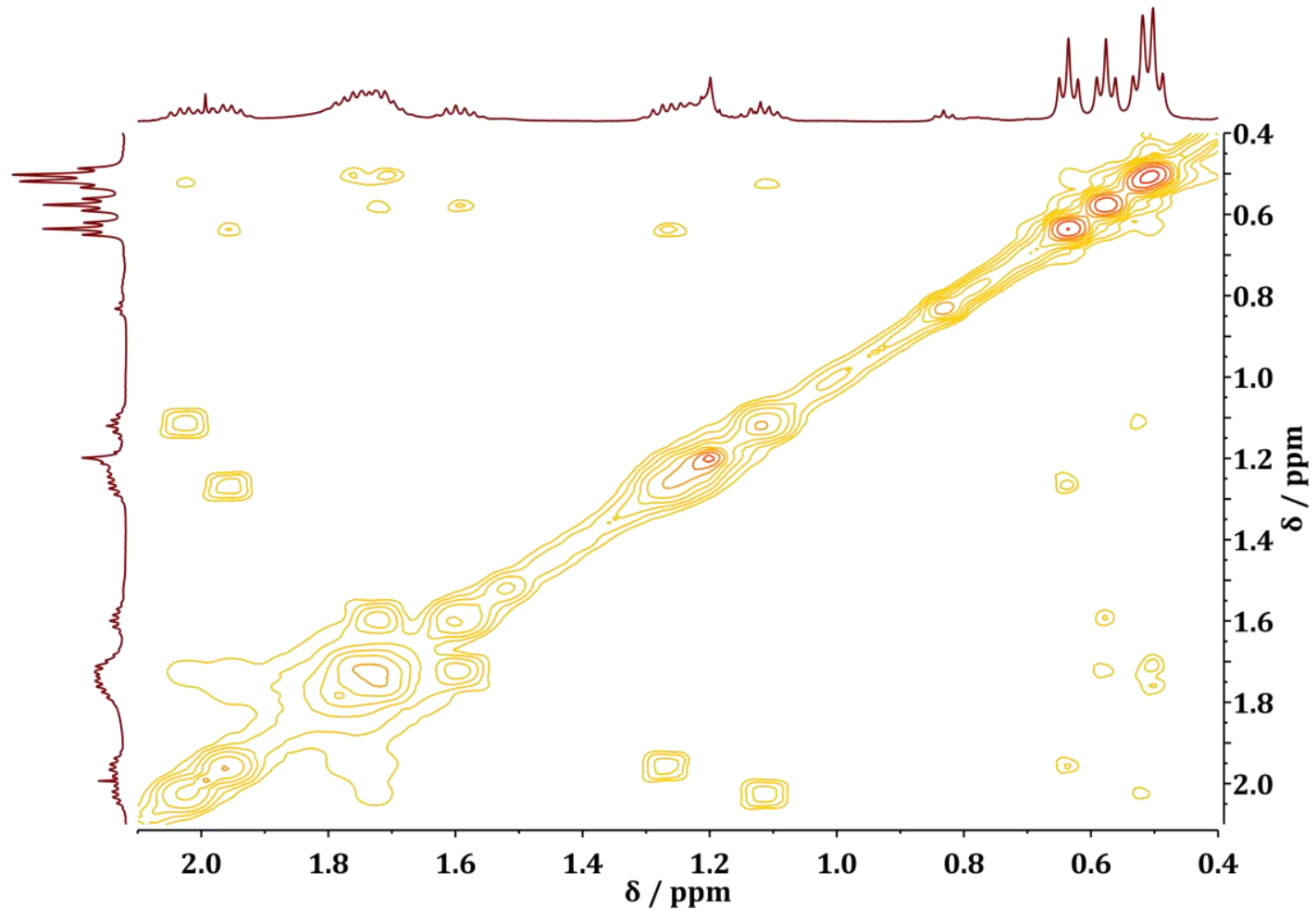

Figure S19: Section $(\delta=2.1-0.4 \mathrm{ppm})$ of the NOESY spectrum $\left(500 \mathrm{MHz}, \mathrm{CH}_{2} \mathrm{Cl}_{2}, 238 \mathrm{~K}\right)$ of the diprotonated monopalladium complex $\mathbf{2 7} \mathbf{H}_{4} \mathbf{P d}^{2+}$.

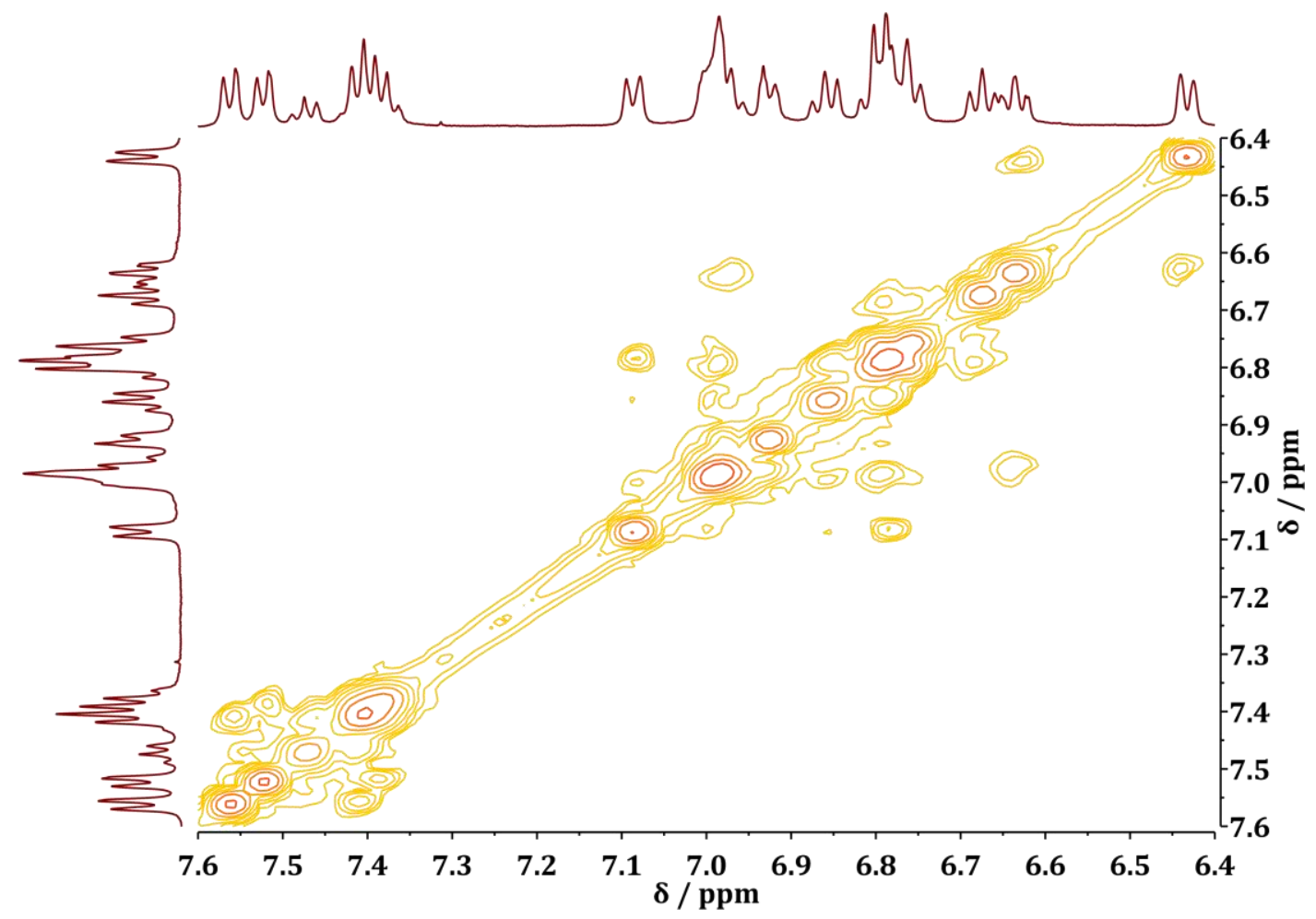

Figure S20: Section $(\delta=7.6-6.4 \mathrm{ppm})$ of the NOESY spectrum $\left(500 \mathrm{MHz}, \mathrm{CH}_{2} \mathrm{Cl}_{2}, 238 \mathrm{~K}\right)$ of the diprotonated monopalladium complex $\mathbf{2 7} \mathbf{H}_{4} \mathbf{P d}^{2+}$. 


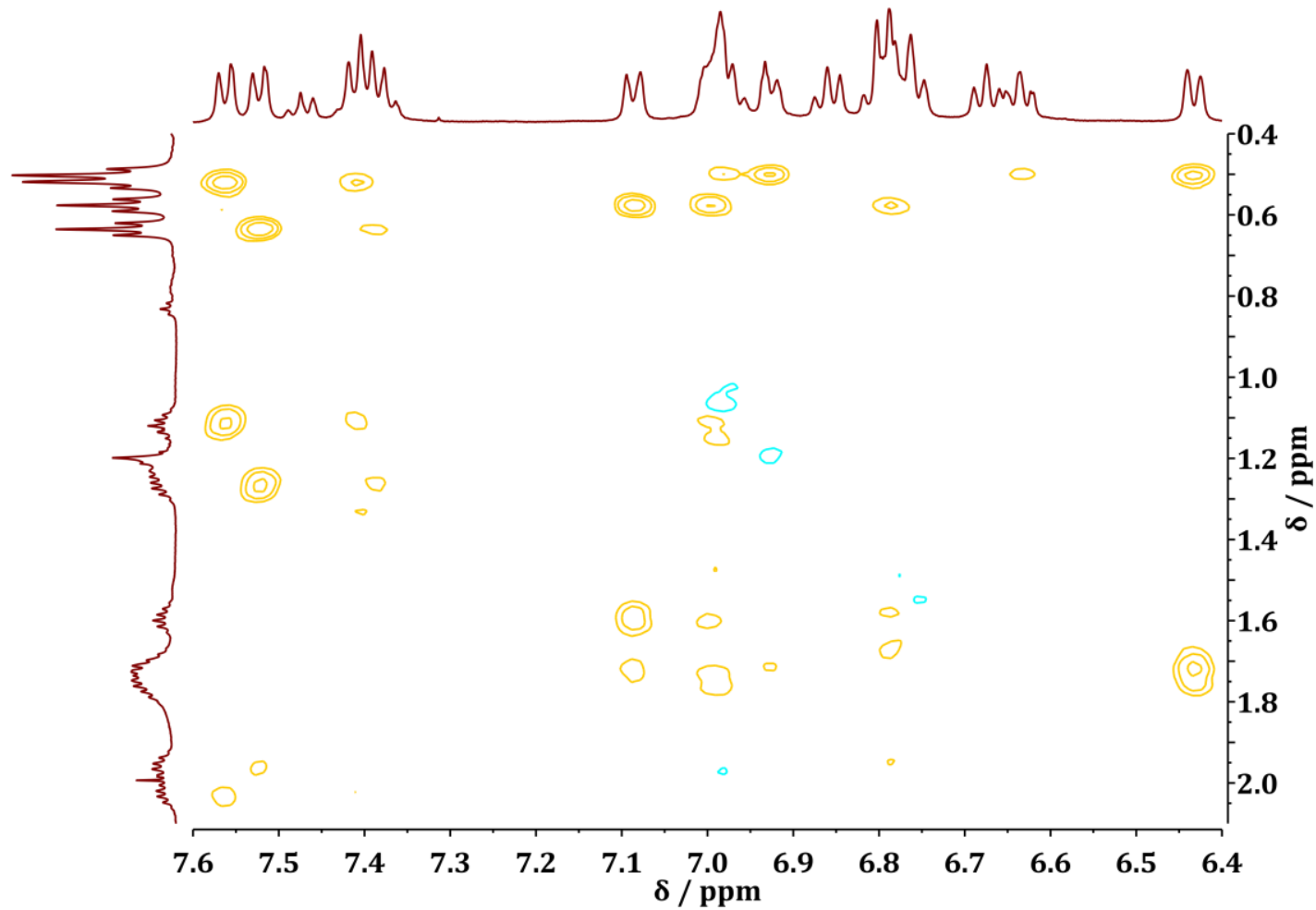

Figure S21: Section ( $\delta=7.6-6.4$ and $2.1-0.4 \mathrm{ppm})$ of the NOESY spectrum $\left(500 \mathrm{MHz}, \mathrm{CH}_{2} \mathrm{Cl}_{2}, 238 \mathrm{~K}\right)$ of the diprotonated monopalladium complex $2 \mathbf{2 7} \mathbf{H}_{4} \mathbf{P d}^{2+}$. 



\subsection{Computational Details}

Computations were performed using the ORCA software package 3.0.3.[146] Geometry optimizations were performed using the BP86 functional[147] with def2-TZVP basis set and def2-TZVP/J auxiliary set ${ }^{[148,149]}$ for all atoms. Subsequent single point calculations were run with the B3LYP functional.[150] The def2-TZVPP basis set and def2-TZVPP/J auxiliary set $[148,149,151]$ were employed for all atoms. An effective core potential approach ECP was applied to Pd and the RIJCOSX approximation [152] was used. Initial coordinates were taken from the experimental crystal structure, and protonation patterns and charges were modified for the respective calculations.

\subsubsection{Optimized Molecular Structures}

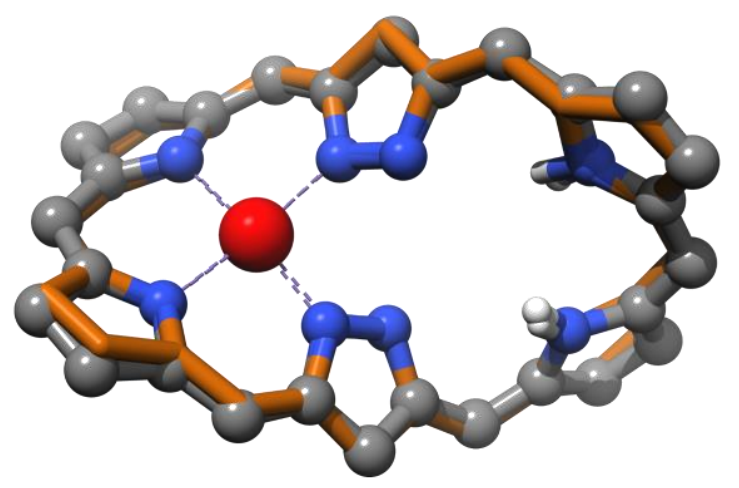

Final Single Point Energy: -4119.987927520 478 Hartrees (no imaginary frequences)

Figure S22: Overlay of the cis-tautomers of $\mathbf{2 7} \mathbf{H}_{2} \mathbf{P d}$ before (ball-and-stick model; palladium in red, carbon in grey, nitrogen in blue, hydrogen in white) and after geometry optimization (wire model; palladium in red, carbon in orange, nitrogen in blue, and hydrogen in white).[93] Peripheral substituents were omitted for clarity.

Table 12: Optimized CARTESIAN coordinates for the cis-tautomer of $\mathbf{2 7} \mathbf{H}_{2} \mathbf{P d}$.

\begin{tabular}{rrrrrrrr}
\hline & \multicolumn{1}{c}{$X$} & \multicolumn{1}{c}{$Z$} & & \multicolumn{1}{c}{$X$} & \multicolumn{1}{l}{$y$} & \multicolumn{1}{c}{$Z$} \\
\hline $\mathrm{C}$ & -0.201696 & 2.00183 & -15.168113 & $\mathrm{C}$ & 2.740198 & -4.5062 & -8.22407 \\
$\mathrm{C}$ & -1.47689 & 1.930534 & -15.801916 & $\mathrm{H}$ & 2.712936 & -5.404156 & -7.60409 \\
$\mathrm{C}$ & -1.914628 & 0.60709 & -15.542663 & $\mathrm{C}$ & 3.75083 & -3.55958 & -8.041636 \\
$\mathrm{C}$ & -3.096738 & -0.110844 & -15.964185 & $\mathrm{H}$ & 4.510564 & -3.708352 & -7.272268 \\
$\mathrm{C}$ & -3.612159 & -1.166036 & -15.21203 & $\mathrm{C}$ & 3.789024 & -2.425519 & -8.859936 \\
$\mathrm{C}$ & -4.695406 & -2.105339 & -15.52733 & $\mathrm{H}$ & 4.576572 & -1.681125 & -8.726523 \\
$\mathrm{C}$ & -4.986222 & -2.789453 & -14.365079 & $\mathrm{C}$ & 2.823774 & -2.242506 & -9.85016 \\
$\mathrm{C}$ & -4.050719 & -2.318521 & -13.338193 & $\mathrm{H}$ & 2.853998 & -1.356883 & -10.487653 \\
$\mathrm{C}$ & -3.987335 & -2.6781 & -11.966448 & $\mathrm{C}$ & 3.251665 & -4.588994 & -12.483815 \\
$\mathrm{C}$ & -2.778865 & -2.593535 & -11.227965 & $\mathrm{C}$ & 2.718694 & -5.800283 & -12.005565 \\
$\mathrm{C}$ & -2.609206 & -2.502131 & -9.775888 & $\mathrm{H}$ & 1.638355 & -5.952941 & -12.042062 \\
$\mathrm{C}$ & -1.25892 & -2.575895 & -9.514128 & $\mathrm{C}$ & 3.549742 & -6.805825 & -11.511107 \\
$\mathrm{C}$ & -0.576926 & -2.712622 & -10.805965 & $\mathrm{H}$ & 3.11316 & -7.739566 & -11.151609 \\
$\mathrm{C}$ & 0.767274 & -2.937976 & -11.090045 & $\mathrm{C}$ & 4.935016 & -6.62322 & -11.479228 \\
$\mathrm{C}$ & 1.269199 & -2.893678 & -12.445695 & $\mathrm{H}$ & 5.58533 & -7.409577 & -11.092505 \\
$\mathrm{C}$ & 2.373611 & -3.54594 & -13.044414 & $\mathrm{C}$ & 5.479939 & -5.425263 & -11.949062
\end{tabular}




\begin{tabular}{|c|c|c|c|c|c|c|c|}
\hline $\mathrm{C}$ & 2.425983 & -2.995905 & -14.358379 & $\mathrm{H}$ & 6.560274 & -5.268767 & -11.923953 \\
\hline $\mathrm{C}$ & 3.287462 & -3.321599 & -15.481477 & $\mathrm{C}$ & 4.647711 & -4.421198 & -12.446926 \\
\hline $\mathrm{C}$ & 3.775766 & -2.306961 & -16.308587 & $\mathrm{H}$ & 5.079518 & -3.48412 & -12.803036 \\
\hline $\mathrm{C}$ & 4.365942 & -2.311376 & -17.632197 & $\mathrm{C}$ & 3.622609 & -4.737554 & -15.728381 \\
\hline $\mathrm{C}$ & 4.858809 & -1.035572 & -17.887714 & $\mathrm{C}$ & 2.613073 & -5.719229 & -15.678996 \\
\hline $\mathrm{C}$ & 4.542584 & -0.195413 & -16.750404 & $\mathrm{H}$ & 1.588966 & -5.413499 & -15.459485 \\
\hline $\mathrm{C}$ & 4.867455 & 1.166364 & -16.485175 & $\mathrm{C}$ & 2.907106 & -7.058381 & -15.931851 \\
\hline $\mathrm{C}$ & 4.021055 & 2.019205 & -15.717918 & $\mathrm{H}$ & 2.107981 & -7.801032 & -15.905216 \\
\hline $\mathrm{C}$ & 4.35222 & 3.175628 & -14.911097 & $\mathrm{C}$ & 4.219264 & -7.449988 & -16.215104 \\
\hline $\mathrm{C}$ & 3.165712 & 3.777367 & -14.504857 & $\mathrm{H}$ & 4.450591 & -8.499614 & -16.403439 \\
\hline $\mathrm{C}$ & 2.065891 & 2.98874 & -15.023703 & $\mathrm{C}$ & 5.236815 & -6.490956 & -16.240257 \\
\hline $\mathrm{C}$ & 0.677421 & 3.139485 & -14.96437 & $\mathrm{H}$ & 6.267045 & -6.792064 & -16.437643 \\
\hline $\mathrm{C}$ & -2.137728 & 3.016986 & -16.547349 & $\mathrm{C}$ & 4.941932 & -5.149843 & -16.001317 \\
\hline $\mathrm{C}$ & -1.471536 & 3.664991 & -17.605075 & $\mathrm{H}$ & 5.739113 & -4.404695 & -16.00254 \\
\hline $\mathrm{H}$ & -0.474567 & 3.325181 & -17.890349 & $\mathrm{C}$ & 4.221088 & -3.394884 & -18.667058 \\
\hline $\mathrm{C}$ & -2.0724 & 4.716147 & -18.29882 & $\mathrm{H}$ & 4.893105 & -3.176981 & -19.50893 \\
\hline $\mathrm{H}$ & -1.538059 & 5.19792 & -19.119777 & $\mathrm{H}$ & 4.531356 & -4.367918 & -18.267255 \\
\hline $\mathrm{C}$ & -3.354858 & 5.146817 & -17.949638 & $\mathrm{C}$ & 2.776066 & -3.508065 & -19.192578 \\
\hline $\mathrm{H}$ & -3.826395 & 5.968319 & -18.491345 & $\mathrm{H}$ & 2.077974 & -3.761109 & -18.383329 \\
\hline $\mathrm{C}$ & -4.02878 & 4.515326 & -16.90039 & $\mathrm{H}$ & 2.44013 & -2.561483 & -19.640292 \\
\hline $\mathrm{H}$ & -5.028686 & 4.846464 & -16.615486 & $\mathrm{H}$ & 2.708362 & -4.292895 & -19.960071 \\
\hline $\mathrm{C}$ & -3.427829 & 3.46352 & -16.208354 & $\mathrm{C}$ & 5.437742 & -0.572025 & -19.200252 \\
\hline $\mathrm{H}$ & -3.953258 & 2.985639 & -15.380528 & $\mathrm{H}$ & 4.737914 & -0.854799 & -20.004967 \\
\hline $\mathrm{C}$ & -3.763475 & 0.346337 & -17.210813 & $\mathrm{H}$ & 5.483253 & 0.525156 & -19.211332 \\
\hline $\mathrm{C}$ & -3.04749 & 0.427788 & -18.418394 & $\mathrm{C}$ & 6.828869 & -1.138387 & -19.537817 \\
\hline $\mathrm{H}$ & -1.995799 & 0.137525 & -18.4307 & $\mathrm{H}$ & 6.826083 & -2.237586 & -19.555559 \\
\hline $\mathrm{C}$ & -3.668162 & 0.859676 & -19.591202 & $\mathrm{H}$ & 7.156267 & -0.785511 & -20.526605 \\
\hline $\mathrm{H}$ & -3.097513 & 0.904033 & -20.520624 & $\mathrm{H}$ & 7.575693 & -0.814835 & -18.801268 \\
\hline $\mathrm{C}$ & -5.014113 & 1.237901 & -19.577944 & $\mathrm{C}$ & 6.127729 & 1.726106 & -17.025861 \\
\hline $\mathrm{H}$ & -5.495575 & 1.584889 & -20.493506 & $\mathrm{C}$ & 7.352216 & 1.04466 & -16.872842 \\
\hline $\mathrm{C}$ & -5.734553 & 1.179581 & -18.381612 & $\mathrm{H}$ & 7.362263 & 0.088125 & -16.347579 \\
\hline $\mathrm{H}$ & -6.781712 & 1.48594 & -18.357513 & $\mathrm{C}$ & 8.544933 & 1.588875 & -17.352545 \\
\hline $\mathrm{C}$ & -5.115088 & 0.738117 & -17.211802 & $\mathrm{H}$ & 9.482395 & 1.048858 & -17.207669 \\
\hline $\mathrm{H}$ & -5.674227 & 0.697047 & -16.275001 & $\mathrm{C}$ & 8.544628 & 2.825612 & -18.004054 \\
\hline C & -5.244503 & -2.452457 & -16.885106 & $\mathrm{H}$ & 9.477154 & 3.250635 & -18.377553 \\
\hline $\mathrm{H}$ & -6.123388 & -3.100036 & -16.751534 & $\mathrm{C}$ & 7.337463 & 3.514202 & -18.171159 \\
\hline $\mathrm{H}$ & -5.603065 & -1.562227 & -17.415199 & $\mathrm{H}$ & 7.324108 & 4.476588 & -18.685594 \\
\hline $\mathrm{C}$ & -4.220517 & -3.182389 & -17.776753 & $\mathrm{C}$ & 6.147117 & 2.972854 & -17.68746 \\
\hline $\mathrm{H}$ & -3.859147 & -4.101606 & -17.294218 & $\mathrm{H}$ & 5.207754 & 3.511088 & -17.826591 \\
\hline $\mathrm{H}$ & -4.673729 & -3.456763 & -18.740409 & $\mathrm{C}$ & 5.724452 & 3.513932 & -14.391739 \\
\hline $\mathrm{H}$ & -3.349204 & -2.545955 & -17.98096 & $\mathrm{H}$ & 6.434058 & 3.654133 & -15.219634 \\
\hline $\mathrm{C}$ & -5.919853 & -3.96493 & -14.250834 & $\mathrm{H}$ & 5.676882 & 4.476986 & -13.865401 \\
\hline $\mathrm{H}$ & -6.511583 & -3.90626 & -13.329563 & $\mathrm{C}$ & 6.273626 & 2.445545 & -13.427299 \\
\hline $\mathrm{H}$ & -6.644625 & -3.9298 & -15.078602 & $\mathrm{H}$ & 5.610409 & 2.320531 & -12.559448 \\
\hline $\mathrm{C}$ & -5.185388 & -5.31886 & -14.28742 & $\mathrm{H}$ & 6.364339 & 1.470751 & -13.925511 \\
\hline
\end{tabular}




\begin{tabular}{|c|c|c|c|c|c|c|c|}
\hline $\mathrm{H}$ & -5.901744 & -6.150966 & -14.219114 & $\mathrm{H}$ & 7.2689 & 2.731971 & -13.058841 \\
\hline $\mathrm{H}$ & -4.613285 & -5.433243 & -15.219136 & $\mathrm{C}$ & 3.079901 & 4.925615 & -13.532511 \\
\hline $\mathrm{H}$ & -4.478838 & -5.409733 & -13.45024 & $\mathrm{H}$ & 2.051767 & 5.02224 & -13.163163 \\
\hline $\mathrm{C}$ & -5.219937 & -3.159537 & -11.292234 & $\mathrm{H}$ & 3.696857 & 4.673589 & -12.652996 \\
\hline $\mathrm{C}$ & -6.404261 & -2.397732 & -11.324161 & $\mathrm{C}$ & 3.550781 & 6.282645 & -14.086902 \\
\hline $\mathrm{H}$ & -6.400302 & -1.440631 & -11.848144 & $\mathrm{H}$ & 4.589496 & 6.239612 & -14.442185 \\
\hline $\mathrm{C}$ & -7.567661 & -2.848054 & -10.69802 & $\mathrm{H}$ & 2.926165 & 6.605758 & -14.929634 \\
\hline $\mathrm{H}$ & -8.471876 & -2.237178 & -10.730944 & $\mathrm{H}$ & 3.490969 & 7.056014 & -13.307146 \\
\hline $\mathrm{C}$ & -7.576107 & -4.074964 & -10.026674 & $\mathrm{C}$ & 0.057759 & 4.44185 & -14.645358 \\
\hline $\mathrm{H}$ & -8.485764 & -4.428387 & -9.538787 & $\mathrm{C}$ & 0.417124 & 5.620486 & -15.324943 \\
\hline $\mathrm{C}$ & -6.408825 & -4.845121 & -9.986008 & $\mathrm{H}$ & 1.149848 & 5.562018 & -16.131138 \\
\hline $\mathrm{H}$ & -6.406286 & -5.806964 & -9.470218 & $\mathrm{C}$ & -0.181063 & 6.839679 & -15.007439 \\
\hline $\mathrm{C}$ & -5.245127 & -4.391059 & -10.609463 & $\mathrm{H}$ & 0.098398 & 7.73974 & -15.557358 \\
\hline $\mathrm{H}$ & -4.33703 & -4.995858 & -10.578119 & $\mathrm{C}$ & -1.146958 & 6.906335 & -13.999912 \\
\hline $\mathrm{C}$ & -3.66083 & -2.120637 & -8.767265 & $\mathrm{H}$ & -1.614041 & 7.860046 & -13.750199 \\
\hline $\mathrm{H}$ & -4.551497 & -2.754845 & -8.856985 & $\mathrm{C}$ & -1.52384 & 5.740527 & -13.324158 \\
\hline $\mathrm{H}$ & -3.264332 & -2.290662 & -7.756853 & $\mathrm{H}$ & -2.280728 & 5.784551 & -12.538971 \\
\hline $\mathrm{C}$ & -4.081127 & -0.64298 & -8.891815 & $\mathrm{C}$ & -0.940196 & 4.518523 & -13.652335 \\
\hline $\mathrm{H}$ & -3.217483 & 0.025792 & -8.764726 & $\mathrm{H}$ & -1.23368 & 3.609505 & -13.125978 \\
\hline $\mathrm{H}$ & -4.518209 & -0.43553 & -9.877927 & $\mathrm{~N}$ & 0.107163 & 0.800692 & -14.584358 \\
\hline $\mathrm{H}$ & -4.830276 & -0.388025 & -8.127549 & $\mathrm{~N}$ & -0.921395 & -0.007567 & -14.783881 \\
\hline $\mathrm{C}$ & -0.654192 & -2.39961 & -8.143844 & $\mathrm{~N}$ & -3.192803 & -1.410054 & -13.896911 \\
\hline $\mathrm{H}$ & 0.415599 & -2.175327 & -8.2268 & $\mathrm{~N}$ & -1.54311 & -2.638945 & -11.820796 \\
\hline $\mathrm{H}$ & -1.113049 & -1.506901 & -7.685421 & $\mathrm{~N}$ & 0.721695 & -2.049532 & -13.405093 \\
\hline $\mathrm{C}$ & -0.852137 & -3.590448 & -7.187505 & $\mathrm{~N}$ & 1.420941 & -2.080495 & -14.530177 \\
\hline $\mathrm{H}$ & -0.390308 & -3.382574 & -6.211146 & $\mathrm{~N}$ & 3.83436 & -0.981023 & -15.878721 \\
\hline $\mathrm{H}$ & -1.917259 & -3.802816 & -7.020847 & $\mathrm{H}$ & 3.505469 & -0.694931 & -14.960673 \\
\hline $\mathrm{H}$ & -0.393013 & -4.502586 & -7.589078 & $\mathrm{~N}$ & 2.654376 & 1.909188 & -15.681232 \\
\hline $\mathrm{C}$ & 1.78379 & -3.175202 & -10.02671 & $\mathrm{H}$ & 2.106293 & 1.195867 & -16.154278 \\
\hline $\mathrm{C}$ & 1.766179 & -4.314428 & -9.206745 & $\mathrm{Pd}$ & -1.210896 & -1.513031 & -13.48309 \\
\hline $\mathrm{H}$ & 0.988052 & -5.063785 & -9.358745 & & & & \\
\hline
\end{tabular}

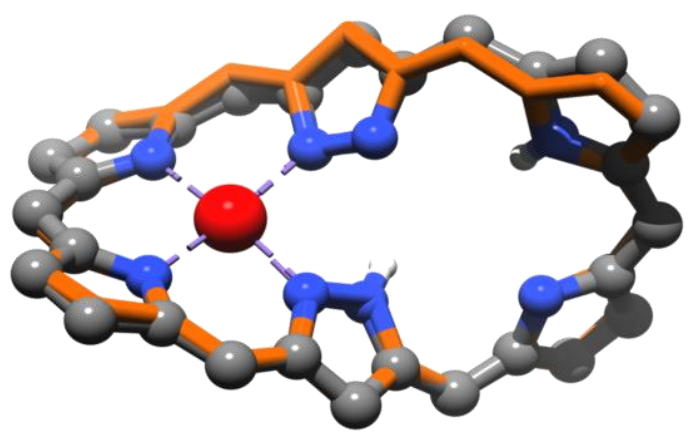

Final Single Point Energy: -4 119.985942986187 Hartrees (no imaginary frequences)

Figure S23: Overlay of the trans-tautomers of $\mathbf{2 7} \mathbf{H}_{2} \mathbf{P d}$ before (ball-and-stick model; palladium in red, carbon in grey, nitrogen in blue, hydrogen in white) and after geometry optimization (wire model; palladium in red, carbon in orange, nitrogen in blue, and hydrogen in white).[93] Peripheral substituents were omitted for clarity. 
Table 13: Optimized CARTESIAn coordinates for the trans-tautomer of $\mathbf{2 7} \mathbf{H}_{2} \mathbf{P d}$.

\begin{tabular}{|c|c|c|c|c|c|c|c|}
\hline & $x$ & $y$ & $Z$ & & $x$ & $y$ & $Z$ \\
\hline $\mathrm{C}$ & -0.13138873 & 1.912204026 & -15.30044562 & $\mathrm{C}$ & 2.41325005 & -5.109777771 & -8.310138099 \\
\hline $\mathrm{C}$ & -1.409522183 & 1.838520889 & -15.91195269 & $\mathrm{H}$ & 2.237075052 & -6.044559534 & -7.775201492 \\
\hline $\mathrm{C}$ & -1.883802675 & 0.537502457 & -15.58412975 & $\mathrm{C}$ & 3.54968927 & -4.347644283 & -8.029620396 \\
\hline $\mathrm{C}$ & -3.090154442 & -0.165010976 & -15.9445746 & $\mathrm{H}$ & 4.258566521 & -4.676904417 & -7.268080671 \\
\hline $\mathrm{C}$ & -3.595281887 & -1.209135989 & -15.1678664 & $\mathrm{C}$ & 3.780660089 & -3.165323897 & -8.740423304 \\
\hline $\mathrm{C}$ & -4.694885985 & -2.135257504 & -15.44999928 & $\mathrm{H}$ & 4.668086185 & -2.565321427 & -8.532013026 \\
\hline $\mathrm{C}$ & -4.984480129 & -2.790152797 & -14.26938208 & $\mathrm{C}$ & 2.88120979 & -2.749144781 & -9.721376618 \\
\hline $\mathrm{C}$ & -4.033689267 & -2.311806444 & -13.26381515 & $\mathrm{H}$ & 3.064116224 & -1.827123215 & -10.27591465 \\
\hline $\mathrm{C}$ & -3.977398357 & -2.627275094 & -11.87797695 & $\mathrm{C}$ & 3.247413797 & -4.569601102 & -12.58526177 \\
\hline $\mathrm{C}$ & -2.772715566 & -2.55533256 & -11.14342874 & $\mathrm{C}$ & 2.705226732 & -5.813200676 & -12.21914807 \\
\hline $\mathrm{C}$ & -2.590891387 & -2.51488724 & -9.689140313 & $\mathrm{H}$ & 1.62499999 & -5.959615767 & -12.2721768 \\
\hline $\mathrm{C}$ & -1.244507411 & -2.654982704 & -9.439601563 & $\mathrm{C}$ & 3.532439926 & -6.861281086 & -11.81464171 \\
\hline $\mathrm{C}$ & -0.573739869 & -2.759030124 & -10.74291871 & $\mathrm{H}$ & 3.092891036 & -7.822603342 & -11.54303573 \\
\hline $\mathrm{C}$ & 0.762375665 & -3.029384975 & -11.03329997 & $\mathrm{C}$ & 4.917815362 & -6.685346073 & -11.76243534 \\
\hline $\mathrm{C}$ & 1.294613911 & -2.832780382 & -12.36614801 & $\mathrm{H}$ & 5.563866774 & -7.505687792 & -11.44555713 \\
\hline $\mathrm{C}$ & 2.387741347 & -3.461198646 & -13.04515443 & $\mathrm{C}$ & 5.46969066 & -5.45261093 & -12.12060282 \\
\hline $\mathrm{C}$ & 2.494767459 & -2.801515772 & -14.28817045 & $\mathrm{H}$ & 6.550395422 & -5.303427143 & -12.07978871 \\
\hline $\mathrm{C}$ & 3.29905699 & -3.101041821 & -15.47411879 & $\mathrm{C}$ & 4.642733954 & -4.405877989 & -12.53036311 \\
\hline $\mathrm{C}$ & 3.81006288 & -2.084958485 & -16.27480261 & $\mathrm{H}$ & 5.07463992 & -3.442116773 & -12.80474974 \\
\hline $\mathrm{C}$ & 4.452109756 & -2.167380962 & -17.60424556 & $\mathrm{C}$ & 3.513266749 & -4.53782708 & -15.77079824 \\
\hline $\mathrm{C}$ & 5.008751734 & -0.932049383 & -17.82673286 & $\mathrm{C}$ & 2.42245772 & -5.426268326 & -15.83178358 \\
\hline $\mathrm{C}$ & 4.633332372 & -0.094564176 & -16.6630589 & $\mathrm{H}$ & 1.414637865 & -5.045181683 & -15.65755412 \\
\hline $\mathrm{C}$ & 5.010144108 & 1.279889612 & -16.42793759 & $\mathrm{C}$ & 2.612577678 & -6.774727186 & -16.13376872 \\
\hline $\mathrm{C}$ & 4.128611283 & 2.170932498 & -15.78707657 & $\mathrm{H}$ & 1.750817509 & -7.441580612 & -16.19436506 \\
\hline $\mathrm{C}$ & 4.365778751 & 3.438796059 & -15.1051605 & $\mathrm{C}$ & 3.900619939 & -7.270575689 & -16.35699899 \\
\hline $\mathrm{C}$ & 3.140878434 & 3.978852767 & -14.75397105 & $\mathrm{H}$ & 4.050847936 & -8.327137743 & -16.58465902 \\
\hline $\mathrm{C}$ & 2.095672507 & 3.050973413 & -15.18583974 & $\mathrm{C}$ & 4.996018317 & -6.405838948 & -16.27532771 \\
\hline $\mathrm{C}$ & 0.71106468 & 3.097987357 & -15.10148094 & $\mathrm{H}$ & 6.006631139 & -6.787986829 & -16.42936225 \\
\hline $\mathrm{C}$ & -2.043713224 & 2.913281747 & -16.6990196 & $\mathrm{C}$ & 4.804602939 & -5.054560358 & -15.98790084 \\
\hline $\mathrm{C}$ & -1.376137342 & 3.455656019 & -17.8134781 & $\mathrm{H}$ & 5.660691754 & -4.382257475 & -15.91382403 \\
\hline $\mathrm{H}$ & -0.406824252 & 3.044751632 & -18.10097759 & $\mathrm{C}$ & 4.30546669 & -3.246677511 & -18.64395733 \\
\hline $\mathrm{C}$ & -1.940179129 & 4.494871614 & -18.55449144 & $\mathrm{H}$ & 4.970946859 & -3.016684564 & -19.48941691 \\
\hline $\mathrm{H}$ & -1.40699498 & 4.894974487 & -19.4188841 & $\mathrm{H}$ & 4.624366535 & -4.224430566 & -18.26101219 \\
\hline $\mathrm{C}$ & -3.184001173 & 5.019366022 & -18.1938117 & $\mathrm{C}$ & 2.860673702 & -3.365301258 & -19.16800181 \\
\hline $\mathrm{H}$ & -3.626532401 & 5.833233267 & -18.7705708 & $\mathrm{H}$ & 2.164954459 & -3.631783411 & -18.36034505 \\
\hline $\mathrm{C}$ & -3.856547571 & 4.49423956 & -17.08679191 & $\mathrm{H}$ & 2.520083277 & -2.415205562 & -19.60534261 \\
\hline $\mathrm{H}$ & -4.824479689 & 4.902555148 & -16.79195805 & $\mathrm{H}$ & 2.792765948 & -4.14328074 & -19.94291755 \\
\hline $\mathrm{C}$ & -3.293285909 & 3.452983655 & -16.34832961 & $\mathrm{C}$ & 5.683843184 & -0.486256268 & -19.09834572 \\
\hline $\mathrm{H}$ & -3.814459781 & 3.060067149 & -15.47446199 & $\mathrm{H}$ & 5.032813192 & -0.748544963 & -19.95046458 \\
\hline $\mathrm{C}$ & -3.813805021 & 0.288728375 & -17.1607823 & $\mathrm{H}$ & 5.766745279 & 0.608816643 & -19.10826832 \\
\hline $\mathrm{C}$ & -3.169197426 & 0.332288634 & -18.40870131 & $\mathrm{C}$ & 7.075857309 & -1.098400745 & -19.33922978 \\
\hline $\mathrm{H}$ & -2.125914595 & 0.021315563 & -18.48021747 & $\mathrm{H}$ & 7.033112112 & -2.196975534 & -19.36560722 \\
\hline
\end{tabular}




\begin{tabular}{|c|c|c|c|c|c|c|c|}
\hline C & -3.851556159 & 0.754712851 & -19.55027407 & $\mathrm{H}$ & 7.490303588 & -0.752024794 & -20.29785287 \\
\hline $\mathrm{H}$ & -3.337065569 & 0.770137786 & -20.51261816 & $\mathrm{H}$ & 7.775148841 & -0.806970877 & -18.54418043 \\
\hline C & -5.185619367 & 1.162762215 & -19. & $\mathrm{C}$ & 218 & 729 & -16.8 \\
\hline $\mathrm{H}$ & -5.714389419 & 1.502479143 & -20.35466106 & $\mathrm{C}$ & 7.502422975 & 0.998781655 & -16.53351691 \\
\hline C & -5.833388492 & 1.144461582 & -18.22444693 & $\mathrm{H}$ & 7.391749192 & 0.090164303 & -15.93882084 \\
\hline $\mathrm{H}$ & -6.870463758 & 1.473928769 & -18.14359941 & $\mathrm{C}$ & 8.77178194 & 1.426525729 & -16.92865342 \\
\hline C & -5.154219763 & 0.709738535 & -17.08619434 & $\mathrm{H}$ & 9.650767024 & 0.843880536 & 08056 \\
\hline $\mathrm{H}$ & -5.657902974 & 0.695523974 & 46801 & $\mathrm{C}$ & 8.919429815 & 2.597559262 & -17.6769042 \\
\hline 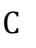 & -5.262859767 & -2.500894956 & -16.79522095 & $\mathrm{H}$ & 9.910438357 & 6303 & 3528 \\
\hline $\mathrm{H}$ & -6.15710284 & -3.122077856 & -16.64029691 & $\mathrm{C}$ & 7.784318076 & 3.338495591 & -18.02594712 \\
\hline $\mathrm{H}$ & -5.602868579 & -1.615172274 & -17.34445391 & $\mathrm{H}$ & 7.887992837 & 4.248615116 & 87423 \\
\hline $\mathrm{C}$ & -4.263318866 & -3.27932543 & -17 & $\mathrm{C}$ & 4823 & 29927 & -1 \\
\hline $\mathrm{H}$ & -3.918953383 & -4.192641668 & -17.16725717 & $\mathrm{H}$ & 5.63420426 & 3.489947807 & -17.90857634 \\
\hline $\mathrm{H}$ & -4.73097 & -3.57 & -18. & $\mathrm{C}$ & 3783 & 649 & 69 \\
\hline $\mathrm{H}$ & -3.378978371 & -2.67008399 & -17.9040793 & $\mathrm{H}$ & 6.406284492 & 4.059985992 & -15.45479074 \\
\hline $\mathrm{C}$ & -5.940638079 & -3.943693603 & -14.11973843 & $\mathrm{H}$ & 5.556470767 & 4.958499274 & -14.20796559 \\
\hline $\mathrm{H}$ & -6.521465709 & -3.852457031 & -13.19414866 & $\mathrm{C}$ & 6.320331757 & 3.049235347 & -13.5 \\
\hline $\mathrm{H}$ & -6.673327775 & -3.908921992 & -14.94029199 & $\mathrm{H}$ & 5.65084798 & 2.956249832 & -12.67296886 \\
\hline $\mathrm{C}$ & -5.236400176 & -5.313853565 & -14.13665444 & $\mathrm{H}$ & 6.511725808 & 2.039699301 & -13.92643616 \\
\hline $\mathrm{H}$ & -5.969517969 & -6.128173368 & -14.04 & $\mathrm{H}$ & 7.2764 & 1578 & -13.1 \\
\hline $\mathrm{H}$ & -4.67984597 & -5.461193336 & -15.07316769 & $\mathrm{C}$ & 2.971824897 & 5.224167557 & 9691 \\
\hline $\mathrm{H}$ & -4.520324541 & -5.403021734 & -13.30718663 & $\mathrm{H}$ & 1.955573889 & 5.264647511 & -13.51306752 \\
\hline C & -5.22429471 & -3.067471715 & 773716 & $\mathrm{H}$ & 798 & 5.147633454 & -13 \\
\hline $\mathrm{C}$ & -6.380647951 & -2.265247026 & -11.22623679 & $\mathrm{C}$ & 3.276929708 & 6.539503435 & 24984 \\
\hline $\mathrm{H}$ & -6.34695048 & -1.308369215 & -11. & $\mathrm{H}$ & 4.30 & 34 & -15 \\
\hline $\mathrm{C}$ & -7.556782325 & -2.677168396 & -10.59713973 & $\mathrm{H}$ & 2.601403367 & 6.680609304 & -15.51556187 \\
\hline $\mathrm{H}$ & -8.439850158 & -2.036071899 & -10.62611561 & $\mathrm{H}$ & 3.151978317 & 7.398699041 & -13.98720538 \\
\hline $\mathrm{C}$ & -7.604815744 & -3.905027436 & -9.929493101 & $\mathrm{C}$ & -0.01478799 & 4.333847621 & -14.71442714 \\
\hline $\mathrm{H}$ & -8.524830784 & -4.228689614 & -9.44055014 & $\mathrm{C}$ & 0.103026177 & 5.531695101 & -15.4405 \\
\hline $\mathrm{C}$ & -6.465021252 & -4.715069667 & -9.893777737 & $\mathrm{H}$ & 0.727642728 & 5.548680642 & -16.33420385 \\
\hline $\mathrm{H}$ & -6.49407064 & -5.677607494 & -9.380296588 & $\mathrm{C}$ & -0.593497066 & 6.677274224 & -15.05163717 \\
\hline $\mathrm{C}$ & -5.287852847 & -4.298778619 & -10.51861714 & $\mathrm{H}$ & -0.496551428 & 7.593416784 & -15.63701507 \\
\hline $\mathrm{H}$ & -4.400137675 & -4.933184901 & -10.48964 & $\mathrm{C}$ & -1.422152834 & 6.649762225 & -13.92724743 \\
\hline $\mathrm{C}$ & -3.618757659 & -2.108726933 & -8.6661 & П & -1.96470224 & 7.546078621 & -13.62276646 \\
\hline $\mathrm{H}$ & -4.530263608 & -2.713315585 & -8.749881916 & $\mathrm{C}$ & -1.56299195 & 5.460329261 & -13.20387713 \\
\hline $\mathrm{H}$ & -3.215841449 & -2.29892806 & -7.662258969 & $\mathrm{H}$ & -2.209773774 & 5.427208267 & -12.325223 \\
\hline $\mathrm{C}$ & -3.992001784 & -0.617264892 & -8.775631458 & $\mathrm{C}$ & -0.878666695 & 4.312114471 & -13.60082766 \\
\hline 11 & -3.106540208 & 0.022893374 & -8.652799442 & $\mathrm{H}$ & -0.989776002 & 3.385367227 & -13.03540618 \\
\hline $\mathrm{H}$ & -4.434356403 & -0.387092213 & -9.754307617 & $\mathrm{~N}$ & 0.165911314 & 0.723407378 & -14.69170013 \\
\hline $\mathrm{H}$ & -4.72332178 & -0.345398034 & -8.000307285 & $\mathrm{~N}$ & -0.896561727 & -0.07570874 & -14.83164768 \\
\hline $\mathrm{C}$ & -0.616193253 & -2.522688219 & -8.073781223 & $\mathrm{~N}$ & -3.162230581 & -1.43546876 & -13.85296369 \\
\hline $\mathrm{H}$ & 0.464815685 & -2.365364766 & -8.168547748 & $\mathrm{~N}$ & -1.537942799 & -2.597102445 & -11.74571963 \\
\hline$\Pi$ & -1.013185141 & -1.601601066 & -7.612928015 & $\mathrm{~N}$ & 0.777615499 & -1.858073571 & -13.17429897 \\
\hline $\mathrm{C}$ & -0.876631984 & -3.695502838 & -7.110404629 & $\mathrm{~N}$ & 1.526647872 & -1.843407442 & -14.2915018 \\
\hline $\mathrm{H}$ & -0.39606475 & -3.50803743 & -6.139285254 & $\mathrm{~N}$ & 3.863895108 & -0.777464458 & -15.7995145 \\
\hline
\end{tabular}




$\begin{array}{rrrrrrrr}\mathrm{H} & -1.950418025 & -3.84568234 & -6.93283529 & \mathrm{~N} & 2.770468201 & 1.974156982 & -15.74385377 \\ \mathrm{H} & -0.472035654 & -4.633735117 & -7.509053688 & \mathrm{H} & 2.32780942 & 1.108753788 & -16.03519721 \\ \mathrm{C} & 1.717441003 & -3.49265851 & -9.993764232 & \mathrm{Pd} & -1.203302501 & -1.463804899 & -13.3942227 \\ \mathrm{C} & 1.50555434 & -4.685169252 & -9.281714059 & \mathrm{H} & 1.428871192 & -1.029513014 & -14.9050232 \\ \mathrm{H} & 0.628508562 & -5.290895914 & -9.51496074 & & & & \end{array}$

\subsubsection{Spin Density Distributions}

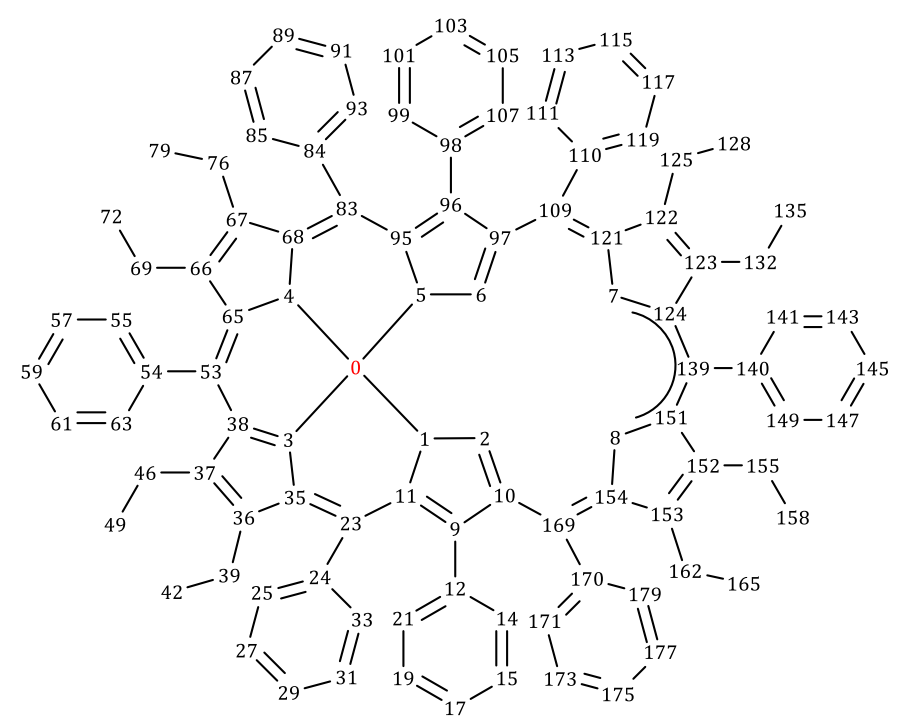

Table 14: Selected calculated MuLLIKEN atomic charges and spin populations ( $> \pm 0.1 \%$ ) for $\mathbf{2 7} \mathbf{H}_{2} \mathbf{P d}^{-}$.

\begin{tabular}{llrrrlrr}
\hline$\#$ & & atomic charge & spin population & \multicolumn{1}{l}{$\#$} & & atomic charge & spin population \\
\hline 0 & $\mathrm{Pd}:$ & 0.063056 & -0.004051 & 117 & $\mathrm{C}:$ & -0.20614 & -0.0166 \\
1 & $\mathrm{~N}:$ & -0.051993 & 0.004606 & 119 & $\mathrm{C}:$ & -0.318743 & 0.034948 \\
2 & $\mathrm{~N}:$ & -0.233197 & 0.027021 & 120 & $\mathrm{H}:$ & 0.239365 & -0.001461 \\
4 & $\mathrm{~N}:$ & -0.32384 & -0.001166 & 121 & $\mathrm{C}:$ & 0.234413 & -0.09164 \\
5 & $\mathrm{~N}:$ & -0.029063 & 0.004588 & 122 & $\mathrm{C}:$ & -0.090671 & 0.118891 \\
6 & $\mathrm{~N}:$ & -0.246802 & 0.021187 & 123 & $\mathrm{C}:$ & -0.058208 & -0.074004 \\
7 & $\mathrm{~N}:$ & -0.148738 & 0.023645 & 124 & $\mathrm{C}:$ & 0.146789 & 0.155059 \\
8 & $\mathrm{~N}:$ & -0.146914 & 0.038209 & 125 & $\mathrm{C}:$ & -0.267661 & -0.006838 \\
9 & $\mathrm{C}:$ & 0.151478 & 0.052943 & 126 & $\mathrm{H}:$ & 0.134669 & 0.002412 \\
10 & $\mathrm{C}:$ & 0.058269 & -0.041874 & 128 & $\mathrm{C}:$ & -0.444367 & 0.004132 \\
11 & $\mathrm{C}:$ & 0.037117 & -0.007967 & 130 & $\mathrm{H}:$ & 0.107832 & 0.001038 \\
12 & $\mathrm{C}:$ & 0.05797 & -0.004577 & 132 & $\mathrm{C}:$ & -0.279243 & 0.004336 \\
13 & $\mathrm{C}:$ & -0.389218 & 0.001201 & 134 & $\mathrm{H}:$ & 0.166734 & -0.001548 \\
17 & $\mathrm{C}:$ & -0.221164 & 0.002068 & 135 & $\mathrm{C}:$ & -0.461067 & -0.00302 \\
21 & $\mathrm{C}:$ & -0.233641 & 0.00414 & 139 & $\mathrm{C}:$ & -0.002038 & -0.156617 \\
23 & $\mathrm{C}:$ & -0.021409 & 0.013035 & 140 & $\mathrm{C}:$ & 0.049864 & 0.013174 \\
35 & $\mathrm{C}:$ & 0.319217 & -0.001004 & 141 & $\mathrm{C}:$ & -0.39035 & -0.012923
\end{tabular}




\begin{tabular}{|c|c|c|c|c|c|c|c|}
\hline 36 & $\mathrm{C}:$ & -0.050831 & 0.006327 & 143 & $\mathrm{C}:$ & -0.134653 & 0.010273 \\
\hline 37 & $C:$ & -0.103298 & -0.001619 & 145 & $\mathrm{C}:$ & -0.301652 & -0.018733 \\
\hline 38 & C: & 0.366691 & 0.011573 & 146 & $\mathrm{H}:$ & 0.247411 & 0.001321 \\
\hline 53 & C : & -0.207543 & -0.010356 & 147 & $\mathrm{C}:$ & -0.215172 & 0.010921 \\
\hline 65 & $\mathrm{C}:$ & 0.331178 & 0.010625 & 149 & $\mathrm{C}:$ & -0.327932 & -0.014755 \\
\hline 66 & $C:$ & -0.06159 & -0.002477 & 151 & $\mathrm{C}:$ & 0.091911 & 0.198228 \\
\hline 67 & $C:$ & -0.095242 & 0.006194 & 152 & $\mathrm{C}:$ & 0.043564 & -0.072239 \\
\hline 68 & $\mathrm{C}:$ & 0.381494 & -0.001499 & 153 & $\mathrm{C}:$ & -0.244128 & 0.157125 \\
\hline 83 & $\mathrm{C}:$ & -0.079371 & 0.009353 & 154 & $\mathrm{C}:$ & 0.356266 & -0.094404 \\
\hline 95 & $C:$ & 0.082692 & -0.005599 & 180 & $\mathrm{H}:$ & 0.247163 & -0.001601 \\
\hline 96 & $\mathrm{C}:$ & 0.13005 & 0.037836 & 181 & $\mathrm{H}:$ & 0.179755 & -0.001009 \\
\hline 97 & $C:$ & 0.023053 & -0.032066 & 182 & $\mathrm{H}:$ & 0.157667 & -0.001117 \\
\hline 98 & $\mathrm{C}:$ & 0.131525 & -0.003597 & 171 & $\mathrm{C}:$ & -0.381063 & 0.05091 \\
\hline 99 & C: & -0.288351 & 0.001318 & 172 & $\mathrm{H}:$ & 0.250209 & -0.0017 \\
\hline 109 & $C:$ & -0.006738 & 0.275982 & 173 & $\mathrm{C}:$ & -0.206424 & -0.023279 \\
\hline 110 & $C:$ & -0.013681 & -0.033923 & 175 & $\mathrm{C}:$ & -0.185086 & 0.053819 \\
\hline 111 & $\mathrm{C}:$ & -0.266372 & 0.034693 & 176 & $\mathrm{H}:$ & 0.170402 & -0.002882 \\
\hline 113 & $\mathrm{C}:$ & -0.311141 & -0.015962 & 177 & $\mathrm{C}:$ & -0.259986 & -0.023552 \\
\hline 115 & $C:$ & -0.203979 & 0.036718 & 179 & $\mathrm{C}:$ & -0.368349 & 0.047366 \\
\hline 116 & $\mathrm{H}:$ & 0.184885 & -0.002275 & & & & \\
\hline
\end{tabular}

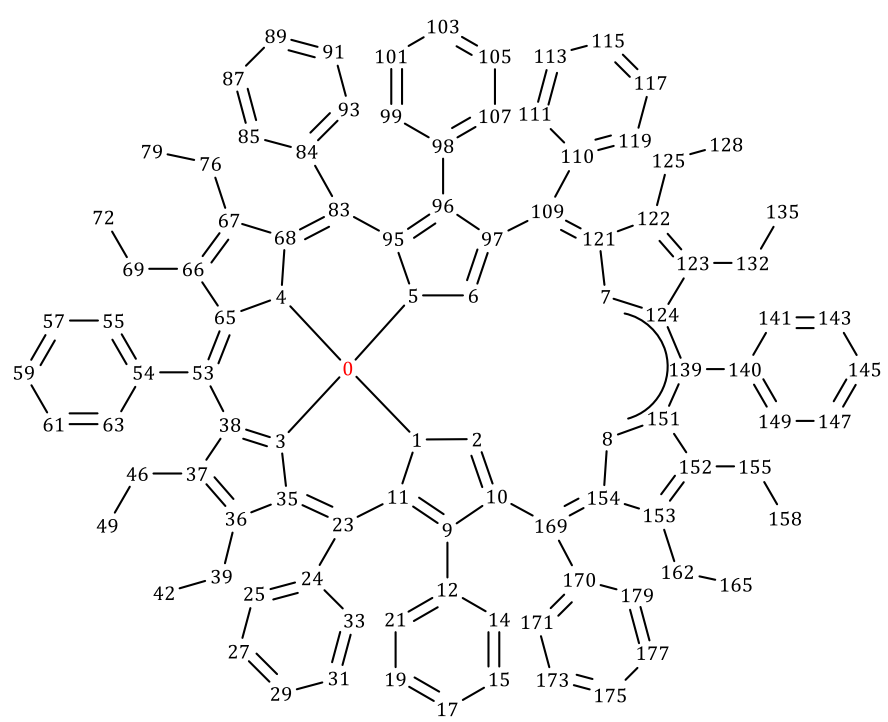

Table 15: Selected calculated MuLLIKEN atomic charges and spin populations ( $> \pm 0.1 \%$ ) for $\mathbf{2 7} \mathbf{H}_{2} \mathbf{P d}^{+}$.

\begin{tabular}{rrrrrlrr}
\hline$\#$ & & atomic charge & spin population & \multicolumn{1}{l}{$\#$} & & atomic charge & spin population \\
\hline 0 & $\mathrm{Pd}:$ & 0.104996 & 0.021949 & 55 & $\mathrm{C}:$ & -0.441905 & 0.021805 \\
2 & $\mathrm{~N}:$ & -0.222642 & 0.089956 & 57 & $\mathrm{C}:$ & -0.151343 & -0.007983 \\
3 & $\mathrm{~N}:$ & -0.275436 & 0.126597 & 59 & $\mathrm{C}:$ & -0.211424 & 0.018877 \\
4 & $\mathrm{~N}:$ & -0.297546 & 0.140231 & 61 & $\mathrm{C}:$ & -0.28335 & -0.008082 \\
6 & $\mathrm{~N}:$ & -0.233134 & 0.093014 & 63 & $\mathrm{C}:$ & -0.292804 & 0.021654
\end{tabular}




\begin{tabular}{llrrrlrr}
\hline 7 & $\mathrm{~N}:$ & -0.144868 & 0.002921 & 64 & $\mathrm{H}:$ & 0.219979 & -0.00108 \\
8 & $\mathrm{~N}:$ & -0.135954 & 0.002405 & 65 & $\mathrm{C}:$ & 0.346763 & -0.097532 \\
9 & $\mathrm{C}:$ & 0.190135 & 0.075398 & 66 & $\mathrm{C}:$ & -0.049194 & 0.01709 \\
10 & $\mathrm{C}:$ & 0.072763 & -0.022584 & 67 & $\mathrm{C}:$ & -0.087255 & -0.024144 \\
11 & $\mathrm{C}:$ & 0.061723 & -0.017007 & 68 & $\mathrm{C}:$ & 0.401296 & -0.035022 \\
12 & $\mathrm{C}:$ & 0.060027 & -0.005921 & 76 & $\mathrm{C}:$ & -0.247049 & 0.002516 \\
13 & $\mathrm{C}:$ & -0.399232 & 0.004758 & 79 & $\mathrm{C}:$ & -0.373587 & -0.001085 \\
15 & $\mathrm{C}:$ & -0.176024 & -0.001641 & 83 & $\mathrm{C}:$ & -0.066014 & 0.18753 \\
17 & $\mathrm{C}:$ & -0.20975 & 0.003776 & 84 & $\mathrm{C}:$ & 0.110346 & -0.017738 \\
19 & $\mathrm{C}:$ & -0.284939 & -0.001476 & 85 & $\mathrm{C}:$ & -0.428362 & 0.011466 \\
21 & $\mathrm{C}:$ & -0.252024 & 0.004751 & 87 & $\mathrm{C}:$ & -0.172415 & -0.003229 \\
23 & $\mathrm{C}:$ & -0.005231 & 0.173986 & 89 & $\mathrm{C}:$ & -0.212411 & 0.010432 \\
24 & $\mathrm{C}:$ & 0.026277 & -0.014151 & 91 & $\mathrm{C}:$ & -0.213042 & -0.004761 \\
25 & $\mathrm{C}:$ & -0.363892 & 0.011996 & 93 & $\mathrm{C}:$ & -0.410981 & 0.012497 \\
26 & $\mathrm{H}:$ & 0.244673 & -0.001455 & 95 & $\mathrm{C}:$ & 0.108516 & -0.014334 \\
27 & $\mathrm{C}:$ & -0.244722 & -0.003069 & 96 & $\mathrm{C}:$ & 0.168521 & 0.071974 \\
29 & $\mathrm{C}:$ & -0.213721 & 0.009964 & 97 & $\mathrm{C}:$ & 0.029986 & -0.022507 \\
31 & $\mathrm{C}:$ & -0.222658 & -0.005032 & 98 & $\mathrm{C}:$ & 0.128578 & -0.005363 \\
33 & $\mathrm{C}:$ & -0.292042 & 0.011864 & 99 & $\mathrm{C}:$ & -0.305852 & 0.002635 \\
35 & $\mathrm{C}:$ & 0.33674 & -0.038583 & 107 & $\mathrm{C}:$ & -0.286684 & 0.0025 \\
36 & $\mathrm{C}:$ & -0.044694 & -0.021499 & 109 & $\mathrm{C}:$ & 0.038447 & 0.004394 \\
37 & $\mathrm{C}:$ & -0.085597 & 0.016352 & 121 & $\mathrm{C}:$ & 0.242276 & -0.003246 \\
38 & $\mathrm{C}:$ & 0.379748 & -0.094498 & 124 & $\mathrm{C}:$ & 0.189932 & -0.003293 \\
39 & $\mathrm{C}:$ & -0.291656 & 0.003346 & 139 & $\mathrm{C}:$ & -0.009878 & 0.006553 \\
42 & $\mathrm{C}:$ & -0.47865 & -0.001545 & 151 & $\mathrm{C}:$ & 0.143544 & -0.003414 \\
49 & $\mathrm{C}:$ & -0.430261 & 0.001022 & 154 & $\mathrm{C}:$ & 0.368479 & -0.0038 \\
53 & $\mathrm{C}:$ & -0.154378 & 0.332989 & 169 & $\mathrm{C}:$ & -0.023535 & 0.004028 \\
54 & $\mathrm{C}:$ & 0.037107 & -0.032098 & & & & \\
\hline & & & & & & & \\
\hline
\end{tabular}




\section{REFERENCES}

[1] L. H. Gade, J. Lewis, Koordinationschemie; Wiley-VCH, Weinheim, 1998.

[2] a) R. A. Khatypov, A. Y. Khmelnitskiy, M. M. Leonova, L. G. Vasilieva, V. A. Shuvalov, Photosyn. Res. 2008, 98, 81-93; b) B. K. Biswal, M. Vijayan, Acta Crystallogr, Sect. D: Biol. Crystallogr. 2002, 58, 1155-1161; c) S. E. Phillips, J. Mol. Biol. 1980, 142, 531-554; d) T. Takano, R. E. Dickerson, Proc. Natl. Acad. Sci. U.S.A. 1980, 77, 6371-6375.

[3] W. Kaim, B. Schwederski, Bioanorganische Chemie: Zur Funktion chemischer Elemente in Lebensprozessen; Vieweg+Teubner, Wiesbaden, 2010.

[4] A. Messerschmidt, R. Huber, T. Poulas, K. Wieghardt, M. Cygler, W. Bode, Eds, Handbook of Metalloproteins; John Wiley \& Sons, Ltd, Chichester, 2006.

[5] R. Raag, T. L. Poulos, R. Raag, T. L. Poulos, Biochemistry 1991, 30, 2674-2684.

[6] S. Saito, A. Osuka, Angew. Chem. 2011, 123, 4432-4464; Angew. Chem. Int. Ed. 2011, 50, 4342-4373.

[7] F. P. Guengerich, Chem. Res. Toxicol. 2008, 21, 70-83.

[8] T. Omura, R. Sato, J. Biol. Chem. 1964, 239, 2370-2378.

[9] M. Sono, M. P. Roach, E. D. Coulter, J. H. Dawson, Chem. Rev. 1996, 96, 2841-2888.

[10] D. R. Nelson, Hum. Genomics 2009, 4, 1-7.

[11] J. Rittle, M. T. Green, Science 2010, 330, 933-937.

[12] M. J. Gunter, P. Turner, Coord. Chem. Rev. 1991, 108, 115-161.

[13] J. H. Dawson, M. Sono, Chem. Rev. 1987, 87, 1255-1276.

[14] J. A. McCleverty, T. J. Meyer, Comprehensive Coordination Chemistry II: From Biology to Nanotechnology; Elsevier, Amsterdam, 2004.

[15] B. Meunier, J. Bernadou, Top. Catal. 2002, 21, 47-54.

[16] T. L. Poulos, R. Raag, FASEB J. 1992, 6, 674-679.

[17] E. Mesbahi, N. Safari, M. Gheidi, J. Porphyrins Phthalocyanines 2014, 18, 354-365.

[18] a) J. T. Groves, T. E. Nemo, R. S. Myers, J. Am. Chem. Soc. 1979, 101, 1032-1033; b) C. K. Chang, M.-S. Kuo, J. Am. Chem. Soc. 1979, 101, 3413-3415; c) C. L. Hill, B. C. Schardt, J. Am. Chem. Soc. 1980, 102, 6374-6375; d) J. T. Groves, W. J. Kruper, R. C. Haushalter, J. Am. Chem. Soc. 1980, 102, 6375-6377; e) E. Guilmet, B. Meunier, Tetrahedron Lett. 1980, 21, 4449-4450; f) J. T. Groves, R. C. Haushalter, M. Nakamura, T. E. Nemo, B. J. Evans, J. Am. Chem. Soc. 1981, 103, 2884-2886.

[19] a) M. H. Gelb, W. A. Toscano, S. G. Sligar, Proc. Natl. Acad. Sci. U.S.A. 1982, 79, 5758-5762;

b) N. Hessenauer-Ilicheva, A. Franke, M. Wolak, T. Higuchi, R. van Eldik, Chem. Eur. J. 2009, 15, 12447-12459.

[20] M. F. Powell, E. F. Pai, T. C. Bruice, J. Am. Chem. Soc. 1984, 106, 3277-3285.

[21] a) F. Lanucara, M. E. Crestoni, Chem. Eur. J. 2011, 17, 12092-12100; b) W. J. Song, M. S. Seo, S. DeBeer George, T. Ohta, R. Song, M.-J. Kang, T. Tosha, T. Kitagawa, E. I. Solomon, W. Nam, J. Am. Chem. Soc. 2007, 129, 1268-1277; c) J. T. Groves, J. Lee, S. S. Marla, J. Am. Chem. Soc. 1997, 119, 6269-6273.

[22] a) Z. Pan, M. Newcomb, Inorg. Chem. 2007, 46, 6767-6774; b) N. Hessenauer-Ilicheva, A. Franke, D. Meyer, W.-D. Woggon, R. van Eldik, Chem. Eur. J. 2009, 15, 2941-2959.

[23] J. T. Groves, T. Takahashi, J. Am. Chem. Soc. 1983, 105, 2073-2074.

[24] L. A. Bottomley, F. L. Neely, J. N. Gorce, Inorg. Chem. 1988, 27, 1300-1303.

[25] J. W. Buchler, C. Dreher, Z. Naturforsch, B: J. Chem. Sci. 1984, 39, 222-230.

[26] J. T. Groves, T. Takahashi, W. M. Butler, Inorg. Chem. 1983, 22, 884-887. 
[27] G. Izzet, E. Ishow, J. Delaire, C. Afonso, J.-C. Tabet, A. Proust, Inorg. Chem. 2009, 48, 11865-11870.

[28] J. C. Barona-Castano, C. C. Carmona-Vargas, T. J. Brocksom, K. T. de Oliveira, Molecules 2016, 21, 310.

[29] J. L. Sessler, D. Seidel, Angew. Chem. 2003, 115, 5292-5333; Angew. Chem. Int. Ed. 2003, $42,5134-5175$.

[30] A. Osuka, S. Saito, Chem. Commun. 2011, 47, 4330-4339.

[31] H. Furuta, H. Maeda, A. Osuka, Chem. Commun. 2002, 1795-1804.

[32] M. Toganoh, H. Furuta, Chem. Commun. 2012, 48, 937.

[33] a) A. M. Young, A. L. Von Ruden, T. D. Lash, Org. Biomol. Chem. 2011, 9, 6293; b) C. G. Claessens, D. Gonzalez-Rodriguez, M. S. Rodriguez-Morgade, A. Medina, T. Torres, Chem. Rev. 2014, 114, 2192-2277; c) T. Tanaka, A. Osuka, Chem. Soc. Rev. 2015, 44, 943-969; d) T. Tanaka, A. Osuka, Chem. Rev. 2016; DOI: 10.1021/acs.chemrev.6b00371.

[34] Y. Tanaka, J.-Y. Shin, A. Osuka, Eur. J. Org. Chem. 2008, 1341-1349.

[35] a) H. Mori, T. Tanaka, S. Lee, J. M. Lim, D. Kim, A. Osuka, J. Am. Chem. Soc. 2015, 137, 2097-2106; b) M. Kitano, S.-y. Hayashi, T. Tanaka, H. Yorimitsu, N. Aratani, A. Osuka, Angew. Chem. 2012, 124, 5691-5695; Angew. Chem. Int. Ed. 2012, 51, 5593-5597.

[36] T. K. Chandrashekar, S. Venkatraman, Acc. Chem. Res. 2003, 36, 676-691.

[37] A. Jasat, D. Dolphin, Chem. Rev. 1997, 97, 2267-2340.

[38] J.-Y. Shin, K. S. Kim, M.-C. Yoon, J. M. Lim, Z. S. Yoon, A. Osuka, D. Kim, Chem. Soc. Rev. 2010, 39, 2751-2767.

[39] J. L. Sessler, E. Tomat, Acc. Chem. Res. 2007, 40, 371-379.

[40] H. Mori, M. Suzuki, W. Kim, J. M. Lim, D. Kim, A. Osuka, Chem. Sci. 2015, 6, 1696-1700.

[41] K. S. Kim, Z. S. Yoon, A. B. Ricks, J.-Y. Shin, S. Mori, J. Sankar, S. Saito, Y. M. Jung, M. R. Wasielewski, A. Osuka, D. Kim, J. Phys. Chem. A 2009, 113, 4498-4506.

[42] M. Stępień, L. Latos-Grażyński, N. Sprutta, P. Chwalisz, L. Szterenberg, Angew. Chem. 2007, 119, 8015-8019; Angew. Chem. Int. Ed. 2007, 46, 7716.

[43] R. Herges, Chem. Rev. 2006, 106, 4820-4842.

[44] E. Heilbronner, Tetrahedron Lett. 1964, 5, 1923-1928.

[45] M. Stępień, N. Sprutta, L. Latos-Grażyński, Angew. Chem. 2011, 123, 4376-4430; Angew. Chem. Int. Ed. 2011, 50, 4288-4340.

[46] M.-C. Yoon, P. Kim, H. Yoo, S. Shimizu, T. Koide, S. Tokuji, S. Saito, A. Osuka, D. Kim, J. Phys. Chem. B 2011, 115, 14928-14937.

[47] A. Chaudhary, A. Srinivasan, T. K. Chandrashekar in The Porphyrin Handbook, Vol. 32; (Eds. K. M. Kadish, K. M. Smith, R. Guilard), Academic Press, San Diego, 2000, pp. 271-366.

[48] J. M. Lintuluoto, K. Nakayama, J. Setsune, Chem. Commun. 2006, 3492-3494.

[49] J. Sankar, S. Mori, S. Saito, H. Rath, M. Suzuki, Y. Inokuma, H. Shinokubo, K. S. Kim, Z. S. Yoon, J.-Y. Shin, J. M. Lim, Y. Matsuzaki, O. Matsushita, A. Muranaka, N. Kobayashi, D. Kim, A. Osuka, J. Am. Chem. Soc. 2008, 130, 13568-13579.

[50] S.-i. Ishida, T. Higashino, S. Mori, H. Mori, N. Aratani, T. Tanaka, J. M. Lim, D. Kim, A. Osuka, Angew. Chem. 2014, 126, 3495-3499; Angew. Chem. Int. Ed. 2014, 53, 3427-3431.

[51] J.-Y. Shin, H. Furuta, K. Yoza, S. Igarashi, A. Osuka, J. Am. Chem. Soc. 2001, 123, 71907191.

[52] M. Suzuki, A. Osuka, Chem. Eur. J. 2007, 13, 196-202.

[53] W. Cha, J. M. Lim, M.-C. Yoon, Y. M. Sung, B. S. Lee, S. Katsumata, M. Suzuki, H. Mori, Y. Ikawa, H. Furuta, A. Osuka, D. Kim, Chem. Eur. J. 2012, 18, 15838-15844. 
[54] T. Koide, K. Youfu, S. Saito, A. Osuka, Chem. Commun. 2009, 6047-6049.

[55] M. Suzuki, R. Taniguchi, A. Osuka, Chem. Commun. 2004, 2682-2683.

[56] S. Tokuji, J.-Y. Shin, K. S. Kim, J. M. Lim, K. Youfu, S. Saito, D. Kim, A. Osuka, J. Am. Chem. Soc. 2009, 131, 7240-7241.

[57] M. Inoue, K. S. Kim, M. Suzuki, J. M. Lim, J.-Y. Shin, D. Kim, A. Osuka, Angew. Chem. 2009, 121, 6815-6818; Angew. Chem. Int. Ed. 2009, 48, 6687-6690.

[58] K. Honjo, H. Mori, A. Osuka, Asian J. Org. Chem. 2016, 5, 196-200.

[59] M. Suzuki, A. Osuka, J. Am. Chem. Soc. 2007, 129, 464-465.

[60] M. Bröring, J. Jendrny, L. Zander, H. Schmickler, J. Lex, Y. Wu, M. Nendel, J. Chen, D. A. Plattner, K. N. Houk, E. Vogel, Angew. Chem. 1995, 107, 2709-2711; Angew. Chem. Int. Ed. 1995, 34, 2515-2517.

[61] W. Cha, T. Soya, T. Tanaka, H. Mori, Y. Hong, S. Lee, K. H. Park, A. Osuka, D. Kim, Chem. Commun. 2016, 52, 6076-6078.

[62] J. M. Lim, J.-Y. Shin, Y. Tanaka, S. Saito, A. Osuka, D. Kim, J. Am. Chem. Soc. 2010, 132, 3105-3114.

[63] M. Mori, T. Okawa, N. Iizuna, K. Nakayama, J. M. Lintuluoto, J. Setsune, J. Org. Chem. 2009, $74,3579-3582$.

[64] J.-P. Gisselbrecht, J. Bley-Escrich, M. Gross, L. Zander, M. Michels, E. Vogel, J. Electroanal. Chem. 1999, 469, 170-175.

[65] Z. S. Yoon, A. Osuka, D. Kim, Nat. Chem. 2009, 1, 113-122.

[66] H. Mori, Y. M. Sung, B. S. Lee, D. Kim, A. Osuka, Angew. Chem. 2012, 124, 12627-12631; Angew. Chem. Int. Ed. 2012, 51, 12459-12463.

[67] Y. Kamimura, S. Shimizu, A. Osuka, Chem. Eur. J. 2007, 13, 1620-1628.

[68] J. Bley-Escrich, J.-P. Gisselbrecht, E. Vogel, M. Gross, Eur. J. Inorg. Chem. 2002, 28292837.

[69] J. L. Sessler, E. Tomat, V. M. Lynch, Chem. Commun. 2006, 4486-4488.

[70] a) A. Werner, M. Michels, L. Zander, J. Lex, E. Vogel, Angew. Chem. 1999, 111, 3866-3870; Angew. Chem. Int. Ed. 1999, 38, 3650-3653; b) J. L. Sessler, A. Gebauer, S. J. Weghorn in The Porphyrin Handbook, Vol. 2; (Eds. K. M. Kadish, K. M. Smith, R. Guilard), Academic Press, San Diego, 2000, pp. 55-124.

[71] H. Rath, S. Tokuji, N. Aratani, K. Furukawa, J. M. Lim, D. Kim, H. Shinokubo, A. Osuka, Angew. Chem. 2010, 122, 1531-1533; Angew. Chem. Int. Ed. 2010, 49, 1489-1491.

[72] T. Yoneda, Y. M. Sung, J. M. Lim, D. Kim, A. Osuka, Angew. Chem. 2014, 126, 1338513389; Angew. Chem. Int. Ed. 2014, 53, 13169-13173.

[73] G. Hohlneicher, D. Bremm, J. Wytko, J. Bley-Escrich, J.-P. Gisselbrecht, M. Gross, M. Michels, J. Lex, E. Vogel, Chem. Eur. J. 2003, 9, 5636-5642.

[74] E. Vogel, M. Michels, L. Zander, J. Lex, N. S. Tuzun, K. N. Houk, Angew. Chem. 2003, 115, 2964-2969; Angew. Chem. Int. Ed. 2003, 42, 2857-2862.

[75] L. K. Frensch, K. Pröpper, M. John, S. Demeshko, C. Brückner, F. Meyer, Angew. Chem. 2011, 123, 1456-1460; Angew. Chem. Int. Ed. 2011, 50, 1420-1424.

[76] L. K. Blusch, Y. Hemberger, K. Pröpper, B. Dittrich, F. Witterauf, M. John, G. Bringmann, C. Brückner, F. Meyer, Chem. Eur. J. 2013, 19, 5868-5880.

[77] E. J. Lind; Ph.D. Thesis, Michigan State University, 1987.

[78] A. Sachse, L. Penkova, G. Noël, S. Dechert, O. Varzatskii, I. O. Fritsky, F. Meyer, Synthesis 2008, 800-806.

[79] Unpublished results. 
[80] S. Katsiaouni, S. Dechert, R. P. Briñas, C. Brückner, F. Meyer, Chem. Eur. J. 2008, 14, 48234835.

[81] O. Mitevski, S. Dechert, C. Brückner, F. Meyer, Eur. J. Inorg. Chem. 2016, 4814-4819.

[82] L. K. Blusch, O. Mitevski, V. Martin-Diaconescu, K. Pröpper, S. DeBeer, S. Dechert, F. Meyer, Inorg. Chem. 2014, 53, 7876-7885.

[83] L. K. Blusch, K. E. Craigo, V. Martin-Diaconescu, A. B. McQuarters, E. Bill, S. Dechert, S. DeBeer, N. Lehnert, F. Meyer, J. Am. Chem. Soc. 2013, 135, 13892-13899.

[84] L. K. Blusch, The Siamese-Twin Porphyrin and its copper and nickel complexes: A noninnocent twist; Springer International Publishing, Switzerland, 2013.

[85] O. Mitevski; PhD Thesis, Georg-August-University, 2016.

[86] Y. Ling, Y. Zhang, J. Am. Chem. Soc. 2009, 131, 6386-6388.

[87] a) V. Schünemann, M. Gerdan, A. X. Trautwein, N. Haoudi, D. Mandon, J. Fischer, R. Weiss, A. Tabard, R. Guilard, Angew. Chem. 1999, 111, 3376-3379; Angew. Chem. Int. Ed. 1999, 38, 3181-3183; b) R.-J. Cheng, P.-Y. Chen, P.-R. Gau, C.-C. Chen, S.-M. Peng, J. Am. Chem. Soc. 1997, 119, 2563-2569.

[88] A. Vogel, S. Dechert, M. John, C. Brückner, F. Meyer, Chem. Eur. J. 2016, 22, 2307-2316.

[89] D. R. Buckle, S. J. Collier, M. D. McLaws in Encyclopedia of Reagents for Organic Synthesis; (Ed. L. A. Paquette), John Wiley \& Sons, Ltd, Chichester, UK, 2001.

[90] S. Shimizu, J.-Y. Shin, H. Furuta, R. Ismael, A. Osuka, Angew. Chem. 2003, 115, 82-86; Angew. Chem. Int. Ed. 2003, 42, 78-82.

[91] W. R. Scheidt, I. Turowska-Tyrk, Inorg. Chem. 1994, 33, 1314-1318.

[92] M. O. Senge in The Porphyrin Handbook, Vol. 10; (Eds. K. M. Kadish, K. M. Smith, R. Guilard), Academic Press, San Diego, 2000, pp. 1-218.

[93] E. F. Pettersen, T. D. Goddard, C. C. Huang, G. S. Couch, D. M. Greenblatt, E. C. Meng, T. E. Ferrin, J. Comput. Chem. 2004, 25, 1605-1612.

[94] C. Janiak, J. Chem. Soc, Dalton Trans. 2000, 3885-3896.

[95] a) J. R. McCarthy, M. A. Hyland, C. Brückner, Org. Biomol. Chem. 2004, 2, 1484-1491; b) L. P. Samankumara, S. J. Dorazio, J. Akhigbe, R. Li, A. Nimthong-Roldán, M. Zeller, C. Brückner, Chem. Eur. J. 2015, 21, 11118-11128.

[96] A. Gossauer, N. Engel, J. Photochem. Photobiol, B 1996, 32, 141-151.

[97] S. Saito, K. Furukawa, A. Osuka, J. Am. Chem. Soc. 2010, 132, 2128-2129.

[98] P. Morosini, M. Scherer, S. Meyer, V. Lynch, J. L. Sessler, J. Org. Chem. 1997, 62, 88488853.

[99] K. T. Potts, Chem. Rev. 1961, 61, 87-127.

[100] J. Olguín, M. Kalisz, R. Clérac, S. Brooker, Inorg. Chem. 2012, 51, 5058-5069.

[101] a) A. Naik, J. Marchand-Brynaert, Y. Garcia, Synthesis 2008, 149-154; b) N. V. Ivanova, S. I. Sviridov, S. V. Shorshnev, A. E. Stepanov, Synthesis 2006, 156-160.

[102] a) J. Bergman, T. Janosik in Modern Heterocyclic Chemistry; (Eds. J. Alvarez-Builla, J. J. Vaquero, J. Barluenga), Wiley-VCH, Weinheim, 2011, pp. 269-375; b) D. A. Shirley, B. H. Gross, P. A. Roussel, J. Org. Chem. 1955, 20, 225-231.

[103] Verordnung des Rates vom 13. Dezember 1990 über Maßnahmen gegen die Abzweigung bestimmter Stoffe zur unerlaubten Herstellung von Suchtstoffen und psychotropen Substanzen: EWG Nr. 3677/90, 1990.

[104] E. C. Y. Woon, P. T. Sunderland, H. A. Paine, M. D. Lloyd, A. S. Thompson, M. D. Threadgill, Bioorg. Med. Chem. 2013, 21, 5218-5227. 
[105] B. Meunier, A. Robert, G. Pratviel, J. Bernadou in The Porphyrin Handbook, Vol. 4; (Eds. K. M. Kadish, K. M. Smith, R. Guilard), Academic Press, San Diego, 2000, pp. 119-187.

[106] A. Herter; Master Thesis, Georg-August-University, 2012.

[107] T. Ishii, S. Tsuboi, G. Sakane, M. Yamashita, B. K. Breedlove, Dalton Trans. 2009, 680687.

[108] D. M. D'Alessandro, F. R. Keene, Chem. Soc. Rev. 2006, 35, 424-440.

[109] D. Sil, S. Dey, A. Kumar, S. Bhowmik, S. P. Rath, Chem. Sci. 2016, 7, 1212-1223.

[110] S. Shin, E. T. Yukl, E. Sehanobish, C. M. Wilmot, V. L. Davidson, Biochemistry 2014, 53, 1342-1349.

[111] J. Geng, K. Dornevil, V. L. Davidson, A. Liu, Proc. Natl. Acad. Sci. U.S.A. 2013, 110, 96399644.

[112] K. E. Brancato-Buentello, S.-J. Kang, W. R. Scheidt, J. Am. Chem. Soc. 1997, 119, 28392846.

[113] A. Takai, C. P. Gros, J.-M. Barbe, R. Guilard, S. Fukuzumi, Chem. Eur. J. 2009, 15, 31103122.

[114] J.-M. Lu, S. V. Rosokha, J. K. Kochi, J. Am. Chem. Soc. 2003, 125, 12161-12171.

[115] K. M. J. Sanders, N. Bampos, Z. Clyde-Watson, S. L. Darling, J. C. Hawley, H.-J. Kim, C. C. Mak, S. J. Webb in The Porphyrin Handbook, Vol. 3; (Eds. K. M. Kadish, K. M. Smith, R. Guilard), Academic Press, San Diego, 2000, pp. 1-48.

[116] S. Wang, Z. Li, X. Wang, X. Yu, Acta Crystallogr, Sect. E: Struct. Rep. Online 2008, 64, m1232.

[117] C. Yang, M. Y. Tsai, S.-W. Hung, J.-H. Chen, S.-S. Wang, J.-Y. Tung, Polyhedron 2012, 37, $1-8$.

[118] J. F. Kirner, C. A. Reed, W. R. Scheidt, J. Am. Chem. Soc. 1977, 99, 2557-2563.

[119] E. Bill, julX: Magnetic Simulation; Max-Planck-Institute for Chemical Energy Conversion, Mülheim/Ruhr, Germany.

[120] O. Kahn, Molecular magnetism; Wiley-VCH, New York, NY, 1993.

[121] L. Penkova, S. Demeshko, V. A. Pavlenko, S. Dechert, F. Meyer, I. O. Fritsky, Inorg. Chim. Acta 2010, 363, 3036-3040.

[122] L. Stoicescu, A. Jeanson, C. Duhayon, A. Tesouro-Vallina, A. K. Boudalis, J.-P. Costes, J.P. Tuchagues, Inorg. Chem. 2007, 46, 6902-6910.

[123] M. Viciano-Chumillas, S. Tanase, I. Mutikainen, U. Turpeinen, de Jongh, L Jos, J. Reedijk, Dalton Trans. 2009, 7445-7453.

[124] A. Das, S. Demeshko, S. Dechert, F. Meyer, Eur. J. Inorg. Chem. 2011, 1240-1248.

[125] D. P. Goldberg, J. Telser, J. Krzystek, A. G. Montalban, L.-C. Brunel, Barrett, Anthony G. M, B. M. Hoffman, J. Am. Chem. Soc. 1997, 119, 8722-8723.

[126] G. N. Newton, T. Shiga, H. Oshio, Sci. China Chem. 2012, 55, 973-977.

[127] B. J. Kennedy, K. S. Murray, Inorg. Chem. 1985, 24, 1557-1560.

[128] a) D. V. Behere, S. Mitra, Inorg. Chem. 1980, 19, 992-995; b) D. P. Goldberg, A. G. Montalban, A. J. P. White, D. J. Williams, Barrett, Anthony G. M, B. M. Hoffman, Inorg. Chem. 1998, 37, 2873-2879.

[129] K. M. Kadish, E. van Caemelbecke, G. Royal in The Porphyrin Handbook, Vol. 8; (Eds. K. M. Kadish, K. M. Smith, R. Guilard), Academic Press, San Diego, 2000, pp. 1-114.

[130] J. W. Buchler, C. Dreher, K. L. Lay, Z. Naturforsch, B: J. Chem. Sci. 1982, 37B, 1155-1162.

[131] J. Du Bois, J. Hong, E. M. Carreira, M. W. Day, J. Am. Chem. Soc. 1996, 118, 915-916.

[132] F. L. Neely, L. A. Bottomley, Inorg. Chim. Acta 1992, 192, 147-149. 
[133] A. Vogel, S. Dechert, C. Brückner, F. Meyer, Inorg. Chem. 2017; DOI: 10.1021/acs.inorgchem.6b02916.

[134] J. W. Buchler in The Porphyrins, Vol. 1; (Ed. D. Dolphin), Elsevier Science, Oxford, 1978, pp. 389-483.

[135] A. Ghosh, J. Almloef, J. Phys. Chem. 1995, 99, 1073-1075.

[136] R. D. Shannon, Acta Crystallogr, Sect. A: Found. Crystallogr. 1976, 32, 751-767.

[137] D. R. Lide, CRC handbook of chemistry and physics: A ready-reference book of chemical and physical data; CRC Press, Boca Raton, 2003.

[138] E. Bill, ESIM, Mülheim an der Ruhr, 2016.

[139] E. Bill, EVIEW, Mülheim an der Ruhr, 2016.

[140] E. Stephen, A. J. Blake, E. Carter, D. Collison, E. S. Davies, R. Edge, W. Lewis, D. M. Murphy, C. Wilson, R. O. Gould, A. J. Holder, J. McMaster, M. Schröder, Inorg. Chem. 2012, $51,1450-1461$.

[141] W.-P. To, Y. Liu, T.-C. Lau, C.-M. Che, Chem. Eur. J. 2013, 19, 5654-5664.

[142] a) N. G. Connelly, W. E. Geiger, Chem. Rev. 1996, 96, 877-910; b) I. Noviandri, K. N. Brown, D. S. Fleming, P. T. Gulyas, P. A. Lay, A. F. Masters, L. Phillips, J. Phys. Chem. B 1999, 103, 6713-6722.

[143] G. M. Sheldrick, Acta Crystallogr, Sect. A: Found. Crystallogr. 2008, 64, 112-122.

[144] H. G. O. Becker, R. Beckert, Organikum: Organisch-chemisches Grundpraktikum; WileyVCH, Weinheim, 2004.

[145] a) J. L. Sessler, A. Mozaffari, M. R. Johnson, Org. Synth. 1991, 70, 68-77; b) G. D. Hartman, L. M. Weinstock, L. E. Benjamin, N. W. Gilman, G. Saucy, Org. Synth. 1988, 6, 620.

[146] a) F. Neese, ORCA: An ab initio, DFT and semiempirical SCF-MO package, Max-PlanckInstitute for Chemical Energy Conversion, Mülheim an der Ruhr, Germany, 2013; b) F. Neese, Wiley Interdiscip. Rev.: Comput. Mol. Sci. 2012, 2, 73-78.

[147] a) A. D. Becke, Phys. Rev. A 1988, 38, 3098-3100; b) J. P. Perdew, Phys. Rev. B 1986, 33, 8822-8824.

[148] A. Schäfer, H. Horn, R. Ahlrichs, J. Chem. Phys. 1992, 97, 2571-2577.

[149] F. Weigend, R. Ahlrichs, Phys. Chem. Chem. Phys. 2005, 7, 3297-3305.

[150] A. D. Becke, J. Chem. Phys. 1993, 98, 1372-1377.

[151] F. Weigend, Phys. Chem. Chem. Phys. 2006, 8, 1057-1065.

[152] a) S. Kossmann, F. Neese, J. Chem. Theory Comput. 2010, 6, 2325-2338; b) D. Andrae, U. Häußermann, M. Dolg, H. Stoll, H. Preuß, Theoret. Chim. Acta 1990, 77, 123-141. 


\section{INDEX}

\begin{tabular}{|c|c|c|c|}
\hline aromaticity & 4 & HEISENBERG-DIRAC-VAN VLECK & Hamiltonian \\
\hline HÜCKEL & 5 & & 53 \\
\hline MöBIUS & 5 & helical pitch & 24 \\
\hline BILL & 52 & hemoglobin & 1 \\
\hline BLUSCH & 10 & hexaphyrin & 6 \\
\hline BROOKER & 37 & HILL & 2 \\
\hline CHANG & 2 & HOMO-LUMO gap & 77 \\
\hline charge resonance & 47 & IR spectroscopy & \\
\hline chlorophyll & 1 & $27 \mathbf{M n}_{2}{ }^{a z}$ & 56 \\
\hline Claisen & 38 & JAHN-TELLER & 50 \\
\hline cobalamin & 1 & LEWIS & 38 \\
\hline cyclic voltammetry & & LIND & 10 \\
\hline $27 \mathrm{H}_{2} \mathrm{Pd}$ & 70 & luminescence & 60 \\
\hline $27 \mathrm{Mn}_{2}{ }^{\mathrm{ac}}$ & 55 & magnetic susceptibility & \\
\hline $27 \mathrm{Mn}_{2}{ }^{\mathrm{az}}$ & 55 & $27 \mathbf{M n}_{2}{ }^{\text {ac }}$ & 52 \\
\hline cytochrome P450 & 1 & $27 \mathrm{Mn}_{2}{ }^{\mathrm{az}}$ & 52 \\
\hline DDQ & 17 & 27NiMnac & 54 \\
\hline derivatization & 36 & mass spectrometry & \\
\hline DFT calculations & & $27 \mathrm{H}_{2} \mathrm{Pd}$ & 59 \\
\hline $27 \mathbf{H}_{2} \mathbf{P d}^{+}$ & 79 & $27 \mathrm{Mn}_{2}{ }^{\mathrm{ac}}$ & 43 \\
\hline $27 \mathrm{H}_{2} \mathbf{P d}^{-}$ & 79 & $27 \mathrm{Mn}_{2}{ }^{\mathrm{az}}$ & 43 \\
\hline dipyrrin & 24 & 27NiMnac & 45 \\
\hline EPR spectroscopy & & $27 \mathbf{P d}_{2}$ & 59 \\
\hline $27 \mathrm{H}_{2} \mathrm{Pd}^{+}$ & 75 & $32 \mathrm{H}_{3}$ & 18 \\
\hline $27 \mathrm{H}_{2} \mathrm{Pd}^{-}$ & 75 & $33 \mathrm{H}_{2}$ & 19 \\
\hline FISCHER & 17 & $34 \mathrm{Ni}$ & 32 \\
\hline FRENSCH & see BluSCH & $35 \mathrm{HNi}$ & 33 \\
\hline GROVES & 2 & MEUNIER & 2 \\
\hline \multirow[t]{2}{*}{ HAMMETT } & 12 & MITEVSKI & 12 \\
\hline & & myoglobin & 1 \\
\hline
\end{tabular}




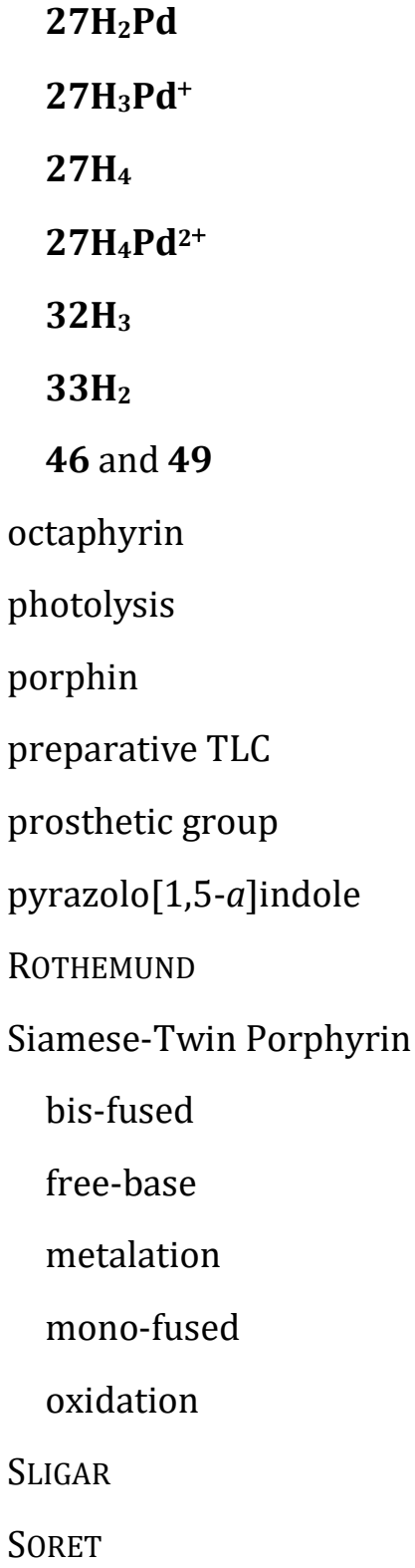

67

67

27

67

27

27

39

8

56

1

17

1

24

17

19

10

$12,42,58$

17

17

2

19

spectroelectrochemistry

27 $\mathrm{H}_{2} \mathrm{Pd}$

SQUID

tautomerism

73

52

62,79

\section{UV/vis spectroscopy}

$$
\text { 27 } \mathrm{H}_{2} \mathbf{P d}
$$

60

$\mathbf{2 7} \mathbf{H}_{3} \mathbf{P d}^{+}$

61

$27 \mathrm{H}_{4} \mathbf{P d}^{++}$

61

$27 \mathrm{H}_{6}{ }^{2+}$

12

$27 \mathrm{Mn}^{\text {ac }}$

45

$27 \mathrm{Mn}_{2}{ }^{\mathrm{az}}$

45

20

20

33

33

21

62

21

48

51,52

52

64

24

26

33

39

46 and 49

53

ZEEMAN

54

zero field splitting

21

$\beta$-ethylporphyrins

27 


\section{LIST OF SCIENTIFIC CONTRIBUTIONS}

\section{POSTERS}

JungChemiker Tagung, Münster (Germany)

Apr 2015

The Siamese-Twin Porphyrin - A Story with a Twist

JungChemiker Tagung, Göttingen (Germany)

Jun 2015

Oxidation of the Siamese-Twin Porphyrin: A Story with a Twist

$6^{\text {th }}$ EUCheMS Conference on Nitrogen Ligands, Beaune (France)

Sep 2015

Manganese Complexes of the Siamese-Twin Porphyrin

$9^{\text {th }}$ International Conference on Porphyrins and Phthalocyanines, Nanjing (China) Jul 2016

On the Twists and Turns of the Siamese-Twin Porphyrin upon Oxidation

\section{Presentations}

JungChemiker Tagung, Göttingen (Germany)

Jun 2015

Manganese Complexes of the Siamese-Twin Porphyrin 



\section{CURRICULUM VITAE}

\section{Personal Data}

Name

Date of birth

Place of birth

Nationality
Anastasia Vogel

June 9, 1987

Ekibastus, Kazakhstan

German

\section{ACADEMIC CAREER}

Georg-August-University Göttingen, Institute of Inorganic Chemistry

Jan 2013 - Oct 2016

PhD candidate, Advisor: Prof. Dr. Franc Meyer

Thesis: The Siamese Twin-Porphyrin: A Bimetallic Platform for Multi-Electron Redox Chemistry

\section{Georg-August-University Göttingen}

Oct 2010 - Nov 2012

M.Sc. in Chemistry

Focus: Synthetic Inorganic Chemistry

Thesis: New Complexes of the Siamase-Twin Porphyrin

Georg-August-University Göttingen

Oct 2007 - Sep 2010

B.Sc. in Chemistry

Focus: Synthetic Inorganic Chemistry

Thesis: Methanobactin als Vorbild: Neue Liganden für die Cu(I)-Komplexierung

\section{INTERNATIONAL EXPERIENCE}

University of Connecticut, Storrs, USA

Jan 2014 - Apr 2014

Visiting researcher, group of Prof. Dr. Christian Brückner

\section{TEACHING EXPERIENCE}

Method course "Methoden der Chemie II"

Teaching assistant, annual exercise course (UV/vis, Mößbauer, SQUID)

Method course "Methoden der anorganischen Chemie"

Teaching assistant, semiannual practical course (UV/vis, EPR, cyclic voltammetry)

Exercise course "Stuktur und Bindung II"

Oct 2012 - Mar 2013

Teaching assistant, advanced exercise course (coordination chemistry)

Synthetic course "Fortgeschrittenes Synthesepraktikum"

Oct 2012 - Mar 2013

Teaching assistant, advanced practical course (complex synthesis) 


\section{HONORS AND AWARDS}

Georg-August-University, scholarship

Jan 2016

Studienstiftung des Deutschen Volkes, scholarship

Sep 2013

Georg-August-University, starter's scholarship

Jan 2013

Studienstiftung des Deutschen Volkes, scholarship

Jan 2008

e-fellows.net, scholarship

Jun 2007

GDCh-award for top of the class in chemistry

Jun 2007

Graduated top of the class

Jun 2007

\section{Publications}

A. C. Jahnke, A. Herter, S. Dechert, M. John, F. Meyer, Inorg. Chim. Acta 2011, 374, 601-605.

A. Vogel, S. Dechert, M. John, C. Brückner, F. Meyer, Chem. Eur. J. 2016, 22, 2307-2316.

A. Vogel, S. Dechert, C. Bruckner, F. Meyer, Inorg. Chem. 2017; DOI: 10.1021/acs.inorgchem.6b02916. 